CONTROL OF SPACECRAFT FORMATION FLYING AROUND ASTEROIDS

\author{
by \\ Arthur Kar Leung LIN \\ B. Eng., Aerospace Engineering, Ryerson University, 2007 \\ M.A.Sc., Aerospace Engineering, Ryerson University, 2009
}

\author{
A dissertation \\ presented to Ryerson University \\ in partial fulfillment of the \\ requirements for the degree of \\ DOCTOR OF PHILOSOPHY \\ in the Program of \\ Aerospace Engineering
}

Toronto, Ontario, Canada, 2014

(C)Arthur Kar Leung LIN 2014

All rights reserved 


\section{Author's Declaration}

I hereby declare that I am the sole author of this dissertation. This is a true copy of the dissertation, including any required final revisions, as accepted by my examiners.

I authorize Ryerson University to lend this dissertation to other institutions or individuals for the purpose of scholarly research.

I further authorize Ryerson University to reproduce this dissertation by photocopying or by other means, in total or in part, at the request of other institutions or individuals for the purpose of scholarly research.

I understand that my dissertation may be made electronically available to the public. 


\title{
Abstract \\ CONTROL OF SPACECRAFT FORMATION FLYING AROUND ASTEROIDS
}

\author{
Arthur Kar Leung Lin, Doctor of Philosophy, Aerospace Engineering \\ Ryerson University, Toronto, June 2014
}

There exist thousands of different minerals and other possible resources out in space. To exploit these resources and to further expand our knowledge of the universe, planetary exploration has opened new gates towards mankind. There are more than one hundred thousand designated asteroids located inside the asteroid belt. Some of these asteroids are as old as the Big Bang itself. Tracking of astronomical bodies such as asteroids is the new stream of research that has attracted a lot of attention. However, due to environmental constraints around asteroids, monolithic spacecraft missions seem challenging. Multi-agent systems, on the other hand, provide significant advantages when it comes to orbiting around asteroids. In this study, novel consensus algorithms are applied to regulate the multi-agent decentralized formation flying for increased system flexibility and reliability. A nonlinear controller is developed to control the decentralized formation flying system of interest.

Faults are evaluated and reduced to a minimum when planning a mission. However, the performance of the controller should not be affected when faults occur. For this reason, sensor and actuator faults are examined in this thesis in conjunction with actuator limitations which is commonly referred to as saturation. The proposed control law is not only able to control the system while faults occur, but rather it is capable of maintaining system stability in the presence of time variant external disturbances. Uncertainty in parameters and dynamic models are inevitable due to the complexity of the relatively new mission and lack of experimental data about the system dynamics. As such, a novel adaptive robust control methodology is developed that does not require full knowledge of the system dynamics. Moreover, the adaptive robust control law is combined with a Chebyshev neural network to overcome system uncertainties. Numerical simulations results along with stability analyses show that the proposed control methodology is capable of reducing the system state error close to zero within 1 orbit when maximum thrust of $5 \mathrm{mN}$ with bounded external disturbance of $3 \mathrm{mN}$ is applied for formation reconfiguration scenarios; these results will be useful for the future formation flying missions around asteroids. 


\section{Acknowledgements}

I would like to express my sincere gratitude to my supervisor, Professor Krishna Dev Kumar, for his timely guidance, encouragement and support. 5 years ago, I was a rookie in space system dynamics and control discipline. If not because of Dr. Kumar, I would not be able to work on such an innovative topic and complete this wonderful dissertation. Thank you for helping me getting through all these challenging "rocket science" problems, and for your guidance and counsels that helped me make significant contributions in the field of Spacecraft Dynamics and Control. Your judicious suggestions made the successful completion of this thesis possible. I would like to extend this appreciation to Professor Puren Ouyang, Professor Anton de Ruiter and Professor Simant R. Upreti for their invaluable comments as members of my dissertation committee.

I am grateful to my colleagues and friends with whom I had the greatest time. Names are not provided because they know who they are. Exclusive thanks to Siavash Khajehhasani and Sobhan Etemadi. They have been the best possible mentor in every sense of the word. Without that time table you, Siavash, set up for me, I would not be able to complete my thesis. Even though, I don't have my 6 pack yet, I can do hand stand, cart wheel, pull up, 90 lbs bench press, all and all that I can almost have another PhD degree in gymnastics. Sobhan, you make me a stronger man literally. I give my special appreciation to Janaya Denys for the careful proof-reading of my thesis. She made this thesis much more readable.

I would like to take this opportunity to thank my parents Raymond Chun-Lau Lin, and Lucenla Lai-King Lin Wong who deserve special recognition for their continuous encouragement and selfless support. In the path to this success, I have been fallen thousands of time, if not because of my parents, I won't have the strength and perseverance to reach my current status. 
$\mathscr{\%}$

My Quaing Parents, Pister, Friends and Framily 


\section{Table of Contents}

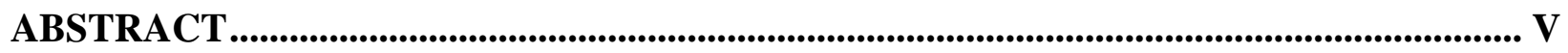

ACKNOWLEDGEMENTS ............................................................................................... VII

TABLE OF CONTENTS .........................................................................................................

LIST OF FIGURES ................................................................................................................

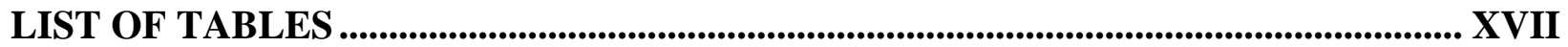

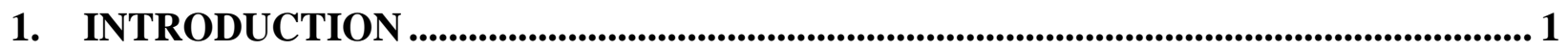

1.1. RESEARCH MOTIVATION .................................................................................... 1

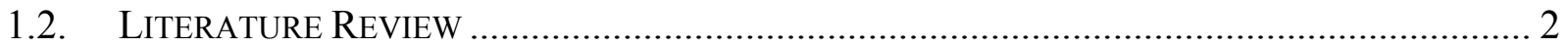

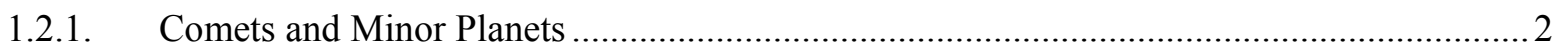

1.2.2. Spacecraft Formation Flying Missions ……………………………………………..... 4

1.2.3. Consensus Spacecraft Formation Control ......................................................................... 6

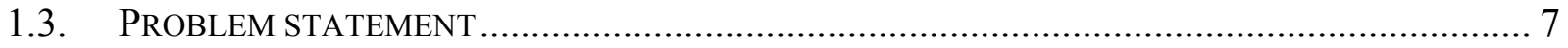

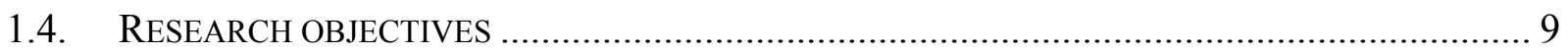

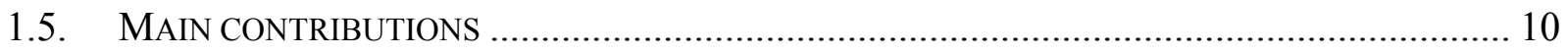

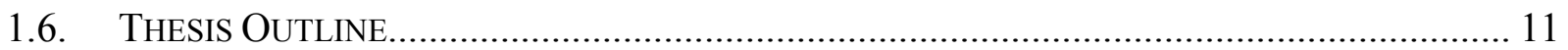

2. SYSTEM MODEL AND MULTI-AGENT SYSTEM ..................................................... 13

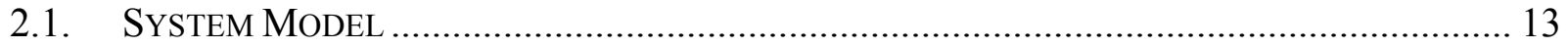

2.1.1. Spacecraft Orbital Dynamics Around an Asteroid.......................................................... 14

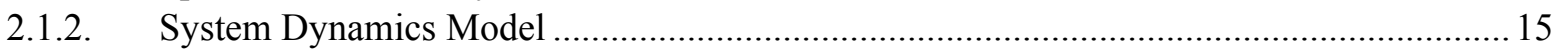

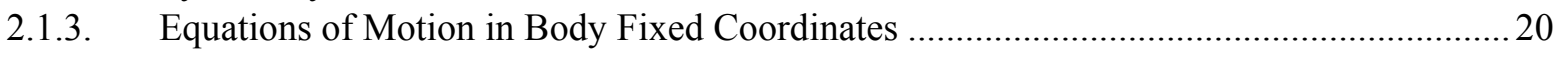

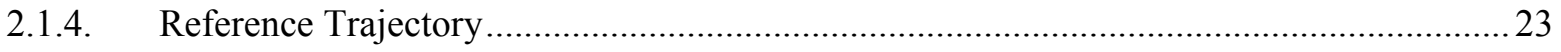

2.1.5. External disturbances ...................................................................................... 24

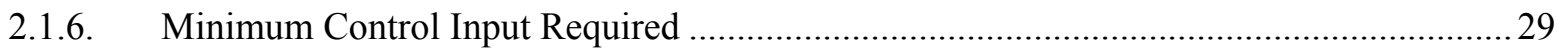

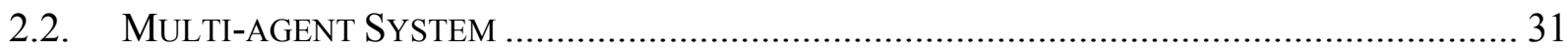

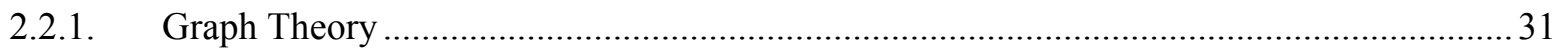

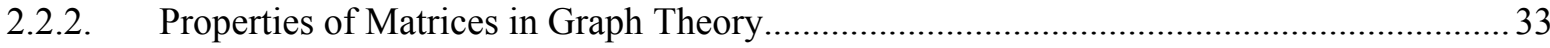

2.2.3. Consensus Algorithm.......................................................................................... 35

2.2.4. Decentralized Formation Flying Mathematical Model ...................................................... 35

3. SLIDING MODE CONTROL OF CONSENSUS SPACECRAFT FORMATION

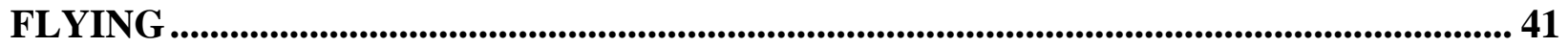

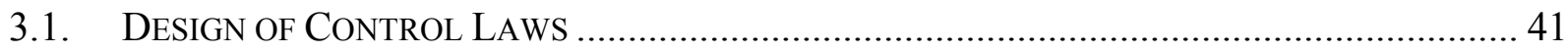

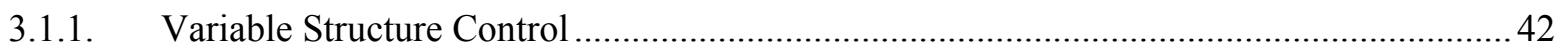

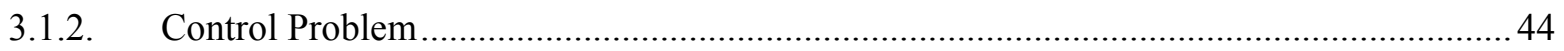

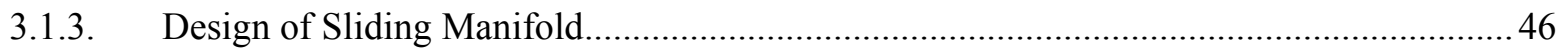

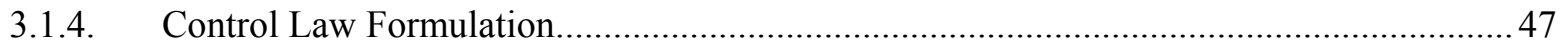

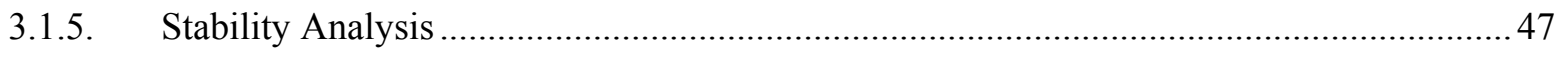

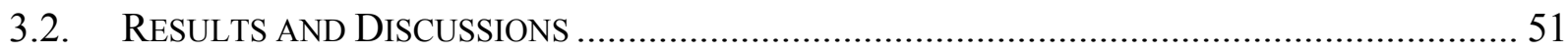




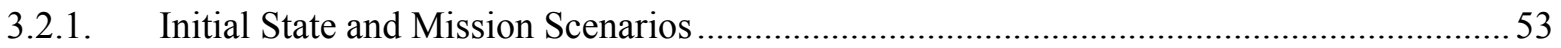

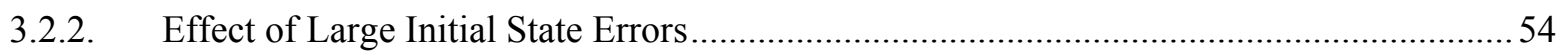

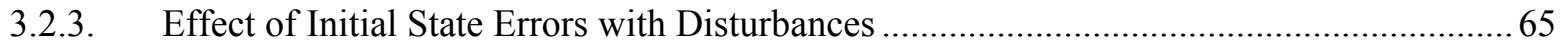

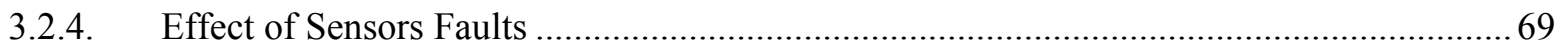

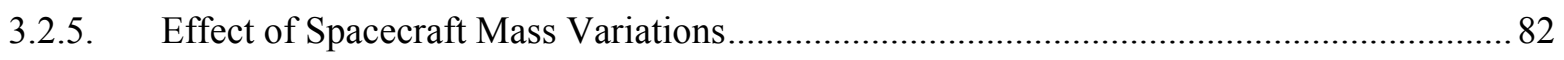

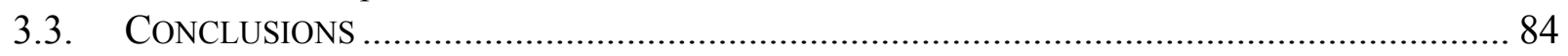

4. ROBUST CONTROL OF CONSENSUS SPACECRAFT FORMATION FLYING ... 85

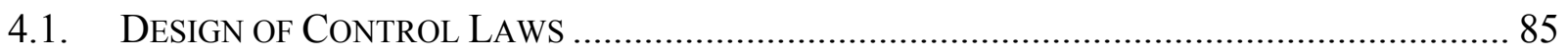

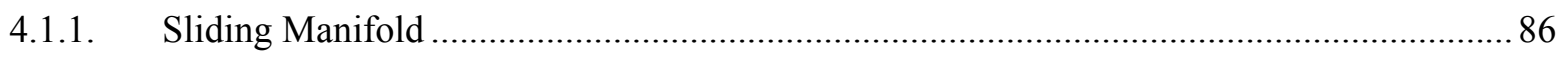

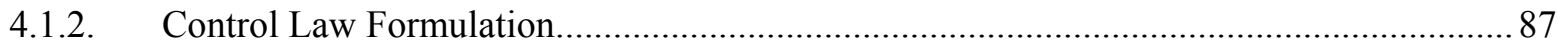

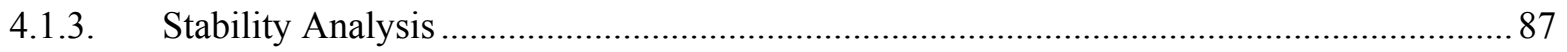

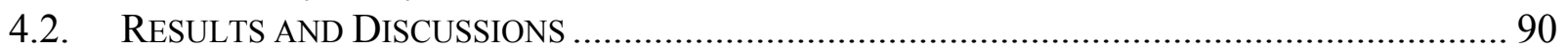

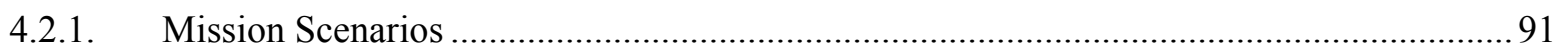

4.2.2. Effect of Large Initial State Errors with Disturbances..................................................... 92

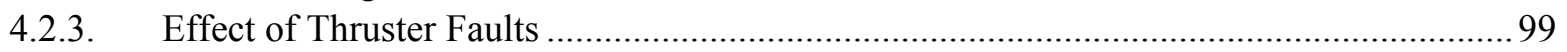

4.2.4. Effect of Multiple Actuator Faults for Different Asteroids............................................ 103

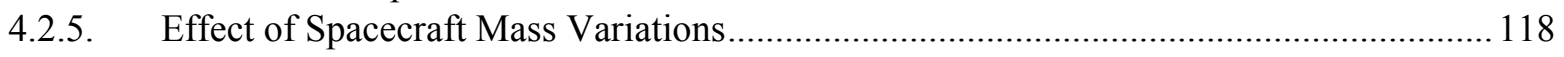

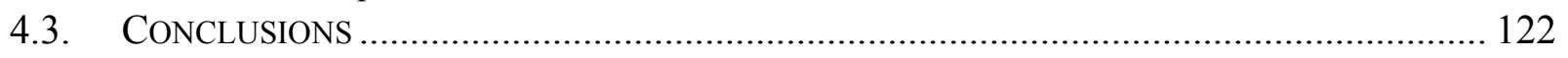

\section{NEURAL NETWORK ADAPTIVE CONTROL OF CONSENSUS SPACECRAFT}

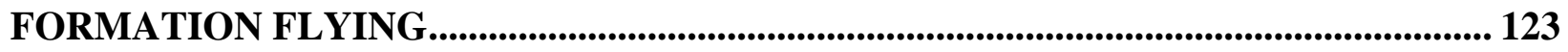

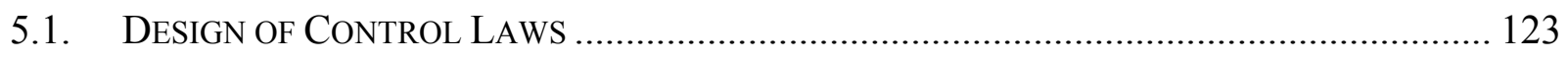

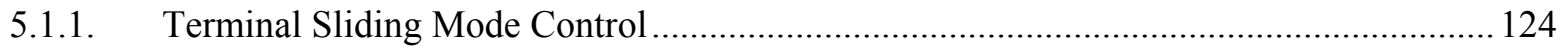

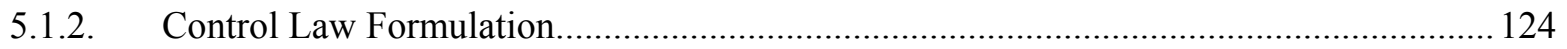

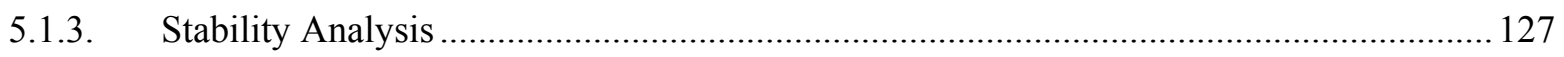

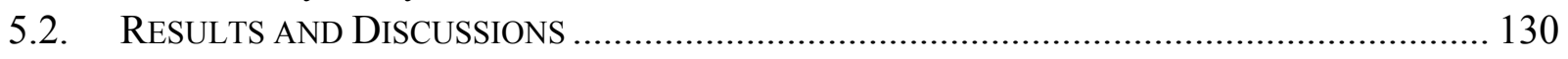

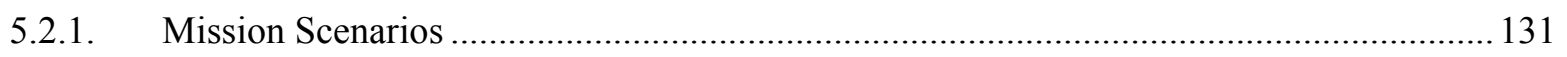

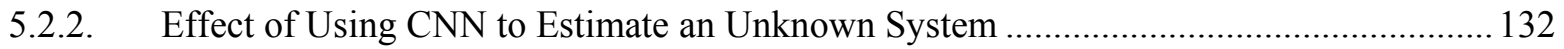

5.2.3. Performance Comparison with SMC and Robust Control ............................................. 142

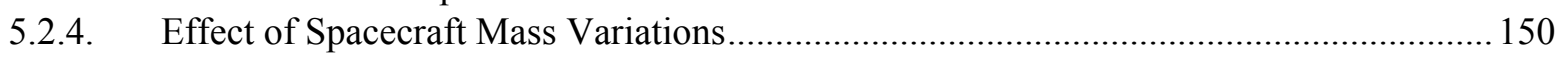

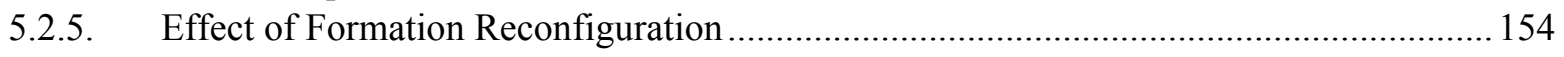

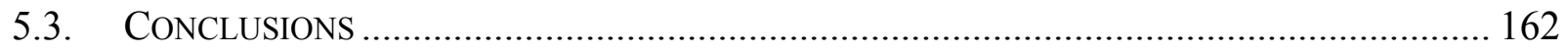

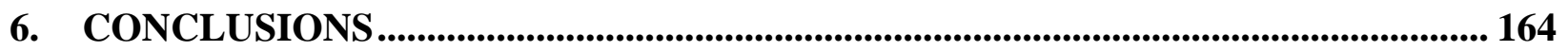

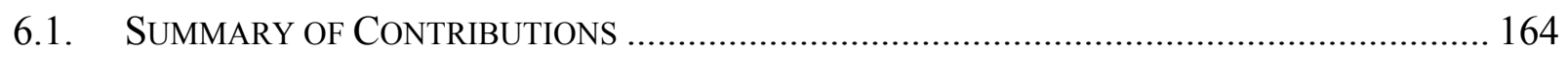

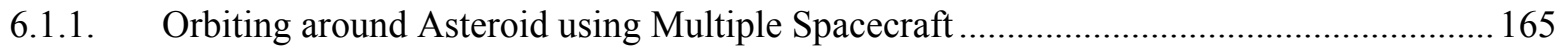

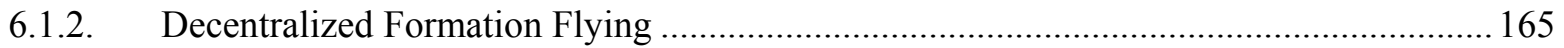

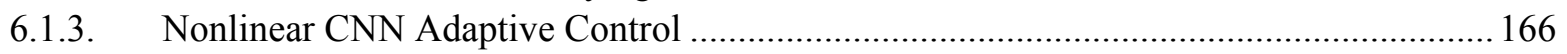

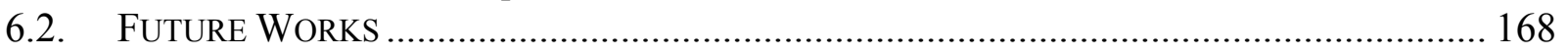

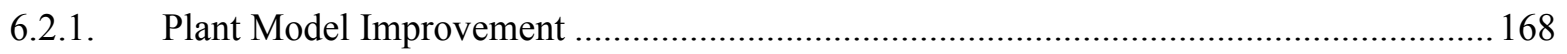

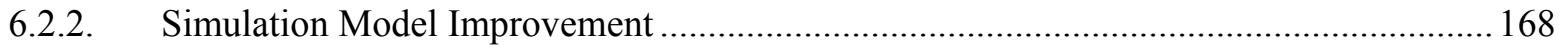

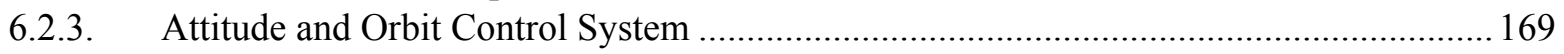

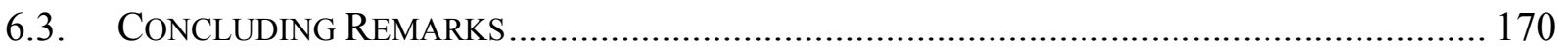

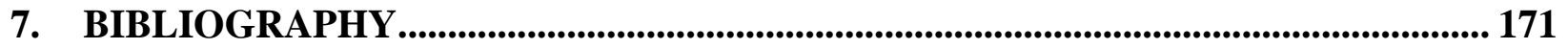




\section{List of Figures}

FIGURE 2.1 POSITION GEOMETRY OF A POINT MASS OF AN ASTEROID IN THE BODY FIXED SPACE [8] ......... 15

FIGURE 2.2 GEOCENTRIC EQUATORIAL FRAME AND THE ORBITAL ELEMENTS [63] .................................. 19

FIGURE 2.3 GEOMETRY OF THE INERTIAL AND BODY-FIXED COORDINATES...........................................22

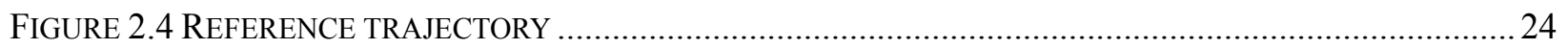

FIGURE 2.5 RELATIVE POSITION VECTORS IN THE SUN-ASTEROID-SPACECRAFT SYSTEM.........................26

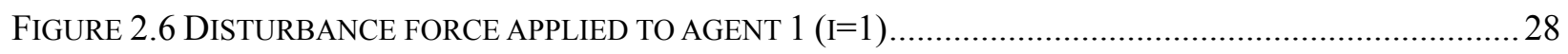

FIGURE 2.7 MINIMUM CONTROL INPUT REQUIRED (WITHOUT DISTURBANCE) ....................................... 30

FIGURE 2.8 MINIMUM CONTROL INPUT REQUIRED FOR AGENT 1 (WITH DISTURBANCE) ........................... 30

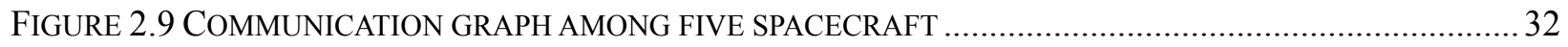

FIGURE 2.10 (A) STRONGLY CONNECTED UNDIRECTED GRAPH, (B) ROOTED DIRECTED TREE .....................33

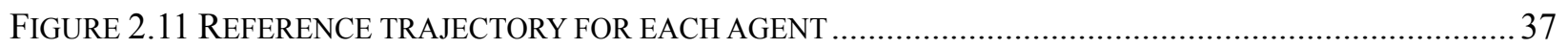

FIGURE 3.1: PHASE PORTRAIT OF THE DOUBLE INTEGRATOR UNDER VSC …..........................................43

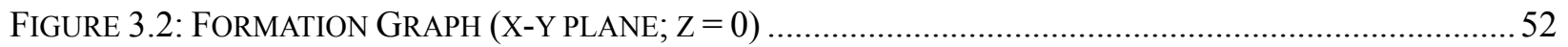

FIGURE 3.3: SYSTEM STATE SNAP SHOT AT DIFFERENT TIME (WITHOUT THRUSTER SATURATION) ...............55

FIGURE 3.4: SYSTEM STATE SNAP SHOT AT DIFFERENT TIME (WITH 10 MN THRUSTER SATURATION) .......... 55

FIGURE 3.5: SYSTEM STATE SNAP SHOT AT DIFFERENT TIME (WITH 5 MN THRUSTER SATURATION) ............ 56

FIGURE 3.6: RELATIVE POSITION ERROR (WITHOUT THRUSTER SATURATION) ..........................................57

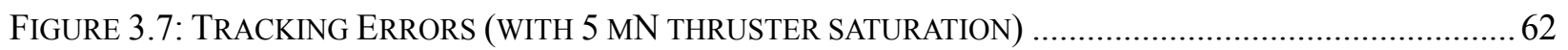

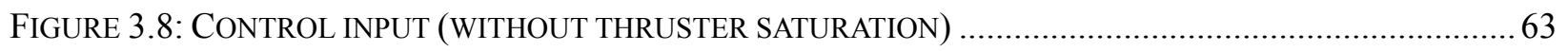

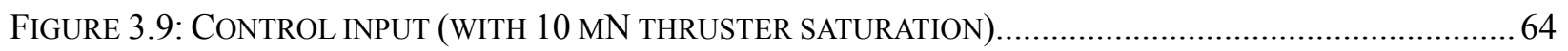

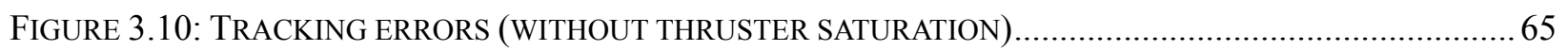

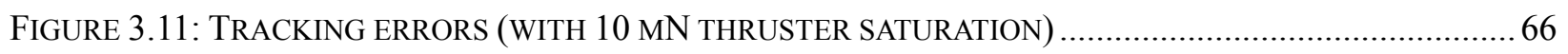

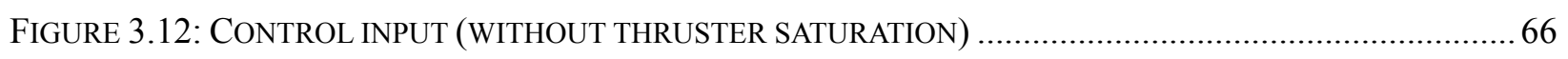

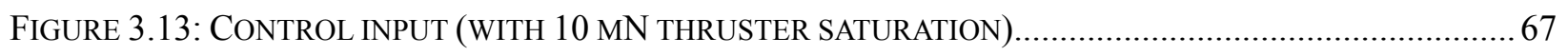

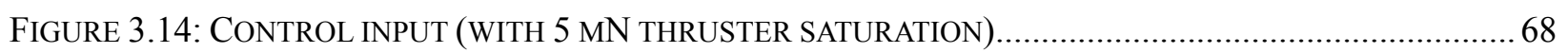

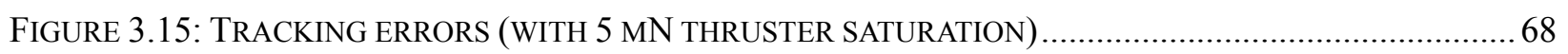

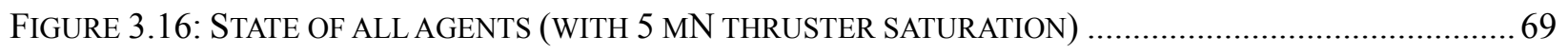

FIGURE 3.17: SYSTEM STATE SNAP SHOT AT DIFFERENT TIME (WITHOUT THRUSTER SATURATION) ............. 70

FIGURE 3.18: TWO 3D VIEWS OF SYSTEM STATE (WITHOUT THRUSTER SATURATION) ............................... 70

FIGURE 3.19: STATE OF ALL AGENTS (WITHOUT THRUSTER SATURATION) ….......................................... 71

FIGURE 3.20: TRACKING ERRORS (WITHOUT THRUSTER SATURATION) ….............................................. 72 
FIGURE 3.21: CONTROL INPUT FOR ALL AGENTS (WITHOUT THRUSTER SATURATION) ............................... 72

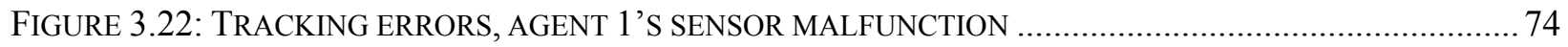

FIGURE 3.23: TRACKING ERRORS, AGENT 1, 2 AND 3'S SENSOR MALFUNCTION ….................................. 74

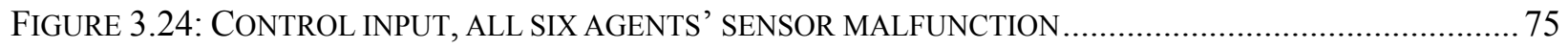

FIGURE 3.25: CONTROL INPUT, ALL SIX AGENTS' SENSOR MALFUNCTION (WITH 5 MN SATURATION) ........ 76

FIGURE 3.26: TRACKING ERRORS, ALL AGENTS' SENSOR MALFUNCTION (WITH 5 MN SATURATION) .......... 76

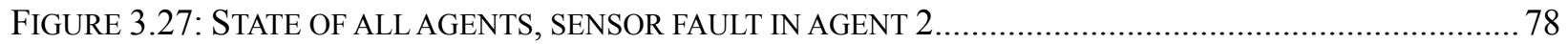

FIGURE 3.28: STATE OF ALL AGENTS, SENSOR FAULT IN AGENT 2, 4 AND 6 .............................................. 78

FIGURE 3.29: CONTROL INPUT, AGENT 2, 4 AND 6' SENSOR MALFUNCTION (WITH 5 MN SATURATION) ..... 79

FIGURE 3.30: CONTROL INPUT, ALL SIX AGENTS' SENSOR MALFUNCTION (WITH 5 MN SATURATION) ........ 80

FIGURE 3.31: TRACKING ERRORS, AGENT 2, 4, AND 6' SENSOR MALFUNCTION (WITH 5 MN SATURATION) 81

FIGURE 3.32: TRACKING ERRORS, ALL AGENTS' SENSOR MALFUNCTION (WITH 5 MN SATURATION) .......... 81

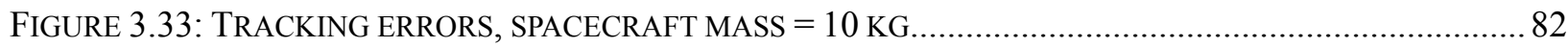

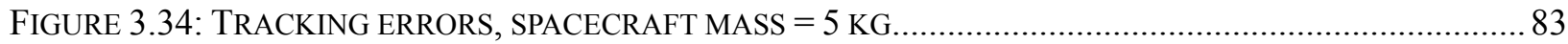

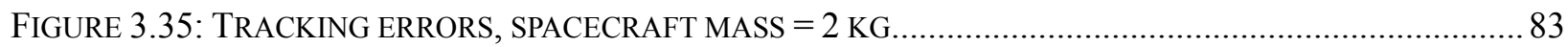

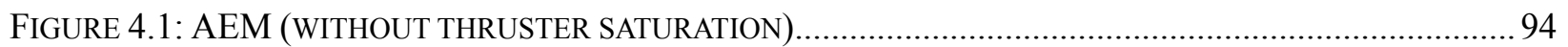

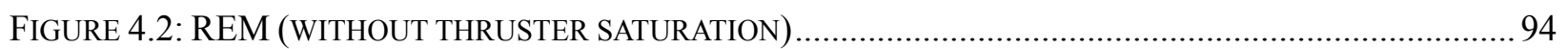

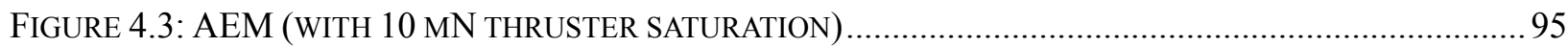

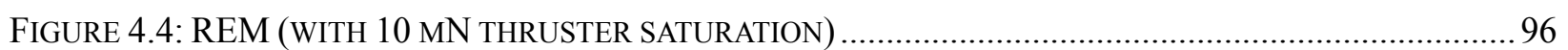

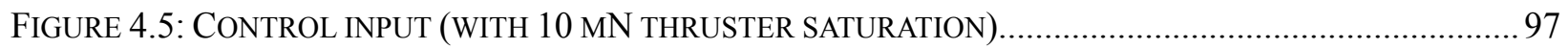

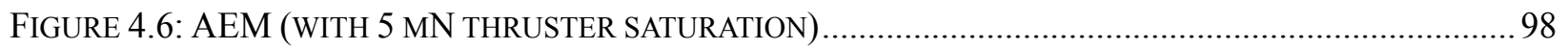

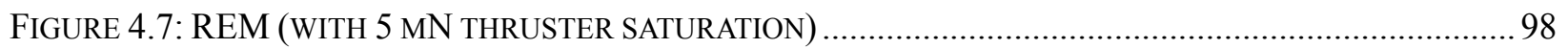

FIGURE 4.8: TRACKING ERRORS, AGENT 6'S THRUSTER MALFUNCTION .................................................. 100

FIGURE 4.9: TRACKING ERRORS, AGENT 4, 5, AND 6'S THRUSTER MALFUNCTION................................... 101

FIGURE 4.10: TRACKING ERRORS, ALL 6 AGENTS’ THRUSTER MALFUNCTION ....................................... 101

FIGURE 4.11: TRACKING ERRORS, ALL 6 AGENTS' THRUSTER MALFUNCTION (WITH 5 MN THRUSTER

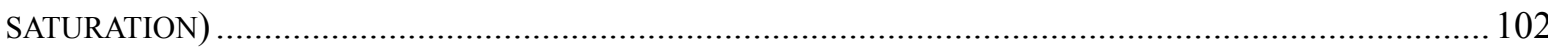

FIGURE 4.12: CONTROL INPUT, ALL SIX AGENTS' THRUSTERS MALFUNCTION (WITH 5 MN SATURATION) 103

FIGURE 4.13: CONTROL INPUT, ALL AGENTS’ THRUSTER MALFUNCTION................................................ 105

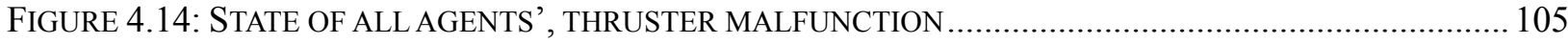

FIGURE 4.15: TRACKING ERRORS, ALL AGENTS’ THRUSTER MALFUNCTION …...................................... 106

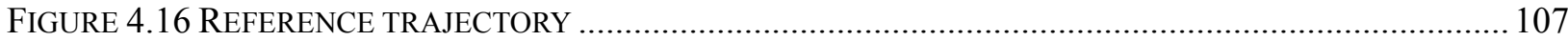

FIGURE 4.17 MINIMUM CONTROL INPUT REQUIRED (WITHOUT DISTURBANCE) .................................... 107

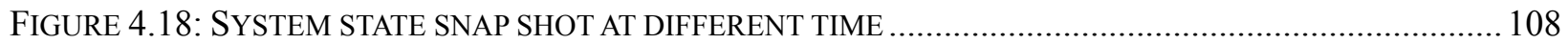




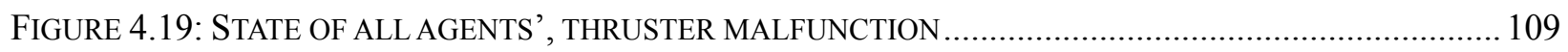

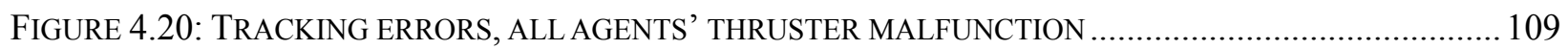

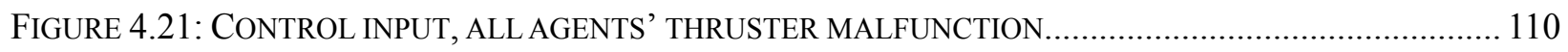

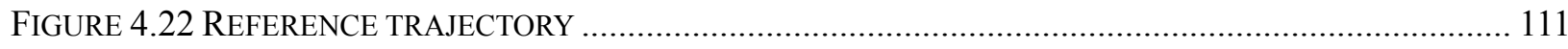

FIGURE 4.23 MINIMUM CONTROL INPUT REQUIRED (WITHOUT DISTURBANCE) …................................ 111

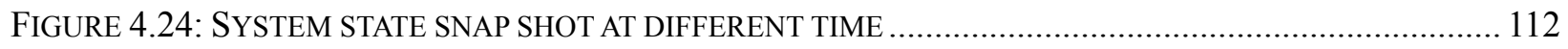

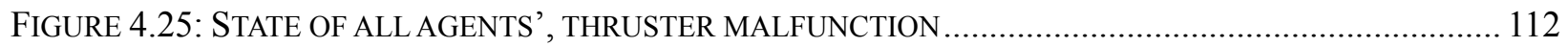

FIGURE 4.26: TRACKING ERRORS, ALL AGENTS’ THRUSTER MALFUNCTION ............................................ 113

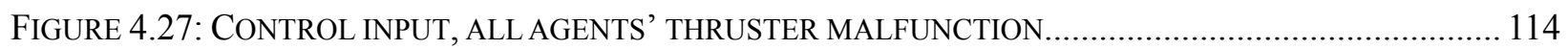

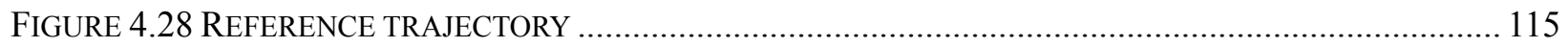

FIGURE 4.29 MINIMUM CONTROL INPUT REQUIRED (WITHOUT DISTURBANCE) ..................................... 115

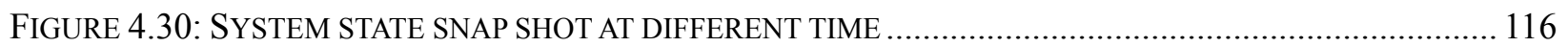

FIGURE 4.31: STATE OF ALL AGENTS', THRUSTER MALFUNCTION ........................................................... 116

FIGURE 4.32: TRACKING ERRORS, ALL AGENTS’ THRUSTER MALFUNCTION ........................................... 117

FIGURE 4.33: CONTROL INPUT, ALL AGENTS’ THRUSTER MALFUNCTION................................................ 117

FIGURE 4.34: TRACKING ERRORS, SPACECRAFT MASS = 2 KG............................................................. 119

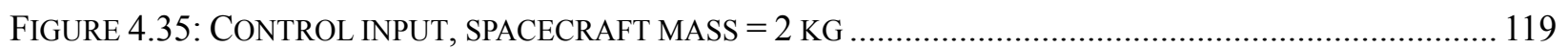

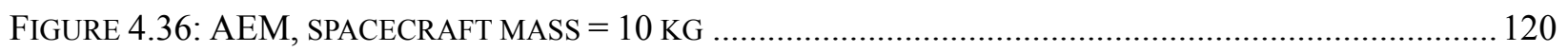

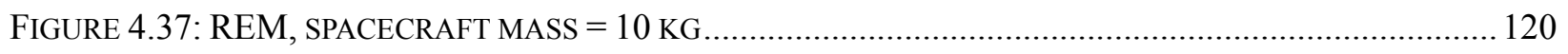

FIGURE 4.38: AEM, SPACECRAFT MASS REDUCE FROM 10 KG TO 1 KG ................................................. 121

FIGURE 4.39: REM, SPACECRAFT MASS REDUCE FROM 10 KG TO 1 KG ............................................... 121

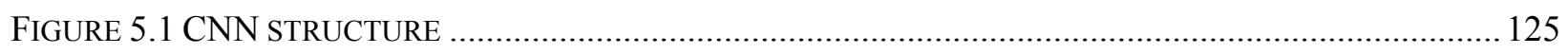

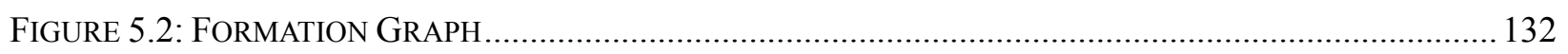

FiguRE 5.3: ESTIMATION BY CNN AND $\boldsymbol{f} \boldsymbol{x}, \boldsymbol{y}, \boldsymbol{z}=\boldsymbol{F}+\boldsymbol{\theta}-\boldsymbol{X} \boldsymbol{d}$ (WITHOUT DISTURBANCE) ................... 133

FiguRE 5.4: ESTIMATION BY CNN AND $\boldsymbol{f} \boldsymbol{x}, \boldsymbol{y}, \boldsymbol{z}=\boldsymbol{F}+\boldsymbol{\theta}-\boldsymbol{X} \boldsymbol{d}$ (WITH DISTURBANCE) ........................ 134

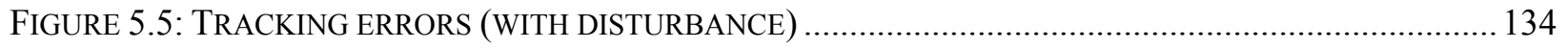

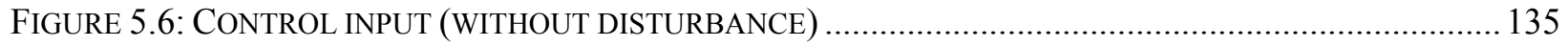

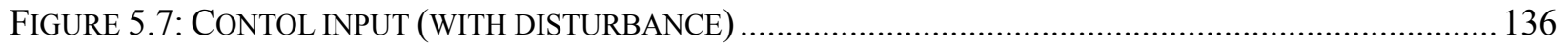

FIGURE 5.8: SYSTEM STATE SNAP SHOT AT DIFFERENT TIME, CENTRALIZED SYSTEM ….......................... 136

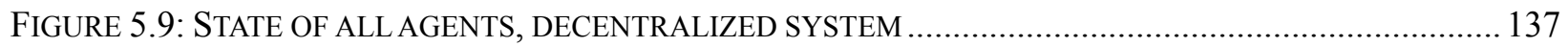

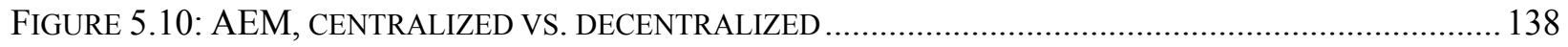

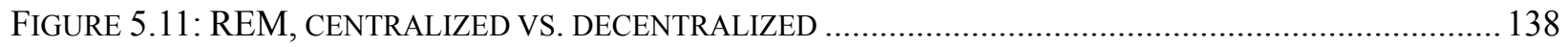

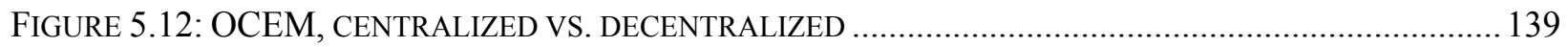

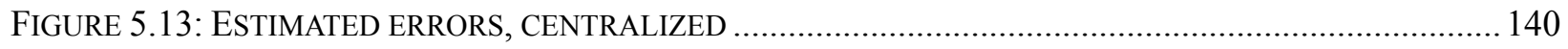




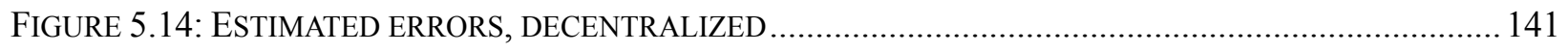

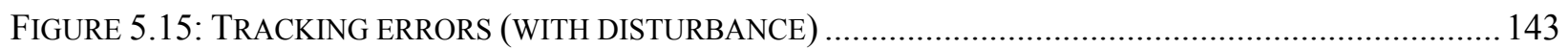

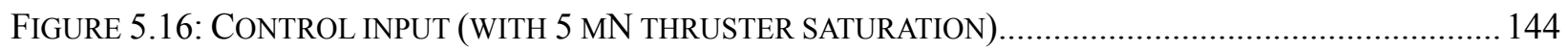

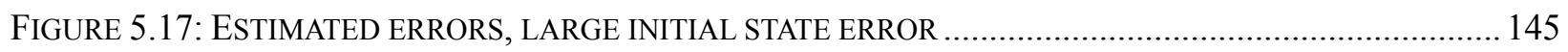

FIGURE 5.18: STATE OF ALL AGENTS, MULTIPLE FAULTS (WITHOUT DISTURBANCE) ................................ 146

FIGURE 5.19: TRACKING ERRORS, MULTIPLE FAULTS (WITH DISTURBANCE) …................................... 147

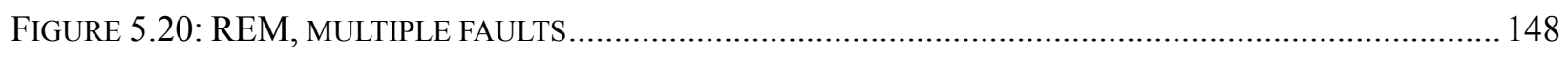

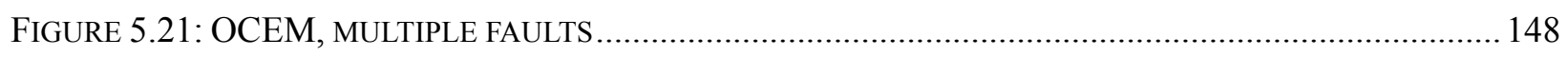

FIGURE 5.22: ESTIMATED ERRORS, MULTIPLE FAULTS (WITHOUT DISTURBANCE) .................................. 149

FIGURE 5.23: ESTIMATED ERRORS, SPACECRAFT MASS = 20 KG ........................................................ 151

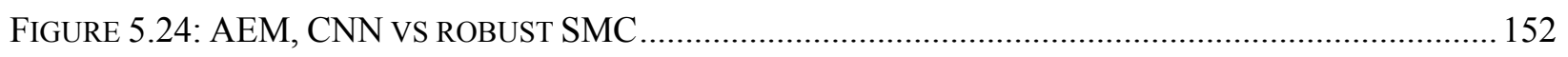

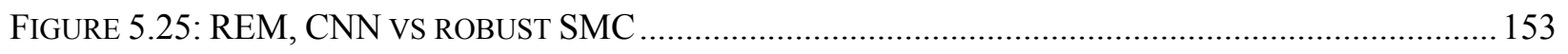

FIGURE 5.26: SYSTEM STATE SNAP SHOT AT DIFFERENT TIME, SPACECRAFT MASS $=50$ KG.................... 153

FIGURE 5.27 REFERENCE TRAJECTORY AND MINIMUM CONTROL INPUT REQUIRED ................................ 154

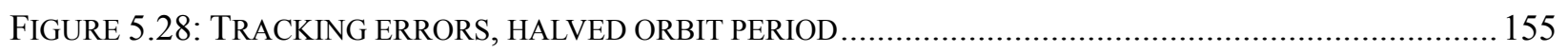

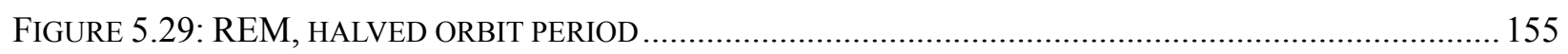

FIGURE 5.30 REFERENCE TRAJECTORY, ELLIPTICAL TO CIRCULAR ORBIT ................................................ 156

FIGURE 5.31 MINIMUM CONTROL INPUT REQUIRED, ELLIPTICAL TO CIRCULAR ORBIT............................. 156

FIGURE 5.32: SYSTEM STATE SNAP SHOT AT DIFFERENT TIME, ELLIPTICAL TO CIRCULAR ORBIT ............... 157

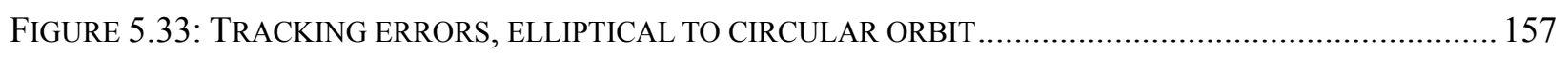

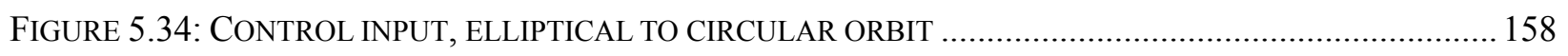

FIGURE 5.35: SYSTEM STATE SNAP SHOT AT DIFFERENT TIME, FORMATION RECONFIGURATION................ 159

FIGURE 5.36: STATE OF ALL AGENTS, FORMATION RECONFIGURATION ................................................... 159

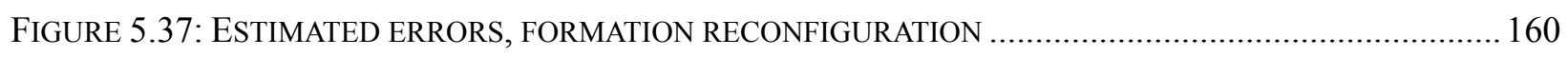

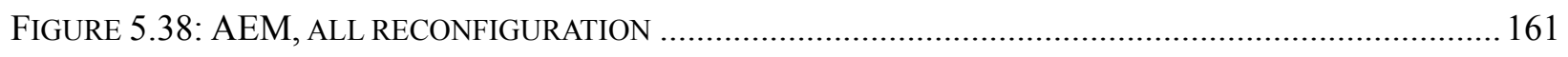

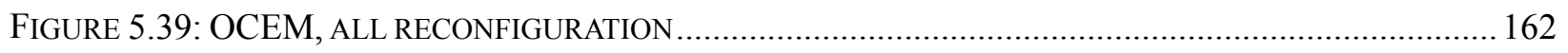




\section{List of Tables}

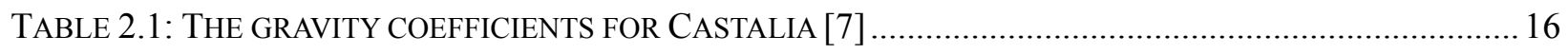

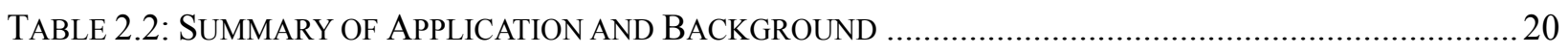

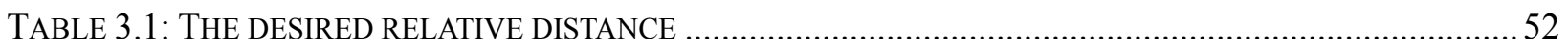

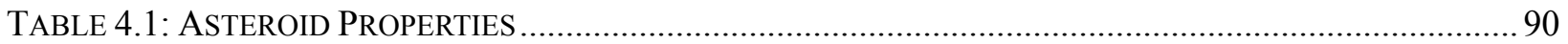



Space exploration has always been an interest of mankind. This thesis studies the application of exploring asteroids using multiple spacecraft by applying consensus formation flying technique. In decentralized formation flying, as opposed to centralized system, a more complicated communication network is required to model the system. The consensus algorithm is applied to achieve decentralized spacecraft formation flying. In addition, this study includes considerations about the effect of the body rotation, the $2^{\text {nd }}$ degree and $2^{\text {nd }}$ order gravity field, and the irregular shape of the astronomical body. The contribution of this thesis, is to apply the consensus algorithm with the use of nonlinear control, to facilitate multiple spacecraft orbiting around an asteroid. In this chapter, we support our research objective with a literature review; going over all aspects of relevant past and present publications and space missions. Problem statements and research objectives are presented to show the goal of this investigation. Lastly, we provide a thesis outline to make the thesis much easier to follow.

\subsection{RESEARCH Motivation}

"That's one small step for man; one giant leap for mankind." -Neil Alden Armstrong Mankind will never stop exploring the outreaches of the universe; we follow our ancestors' steps and go further out into space. There could be endless discussions about the reasons and merits of asteroid exploration, however, here are three fundamental reasons, which are also the motivation for the author's completion of this research: An eagerness to explore, wanting to understand the formation of the Solar System, and space mining.

This thesis studies the control of multiple spacecraft orbiting around an asteroid. The motion of spacecraft around an asteroid is different in character than that of the motion around the Earth, or other planetary bodies in the Solar system. There are hundreds and thousands of asteroids in space, and yet, there remains a lot for us to examine. In the literature, there exists no such studies regarding multi-agent consensus formation flying orbiting around an asteroid while using adaptive neural network control topology. In addition, NASA has recently proposed a novel ANTS mission 
that is sending thousands of spacecraft to the asteroid belt for investigation, which will be further explained in the literature review. These are the aspirations of the present thesis.

\subsection{LiTERATURE REVIEW}

An astronomical body, or celestial object is a naturally arising physical entity typically referring to a single, cohesive structure that is bound together by gravity. The planetary systems revolving around the Sun and building our Solar System are examples of celestial bodies. They can be described and characterized by their orbital mechanical properties and are categorized by the International Astronomical Union (IAU). Following the Committee on Small Body Nomenclature (CSBN), Minor Planets and Comets fall within the Small Body classification.

\subsubsection{Comets and Minor Planets}

A comet is a body composed of loose collections of rock, dust and ice particles, typically measuring a few kilometers in diameter, depending on its composition. Comets may pass by the Sun only once or go through the Solar System periodically, depending on their orbit and trajectory. A comet's tail is formed due to the effects of solar radiation and solar wind. This phenomenon can also be explained by the heat from the Sun warming the coma or nucleus of the comet, which then releases vapors into space.

Minor Planets are neither dominant planets nor comets, instead comprising of dwarf planets, asteroids, and other trans-Neptunian objects. Asteroids have no atmosphere and are made of rock and metal. On average, asteroids are closer than trans-Neptunian objects and have different orbital characteristics than dwarf planets.

Over 250,000 asteroids have been designated [1] and only around 100,000 have been observed. There are four major districts where asteroids are located, and they are defined by their distance from Earth. Near-Earth asteroids are around 1.0 AU from Earth, Main Belt asteroids are located between the orbits of Mars and Jupiter; Trojan asteroids are positioned at the Lagrangian points, which are 60 degrees ahead and behind Jupiter; and Centaurs asteroids are found in the outer solar system, orbiting around Mars and beyond Neptune [1]. The collaboration of multiple small space vehicles is proposed by many researchers in order to reduce cost and complexity of the system but maintain competence to satisfy modern space missions. 
Small solar system bodies have always been of great interest to space scientists, however, engineers encounter significant dynamical challenges when conducting space missions to explore them. Alternately, small near-Earth asteroids (NEAs) are the most accessible bodies in the solar system for low-cost space missions with regard to propellant. Therefore, asteroids are likely the next milestone in space exploration. The study of small bodies also provides advantages such as supplying valuable information about the formation of the Solar System and Earth, preparing for strikes on the planet by tracking their positions, and harvesting minerals from small astronomical bodies supported by the advanced technology.

\subsubsection{Past and current challenges}

As presented on the NASA Jet Propulsion Laboratory Solar System Dynamics website, the most updated counts of comets and asteroids as of May 24 $4^{\text {th }}, 2013$ are around 3,200 and 615,400 respectively [2]. Due to the vast amount of small bodies, the study of their behaviors needs to be filtered. For the purpose of this paper, from this point forth, an "asteroid" will be referred to as a "small body". These small celestial bodies are scattered through space, and are self-explained by their names; Near-Earth asteroids, Mars-Crossers asteroids, Asteroid-Belt asteroids, Trojan asteroids, Outer-Planet Crossers minor planet, and Centaurs. While there are a number of similar characteristics to consider when studying small astronomical bodies, the focus of this study is on their irregular shapes, rotation poles and mass distributions.

\subsubsection{Previous study about asteroid orbital dynamics}

Hudson and Ostro have stated the shape of Asteroid 4769 Castalia and the shape and nonprincipal axis spin state of Asteroid 4179 Toutatis in the mid 1990s [3, 4] from the observation of their radar images. Dynamic equations that describe the orbital motion close to asteroid 4769 Castalia are given in [5]. Farinella, Paolicchi and Zappala analyzed the spin rate distribution of asteroids and they found that the average spin rate increases with size, whereas different taxonomic types have distinct aspects for their distributions. These observations can be interpreted in terms of a complex and size-dependent collisional history of asteroids[6]. According to astronomers, the rotation and orbits of celestial objects are largely determined by the conservation of angular momentum. Star systems are always spinning as nebulas form, and momentum is lost when the nebula collapses on itself. The remaining momentum transfers into the new body form as a result 
of the rotations. The direction of the rotation also depends on the gravitational field acting on the object and other conditions as each astronomical body is formed and enters its orbit.

Asteroids and comets are located in different locations in our Solar System, and all celestial bodies are experiencing various forces. Several researchers have examined the problem of orbital dynamics around asteroids [7-9]. General formulation of the orbital dynamics around uniformly rotating asteroids is presented by Scheeres [9]. The spacecraft orbital control was not considered. For this study, small bodies with particular characteristics were chosen to be investigated; for instance those small rotating astronomical bodies which are also experiencing the combination of relevantly significant Solar Radiation Pressure (SRP) force, primary body mass distribution, and Solar Gravity force.

\subsubsection{Spacecraft Formation Flying Missions}

With extensive study on the orbital dynamics around small bodies, certain missions have been flown and planned. In a mission that began in 2001, NASA's sample return probe, Genesis, is required to return samples of solar wind. In 2003, Hayabusa from the Japan Aerospace Exploration Agency collected samples and brought them back to Earth in the mission to Asteroid 25143 Itokawa. The Rosetta - ESA Comet Mission flew by asteroids Steins and Lutetia in 2004. As a result of the very weak gravitational field of most target comets, hence small dimensions (about $10 \mathrm{~km}$ in diameter) and low density (about $1 \mathrm{~g} / \mathrm{cm}^{3}$ ), a means of anchoring the spacecraft has to be provided to counteract forces and torques generated during sampling [10]. Chen, Baoyin and Li recently proposed that the spacecraft will pass through the main asteroid belt between the orbits of Mars and Jupiter, and may encounter multiple asteroids [11].

\subsubsection{Centralized formation flying}

The investigation of controlling a monolithic spacecraft has passed its prime. From the mid to end of the twentieth century, studies were conducted to investigate how to distribute an array of small, low-cost , cooperative, and highly coordinated micro-spacecraft [12, 13], hence the use of centralized formation flying $[14,15]$. Several centralized formation flying space missions were pursued, and they can be classified into two common manners, trailing formations and cluster formations.

As defined by its name, trailing formations are multiple spacecraft shadowing one another by sustaining a specific interval orbiting on the same path. Remarkable pairs such as Landsat 7 
with Earth Observing-1 (EO-1) satellite enable scientists to study high-resolution images and climatic trends in the Earth's Environment [16]; the Calipso with CloudSat mission analyzed nearly the same volumes of the atmosphere within 10 - 15 seconds of each other by a tight orbital configuration $[17,18]$; and Terra with Aqua launched in, December $18^{\text {th }}, 1999$ and May $4^{\text {th }}, 2002$ respectively, carrying the major NASA Earth Observing System (EOS) instrument, the Moderate Resolution Imaging Spectroradiometer (MODIS). These twin satellites complement each other by providing a long term data set with the same geophysical parameters for the study of climate and global change studies with observations in the late morning and early afternoon [19-21].

On the other hand, cluster formations are constructed by spacecraft in a relatively tightly spaced array which require advanced science and technology in understanding the dynamics of spacecraft in close proximity to each other. Cluster II is one of the outstanding space missions using tetrahedron formations to explore the Sun-Earth connection, especially the interaction of the Sun and the Earth's magnetosphere. These [22-24] prove that the cluster formation can be intelligently simulated, controlled and applied.

\subsubsection{Decentralized formation flying}

In recent years, besides centralized formation flying, some attention has been steered toward formation flying using a decentralized network [25-32]. However, this innovative idea is not restricted to the space industry; it is also being used in wireless sensor/actuator networks, Cyber physical systems, telephone switchers, data categorizers, underwater swarm robots, and military defense taskforces [33-38]. This idea originates from natural science, through the observation of swarms of bees, flocks of birds, and schools of fish. When applied to space vehicle formation flying, a swarm can be defined as formation flying without a centralized scheme. Since there is no external force directing their behavior and no single agent has a global view of the intended macroscopic behavior, these types of swarms are totally self-organized and autonomous. Moreover, this complex collaborative behavior is formed by interactions among individuals that exhibit simple behaviors [39]. Curtis et al. from NASA Goddard Space Flight Center have proposed the use of swarm intelligence in spacecraft constellations for the resource exploration of the asteroid belt [40], the Autonomous Nano-Technology Swarm (ANTS). ANTS is targeting thousands of widely separated bodies in the Main Belt, which will require hovering in a highly irregular gravity field. 
This swarm concept has been brought to several missions and mission statements. The system is to conduct itself autonomously to complete the global objective, and each specialized agent is necessitated to work both individually and collectively to accomplish the local objective. The particularized agents in the ANTS mission can be heterogeneous or homogeneous. Since they are working in different environments they may learn different things, even if the squad starts out as homogeneous. Self-Configuring, Self-Optimizing, Self-Healing, and Self-Protecting are the four required autonomic properties for ANTS to approach total autonomy [41]. Artificial intelligence technologies such as genetic algorithms, neural networks, fuzzy logic and on-board planners are being applied to assist the mission in maintaining a high level of autonomy. To examine an asteroid in the belt, sub-swarms will form and act as teams that explore a particular asteroid. Teams will have to share resources due to the limited quantity of miniature instruments carried on-board in these pico-satellites [42]. There will be several types of spacecraft involved in the mission. As defined by Rouff, workers have specialized instruments on-board (e.g. a magnetometer, X-ray, gamma-ray, visible/IR, neutral mass spectrometer etc.) that perform telemetric operations; coordinators or rulers follow conditions to determine the types of asteroids the mission focuses on and allocate deliverables to the workers; and messengers coordinate communications between the workers, rulers, and ground stations on Earth.

Communication is the key to success in an autonomous swarm mission. Consensus control indicates that different agents make informed decisions toward the global objective by knowing what the other agents know [38]; each agent must have the same overall goal, know where the other agents are, and hence share its position and then decide on its own action. Four steps, proposed by Ren and Beard [43], which will assist in setting up the control law include: appointing a cooperation objective, selecting the required information, defining a centralized strategy, and building a consensus algorithm.

\subsubsection{Consensus Spacecraft Formation Control}

Throughout the literature $[25,26,28-37,44,45]$, it has been assumed that the agent dynamics on consensus control for multi-agent systems are known. A common method used to obtain unknown states of a system is an adaptive law. Two of the most investigated adaptive laws are neural networks and fuzzy logic systems [46-50]. They both have the ability to approximate any smooth functions with a high nonlinear regression, which makes them the best candidates to 
estimate the consensus control for multi-agent systems with uncertain nonlinear dynamics. In [51], a neural network adaptive control is used in a leader-follower control of second-order systems with uncertain dynamics and external disturbances. All states are assumed to be obtainable for all agents within the network. Furthermore, leaderless consensus control with neural network-based decentralized control topology is proposed in [52]. In [53], an adaptive neural controller for the consensus tracking of second-order multi-agent nonlinear systems in the presence of unknown disturbances has been investigated. A more contemporary study [54] has utilized a neural networkbased distributed tracking control scheme for unknown Lagrangian systems. Even though adaptive control is proposed in $[51,53,54]$, their study is focused only on a subset of group agents who has access to mutual desired trajectory, and the states are shared among each agent. The Chebyshev neural network is a functional link network whose input is generated by using a subset of Chebyshev polynomials, and it has been shown that CNN has powerful estimation proficiencies, see [55] and [56].

The establishments of this thesis includes the development of new control law to achieve stable orbital motion. Moreover, analysis of orbit stability for spacecraft orbiting rotating asteroids using numerical simulation is conducted and the result shows the natural response of the spacecraft orbit with the predefined reference trajectory. Furthermore, examination of a new orbital control law is conducted on a system model for asteroids using numerical simulation. Additionally, based on the algorithm states on [43], a consensus network for multiple space vehicles is developed to orbit around a slow rotating asteroid with the equation of motion established by $\mathrm{Hu}$ et al. [8]. We also investigate the effect on different formation configurations in an elliptical reference trajectory.

\subsection{PROBLEM STATEMENT}

Reviewing the literature presented in the previous section gives a clear picture of the developments that have occurred in the spacecraft asteroid orbiting and formation control discipline. More intriguing, however, is the lack of work primarily in the area studying the autonomous consensus algorithm for spacecraft formation flying and how it can be implemented during various phases of a mission.

An asteroid might have an irregular shape, at which point the moment of inertia and mass distribution need to be considered. The rotation rate of the asteroid is more influenced by its geometry. The gravitational field will vary within the tracking orbit. Besides the orbital dynamic, 
another major difference between orbiting a planet and orbiting an asteroid is the altitude of the orbiter in each situation. For example the low Earth orbit (LEO) is an orbit with an altitude of 160 $\mathrm{km}$ to 2,000 km above Earth, however, the most proposed altitude for orbiting an asteroid is just 10 to $20 \mathrm{~km}$.

In order to handle the highly complex and nonlinear dynamic system, the traditional linear controller is known not to be as efficient as the nonlinear controller. Moreover, from a space mission point of view, the system dynamics might not be known in advance or they might have more uncertainty than expected, especially when flying in the highly populated asteroid belt.

Asteroid observation with a monolithic spacecraft, which is required to carry all of the massive equipment for a complicated mission, will lose its agility to maneuver in the asteroid belt. In these cases, formation flying is proposed, the leader-follower formation might help to increase the flexibility of the system. Nonetheless, the success rate of the mission might not improve largely because the mission might not be able to continue if the leader is destroyed.

Many substantial trials must be overcome before an autonomous consensus control with the use of Terminal Sliding Mode Control (TSMC) for spacecraft can be turned into a practical mission. The problem statements for this thesis can be listed as:

[PROB1] Nonlinear spacecraft formation flying models. The fundamental nonlinearity in the orbital and formation dynamics of spacecraft is identified as one of the scientific problems to surmount for successfully developing multi-agent consensus control. The dynamic behavior of spacecraft for a broad range of operating states cannot be precisely defined by a linear mathematical model.

[PROB2] Model uncertainties and external disturbances. In any control applications, it is nearly impossible to define a perfect model to encounter all possible dynamic situations. The controllers designed based on nonlinear models can also be inaccurate due to unidentified assessments of some physical parameters, disturbance models, etc. The resulting mismatch between the model and the real system is referred to as model uncertainty. The problem of disturbance for the current application is particularly welldefined for situations where they operate in locations where their dynamics are affected by solar wind, solar radiation pressure and/or solar gravity. 
[PROB3] Control input saturation. There must be a limit on the amount of force the onboard actuator can provide. Particularly, modern satellites are more compact, and in return, the output force they can provide is relatively less than before. Saturation is unfavourable for space systems because the continuous presence of actuator saturation can cause extensive performance degradation lead to unstable system. For the occasion of spacecraft tracking, input saturation can be a major factor in tracking errors, along with parameter uncertainties and various sources of internal and external disturbances.

[PROB4] Multi-agents formation. The basic elements for formation flying technology can be classified as sensing, communication, and control. One of the biggest challenges between single spacecraft and multiple-agent formation flying is collision avoidance. In order to avoid collisions, the entire system has to maintain the right orbit and the right formation simultaneously, which relies on the control of the absolute and relative position.

It is understandable that the challenges illustrated above are correlated and the construction of a robust controller will be completed if these issues are taken care of explicitly. Since the mission to orbit around asteroids is still relatively new according to the knowledge of this research field, modeling uncertainty is required to increase the success rate for such delicate missions. If the integrity of the control law is not designed resiliently, the formation of the agents, the local task and/or the global mission will not be accomplished successfully.

\subsection{RESEARCH OBJECTIVES}

In response to the problem statement described above, this thesis focusses on the design of an adaptive control law, the end product of which is comprised of the use of consensus algorithm Chebyshev Neural Networks, robust control, and the terminal sliding mode control for spacecraft formation flying orbiting around a slowly rotating asteroid with irregular geometry. A consensus algorithm is integrated to improve the integrity of the formation flying between multiple agents. A control law is meticulously designed to suit the need for controlling both absolute and relative tracking errors. Neural networks are put into operation to contend with possible uncertainty, and the robust control law is used to make the controller more robust. 
From a practical viewpoint, our goal is to design a control system that can control multiple agents simultaneously in the presence of model uncertainties and large disturbances. The following objectives are identified:

[OBJ1] Decentralized formation flying. Leader and follower systems are generally, but not limited to, two spacecraft, they are limited to one task, on one mission, for one leaderfollower system. Centralized formation flying shares global team knowledge, and is a fully connected network. With consensus control, the resources are not restricted to a singular system; they involve neighbour to neighbour interaction. A system within a system can be setup for a more complex mission.

[OBJ2] Adaptive control for uncertain system model. Develop a reconfigurable control strategy based on adaptive control theory, in which the adaptive control structure implicitly reconfigures the control law using adaptive estimates of spacecraft dynamics during flight. With the use of the Chebyshev polynomial, this allows the controller to constantly update its parameters using an adaptation mechanism. The objective is to provide autonomous recovery using a reliable and effective control law that accounts for modeling uncertainties, external disturbances, and actuator failures simultaneously.

[OBJ3] Robust control for external disturbances. There are unavoidable disturbances, especially for the current proposed mission. Such as the environmental and non-environmental disturbances due to the location of the asteroid and the relative position of the Sun. Robust control can respond to bounded disturbances. In addition, from a numerical standpoint, it is known that Sliding Mode Control (SMC) with the use of the numerical sign function will impose a chattering effect, and the hyperbolic tangent function is used in order to reduce this effect.

\subsection{MAIN CONTRIBUTIONS}

The main contributions of this thesis are:

1. A novel terminal sliding manifold based on lumped state errors is proposed for the consensus tracking control of second-order multi-agent systems. Additionally, the proposed controller is non-singular because no negative fractional powers exist in the proposed controller. 
2. As compared with the existing approaches $[54,57,58]$ on consensus tracking control of second-order multi-agent systems in case only a subset of group members has access to the leader agent, the system states information of neighbour agents is not required in the proposed control law, which can reduce both the cost related to sensors and information flow between neighbour agents. As compared with the terminal sliding mode-based consensus tracking controller proposed in [45], control signals are not necessary for exchange between neighbour agents, which not only reduces the information flow between neighbour agents but also avoids the algebraic loop problem existing in the controller proposed in [45].

3. In contrast to the existing approaches on the consensus for networks of non-identical agents [59-61], the leaderless consensus problem was studied in [59,60] while the leader-follower consensus problem is addressed in [55]. Although the leader-follower consensus problem was studied in [61] for affine multi-agent systems, the time-varying synchronized trajectory needs to be the output of a known linear system, and the knowledge of the synchronized signal is assumed to be available to each agent in the group. Furthermore, both system uncertainties and external disturbances are not considered in [59,60], and only parametric uncertainties are taken into account in [61]. However, both system uncertainties and external disturbances are explicitly considered in designing the distributed controller in the present dissertation.

4. The robust controller using the hyperbolic tangent function is used to counteract CNN approximation errors and bounded external disturbances, and hence, the chattering caused by the use of numerical sign function in the existing TSMC approaches $[45,55]$ can be avoided.

5. Finite-time stability in both the reaching phase and the sliding phase is proven by the Lyapunov approach.

\subsection{Thesis OUtLINE}

This thesis is comprised of four main sections, followed by a conclusion and is mapped out systematically. In Chapter 2, the asteroid orbital dynamic system is discussed. It presents a formulation of the spacecraft moving in an elliptical orbit around a small body and the Lagrangian method is used to obtain the governing ordinary differential equations of motion for the system. This is followed by Chapter 3, where the nonlinear SMC is applied to the closed-loop system and examined with the Lyapunov stability analysis. Chapter 4 contains a robust control law designed to counter a bounded disturbance. In Chapter 5 the dynamic system of the plant is not provided to 
the control law, and TSMC and CNN are applied and examined with the new control law to adapt to the new challenge. The governing equations of motion of the asteroid system are numerically integrated for each chapter. A detailed assessment of the system performance under the proposed control strategies, and the results of computer simulations incorporating different formation scenarios are also presented in each chapter. Finally, we conclude this thesis with a review of the motivation, objective, and results from each numerical analysis, and the contribution for the entire study. A discussion on future work is also included. 


\section{SYSTEM MODEL AND MULTI-AGENT SYSTEM}

The multi-agent system is one of the key technologies of current and future space missions. The main challenge is to control the relative positions of spacecraft in formation when the external disturbances cause drifts of both the relative positions of the spacecraft and the formation center. In this chapter, the mathematical model of the spacecraft formation orbiting around an asteroid is introduced. Furthermore, the coordinate frames defining the dynamics of the spacecraft orbital motion, and the time-varying equations describing the elliptical reference trajectory are shown. In addition, external disturbance models and minimum control input required for tracking the proposed trajectory are presented. Moreover, some basic knowledge of graph theory and the properties of the matrix that will be used are provided. Lastly, the system error model that applies the consensus algorithm to decentralized formation flying is presented.

\subsection{SYSTEM MODEL}

Spacecraft orbiting around an asteroid is different from the Keplerian orbit in the two-body problem. This occurs for two reasons, one being the irregular shape of the body, and the second the rotation of the asteroid. These coupling effects can lead to large energy and angular momentum changes within short time periods. Alternatively, these effects are not dominant in case of spacecraft motion around planetary bodies.

In a general two-body system, when the spacecraft is assumed to be a point mass, its position vector in inertial coordinates satisfies the equation of motion,

$$
\ddot{\boldsymbol{r}}=-\frac{\mu}{r^{3}} \boldsymbol{r}
$$

where $\boldsymbol{r}$ is the relative position vector between two masses, and $r=\|\boldsymbol{r}\|$, the magnitude of $\boldsymbol{r}$. The gravitational parameter, $\mu$, is defined as

$$
\mu=\mathrm{G}\left(m_{1}+m_{2}\right)
$$


For the above equation, $m_{1}$ and $m_{2}$ are masses of the two bodies in the system, and $G$ is the universal gravitational constant, where $G=6.67384 \times 10^{-11} \mathrm{~m}^{3} \mathrm{~kg}^{-1} \mathrm{~s}^{-2}$.

\subsubsection{Spacecraft Orbital Dynamics Around an Asteroid}

The orbital motion is stable around a symmetrical celestial body when it remains near to its unperturbed orbit, conventionally. A more specific type of orbital stability is defined in [7] for asteroid stability analysis. When orbiting near the surface of an asteroid that is rotating slowly, these orbits are usually in a size-shape stable region. When orbits are far away and the asteroid rotates rapidly, then these orbits are also usually in the stable region. However, in the resonance region, the orbits are usually unstable and these orbits are said to be size-shape unstable.

A two-body problem, or an unperturbed Keplerian orbit, is the hypothetical motion that the body follows under the gravitational effect of only one other body, which usually can be described with the method of geometry. We commonly call this the orbital elements. In an unperturbed Keplerian orbit, the mean anomaly, the argument of perigee, $\omega$; the longitude of ascending node, $\Omega$; the inclination, $i$; the eccentricity, $e$; and the semi-major axis, $a$, will remain unchanged, where the first three orbital elements belong to the orientation group and the last two elements are classified as the size-shape group. For example, when orbiting around an oblate planet; i.e. Earth with J2 effect, the average orbit's size and shape are constant, however, the orientation elements change. In contrast, when orbiting around small bodies, all elements change, and that increases the complexity when studying the orbital dynamics around an asteroid. With the above definition, it is clear that orbit is size-shape stable when semi-major axis and eccentricity are constant, on average, and we call it the nominal orbit. On the other hand, when these two orbital elements are not constant, on average, we call it size-shape unstable. Frequently, we describe this motion as chaotic. As studied in [7], when the orbit motion is chaotic, the semi-major axis and eccentricity rapidly depart from their initial specification and oscillate about their nominal values.

The study in [7] has also found that the stability of an orbital motion will be more sizeshape stable when the orbiting body is far from the asteroid due to a weaker perturbation force applied from the small body to the orbiting body. Radius is a factor of the strength of the perturbation, however, the rotation rate, $\omega_{T}$, and the gravity coefficient, $C_{22}$, are important, too.

Resonance always leads to chaotic orbit, and it occurs when the ratio of the asteroid rotation rate, $\omega_{T}$, and the nominal mean motion, $n_{0}$, of an orbiting body are near a rational number. There are 
two cases in which the orbits are size-shape stable. The first occurs when the orbits are near the asteroid and the asteroid rotates slowly where $\left|\left(n_{0}-\omega_{T}\right) / \omega_{T}\right| \gg 1$. The second occurs when the orbits are far from the asteroid and the asteroid rotates rapidly, and $\left|\left(\omega_{T}-n_{0}\right) / n_{0}\right| \gg 1$.

\subsubsection{System Dynamics Model}

The spherical harmonic expansion of a gravitational force potential [7] is defined in Equation (2.3)

$$
U_{g}=\sum_{l=0}^{\infty} \sum_{m=0}^{l} \frac{\mu}{r^{l+1}} P_{l}^{m}(\sin \delta)\left[C_{l m} \cos (m \lambda)+S_{l m} \sin (m \lambda)\right]
$$

where $P_{l}^{m}(\sin \delta)$ is the Associated Legendre Functions of order $l$ and degree $m, \mu$ is gravitational parameter, $r$ is the radius measured from the center of mass of the body, $\delta$ is the particle declination measured from the $\mathrm{x}-\mathrm{y}$ plane, which is defined in Figure 2.1, and $\lambda$ is the particle longitude in the body-fixed frame.

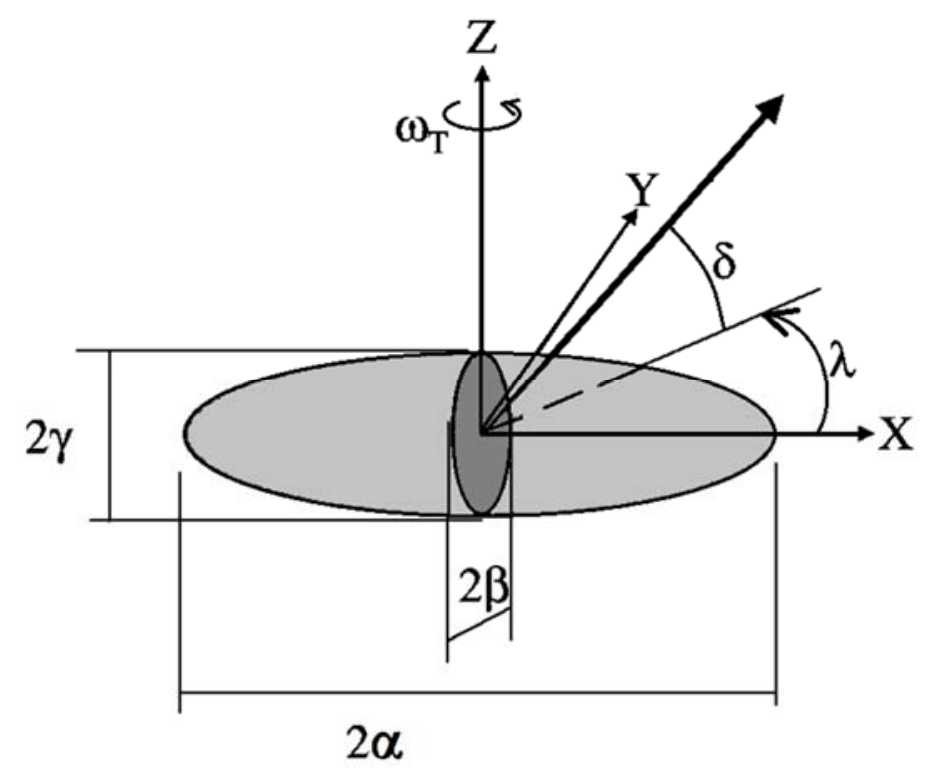

Figure 2.1 Position geometry of a point mass of an asteroid in the body fixed space [8] 
The Legendre ordinary differential equation occurs when solving Laplace's equation in spherical coordinates. The general form of Associated Legendre Functions, with the application of Rodrigues' formula, yield

$$
P_{l}^{m}(x)=\frac{\left(1-x^{2}\right)^{m / 2}}{2^{l} l !} \frac{d^{l+m}}{d x^{l+m}}\left(x^{2}-1\right)^{l}
$$

Substitute $\sin \delta$ into $x$, and simplified

$$
\begin{aligned}
P_{l}^{m}(\sin \delta) & =\frac{\left(1-(\sin \delta)^{2}\right)^{m / 2}}{2^{l} l !} \frac{d^{l+m}}{d(\sin \delta)^{l+m}}\left((\sin \delta)^{2}-1\right)^{l} \\
& =\cos ^{m} \delta \sum_{t=0}^{k} T_{l m t} \sin ^{l-m-2 t} \delta
\end{aligned}
$$

where $k$ is the integer part of $(l-m) / 2$, and $m \leq l . T_{l m t}$ is an integer which is given by

$$
T_{l m t}=\frac{(-1)^{t}(2 l-2 t) !}{2^{l} t !(l-t) !(l-m-2 t) !}
$$

Continuing with Equation (2.3), $C_{l m}$, and $S_{l m}$ are the gravity coefficients. To be more specific, $C_{l 0}$ are called zonal harmonic coefficients, and the gravity coefficients are called sectorial harmonic coefficients when $l=m$, and tesseral harmonic coefficients when $l \neq m$. The gravity coefficients for Castalia through degree 4 are shown in Table 2.1. Notice that the magnitudes of $C_{20}$ and $C_{22}$ are much larger than the other coefficients.

Table 2.1: The gravity coefficients for Castalia [7]

\begin{tabular}{|c|c|c|c|}
\hline Degree $(l)$ & Order $(m)$ & $C_{l m}$ coefficient & $S_{l m}$ coefficient \\
\hline 0 & 0 & 1.0 & - \\
\hline \multirow{3}{*}{1} & 0 & 0.0 & - \\
\cline { 2 - 4 } & 1 & 0.0 & 0.0 \\
\hline \multirow{3}{*}{2} & 0 & -0.110298 & - \\
\cline { 2 - 4 } & 1 & 0.0 & 0.0 \\
\cline { 2 - 4 } & 2 & 0.156733 & 0.0 \\
\hline
\end{tabular}




\begin{tabular}{|c|c|c|c|}
\hline \multirow{3}{*}{3} & 0 & -0.015112 & - \\
\cline { 2 - 4 } & 1 & -0.037935 & 0.001211 \\
\cline { 2 - 4 } & 2 & 0.006325 & 0.000616 \\
\cline { 2 - 4 } & 3 & 0.020568 & -0.013715 \\
\hline \multirow{4}{*}{4} & 0 & 0.036630 & - \\
\cline { 2 - 4 } & 1 & 0.002706 & 0.000407 \\
\cline { 2 - 4 } & 2 & -0.051363 & 0.003949 \\
\cline { 2 - 4 } & 3 & 0.006140 & -0.001747 \\
\cline { 2 - 4 } & 4 & 0.050334 & -0.006839 \\
\hline
\end{tabular}

The perturbation terms from the asteroid gravity field is an important factor when studying spacecraft dynamics orbiting around asteroid. Even though only the $2^{\text {nd }}$ degree and $2^{\text {nd }}$ order gravity coefficients, $C_{20}$ and $C_{22}$, respectively, will be used in our investigation, we provide a few remarks about the higher order gravity potential expansions.

Using Equation (2.5) and (2.6), we calculate the spherical harmonic expansion of a gravitational force potential up to the 4th-order.

$$
\begin{aligned}
& U_{g}=U_{g 0}+U_{g 1}+U_{g 2}+U_{g 3}+\cdots U_{g n} \\
& U_{g 0}=\frac{\mu}{r} \\
& U_{g 1}=\frac{\mu}{r^{2}}\left[C_{10} \sin \delta+\cos \delta\left(C_{11} \cos \lambda+S_{11} \sin \lambda\right)\right] \\
& U_{g 2}=\frac{\mu}{r^{3}}\left[C_{20}\left(1-\frac{3}{2} \cos ^{2} \delta\right)+\frac{3}{2} \sin (2 \delta)\left(C_{21} \cos \lambda+S_{21} \sin \lambda\right)\right. \\
& \left.\quad+3 \cos ^{2} \delta\left(C_{22} \cos 2 \lambda+S_{22} \sin 2 \lambda\right)\right]
\end{aligned}
$$




$$
\begin{aligned}
U_{g 3}=\frac{\mu}{r^{4}}\left[C_{30}\right. & \sin \delta\left(1-\frac{5}{2} \cos ^{2} \delta\right) \\
& -\frac{3}{2}\left(1-5 \sin ^{2} \delta\right) \cos \delta\left(C_{31} \cos \lambda+S_{31} \sin \lambda\right) \\
& +15 \sin \delta \cos ^{2} \delta\left(C_{32} \cos 2 \lambda+S_{32} \sin 2 \lambda\right) \\
& \left.+15 \cos ^{3} \delta\left(C_{33} \cos 3 \lambda+S_{33} \sin 3 \lambda\right)\right] \\
U_{g 4}=\frac{\mu}{r^{5}}\left[C_{40}\right. & \left(\frac{35}{8} \sin ^{4} \delta-\frac{15}{4} \sin ^{2} \delta+\frac{3}{8}\right) \\
+ & \left(\frac{35}{2} \sin ^{3} \delta-\frac{15}{2} \sin \delta\right) \cos \delta\left(C_{41} \cos \lambda+S_{41} \sin \lambda\right) \\
+ & \left(\frac{105}{2} \sin ^{2} \delta-\frac{15}{2}\right) \cos { }^{2} \delta\left(C_{42} \cos 2 \lambda+S_{42} \sin 2 \lambda\right) \\
+ & 105 \sin ^{2} \cos ^{3} \delta\left(C_{43} \cos 3 \lambda+S_{43} \sin 3 \lambda\right) \\
+ & \left.105 \cos ^{4} \delta\left(C_{44} \cos 4 \lambda+S_{44} \sin 4 \lambda\right)\right]
\end{aligned}
$$

The gravitational potential can be simplified by the following assumptions. These simplifications are practical and commonly used [7, 8, 62].

Assumption 1: In the gravitational potential, the magnitude for the second order, second degree terms are more significant than the higher order, higher degree terms; therefore, the higher degree, higher order terms are neglected.

Assumption 2: The coordinate frame is at the center of mass of the asteroid; that implies

$$
C_{10}=C_{11}=S_{11}=0
$$

Assumption 3: The body fixed coordinate axes are assumed to be aligned along the body's principal moments of inertia, hence

$$
C_{21}=S_{21}=S_{22}=0
$$


The gravity coefficient can be expressed with the principal moments of inertia of the body normalized by the body mass. In addition, the principal moments of inertia for an asteroid, or the more general term irregular shaped celestial body, are ordered as , $I_{x x}<I_{y y}<I_{z z}$, so that $C_{20}<$ 0 , and $C_{22}>0$.

$$
\begin{gathered}
C_{20}=-\frac{1}{2}\left(2 I_{z z}-I_{x x}-I_{y y}\right) \\
C_{22}=\frac{1}{4}\left(I_{y y}-I_{x x}\right)
\end{gathered}
$$

The equation of motion defined in Equation (2.1) is the ideal case where there are no external forces, disturbances, or perturbations. In a general case, this implies that the attracting body is a point mass; in a specific case, it can also be interpreted as a sphere with uniform mass distribution, with its principle moments of inertia, $I$, equaling, $I_{x x}=I_{y y}=I_{z z}$. The spacecraft

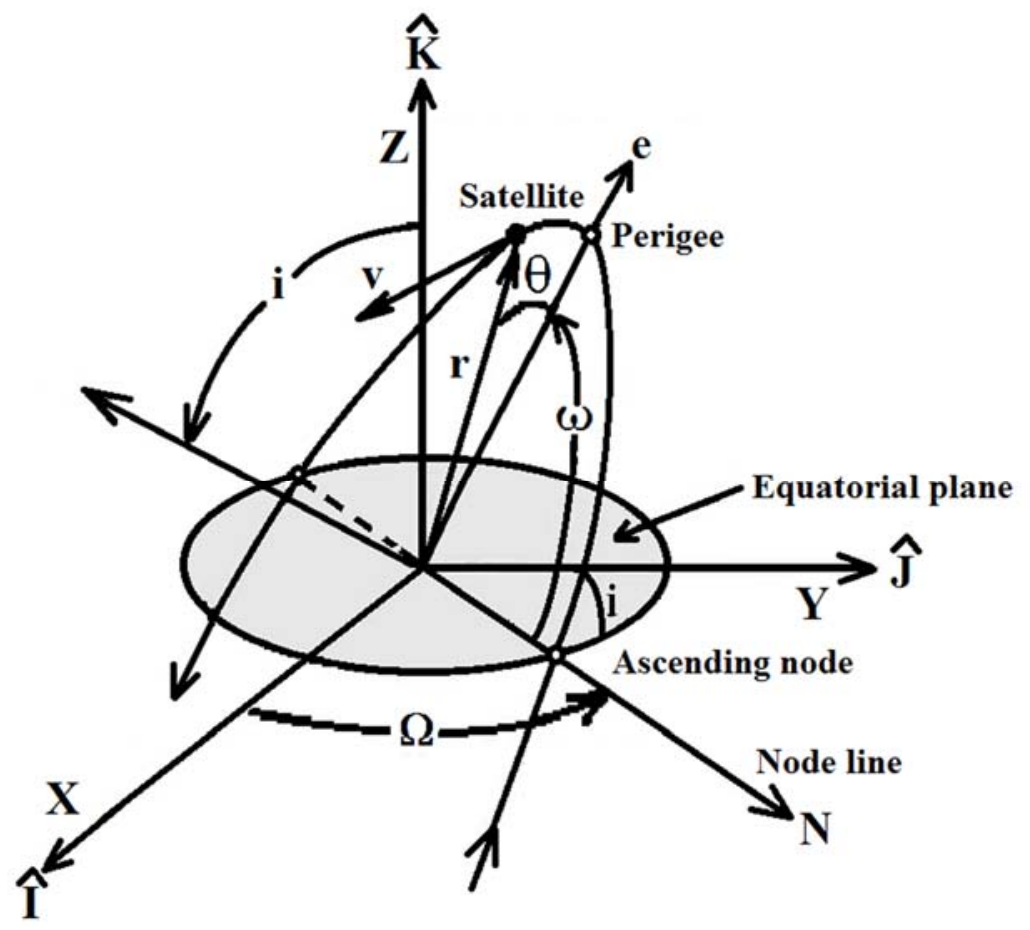

Figure 2.2 Geocentric equatorial frame and the orbital elements [63] 
orbit is said to be a classical Keplerian orbit where its inclination, $i$; eccentricity, $e$; longitude of ascending node, $\Omega$; semi-major axis, $a$; and argument of perigee, $\omega$, are all constant. These orbital elements are well defined in the Figure 2.2.

The moments of inertia for spacecraft motion around an asteroid are different from each other, $I_{x x}<I_{y y}<I_{z z}$, and the acceleration due to gravity between two masses is highly correlated with the asteroid's rotation. These characteristics make the study of motion around an asteroid completely different from the study of motion around an oblate body. The orbital elements will not be time invariant, the argument of perigee, $\omega$, and longitude of ascending node, $\Omega$, will change, and the inclination, $i$, eccentricity, $e$, and semi-major axis, $a$, will change even more significantly over time. The spacecraft motion will become unpredictable to some degree due to the rapid changes. This unpredictability is an instance of chaotic motion, which exists in many dynamic systems [7]. A summary of the previous discussion can be found in Table 2.2.

Table 2.2: Summary of Application and Background

\begin{tabular}{|l|c|c|c|}
\hline Central body shape & Sphere & Oblate & Irregular \\
\hline Moments of inertia & $I_{x x}=I_{y y}=I_{z z}$ & $I_{x x}=I_{y y}<I_{z z}$ & $I_{x x}<I_{y y}<I_{z z}$ \\
\hline Gravity coefficients & $C_{20}=C_{22}=0$ & $C_{20}<0, C_{22}=0$ & $C_{20}<0, C_{22}>0$ \\
\hline Relation to body rotation & No & No & Yes \\
\hline Motion constants & $\omega, \Omega, i, e, a$ & $i, e, a$ & None \\
\hline
\end{tabular}

In this dissertation, the asteroid we investigate are slowly rotating asteroids. The spacecraft motion is affected because of the gravity field is rotating with the asteroid. This brings some similarities to the three body problem, where the third body frame of reference is set as the inertial frame with a possible rotation in its axis [62].

\subsubsection{Equations of Motion in Body Fixed Coordinates}

In this section, we review some basic notations and equations that is used in the following chapters. The two-body system orbital equation of motion in an inertial coordinate is expressed in potential energy, $U_{g}$, with the position vector, $\boldsymbol{r}$, of the orbiting body

$$
\ddot{\boldsymbol{r}}=\frac{\partial U_{g}}{\partial \boldsymbol{r}}
$$


where gravity potential $U_{g}=\sum U_{g i}, i=0,1,2, \cdots n$. The equations are time varying if we include one of the important parameters of asteroid orbital dynamic, the angular velocity of the rotating asteroid. To reduce the complexity of the system, we transform Equation (2.17) into a rotating coordinate frame that is fixed to the small rotating celestial body. Let us first define, $\boldsymbol{r}=$ $\left[\begin{array}{lll}x & y & z\end{array}\right]^{T}, \boldsymbol{\omega}=\left[\begin{array}{lll}0 & 0 & \omega_{T}\end{array}\right]^{T}$, then

$$
\ddot{\boldsymbol{r}}+2 \boldsymbol{\omega} \times \dot{\mathbf{r}}+\boldsymbol{\omega} \times(\boldsymbol{\omega} \times \boldsymbol{r})=\frac{\partial U_{g}}{\partial \boldsymbol{r}}
$$

where $\omega_{T}$ is constant and $\dot{\boldsymbol{\omega}}=0$. The relation between the inertial coordinates and the body-fixed coordinates with rotation rate $\omega_{T}$ can be found in Figure 2.3. Assumptions are made following [8, 9], such that the asteroid is in uniform rotation about its maximum moment of inertia (the z-axis) with a rotation rate $\omega_{T}$ and a corresponding rotation period $T=2 \pi / \omega_{T}$.

The scalar form of Equation (2.18) can be found in [7], and are shown as follow

$$
\begin{gathered}
\ddot{x}-2 \omega_{T} \dot{y}=\omega_{T}^{2} x-\frac{\mu x}{r^{3}}+\frac{\partial U_{g 2}}{\partial x} \\
\ddot{y}+2 \omega_{T} \dot{x}=\omega_{T}^{2} y-\frac{\mu y}{r^{3}}+\frac{\partial U_{g 2}}{\partial y} \\
\ddot{z}=-\frac{\mu z}{r^{3}}+\frac{\partial U_{g 2}}{\partial z}
\end{gathered}
$$

Equations (2.19) to (2.21) contain the effect of coriolis and centripetal accelerations and the gravity potential, $U_{g 2}$, is defined in Equation (2.10). A more complete discussion of these equations as applied to motions about an asteroid appears in [9]. 


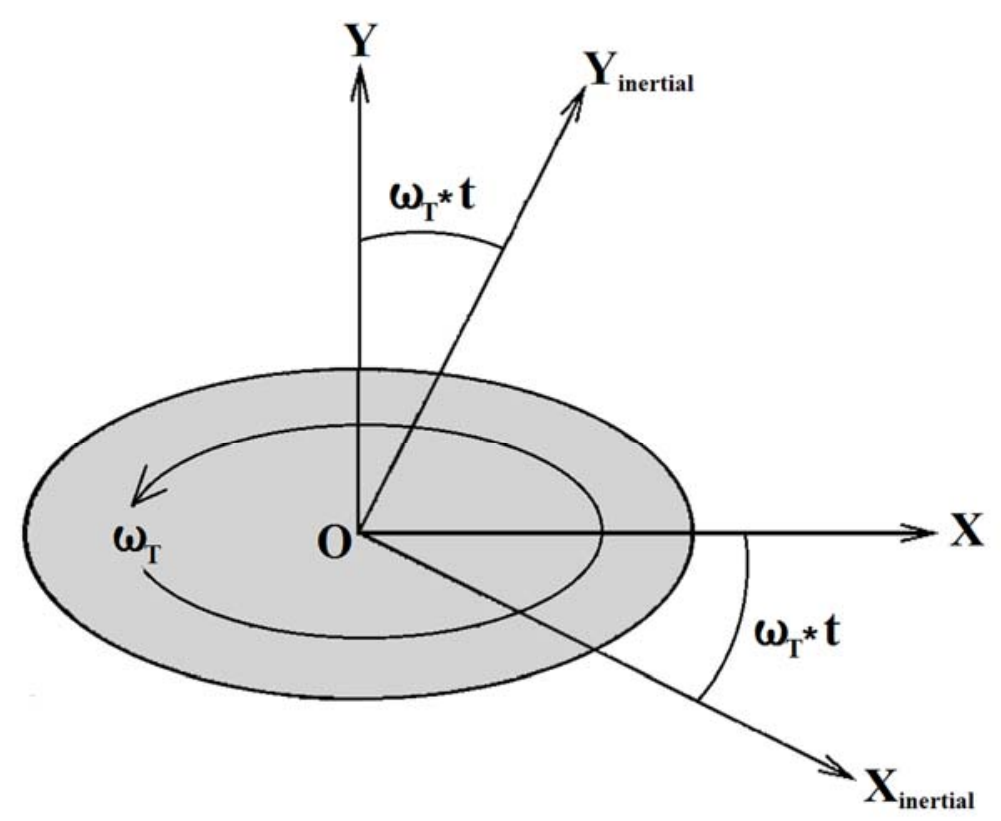

Figure 2.3 Geometry of the inertial and body-fixed coordinates

The $2^{\text {nd }}$ degree and $2^{\text {nd }}$ order gravitational perturbation potential in the body-fixed frame is expressed in the Cartesian coordinate as follows

$$
U_{g 2}=-\frac{\mu C_{20}\left(x^{2}+y^{2}-2 z^{2}\right)}{2 r^{5}}+\frac{3 \mu C_{22}\left(x^{2}-y^{2}\right)}{r^{5}}
$$

where $r=\sqrt{x^{2}+y^{2}+z^{2}}$ and the first order derivatives of the gravitational perturbation are

$$
\begin{gathered}
\frac{\partial U_{g 2}}{\partial x}=-\frac{\mu C_{20} x}{r^{5}}+\frac{5 \mu C_{20} x\left(x^{2}+y^{2}-2 z^{2}\right)}{2 r^{7}}+\frac{6 \mu C_{22} x}{r^{5}} \\
-\frac{15 \mu C_{22} x\left(x^{2}-y^{2}\right)}{r^{7}} \\
\frac{\partial U_{g 2}}{\partial y}=-\frac{\mu C_{20} y}{r^{5}}+\frac{5 \mu C_{20} y\left(x^{2}+y^{2}-2 z^{2}\right)}{2 r^{7}}-\frac{6 \mu C_{22} y}{r^{5}} \\
-\frac{15 \mu C_{22} y\left(x^{2}-y^{2}\right)}{r^{7}}
\end{gathered}
$$




$$
\frac{\partial U_{g 2}}{\partial z}=\frac{2 \mu C_{20} z}{r^{5}}+\frac{5 \mu C_{20} z\left(x^{2}+y^{2}-2 z^{2}\right)}{2 r^{7}}-\frac{15 \mu C_{22} z\left(x^{2}-y^{2}\right)}{r^{7}}
$$

These are the basic equations that describe the orbital dynamic around an asteroid. In our research, we start with this basic system for our first consensus control problem. Afterward, disturbance is introduced to the system to examine the capability of the controller.

\subsubsection{Reference Trajectory}

A reference trajectory demonstrates a suggested path by which the controlled variable should converge on the set-point in a particular manner. We can effectively define the closed-loop time response of the system by choosing the rate of convergence as a controller tuning parameter. The manner by which the final value is reached must also be taken into account. For example, the dynamic behaviour of the process that connects the initial to the final steady state value must be considered. It is possible to take many routes from point 1 to point 2, by geographical analogy, but some routes are more efficient and will be better than others. In the present investigation, an elliptical reference trajectory is considered. The trajectory uses in this thesis is obtained from Hill's equations [64].

In this formation, the orbital radius is defined as $x_{c}{ }^{2}+y_{c}{ }^{2}+z_{c}{ }^{2}=r_{d}{ }^{2}$, where $\left(x_{c}, y_{c}, z_{c}\right)$ are the desired or reference states. The relative motion in the radial/along-track (x/y) plane is fixed in eccentricity [64]. The equations of desired elliptical trajectory are given as follows,

$$
\boldsymbol{x}_{c}=\left\{\begin{array}{l}
x_{c} \\
y_{c} \\
z_{c}
\end{array}\right\}=r_{d}\left[\begin{array}{c}
\sin (n t) \\
2 \cos (n t) \\
\sqrt{3} \sin (n t)
\end{array}\right]
$$

where $r_{d}$ is the spacecraft altitude, and $n$ is the mean angular velocity that equals to $\sqrt{\mu / a_{c}^{3}} \cdot \mu$ is the gravitational parameter of the asteroid, and $a_{c}$ is the semi-major axis of the centre of the formation of the squad of spacecraft. Both, in reality or in numerical simulation, it is normal to choose a specific orbit period if the equilibrium orbit does not match the mission requirement. Throughout this thesis, various asteroids are investigated, however, the major focus of the investigation is about asteroid Castalia. In [65], Scheeres proposed having a reference orbit at a period of 8 times faster than the regular orbit for asteroid Castalia. The angular velocity, $\omega$, of this 
asteroid is $4.2883 \times 10^{-4} \mathrm{rad} / \mathrm{s}$, and the orbital period of the spacecraft orbit around an asteroid can be calculated using $2 * \pi / \omega$, which is approximately 4.07 hours. Following Scheeres's suggestion, the orbit period for the reference trajectory will be 0.5087 of an hour, approximately 30 minutes. Therefore, $n=2 * \pi / T_{p}$, where $T_{p}$ is one eighth of the orbital time period of the asteroid. This reference trajectory, shown in Figure 2.4, is used throughout this thesis unless stated otherwise. However, the control law developed are applicable to any formation flying and various spacecraft configurations.
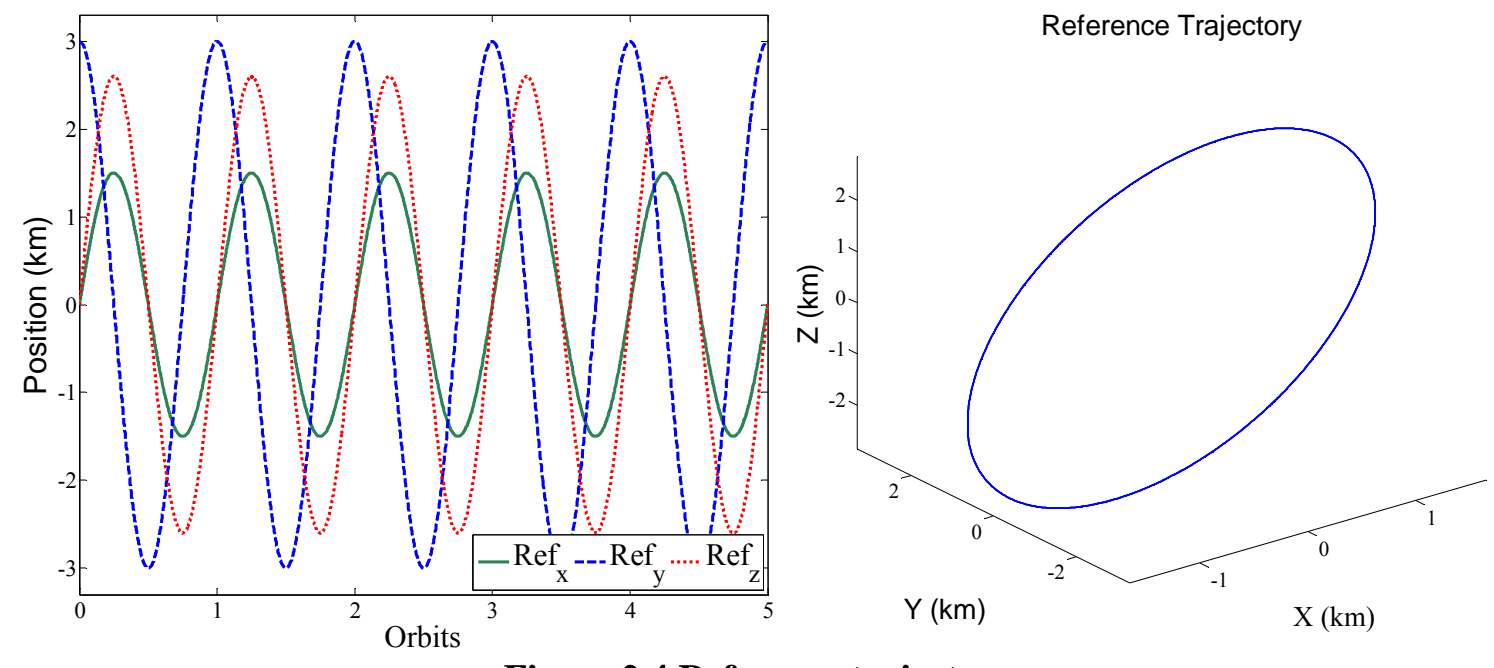

Figure 2.4 Reference trajectory

\subsubsection{External disturbances}

In this section, we summarize the suggested external force models that act on the spacecraft when orbiting around an asteroid. These include solar radiation pressure, and solar gravitational effects. In order to define these disturbances, we begin with a crisp review of the small body environment.

\subsubsection{Small body model}

Simple Keplerian dynamics are applied to model the astronomic small body orbital motion around the Sun. With the Keplerian model, the occurrence of a close passage by a planet is a scenario that rarely happens and will not be part of our analysis. More attention is brought to the varying position vector between the asteroid and the Sun, $\boldsymbol{d}[66]$, 


$$
\boldsymbol{d}=d \widehat{\boldsymbol{d}}=\frac{P}{1+e \cos v}(\cos v \hat{x}+\sin v \hat{y})
$$

where $P$ is the orbit parameter and $e$ is the eccentricity defined in the previous section. $v$ is the asteroid true anomaly. Both $\hat{x}$ and $\hat{y}$ are unit vectors, in which $\hat{x}$ is pointing to the orbit perihelion and $\hat{y}$ is in the heliocentric plane of motion and normal to $\hat{x}$.

\subsubsection{Solar radiation pressure}

Solar radiation pressure model is generalized based on the assumption that the principal motion for the spacecraft dynamics under the solar radiation force will not be changed significantly. For the ease of modeling, the spacecraft is modeled as a flat plate oriented to face the Sun and the absorbed solar radiation is radiated away uniformly. The force potential $S R P$ is formulated as [66]

$$
U_{S R P}=g \widehat{\boldsymbol{d}} \cdot \boldsymbol{r}
$$

where $g$ is the acceleration, $\widehat{\boldsymbol{d}}$ is the direction of the pressure acting away from the Sun, and $\boldsymbol{r}$ is the position of the spacecraft relative to the asteroid's center of mass. The disturbance force from the SRP can be computed by

$$
\boldsymbol{F}_{d(S R P)}=\frac{\partial\left(U_{S R P}\right)}{\partial r}=g \widehat{\boldsymbol{d}}
$$

in which the magnitude of the acceleration $g$ is formulated as

$$
g=\frac{(1+\rho) G_{1}}{B d^{2}}
$$

and $G_{1} \sim 1 \times 10^{8} \mathrm{~kg} \cdot \mathrm{km}^{3} /\left(\mathrm{s}^{2}-\mathrm{m}^{2}\right), B$ is the spacecraft mass to area ratio in $\mathrm{kg} / \mathrm{m}^{2}, \rho$ is the reflectance of the SRP on the spacecraft material, and $d$ is the distance between the Sun and the small body in $\mathrm{km}$. The magnitude of the SRP acceleration depends on the distance between the 
asteroid and the Sun, hence within the elliptical orbit, the SRP force changes between the minimum at aphelion and maximum at perihelion.

\subsubsection{Solar gravitation}

The solar gravitation is modeled as a third-body perturbation to the two-body system by taking the body-fixed coordinate on the asteroid as the center of our dynamical system. An appropriate expansion can be performed to simplify the functional form of such a disturbance. The perturbation potential from the Sun is expressed as follows [67]

$$
U_{S U N}=\mu_{S}\left[\frac{1}{|\boldsymbol{r}+\boldsymbol{d}|}+\frac{\boldsymbol{d} \cdot \boldsymbol{r}}{|\boldsymbol{d}|^{3}}\right]
$$

where $\mu_{s}$ is the gravitational parameter of the Sun, $\boldsymbol{r}$ is the position vector of the spacecraft relative to the asteroid, and $\boldsymbol{d}$ is the position vector of the asteroid relative to the Sun.

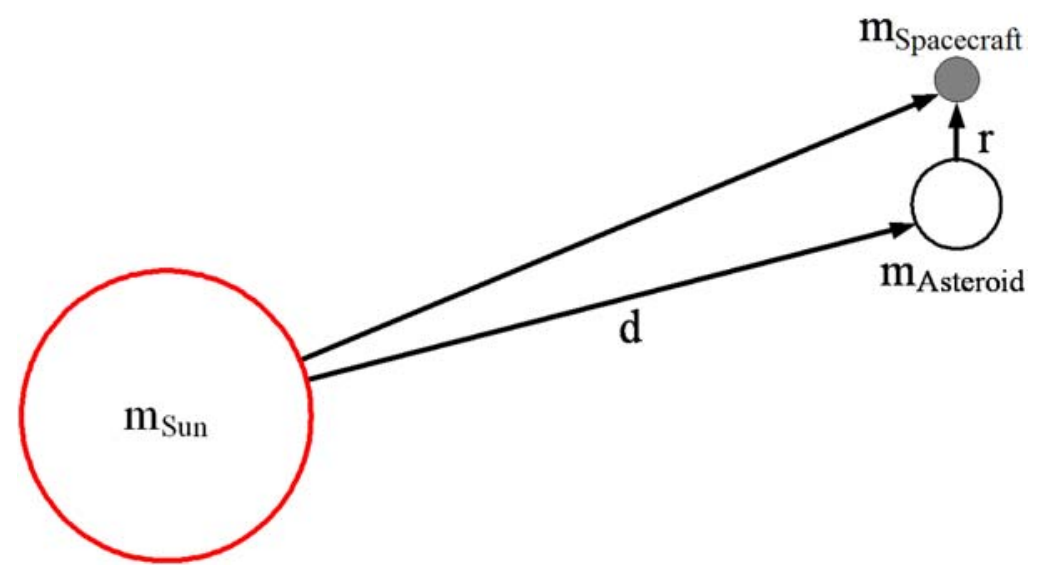

Figure 2.5 Relative position vectors in the Sun-Asteroid-Spacecraft system

The derivative of Equation (2.31) is taken with respect to $\boldsymbol{r}$ to find the disturbing gravitational force acting from the Sun to the spacecraft [67]

$$
\boldsymbol{F}_{d(S U N)}=\frac{\partial\left(U_{S U N}\right)}{\partial \boldsymbol{r}}
$$


Two approximations can be made to simplify Equation (2.32). The distance from the asteroid to the Sun is much larger than the distance from the spacecraft to the asteroid, therefore $\boldsymbol{r} / \boldsymbol{d} \ll 1$. In addition, $m_{\text {Spacecraft }} \ll m_{\text {Asteroid }}$. We applied these two facts which together are essentially Hill's approximation.

$$
\boldsymbol{F}_{d(S U N)}=-\frac{\mu_{s}}{2 d^{3}} \boldsymbol{r}
$$

The perturbation described above, the solar gravitational field, the solar radiation pressure, and the third body perturbations, are time-varying quantities. For spacecraft orbiting around a small celestial body, the irregular shaped asteroid's potential distribution is by far the most dominant parameters. Therefore, for the ease of numerical simulation, the 3 perturbations shown above are combined with any possible unmodelled plant dynamics and plant parameter variations to developed the following external disturbance model, $F_{d x}, F_{d y}$, and $F_{d z}$, presented in Equation (2.34) to (2.36).

$$
\begin{aligned}
& F_{d x}=K_{d s} * \sin (i * t) \\
& F_{d y}=K_{d s} * \cos (i * t) \\
& F_{d z}=K_{d s} * \sin (i * t)
\end{aligned}
$$

where $K_{d s}$ is the weight of the disturbance, and $i=1,2, \cdots, 6$ is multiplying to the time period of the disturbance $t$, to distinguish that each agent, $i$, is experiencing a different frequency of disturbance in the spacecraft formation system. $K_{d s}$ is said to be $3 \mathrm{mN}$ which is $60 \%$ of the maximum actuator force explained below. Figure 2.6 shows the external disturbance force that is acting on agent 1 . 


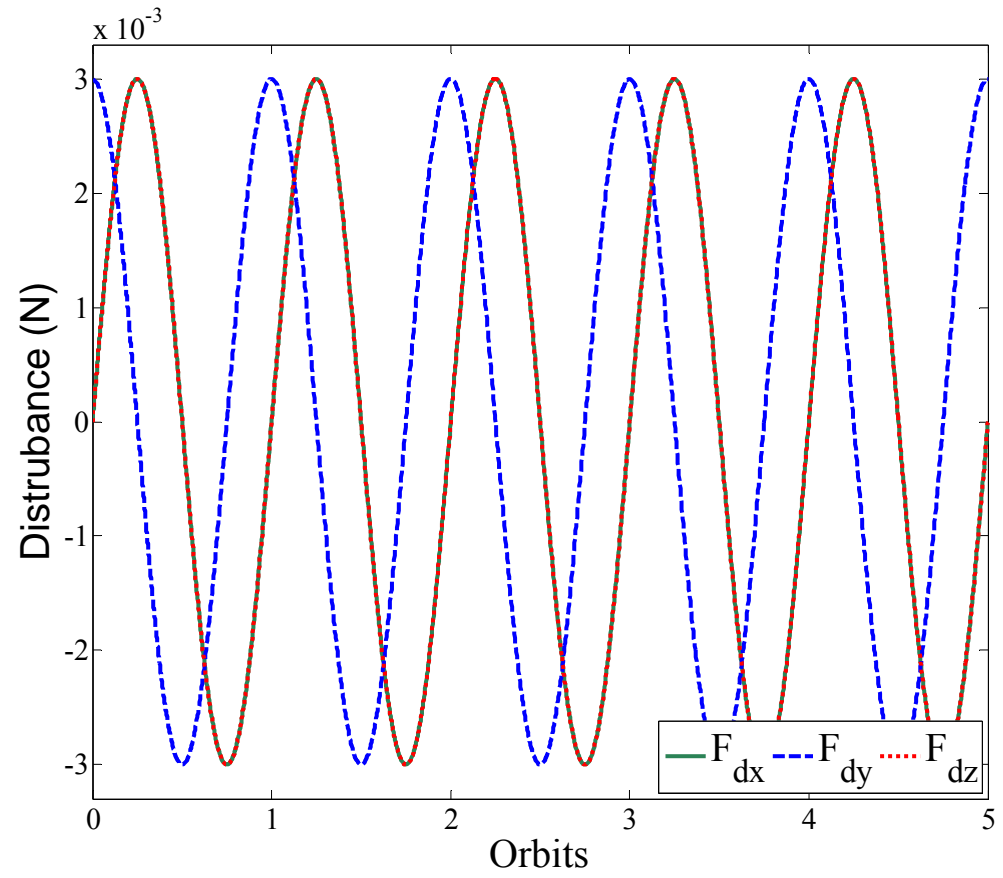

Figure 2.6 Disturbance force applied to agent 1 (i=1)

Adding the external disturbance and system control input, $\boldsymbol{u}_{f}$, to the equation of motion presented in Equation (2.19) to Equation (2.21) yields,

$$
\begin{gathered}
\ddot{x}-2 \varpi_{T} \dot{y}=\varpi_{T}^{2} x-\frac{\mu x}{r^{3}}+\frac{\partial U_{g 2}}{\partial x}+F_{d x}+u_{f x} \\
\ddot{y}+2 \varpi_{T} \dot{x}=\varpi_{T}^{2} y-\frac{\mu y}{r^{3}}+\frac{\partial U_{g 2}}{\partial y}+F_{d y}+u_{f y} \\
\ddot{z}=-\frac{\mu z}{r^{3}}+\frac{\partial U_{g 2}}{\partial z}+F_{d z}+u_{f z}
\end{gathered}
$$




\subsubsection{Minimum Control Input Required}

Minimum control input required is formulated by the difference between the reference trajectory, $\ddot{\boldsymbol{x}}_{c}$, and the system of desired state, $f\left(\boldsymbol{x}_{c}\right)$.

$$
\boldsymbol{u}_{d}=\ddot{\boldsymbol{x}}_{c}-f\left(\boldsymbol{x}_{c}\right)
$$

where $\ddot{\boldsymbol{x}}_{c}$ can be calculated by taking the derivative of Equation (2.26). The system of desired state, $f\left(\boldsymbol{x}_{c}\right)$, is calculated by substituting the desired states in Equation (2.37) to (2.39).

$$
\begin{gathered}
f\left(x_{c}\right)=2 \varpi_{T} \dot{y}_{c}+\varpi_{T}^{2} x_{c}-\frac{\mu x_{d}}{r_{c}{ }^{3}}+\frac{\partial U_{g 2}}{\partial x_{c}}+F_{d x}+u_{d x} \\
f\left(y_{c}\right)=-2 \varpi_{T} \dot{x_{c}}+\varpi_{T}^{2} y_{c}-\frac{\mu y}{r_{c}{ }^{3}}+\frac{\partial U_{g 2}}{\partial y_{c}}+F_{d y}+u_{d y} \\
f\left(z_{c}\right)=-\frac{\mu z_{c}}{r_{c}{ }^{3}}+\frac{\partial U_{g 2}}{\partial z_{c}}+F_{d z}+u_{d z}
\end{gathered}
$$

The minimum control input required is zero when there is no control required for tracking a reference trajectory. However, the proposed reference trajectory required small amount of control effort as shown in Figure 2.7 and Figure 2.8. $u_{d}$ is the minimum input force the system required to track the proposed reference trajectory. Figure 2.7 presents the profile of the required control input force when external disturbances are not applied to the system. When the proposed external disturbance model is applied, it becomes the dominating force in the system dynamics. Therefore, the minimum input required has increased to maintain system stability, and the results can be found in Figure 2.8. Noticed that line $u_{f x}$ is the same as $u_{f z}$ which is explained in Equation (2.34) and (2.36). 


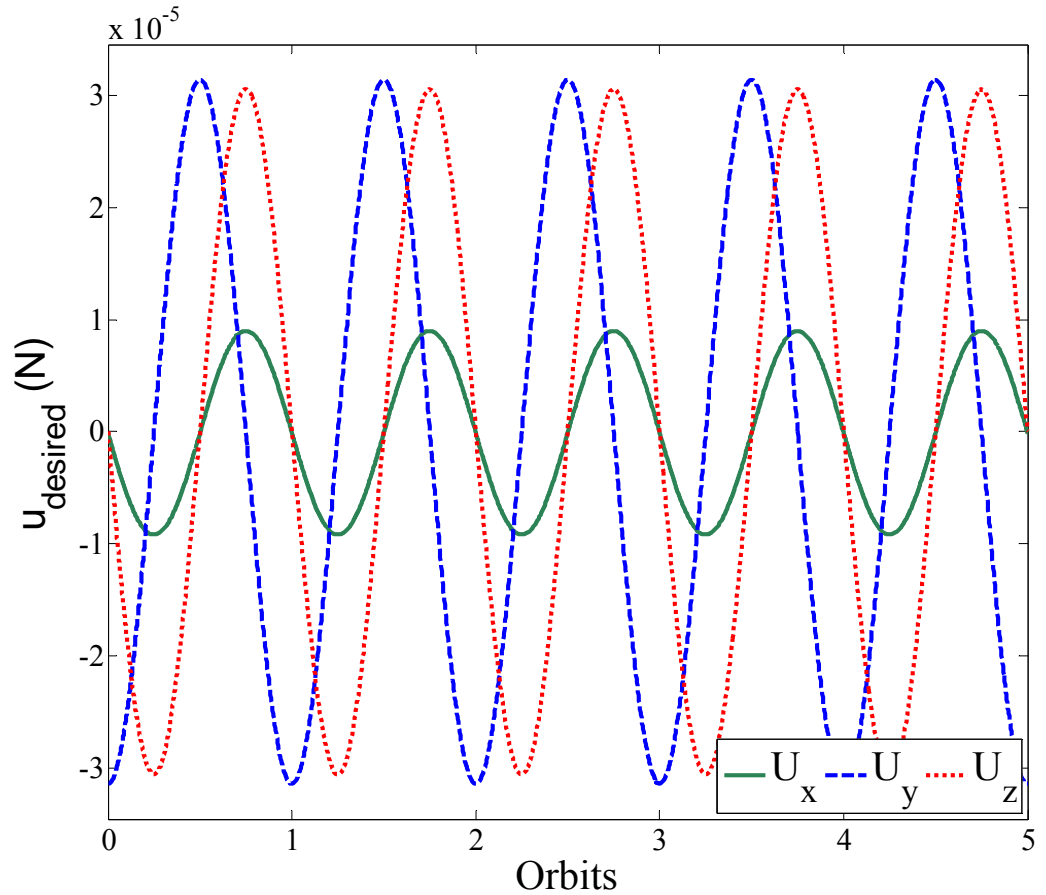

Figure 2.7 Minimum control input required (without disturbance)

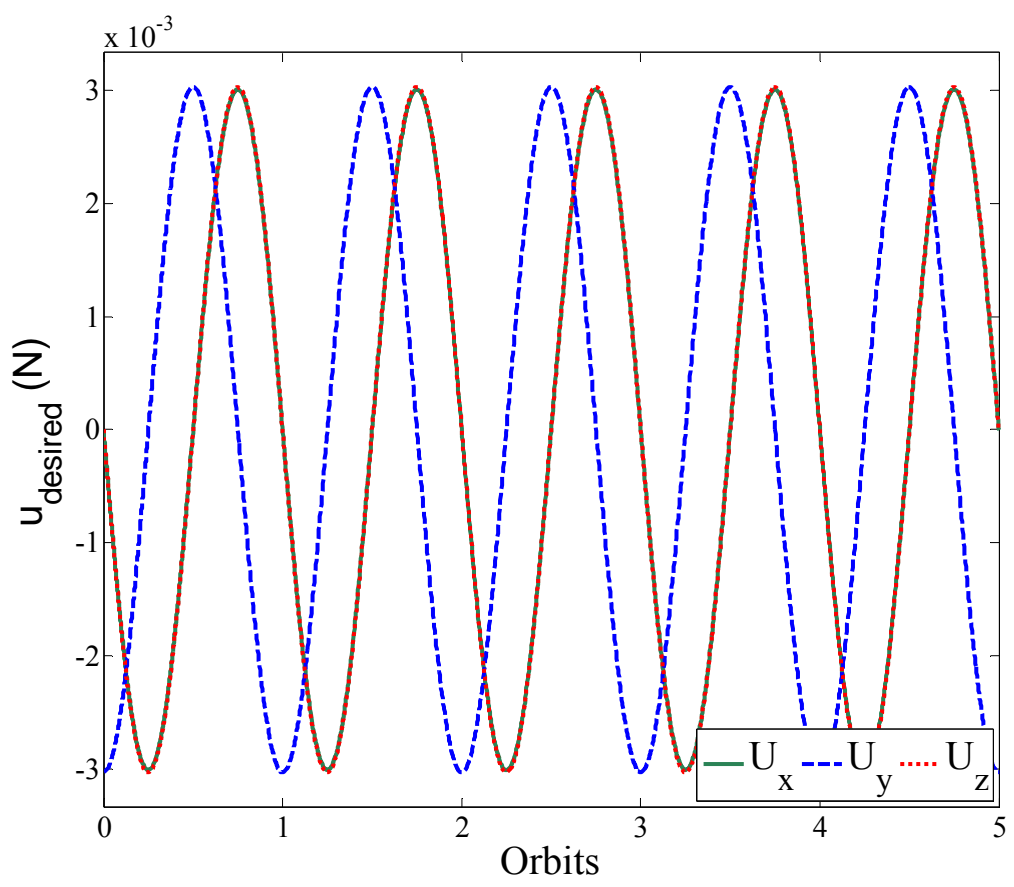

Figure 2.8 Minimum control input required for agent 1 (with disturbance) 


\subsection{Multi-AgENT System}

Fuel saving has been one of the main objectives in past space missions. It is usually achieved by reducing power consumption of a payload, designing a more efficient actuator, adjusting mission objectives, and/or the controller redesign. We often neglect another fundamental cost of the missions; the cost of the high performance and complex hardware. Rather than having a single monolithic orbiter or spacecraft to complete a mission objective, as is common in a multiagent system, we use multiple inexpensive, miniature spacecraft to achieve the same objective with more flexible procedure through coordination. Essentially, the goal is to replace demanding hardware with multiple copies of simple hardware.

The consensus problem, also known as the agreement problem, occurs when information or data converge to a common value. Consensus control can be separated into two categories; formation control problems or non-formation control problems. Nonetheless, cooperative control presents significant theoretical and practical challenges [68]. In order to have successful coordinate cooperation, three important issues must be tackled. The first, consensus seeking, commonly depends on the relationship between the graph topologies and the algebraic graph matrices. The second issue is formation keeping, and both centralized and decentralized approaches have been investigated in numerous studies. Last, trajectory tracking, has brought new interest to researchers because it imposes nonholonomic constraints to the system. Consensus algorithms assign tasks to multi-agents and only neighbour-to-neighbour communication between agents is provided. The information states of each agent are updated by the information states of their neighbour. The objective is to develop an update law so that information will be shared and converge to a common value.

\subsubsection{Graph Theory}

Graph theory is different than the graphing of algebraic functions or other kinds of graphs. Graph, $G(\mathbb{N}, \mathbb{E})$, in graph theory is a mathematical way to represent the connection between subjects, and these subjects are call nodes, $\mathbb{N}=(1, \ldots, n)$, or vertices. Edge, $\mathbb{E}$, is the connection between two vertices. These edges can be directional or non-directional. Graph theory is gaining popularity because of the advance in computer science, as well as the use of communication networks. These communication network graphs can be interpreted and applied to many situations. For example, if graph theory is applied to map human social interactions, then it can be used to 
define the relationship of a family, friendship among friends, or human resources' collaboration within an organization. In a more engineering problem point of view, if it is applied to a mobile network, it will represent the signal and data transfer between the base station, ground station or portable transceiver, and each wireless phone or other electronic device on the network. Every edge is connected with two nodes, $(i, j)$, and $\mathbb{E}(i, j)$ denotes the information flow from $i$ to $j$, where $i$ and $j$ are commonly called the parent node and the child node, respectively. However, there is a specific case where the node acts as a parent node and a child node. This is called self-edge, and it is identified as $\mathbb{E}(i, i)$. For a directed graph, the signal transfers from parent node to child node. An undirected edge is expressed as a distinctive scenario of a pair of directed edges such that $\mathbb{E}(i, j)=\mathbb{E}(j, i)$. In the mobile network example, the interaction between each "node" is two-way, which implies that the signal is transferred back and forth in two directions.

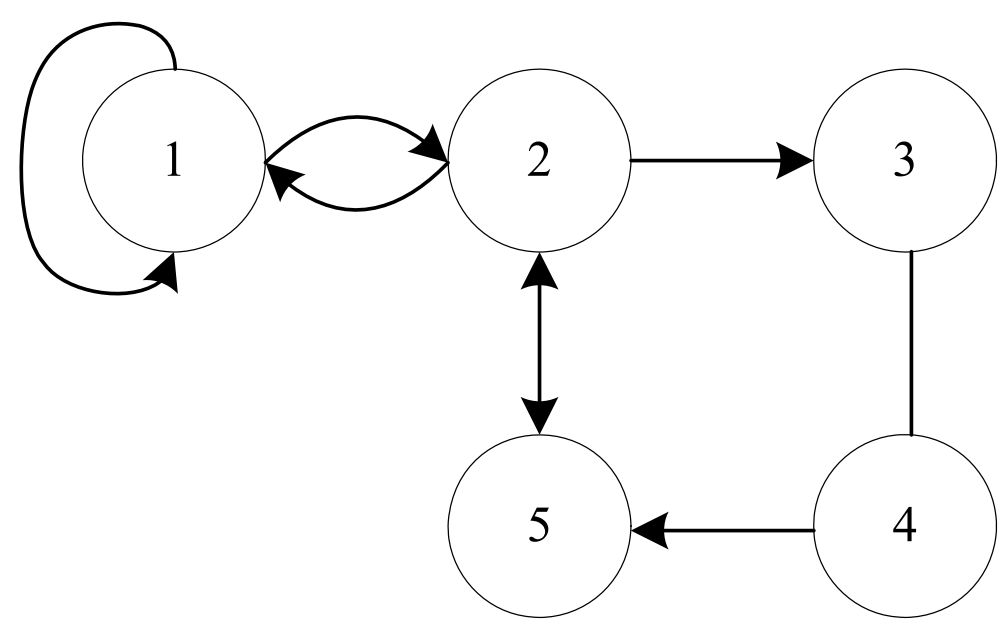

Figure 2.9 Communication graph among five spacecraft

Graph theory can also be applied to space mission formation flying, to map out the communication links between each spacecraft. We can take Figure 2.9 as an example of a communication map of a 5 spacecraft formation flying system. All edges are directed edge in this system except one self-edge, $\mathbb{E}(1,1)$. However, we can identify three pairs of directed edges as undirected edges, which are, $\{\mathbb{E}(1,2), \mathbb{E}(2,5), \mathbb{E}(3,4)\}$. An undirected edge is usually shown in a line without arrows, i.e. $\mathbb{E}(3,4)$, however, it can also be drawn with two directed edges, i.e. $\mathbb{E}(1,2)$, or one line that ends with two arrows, i.e. $\mathbb{E}(2,5)$, in order to avoid misunderstanding whether the arrow is missed or not. 


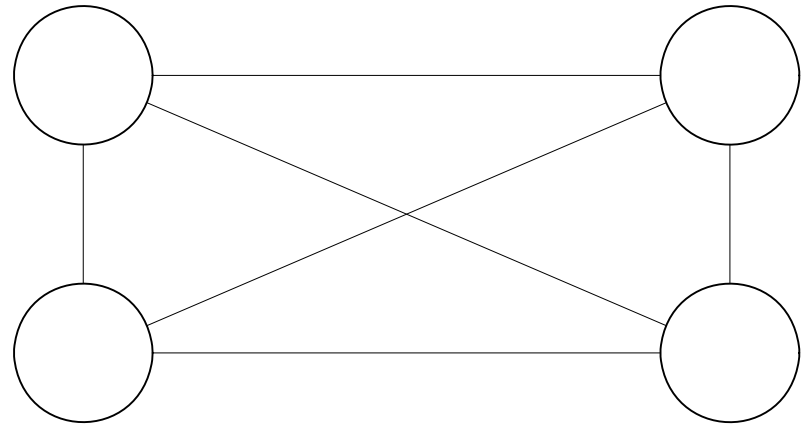

( a )

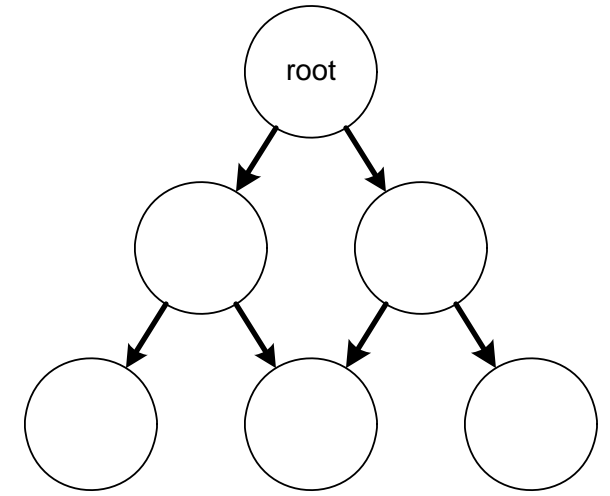

(b)

Figure 2.10 (a) Strongly connected undirected graph, (b) rooted directed tree

A path is a chain of edges, and can also be a subset of a graph. If a directed path starts and ends at the same node, it is called a cycle. A strongly connected path is one where every node is connected to each other with an edge, as shown in Figure 2.10 (a). In contrast, a rooted directed tree is a directed graph in which there is one node that has no parent node, the root, and all the other nodes are connected to only one parent node, shown in Figure 2.10 (b).

\subsubsection{Properties of Matrices in Graph Theory}

The adjacency matrix, $\mathcal{A}=\left[a_{i j}\right]$, is a $n \times n$ weight matrix that represents the connectivity between each node. $n$ is the number of agents in the consensus network, $i=1,2, \ldots n$ and $j=$ $1,2, \ldots n$. Notice that all communication links, the edges $(\mathbb{E})$ in the graph, are weighted; $a_{i j}$ will be assigned a positive weight value if $\mathbb{E}(j, i)$ exists. If the communication strength is irrelevant for the application, then $a_{i j}$ is set to 1 for all $\mathbb{E}(j, i)$. Self-edges are allowed, where $a_{i i}>0$. Equation (2.44) is the adjacency matrix for Figure 2.9. $a_{11}=1$ is the self-edge; $a_{12}=a_{21}=0.9, a_{25}=$ $a_{52}=0.8$, and $a_{34}=a_{43}=0.7$ are the undirected edges; The directed edge flow from node 2 to 3 and node 4 to 5 are, $a_{32}=0.6$ and $a_{54}=0.5$, respectively.

$$
\mathcal{A}=\left[\begin{array}{ccccc}
1 & 0.9 & 0 & 0 & 0 \\
0.9 & 0 & 0 & 0 & 0.8 \\
0 & 0.6 & 0 & 0.7 & 0 \\
0 & 0 & 0.7 & 0 & 0 \\
0 & 0.8 & 0 & 0.5 & 0
\end{array}\right]
$$


For all undirected graphs, $\mathcal{A}$ is symmetric. The matrix is said to be balanced when $\sum_{j=1}^{n} a_{i j}=$ $\sum_{j=1}^{n} a_{j i}$, hence $\mathcal{A}$ for an undirected graph is also balanced.

The degree matrix, $\mathcal{D}$, is a diagonal matrix which will also be weighted in the same manner as the adjacency matrix. $\mathcal{D}=\left[d_{i j}\right] \in \mathbb{R}^{n \times n}$ is a graph with node set $\mathbb{N}=(1, \ldots, n)$ and it is defined such that $d_{i i}=\sum_{j}^{n} a_{i j}$. Equation (2.45) is the degree matrix for the graph in Figure 2.9. If all decimal places are rounded up or the entries in $\mathcal{A}$ are not weighted, then the integer numerical value in the approximate degree matrix corresponds to the number of incoming edges of each node.

$$
\mathcal{D}=\left[\begin{array}{ccccc}
1.9 & 0 & 0 & 0 & 0 \\
0 & 1.7 & 0 & 0 & 0 \\
0 & 0 & 1.3 & 0 & 0 \\
0 & 0 & 0 & 0.7 & 0 \\
0 & 0 & 0 & 0 & 1.3
\end{array}\right] \approx\left[\begin{array}{ccccc}
2 & 0 & 0 & 0 & 0 \\
0 & 2 & 0 & 0 & 0 \\
0 & 0 & 2 & 0 & 0 \\
0 & 0 & 0 & 1 & 0 \\
0 & 0 & 0 & 0 & 2
\end{array}\right]
$$

The Laplacian matrix $\mathcal{L}=\left[\ell_{i j}\right] \in \mathbb{R}^{n \times n}$ is the difference between the Degree matrix and the Adjacency matrix, $\mathcal{L}=\mathcal{D}-\mathcal{A}$. It can also be defined as $\ell_{i j}=-a_{i j}$ with the condition of $\ell_{i i}=$ $\sum_{j \neq i} a_{i j}$. In summary, the properties of a Laplacian matrix are, $\ell_{i j} \leq 0$ for $i \neq j$, and the sum of each row in the Laplacian matrix is zero, $\sum_{j=1}^{n} \ell_{i j}=0$ for $i=1,2, \ldots n$.

$$
\mathcal{L}=\left[\begin{array}{ccccc}
0.9 & -0.9 & 0 & 0 & 0 \\
-0.9 & 1.7 & 0 & 0 & -0.8 \\
0 & -0.6 & 1.3 & -0.7 & 0 \\
0 & 0 & -0.7 & 0.7 & 0 \\
0 & -0.8 & 0 & -0.5 & 1.3
\end{array}\right]
$$

Equation (2.46) is the Laplacian matrix for the graph in Figure 2.9. The subset of the undirected node pairs in the graph is reflected by the symmetric portions of the matrix. Zero is an eigenvalue of $\mathcal{L}$ because of the zero row sum, and the eigenvector will be a $n \times 1$ column vector of ones. $\mathcal{L}$ is diagonally dominant, and all values are positive. Following the Gershgorin's disc theorem [44], all nonzero eigenvalues of $\mathcal{L}$ for an undirected graph are positive, and all nonzero eigenvalues of $\mathcal{L}$ for a directed graph have positive real parts. Consequently, all nonzero eigenvalues of $-\mathcal{L}$ for both directed and undirected graphs will have negative real parts. 


\subsubsection{Consensus Algorithm}

The objective of a consensus algorithm is to execute similar controls on the states of each agent in the network. If the information is transferred continuously in the communication process, the differential equation is used to model the algorithm, otherwise, the difference equation is used if data arrive in a discrete signal pack. A first order differential equation is used to showcase the information state transfer using a consensus algorithm in this section. The decentralized formation flying communication topology can be mapped out with the graph theory proposed above, and the connection and interaction path is modeled mathematically with the adjacency matrix. A conventional continuous consensus algorithm [44] is

$$
\dot{\varrho}_{i}(t)=-\sum_{j=1}^{n} a_{i j}(t)\left(\varrho_{i}(t)-\varrho_{j}(t)\right) \quad \begin{aligned}
& i=1,2, \cdots, n \\
& j=1,2, \cdots, m
\end{aligned}
$$

Notice that the communication topology, the adjacency matrix $a_{i j}(t)$, may or may not necessarily be time invariant. It can vary with time due to vehicle motion or communication dropouts, or it can be constant by assuming perfect condition. Perfect condition is applied for this research. $\varrho_{i}$ and $\varrho_{j}$ is the information state of the $i$ th and $j$ th agent, respectively. $n=m$ is the number of agent in the system. The result of this topology is that the value of state $x_{i}$ depends on the state value of its neighbours.

\subsubsection{Decentralized Formation Flying Mathematical Model}

Consider a multi-agent system, including $n$ agents, in which the $i$ th agent is described by the following second-order differential equation

$$
\begin{gathered}
\dot{\boldsymbol{x}}_{i}=\boldsymbol{v}_{i} \\
\dot{\boldsymbol{v}}_{\boldsymbol{l}}=f_{i}\left(\boldsymbol{x}_{i}, \boldsymbol{v}_{i}\right)+g_{i}\left(\boldsymbol{x}_{i}\right) u_{i}+\vartheta_{i}, \quad i=1,2, \cdots, n
\end{gathered}
$$

where $\boldsymbol{x}_{i} \in \mathbb{R}^{3}=\left[\begin{array}{lll}x & y & z\end{array}\right]^{T}$ and $\boldsymbol{v}_{i} \in \mathbb{R}^{3}=\left[\begin{array}{lll}\dot{x} & \dot{y} & \dot{z}\end{array}\right]^{T}$ are the state vectors of the $i$ th agent, respectively; $f_{i}\left(x_{i}, v_{i}\right) \in \mathbb{R}^{3 \times 3}$ is a vector function that corresponds to the relative equations of 
motion, $g_{i}\left(x_{i}\right) \in \mathbb{R}^{3 \times 3}$ represents the input matrix control gain and is assumed to be non-singular. $u_{i} \in \mathbb{R}^{3}=\left[\begin{array}{lll}u_{f x} & u_{f y} & u_{f z}\end{array}\right]_{i}{ }^{T}$ denotes the control input vector of the $i$ th agent, and $\vartheta_{i} \in \mathbb{R}^{3}=$ $\left[\begin{array}{lll}F_{d x} & F_{d y} & F_{d z}\end{array}\right]_{i}^{T}$ is the bounded external disturbance explained in Chapter 2.1.5.

The disturbances $\vartheta_{i}(i=1,2, \cdots, n)$ are bounded such that

$$
\left\|\vartheta_{i}\right\| \leq \vartheta_{M i}
$$

where $\vartheta_{M i}$ is a positive constant. In the reference trajectory defined in Chapter 2, its first and second derivatives are considered to be in a compact set as follows

$$
\Omega_{d} \equiv\left\{\left(x_{d}, \dot{x}_{d}, \ddot{x}_{d}\right):\left\|x_{d}\right\|^{2}+\left\|\dot{x}_{d}\right\|^{2}+\left\|\ddot{x}_{d}\right\|^{2} \leq c_{1}\right\}
$$

where $c_{1}$ is a positive constant. This assumption is commonly used in the literature [55] on consensus tracking control for second-order multi-agent systems.

For clarification and ease of explanation in the following chapter, a specified scenario of the graph theory is brought from the previous chapter, and any background information may refer to Chapter 2.2.1. The topology of the information flow between agents is described by a weighted graph $G=(\Upsilon, E, A)$, where $\Upsilon=\left(r_{1}, r_{2}, \cdots r_{n}\right)$ is the set of nodes; $E \subseteq \Upsilon \times \Upsilon$ is the set of edges; and $A=\left(a_{i j}\right) \in \mathbb{R}^{n \times n}$ is the weighted adjacency matrix of graph $\mathrm{G}$ with non-negative elements, where $a_{i i}=0$ and $a_{i j} \geq 0$. Node $r_{i}(i=1,2, \cdots, n)$ represents the $i$ th agent, and an edge in $G$ is denoted by an unordered pair $(i, j) .(i, j) \in E$ if and only if there is the information exchange between the $i$ th agent and the $j$ th agent, that is, $a_{i j}=a_{j i}$. The adjacency element $a_{i j}$ denotes the communication quality between the $i$ th agent and the $j$ th agent, that is, $(i, j) \in E=a_{i j}>0$. A graph is simple if it has no self-edge or repeated edges. For this dissertation, an undirected graph is applied, and it is assumed that the graph $G$ is simple. Let $D=\operatorname{diag}\left\{d_{1}, d_{2}, \cdots d_{n}\right\}$ be the degree matrix of $G$, whose diagonal elements are give by $d_{i}=\sum_{j=1}^{n} a_{i j}(i=1,2, \cdots, n)$. Then the Laplacian matrix $L$, a symmetric matrix, of the weighted graph $G$ is defined by

$$
L=D-A
$$




\section{System Error Model}

Two state errors are considered for each agent in consensus tracking control for multi-agent systems, these being, absolute and relative state errors. The state error of an individual agent with respect to the reference trajectory is the absolute error, which this state error of the $i$ th follower agent are shown below

$$
\begin{gathered}
e_{x i}=\boldsymbol{x}_{i}-\left(\boldsymbol{x}_{c}+\boldsymbol{h}_{i}\right) \\
e_{v i}=\boldsymbol{v}_{i}-\dot{\boldsymbol{x}}_{d}
\end{gathered}
$$

where $\boldsymbol{h}_{i}$ is the distance between the trajectory $\boldsymbol{x}_{c}$, defined in Equation (2.26), and the $i$ th agent in the formation. $\boldsymbol{x}_{d}=\left(\boldsymbol{x}_{c}+\boldsymbol{h}_{i}\right)$, which will be the reference trajectory for each agent. In this study, $\boldsymbol{h}_{i}$ is time invariant, therefore, $\dot{\boldsymbol{h}}_{i}=0$.

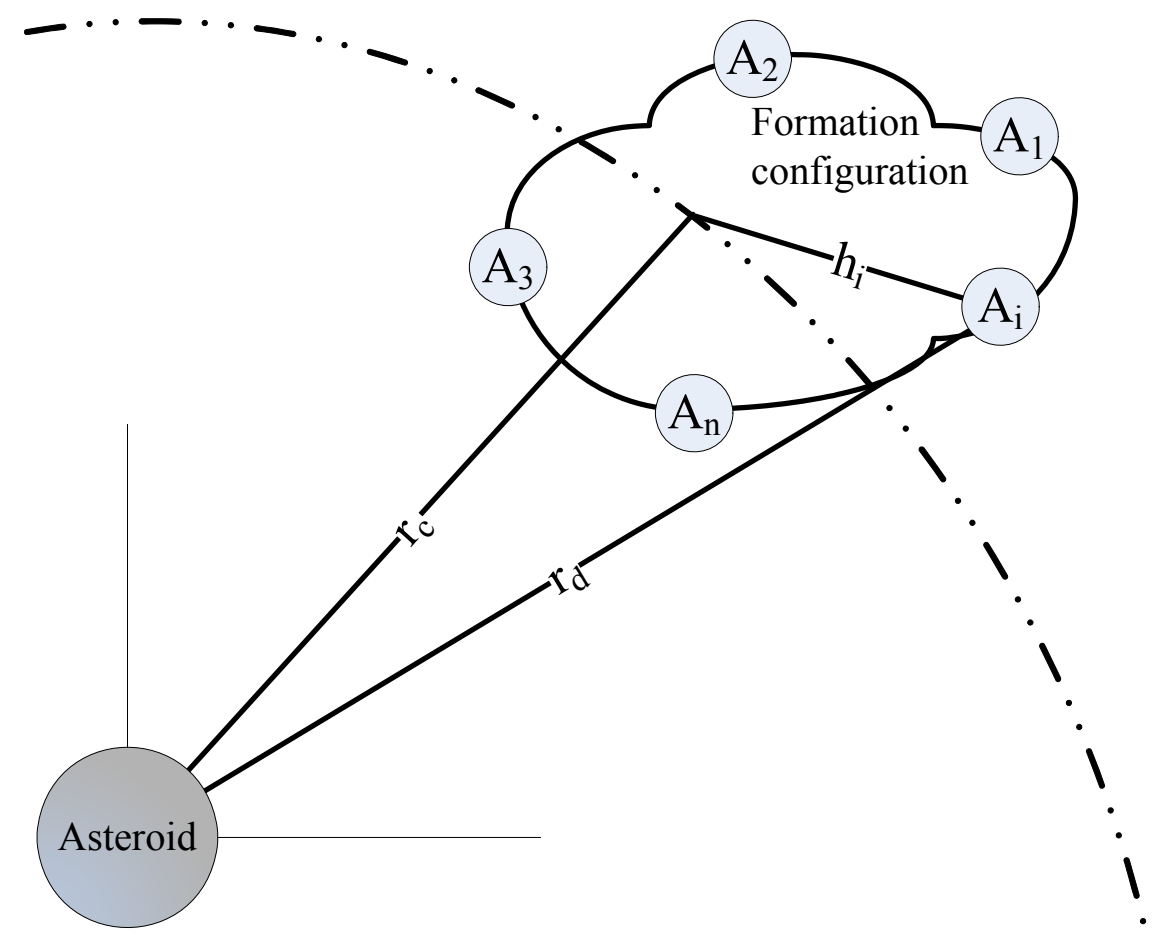

Figure 2.11 Reference trajectory for each agent

Appling Equation (2.48) and (2.49), the dynamic equations for the absolute errors $e_{x i}$ and $e_{v i}$ $(i=1,2, \cdots, n)$ becomes 


$$
\begin{gathered}
\dot{e}_{x i}=e_{v i} \\
\dot{e}_{v i}=f_{i}\left(\boldsymbol{x}_{i}, \boldsymbol{v}_{i}\right)+g_{i}\left(\boldsymbol{x}_{i}\right) u_{i}+\vartheta_{i}-\ddot{\boldsymbol{x}}_{d}
\end{gathered}
$$

and the compact form is rewritten in Equation (2.57) and (2.58)

$$
\begin{gathered}
\dot{e}_{x}=e_{v} \\
\dot{e}_{v}=\mathrm{F}+\mathrm{g}(\boldsymbol{x}) \mathrm{u}+\vartheta-X_{d}
\end{gathered}
$$

where the absolute errors $e_{x}=\left(e_{x 1}{ }^{T}, e_{x 2}{ }^{T}, \cdots e_{x n}{ }^{T}\right)^{T} \in R^{3 n}, e_{v}=\left(e_{v 1}{ }^{T}, e_{v 2}{ }^{T}, \cdots e_{v n}{ }^{T}\right)^{T} \in R^{3 n}$, the dynamic system $F=\left(f_{1}{ }^{T}, f_{2}{ }^{T}, \cdots f_{n}{ }^{T}\right)^{T} \in R^{3 n}$, control gain matrix $g(\boldsymbol{x})=$ $\operatorname{diag}\left\{g_{1}, g_{2}, \cdots g_{n}\right\} \in R^{3 n \times 3 n} \quad$ with $\quad \boldsymbol{x}=\left(\boldsymbol{x}_{1}{ }^{T}, \boldsymbol{x}_{2}{ }^{T}, \cdots \boldsymbol{x}_{n}{ }^{T}\right)^{T} \in R^{3 n}$, control input $u=$ $\left(u_{1}{ }^{T}, u_{2}{ }^{T}, \cdots u_{n}{ }^{T}\right)^{T} \in R^{3 n}$, disturbance $\vartheta=\left(\vartheta_{1}{ }^{T}, \vartheta_{2}{ }^{T}, \cdots \vartheta_{n}{ }^{T}\right)^{T} \in R^{3 n}$, and the desired trajectory $X_{d}=\left(\ddot{x}_{d}^{T}, \ddot{x}_{d}^{T}, \cdots \ddot{x}_{d}^{T}\right)^{T} \in R^{3 n}$.

In multi-agent system, the relative error is the state error of an individual agent with respect to the other. The following equations shows the relative state errors between the $i$ th and $j$ th agents

$$
\begin{aligned}
& r_{x i j}=e_{x i}-e_{x j} \\
& r_{v i j}=e_{v i}-e_{v j}
\end{aligned}
$$

where $i=j=1,2, \cdots n$.

In a mission, the common desired trajectory is only available to a subset of agents, and each agent will have limited access to other agents in the group. Lumped state errors ( $\alpha_{x i}$ and $\alpha_{x i}$ ) that include the absolute and relative state errors are necessary to measure the true tracking errors of the whole system. The $i$ th agent may not obtain the absolute state errors $\left(e_{x i}\right.$ and $e_{v i}$ $(i=1,2, \cdots, n))$ and all relative state errors $\left(r_{x i j}\right.$ and $\left.r_{v i j}(i=j=1,2, \cdots, n)\right)$. With the consideration of the above facts, using the weighted adjacency matrices $A$, we define lumped state errors as 


$$
\begin{aligned}
& \alpha_{x i}=\sum_{j=1}^{n} a_{i j} r_{x i j}+k_{i} e_{x i} \\
& \alpha_{v i}=\sum_{j=1}^{n} a_{i j} r_{v i j}+k_{i} e_{v i}
\end{aligned}
$$

where $\alpha_{x i}$ and $\alpha_{v i} \in R^{3}(i=1,2, \cdots, n) . a_{i j}$ is the element of the weighted adjacency matrix $A$ and $k_{i}$ is the element of the weighted matrix K. The lumped state errors are the sum of the absolute and relative state errors and only depend on the information of the neighbour agents of the $i$ th agent. The controller for each agent is developed based on the lumped state errors $\alpha_{x i}$ and $\alpha_{v i} \in$ $R^{3}$ where $(i=1,2, \cdots, n)$.

With the purpose of aiding the following theoretical analysis, the lumped state errors $\alpha_{x i}$ and $\alpha_{v i} \in R^{3}$ where $(i=1,2, \cdots, n)$ can be rewritten in the function of absolute state errors $\left(e_{x i}\right.$ and $e_{v i}$ ) through simple algebraic transformation, with the use of the graph Laplacian matrix L, as follows

$$
\begin{aligned}
& \alpha_{x i}=\sum_{j=1}^{n} a_{i j} r_{x i j}+K e_{x i}=\sum_{j=1}^{n} l_{i j} e_{x j}+K e_{x i} \\
& \alpha_{v i}=\sum_{j=1}^{n} a_{i j} r_{v i j}+K e_{v i}=\sum_{j=1}^{n} l_{i j} e_{v j}+K e_{v i}
\end{aligned}
$$

The lumped state errors in Equations (2.63) and (2.64) can be defined as $\alpha_{x}=$ $\left(\alpha_{x 1}{ }^{T}, \alpha_{x 2}{ }^{T}, \cdots \alpha_{x n}{ }^{T}\right)^{T} \in R^{3 n}$ and $\alpha_{v}=\left(\alpha_{v 1}{ }^{T}, \alpha_{v 2}{ }^{T}, \cdots \alpha_{v n}{ }^{T}\right)^{T} \in R^{3 n}$, respectively. Re-stated in terms of $M,[55]$

$$
\begin{aligned}
& \alpha_{x}=M e_{x} \\
& \alpha_{v}=M e_{v}
\end{aligned}
$$


where $M=(L+K) \otimes I_{3}$ and Kronecker product is shown in $\otimes$, which assures that $M$ is a symmetric and positive-definite matrix. Using Equations (2.57) and (2.58), the dynamic equations for $\alpha_{x}$ and $\alpha_{v}$ is shown below

$$
\begin{gathered}
\dot{\alpha}_{x}=\alpha_{v} \\
M^{-1} \dot{\alpha}_{v}=\mathrm{F}+g \mathrm{u}+\vartheta-X_{d}
\end{gathered}
$$

Equations (2.67) and (2.68) will be used in the subsequent chapters when studying the stabilities of the proposed control law.

The system model and multi-agent system shown in this chapter will be used in the following chapters to develop control laws that stabilise the system plant and satisfy certain performance levels in the presence of external disturbance force, sensor signal interferences, uncertain plant dynamics and system parameter variation. Sliding mode control is the first control technique applied to the multi-agent system orbiting around asteroid and will be studied in the next chapter. 


\section{SLIDING MODE CONTROL OF CONSENSUS SPACECRAFT FORMATION FLYING}

Consensus spacecraft formation flying is dependent on system state information transfer between spacecraft (agents). Constructing an efficient multi-agent network and formulating an appropriate consensus algorithm is the top priority in controlling spacecraft's decentralized formation. In this chapter, the proposed control law applies the consensus algorithm defined in Chapter 2.2.3, in order to control multiple spacecraft to fly collaboratively. Lyapunov theory is used to prove the stability for both the reaching phase and the sliding phase of the variable structure control. The nonlinear control algorithm based on the sliding mode technique is integrated with the consensus algorithm and formulated with a detailed proof of stability for the closed-loop system. Furthermore, the results of numerical simulations incorporating different sensor fault scenarios are presented for a detailed assessment of the system performance under the proposed control strategies and validation of the established theoretical framework. Finally, some brief conclusions are provided.

\subsection{DESIGN OF CONTROL LAWS}

In this section, a control law is developed for the decentralized formation flying system. The goal is that this nonlinear controller will drive the state trajectory of the nonlinear system onto a sliding or switching surface. Sliding mode control is a nonlinear control method. It alters the dynamics of a nonlinear system through the application of a discontinuous control signal that forces the system to "slide" along a cross-section of the system's normal behaviour. Sliding mode control is an example of variable structure control (VSC), where it can switch from one continuous structure to another based on the current position in the state space. The multiple control structures allow the trajectories to slide along the boundaries of the control structures. The sliding surface is the geometrical locus consisting of the boundaries. The property of remaining on the switching surface once intercepted is called a sliding mode, where the behaviour of the system is dominated by the lower-order dynamics and is inherently insensitive to external disturbances and model uncertainties. 


\subsubsection{Variable Structure Control}

Variable structure control (VSC) is a form of discontinuous nonlinear control. VSC system is a class of system where the 'control law' is intentionally changed during the control process. The control law changes based on predefined rules which depend on the state of the system. The application of a high-frequency switching control is used as a method of altering the dynamics of a nonlinear system. The state-feedback control law switches from one smooth condition to another, thus, it is not a continuous function of time. Therefore, the structure of the control law varies based on the position of the state trajectory. For the purpose of illustration, consider the following linear time-invariant system (adapted from [64]):

$$
\left\{\begin{array}{l}
\dot{\mathrm{x}}_{1}=x_{2} \\
\dot{\mathrm{x}}_{2}=u
\end{array}\right.
$$

In state-space form

$$
\dot{X}=A X+B U
$$

with $X \in \mathbb{R}^{2}=\left[\begin{array}{ll}x_{1} & x_{2}\end{array}\right]^{T}$, and $\mathrm{U}$ as a scalar control input. Matrices $A$ and $B$ are then expressed as

$$
A=\left[\begin{array}{ll}
0 & 1 \\
0 & 0
\end{array}\right], B=\left[\begin{array}{l}
0 \\
1
\end{array}\right]
$$

Next, a linear sliding surface is defined as

$$
S=K_{2} x_{2}+K_{1} x_{1}
$$

$K_{1}$ and $K_{2}$ are selected to be positive real numbers. Consider the VSC with a control law of 


$$
U=-K \operatorname{sgn}(S)=\left\{\begin{array}{cl}
-K & \text { if } S>0 \\
0 & \text { if } S=0 \\
K & \text { if } S<0
\end{array}\right.
$$

where $\mathrm{K}$ is a positive real scalar number that has a direct effect on the rate at which the sliding surface can be reached. The sliding condition defined in Equation (3.5) is used to regulate the system described by Equation (3.1). The phase portrait of the closed-loop system obtained for different initial conditions from using the control law given by Equation (3.5), with $K_{1}=K_{2}=1$ and $K=2$, is shown in Figure 3.. The set of points for which $S=0$ is denoted by the inclined line in Figure 3.. This line divides the phase plane into four regions given by

$$
\begin{array}{rrr}
\text { I: } \mathrm{x}_{1}>0, S>0 & \text { and } & \text { II: } \mathrm{x}_{1}>0, S<0 \\
\text { III: } \mathrm{x}_{1}<0, S<0 & \text { and } & \text { IV: } \mathrm{x}_{1}<0, S>0
\end{array}
$$

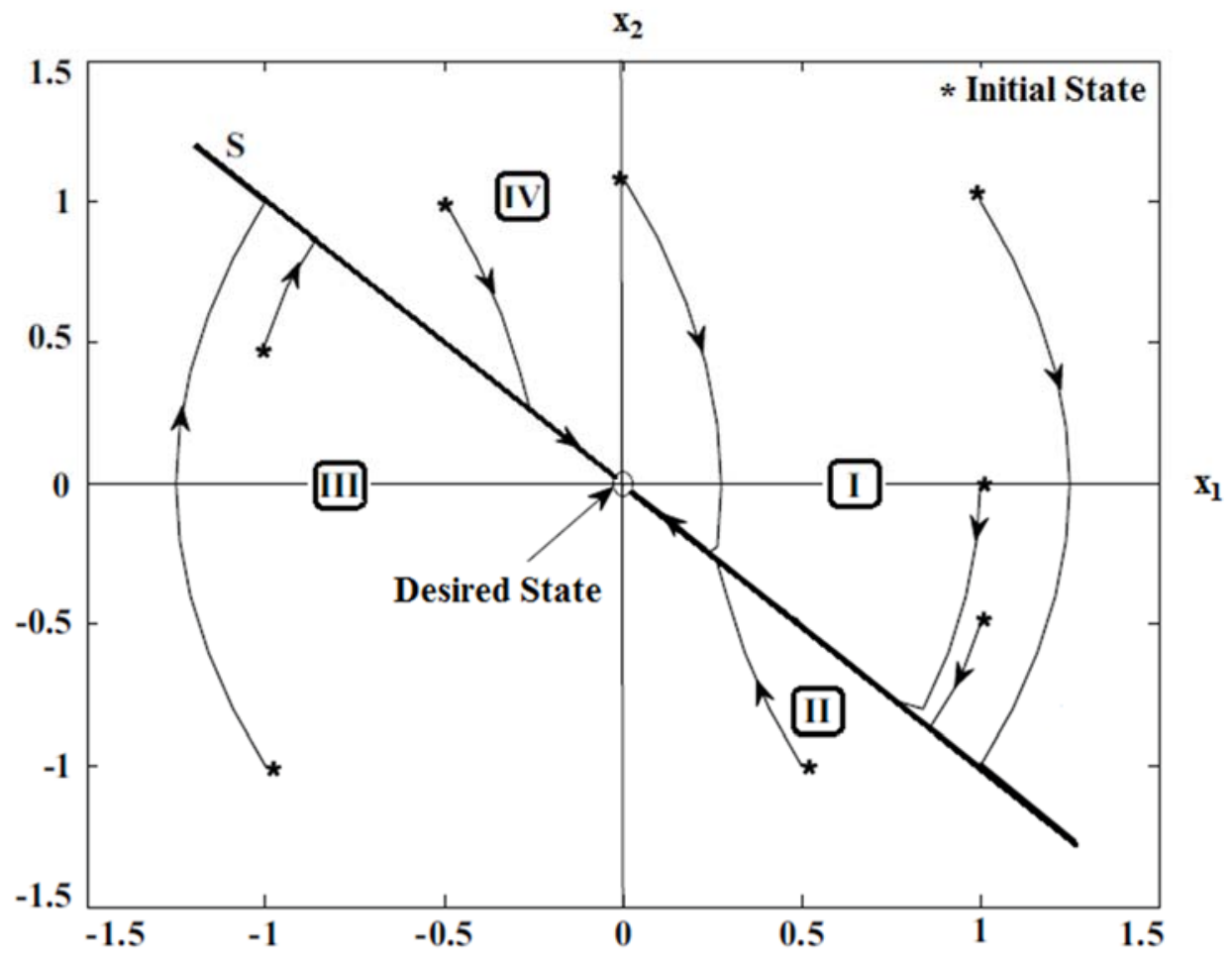

Figure 3.1: Phase portrait of the double integrator under VSC 
By choosing $V=S^{2} / 2$ as the Lyapunov function candidate, the reachability condition [64], $\dot{V}<0$, gives

$$
\begin{aligned}
\dot{V}=S \dot{S} & =\mathrm{S}\left(K_{2} \dot{\mathrm{x}}_{2}+K_{1} \dot{\mathrm{x}}_{1}\right) \\
& =\mathrm{S}\left(K_{2} \mathrm{u}+K_{1} x_{2}\right) \\
& =\mathrm{S}\left(K_{2}(-K \operatorname{sgn}(S))+K_{1} x_{2}\right) \\
& =(S * \operatorname{sgn}(S))\left(-\left(K_{2} * K\right)+K_{1} \frac{x_{2}}{\operatorname{sgn}(S)}\right) \\
& \leq|S|\left(-\left(K_{2} * K\right)+K_{1}\left|x_{2}\right|\right)<0
\end{aligned}
$$

Therefore,

$$
\lim _{S \rightarrow 0+} \dot{S}<0 \text { and } \lim _{S \rightarrow 0-} \dot{S}>0
$$

when $K_{1}\left|x_{2}\right|<\left(K_{2} * K\right)$. As a result, with the condition $K_{1}\left|x_{2}\right|<\left(K_{2} * K\right)$, the system reference path on either side of the line $S=0$ converges towards the sliding surface $S$. In the process of the sliding motion, lower-order dynamics dominate the behaviour of the system which is independent of the control. The choice of the parameters in the sliding surface, Equation (3.4), will highly affect the dynamic performance of the system. Furthermore, the control output only safeguards that the sliding surface is achieved and Equation (3.8) is satisfied.

Sliding mode control (SMC) is one of the commonly used technique in VSC. The strengths of SMC include low sensitivity to plant parameter uncertainty, greatly reduced order modeling of plant dynamics, and finite-time convergence due to discontinuous control law.

\subsubsection{Control Problem}

The proposed control law is designed based on a generalized framework. The equation of motion, Equation (3.9) - (3.11), defines the dynamics of a single spacecraft orbiting around an asteroid. See,

$$
\ddot{x}-2 \varpi_{T} \dot{y}=\varpi_{T}^{2} x-\frac{\mu x}{r^{3}}+\frac{\partial U_{g 2}}{\partial x}+u_{f x}
$$




$$
\begin{gathered}
\ddot{y}+2 \varpi_{T} \dot{x}=\varpi_{T}^{2} y-\frac{\mu y}{r^{3}}+\frac{\partial U_{g 2}}{\partial y}+u_{f y} \\
\ddot{z}=-\frac{\mu z}{r^{3}}+\frac{\partial U_{g 2}}{\partial z}+u_{f z}
\end{gathered}
$$

where

$$
U_{g 2}=-\frac{\mu C_{20}\left(x^{2}+y^{2}-2 z^{2}\right)}{2 r^{5}}+\frac{3 \mu C_{22}\left(x^{2}-y^{2}\right)}{r^{5}}
$$

To simplify the control design procedure, we re-formulate the relative dynamics model in a state-dependent parameterized form as follows,

$$
\dot{X}=A X+\mathrm{F}_{g}(X)+B U
$$

where $X \in \mathbb{R}^{6}=\left[\begin{array}{llllll}x & y & z & \dot{x} & \dot{y} & \dot{z}\end{array}\right]^{T}$ is the state vector, $A \in \mathbb{R}^{6 \times 6}$ is the linear component of the relative equations of motion, $F_{g} \in \mathbb{R}^{6}$ represents the lumped nonlinearities, $B \in \mathbb{R}^{6 \times 3}$ is the input matrix, and $U \in \mathbb{R}^{3}=\left[\begin{array}{lll}u_{f x} & u_{f y} & u_{f z}\end{array}\right]^{T}$ is the vector of control input. In consequence, with the above assumption, Equations (3.9) - (3.11) become

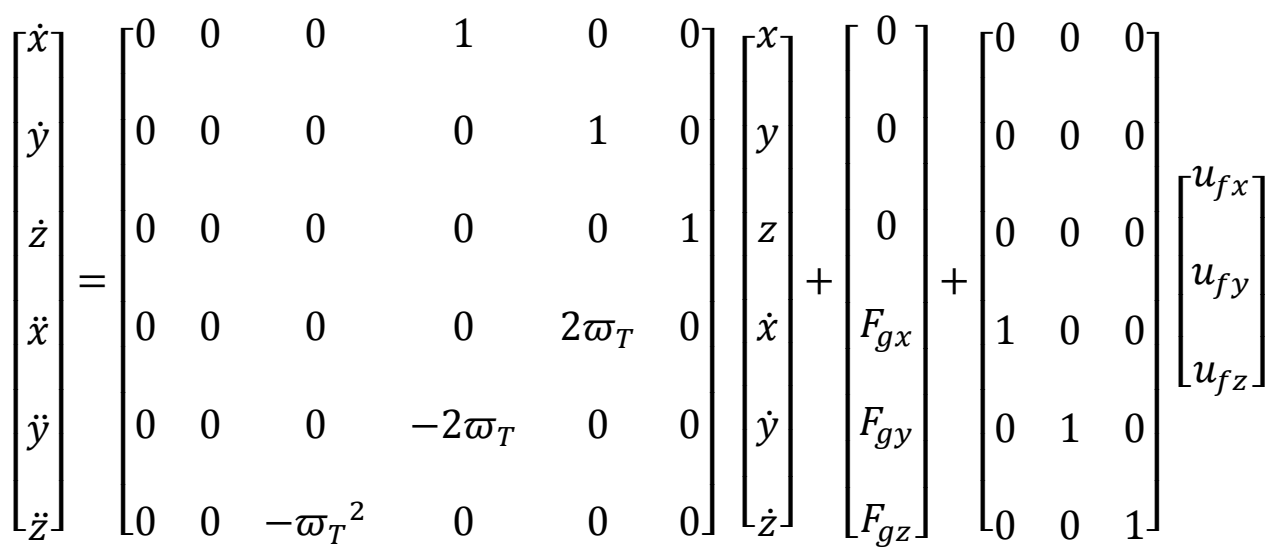


Following the mathematical model presented in the previous section, the relative state vector and the desired relative trajectory are expressed as $X(t)$ and $X_{d}(t) \in \mathbb{R}^{6}$, respectively. The performance measure is then defined as the tracking error $e(t) \in \mathbb{R}^{6}$

$$
\mathrm{e}(t) \triangleq X(t)-X_{d}(t)
$$

The goal of the control law is to reduce tracking errors, with the sufficient input force $\left(u_{f x}, u_{f y}, u_{f z}\right)$ to force the states of the system to its desired reference trajectories as $t \rightarrow \infty$.

$$
\lim _{t \rightarrow \infty} X(t)=X_{d}(t)
$$

\subsubsection{Design of Sliding Manifold}

This section discusses extension of the previous developed single-input single-output (SISO) system in Chapter 3.1.2 to multi-input multi-output (MIMO) systems given in Equation (3.9)-(3.11). We are interested again in the problem of having the state $X$ track a desired timevarying state $X_{d}$. For the control perspective, we utilize the vector containing the trajectory tracking errors, combined with the consensus algorithm, to design a sliding surface $s$ given by

$$
s_{i}=\alpha_{v i}+\mathrm{K}_{1} \alpha_{x i}
$$

The sliding surface $s_{i}$ is a 3 -dimensional manifold, $s_{i} \in \mathbb{R}^{3 \times 1}$ where $K_{1}$ is a positive constant. $\alpha_{x i}$ and $\alpha_{v i}$ are the position lumped error and velocity lumped errors, respectively. Taking the time derivative of Equation (3.17) gives

$$
\dot{s}_{i}=\dot{\alpha}_{v i}+\mathrm{K}_{1} \alpha_{v i}
$$

and Equation (3.17) can be expressed in a compact form as

$$
s=\alpha_{v}+\mathrm{K}_{1} \alpha_{x}
$$


where $\alpha_{v}$ and $\alpha_{x}$ is defined in Equation (2.65) and Equation (2.66), respectively. $s=$ $\left(s_{1}{ }^{T}, s_{2}{ }^{T}, \cdots s_{n}{ }^{T}\right)^{T} \in R^{3 n}$.

\subsubsection{Control Law Formulation}

The control law for the $i$ th follower agent in the multi-agent system is now given by

$$
u_{i}=g_{i}^{-1}\left(-\mathrm{f}_{i}-\mathrm{K}_{2} \operatorname{sgn}\left(s_{i}\right)-\mathrm{K}_{1} s_{i}\right) \quad i=1,2, \cdots, n
$$

where $\mathrm{g}_{i}, \mathrm{f}_{i}$ are defined in Equation (2.56). $K_{1}$ and $K_{2}$ is a positive constant. The compact form of Equation (3.20) can be expressed as follow

$$
u=g^{-1}\left(-F-\mathrm{K}_{2} \operatorname{sgn}(s)-\mathrm{K}_{1} s\right)
$$

where $g$ and $F$ are defined in Equation (2.58)

\subsubsection{Stability Analysis}

In the following section, the stability proof shows that the control law defined by Equation (3.21) for the closed-loop system (2.49) guarantees the chosen sliding manifold converges to zero in finite time.

Theorem 3.1: For the multiple spacecraft formation flying mathematical model in Equation (2.48) to (2.49), the sliding manifold is chosen as Equation (3.19), the control law is defined as Equation (3.21), and the bounds on the external disturbances on the system is assumed as given by Equation (2.34) to (2.36). For a sufficiently large positive constant $V_{m}$, if the initial conditions satisfy

$$
s^{T}(0) s(0) \leq \frac{2 V_{m}}{\lambda_{\max }\left(M^{-1}\right)}
$$

and $\alpha_{x}(0)$ is bounded, then the closed-loop system is semi-global stable; s converges to zero in finite time, and $\alpha_{x}$ and $\alpha_{v}$ converge to zero as $t$ goes to infinite. 
Proof: Consider the following Lyapunov function,

$$
V=\frac{1}{2} s^{T} M^{-1} S
$$

taking the time derivative yields

$$
\dot{V}=s^{T} M^{-1} \dot{S}
$$

With the time derivative of the sliding manifold from Equation (3.19) as shown below

$$
\dot{s}=\dot{\alpha}_{v}+\mathrm{K}_{1} \dot{\alpha}_{x}=\dot{\alpha}_{v}+\mathrm{K}_{1} \alpha_{v}
$$

multiply both side of Equation (3.25) by $M^{-1}$ to obtain

$$
M^{-1} \dot{s}=M^{-1} \dot{\alpha}_{v}+\mathrm{K}_{1} M^{-1} \alpha_{v}
$$

By substituting the first term on the right-hand side of Equation (3.26) with the corresponding parameters from Equation (2.68), the term $M^{-1} \dot{s}$ can be expressed as follows

$$
M^{-1} \dot{s}=\mathrm{F}+g u+\vartheta-X_{d}+\mathrm{K}_{1} M^{-1} \alpha_{v}
$$

Taking the time derivative of the Lyapunov function, Equation (3.23), and substituting Equation (3.27) and the control law (3.21) yields

$$
\dot{V}=-\mathrm{K}_{2} s^{T} \operatorname{sgn}(s)-\mathrm{K}_{1} s^{T} s+s^{T}\left(\vartheta-X_{d}\right)+\mathrm{K}_{1} s^{T} M^{-1} \alpha_{v}
$$

By applying the following inequality

$$
-\mathrm{K}_{2} s^{T} \operatorname{sgn}(s) \leq-\mathrm{K}_{2}\|s\|
$$


to Equation (3.28), the time derivative of Lyapunov function can be rewritten as follows

$$
\dot{V} \leq-\mathrm{K}_{2}\|s\|-\mathrm{K}_{1}\|s\|^{2}+s^{T}\left(\vartheta-X_{d}+\mathrm{K}_{1} M^{-1} \alpha_{v}\right)
$$

As mentioned in Equations (2.50) and (2.51), $\|\vartheta\|$ and $\left\|X_{d}\right\|$ are bounded. Moreover, $\left\|\alpha_{v}\right\|$ is bounded, based on Lemma 3.1. Accordingly, the following inequality can be obtained.

$$
\left\|\vartheta-X_{d}+\mathrm{K}_{1} M^{-1} \alpha_{v}\right\| \leq \sigma
$$

By applying the above inequality to Equation (3.30), the Equation (3.30) can be rewritten as follows

$$
\dot{V} \leq-\left(\mathrm{K}_{2}-\sigma\right)\|s\|-\mathrm{K}_{1}\|s\|^{2}
$$

If $\mathrm{K}_{2}$ is chosen such that $\mathrm{K}_{2}>\sigma$, and applying

$$
\|s\|=\sqrt{S^{T} S}=\sqrt{\frac{1}{\lambda_{\min }\left(M^{-1}\right)} \lambda_{\min }\left(M^{-1}\right) S^{T} S}
$$

with Equation (3.23), where $\lambda_{\min }\left(M^{-1}\right) s^{T} s=2 V$, to Equation (3.32), the following inequality can be derived

$$
\dot{V} \leq-\left(\mathrm{K}_{2}-\sigma\right) \sqrt{\frac{2}{\lambda_{\min }\left(M^{-1}\right)}} V^{\frac{1}{2}} \leq 0
$$

which implies that s converges to zero in finite time. Since $s=\alpha_{v}+\mathrm{K}_{1} \alpha_{x}$, it can be concluded that $\alpha_{x}$ and $\alpha_{v}$ converge to zero as $t$ approaches to infinity. 


\section{Remark:}

Note that if $\alpha_{x}$ and $\alpha_{v}$ converge to zero, then $e_{x i}=e_{v i}=\alpha_{x i}=\alpha_{v i}=0(i=1,2, \cdots, n)$ can be obtained, which implies that all agents' states $x_{i}$ and $v_{i}(i=1,2, \cdots, n)$ approach their own reference trajectories.

Lemma 3.1: Suppose that $s$ is bounded such that

$$
\|s\| \leq s_{m}=\sqrt{\frac{2 V_{m}}{\lambda_{\max }\left(M^{-1}\right)}}
$$

and $\alpha_{x}(0)$ is bounded, where $V_{m}$ is a sufficiently large number, and $\lambda_{\max }(*)$ is the maximum eigenvalue of a matrix. Then, $\alpha_{x}(t)$ and $\alpha_{v}(t)$ are bounded for all $t$.

Proof: Consider the following Lyapunov function

$$
V=\frac{1}{2} \alpha_{x}^{T} \alpha_{x}
$$

and rearrange Equation (3.19) in term of $\alpha_{v}$, then substituted to the time derivative of Equation (3.36) yield

$$
\dot{V}=\alpha_{x}^{T} \alpha_{v}=\alpha_{x}^{T} s-\mathrm{K}_{1} \alpha_{x}^{T} \alpha_{x}
$$

With Equation (3.35), Equation (3.37) can be expressed as

$$
\dot{V} \leq\left\|\alpha_{x}\right\| s_{m}-\mathrm{K}_{1}\left\|\alpha_{x}\right\|^{2}=-\left\|\alpha_{x}\right\|\left(\mathrm{K}_{1}\left\|\alpha_{x}\right\|-s_{m}\right) \leq 0
$$

In Equation (3.38), $\dot{V} \leq 0$, if

$$
\left\|\alpha_{x}\right\| \geq \frac{s_{m}}{\mathrm{~K}_{1}}
$$


which implies that $\alpha_{x}$ converges to the region $\left\|\alpha_{x}\right\| \leq s_{m} / \mathrm{K}_{1}$ in finite time. Thus, $\alpha_{x}$ can be bounded by

$$
\left\|\alpha_{x}\right\| \leq \max \left\{\alpha_{0} \quad, \quad \frac{s_{m}}{\mathrm{~K}_{1}}\right\}=\alpha_{x \max }
$$

where $\alpha_{0}=\left\|\alpha_{x}(0)\right\|$. This also implies that $\alpha_{v}$ will be bounded.

\subsection{RESUlTS AND DisCUSSIONS}

To study the effectiveness and performance of the proposed consensus control strategies, a comprehensive response is numerically simulated. The simulation is performed by applying the set of governing equations of motion, Equation (3.9) - (3.11), with the perturbation of slowly rotating and irregularly shaped body, Equation (3.12). The formation reference trajectory is in an elliptical orbit with perigee altitude of $3 \mathrm{~km}$. Results are obtained by applying the proposed control law (3.21) with the following parameters, $m_{s}=1 \mathrm{~kg}, \Omega=i=0^{\circ}, e=0, \omega=4.2883 \times$ $10^{-4} \mathrm{rad} / \mathrm{s}, \mu=9.4 \times 10^{-8} \mathrm{~km}^{3} / \mathrm{s}^{2}, C_{20}=-7.275 \times 10^{-2}$, and $C_{22}=2.984 \times 10^{-2}$. There are 6 spacecraft in the consensus network, and the external disturbance acting on each agent is different. These disturbance forces are considered to be time variant [64], with units of Newton, and given by

$$
\left[\begin{array}{l}
F_{d x} \\
F_{d y} \\
F_{d z}
\end{array}\right]_{i}=K_{d s}\left[\begin{array}{l}
\sin (i t) \\
\cos (i t) \\
\sin (i t)
\end{array}\right]
$$

where $K_{d s}$ is the weight of the disturbances, wherein $3 \times 10^{-3}$ is used to obtain the following results.

For the proposed consensus algorithm the communication link is defined using graph theory and is characterised by the following network graph and adjacency matrix. The desired shape considered for formation-keeping is a circle with a radius of $0.5 \mathrm{~km}$. Since the 6 agents are evenly distributing within a circle, 6 equilateral triangles are formed. The shape of the formation becomes a regular hexagon with the side length of $0.5 \mathrm{~km}$, as shown in Figure 3.2. The same formation shape is applied in this thesis if not stated otherwise. 


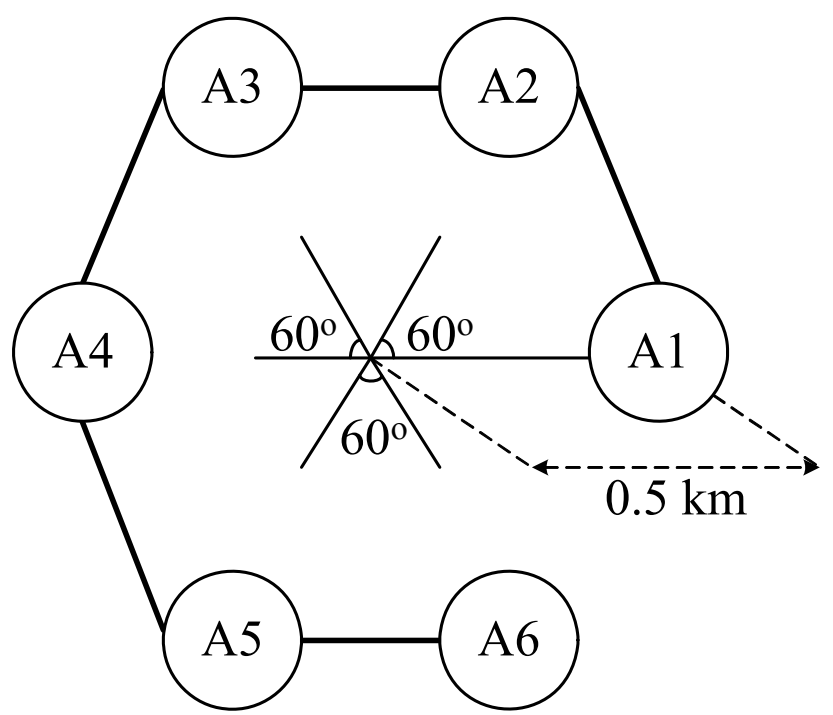

Figure 3.2: Formation Graph $(x-y$ plane; $z=0)$

$$
A=\left[\begin{array}{cccccc}
0 & 0.7 & 0 & 0 & 0 & 0 \\
0.7 & 0 & 0.6 & 0 & 0 & 0 \\
0 & 0.6 & 0 & 0.7 & 0 & 0 \\
0 & 0 & 0.7 & 0 & 0.8 & 0 \\
0 & 0 & 0 & 0.8 & 0 & 0.9 \\
0 & 0 & 0 & 0 & 0.9 & 0
\end{array}\right]
$$

Relative position error, $r_{x i j}$, is the difference between the desired relative distance and the relative distance among two agents. For instance, in the above formation reconfiguration, the relative position error between agent 1 and agent 2 is the difference between 0.5 and the actual distance among the two. The following table is the desired relative distance among each agent corresponding to the formation described in Figure 3.2.

Table 3.1: The desired relative distance

\begin{tabular}{|c|c|c||c|c|c|}
\hline Agent & $\mathrm{x}(\mathrm{km})$ & $\mathrm{y}(\mathrm{km})$ & Agent & $\mathrm{x}(\mathrm{km})$ & $\mathrm{y}(\mathrm{km})$ \\
\hline 1 to 2 & 0.25 & 0.433 & 3 to 4 & 0.25 & 0.433 \\
\hline 1 to 3 & 0.75 & 0.433 & 3 to 5 & 0 & 0.866 \\
\hline 1 to 4 & 1 & 0 & 3 to 6 & 0.5 & 0.866 \\
\hline 1 to 5 & 0.75 & 0.433 & & & \\
\hline 1 to 6 & 0.25 & 0.433 & & & \\
\hline 2 to 3 & 0.5 & 0 & 4 to 5 & 0.25 & 0.433 \\
\hline 2 to 4 & 0.75 & 0.433 & & 0.75 & 0.433 \\
\hline 2 to 5 & 0.5 & 0.866 & & & \\
\hline 2 to 6 & 0 & 0.866 & 5 to 6 & 0.5 & 0 \\
\hline
\end{tabular}


The control gains used in all the simulations for the sliding manifold $K_{1}$ and control law $K$ are obtain by trial and error, and the values are 0.05 and $1 \times 10^{-5}$, respectively. Since the control law developed in the subsequent chapter is based on the one constructed above, the values of the orbital elements, spacecraft properties, formation geometry, and consensus properties, as well as other system parameters as defined above, are shared in all chapters. Moreover, a specific scenario, in Chapter 5, is examined using the control law developed in Chapters 3 and 4. Therefore, in order to make a comparison, the same control gains are applied to both chapters.

\subsubsection{Initial State and Mission Scenarios}

The following two sets of initial state values were used in numerical simulation:

Set 1: $x_{1}(0)=(5.25,6,0) \mathrm{km}, v_{1}(0)=(-0.9,0.4,0.2) \mathrm{km} / \mathrm{s} ; x_{2}(0)=(5.35,6,0) \mathrm{km}$, $v_{2}(0)=(0.9,-0.5,0.2) \mathrm{km} / \mathrm{s} ; x_{3}(0)=(5.45,6,0) \mathrm{km}, v_{3}(0)=(-0.9,-0.6,0.2) \mathrm{km} / \mathrm{s} ; x_{4}(0)=$ $(5.55,6,0) \mathrm{km}, v_{4}(0)=(0.9,-0.6,0.2) \mathrm{km} / \mathrm{s} ; x_{5}(0)=(5.65,6,0) \mathrm{km}, v_{5}(0)=(0.9,-0.4,0.2)$ $\mathrm{km} / \mathrm{s} ; x_{6}(0)=(5.75,6,0) \mathrm{km}, v_{6}(0)=(-0.9,0.5,0.2) \mathrm{km} / \mathrm{s}$. This initial states contain large position errors and velocity errors compared to the system desired state. Various cases were investigated and the effects of the position errors are not as significant as those of the velocity errors, therefore, the velocity errors are the major focus when deciding this state error. The position was set to be 0.1 units apart from each other so as to examine whether or not the agents would collide with one another. There are three characteristics in the velocity state error; the velocity error in the $\mathrm{x}$-direction stays the same but alters between positive and negative; the velocity error in the y-direction alters between -0.6 to $0.7 \mathrm{~km} / \mathrm{s}$; and the velocity error in the z-direction remains constant for all agents. It is expected that with these initial state errors, the system responds more chaotically, and more control effort is required to bring the spacecraft back to the desired state than the next set of initial errors. It is also expected that the settling time will be longer if the control gains stay the same.

Set 2: $x_{1}(0)=(0,3.2,0) \mathrm{km}, v_{1}(0)=\left(0,-8.5 \times 10^{-4}, 0\right) \mathrm{km} / \mathrm{s}$; the position of the other agents is distributed $0.1 \mathrm{~km}$ apart from each other similar to set 1 . However, the velocity for each agent remains the same. This is the nominal initial state error, and is used throughout this thesis when the main purpose is not examining how the proposed control law responds to system initial errors. The initial state errors in set 2 is less challenging when compared with set 1 . The second purpose for having the initial position of the six spacecraft lined up closely to each other, i.e. start 
flying as a straight line, is to model a situation when agents are being deployed from the agents' carrier or space shuttle. Initially, the system external disturbance is not applied in order to obtain a baseline response from the developed control methodology.

As mentioned in Chapter 2, in order to increase the realism of the simulation, some mission requirements are added, for example, actuator saturation, sensor faults, and varying system parameter, i.e. the mass of the agents. The maximum allowable thrust is $5 \mathrm{mN}$; the mass of the spacecraft is $1 \mathrm{Kg}$; the settling time, in other words, the state errors have to converge to zero, or less than $10^{-3} \mathrm{~km}$ within one orbit. For system requirement, Sun sensor and Earth sensor will be used to obtain the state of the spacecraft.

In the following section, there are different scenarios where the control law developed above is investigated. The system is first examined with the large initial errors, and the results for the nominal initial errors is shown in Chapter 3.2.4 as the preliminary for later cases. In Chapter 3.2.3, the disturbances model explained in Chapter 2.1.5 is applied to those cases in Chapter 3.2.2. The nominal initial state error is used throughout Chapter 3.2.4 to discover the effect of the pure sensor faults. Various sensor defects is modeled, and disturbances is not included. Last, Chapter 3.2.5 examines the robustness of the control law when the mass of the spacecraft changes during the mission flight.

\subsubsection{Effect of Large Initial State Errors}

In this chapters, numerical simulations are carried out to demonstrate the effectiveness of the developed control law. The nonlinear control parameters used are $K_{1}=0.05 K=1 \times 10^{-5}$. Simulations are conducted to discover the significant effects of the actuator saturation with the influence of the set 1 state error. Three cases are shown in Figure 3.3 to Figure 3.5, where the maximum allowable forces from the thruster are infinite, $10 \mathrm{mN}$, and $5 \mathrm{mN}$, correspondingly. 


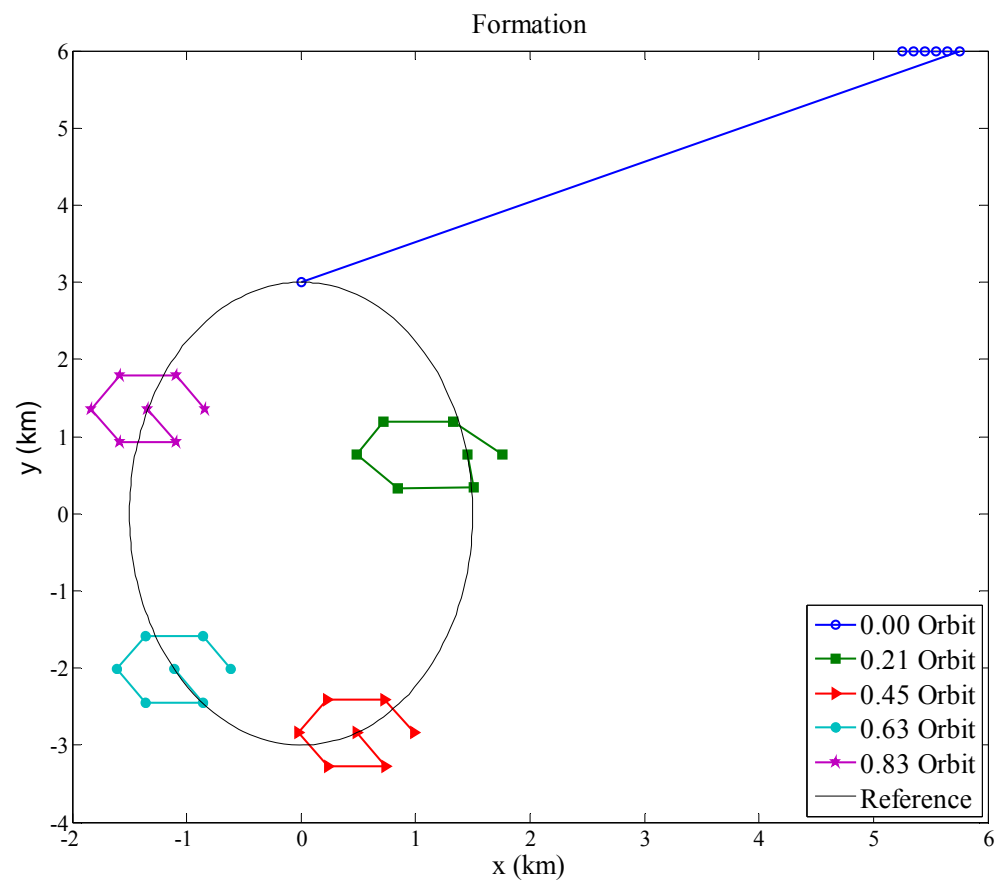

Figure 3.3: System state snap shot at different time (without thruster saturation)

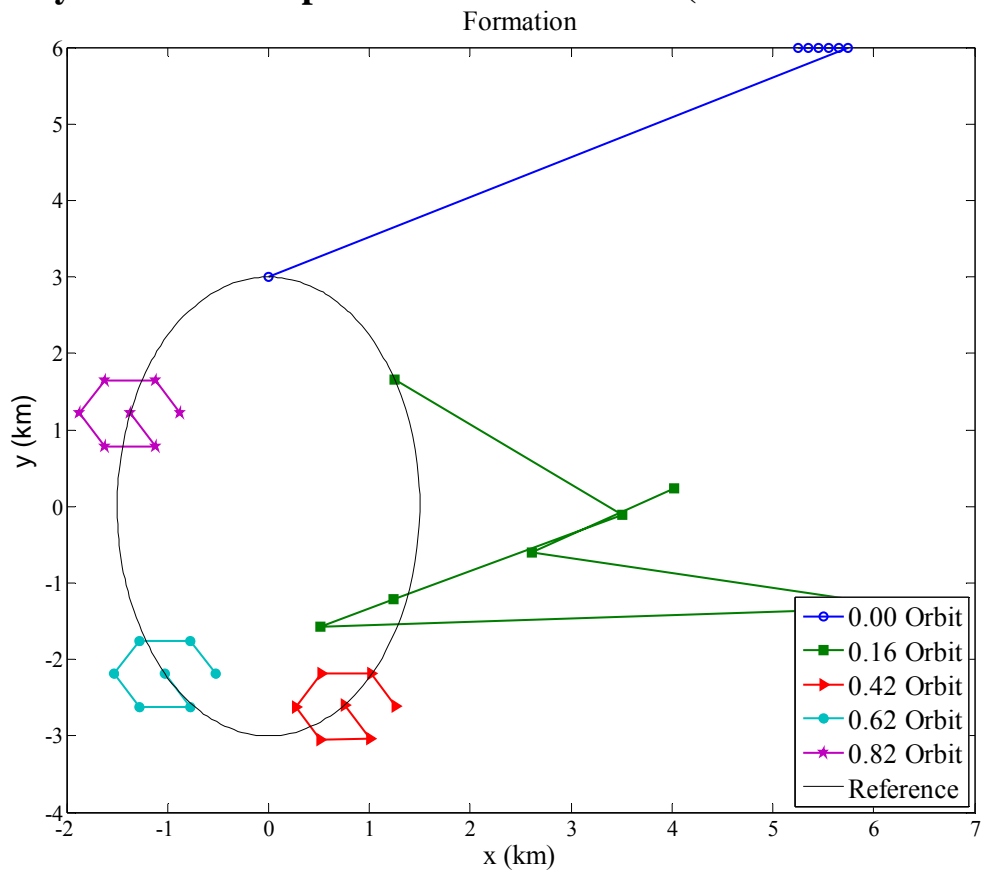

Figure 3.4: System state snap shot at different time (with $10 \mathrm{mN}$ thruster saturation) 


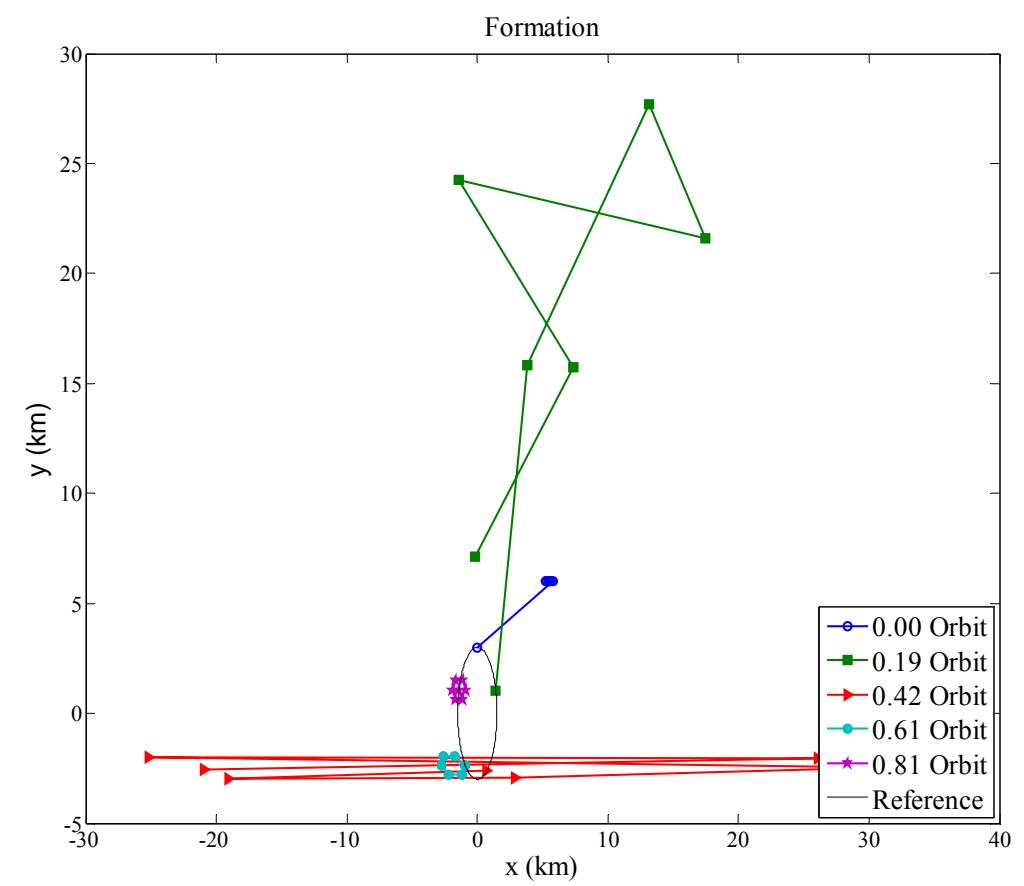

Figure 3.5: System state snap shot at different time (with $5 \mathrm{mN}$ thruster saturation)

For all cases, the proposed control law is able to bring all 6 agents back to the desired state within 1 orbit. When infinite amount of actuation force is available, this control law is able to configure all 6 agents to its desired state at 0.30 of an orbit. The settling time extends from 0.30 to 0.42 of an orbit when there is only $10 \mathrm{mN}$ of thruster forces supplied. Corresponding to Figure 3.3, the relative position error among each agent is shown in Figure 3.6. 


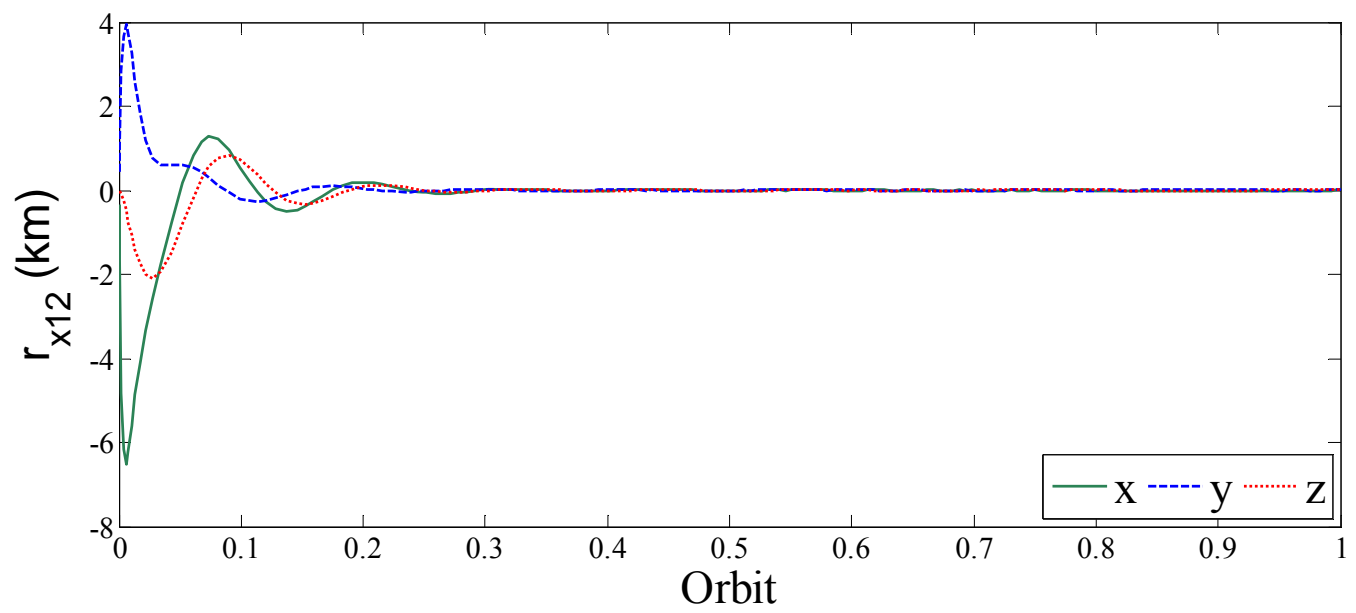

(a)

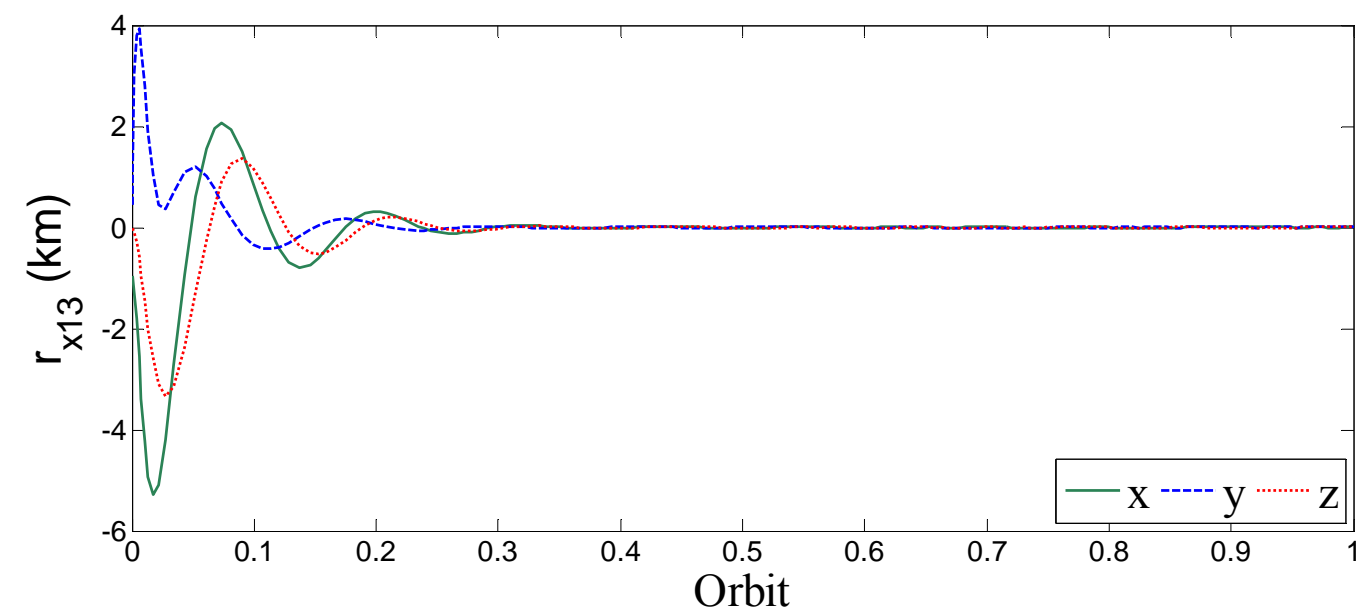

(b)

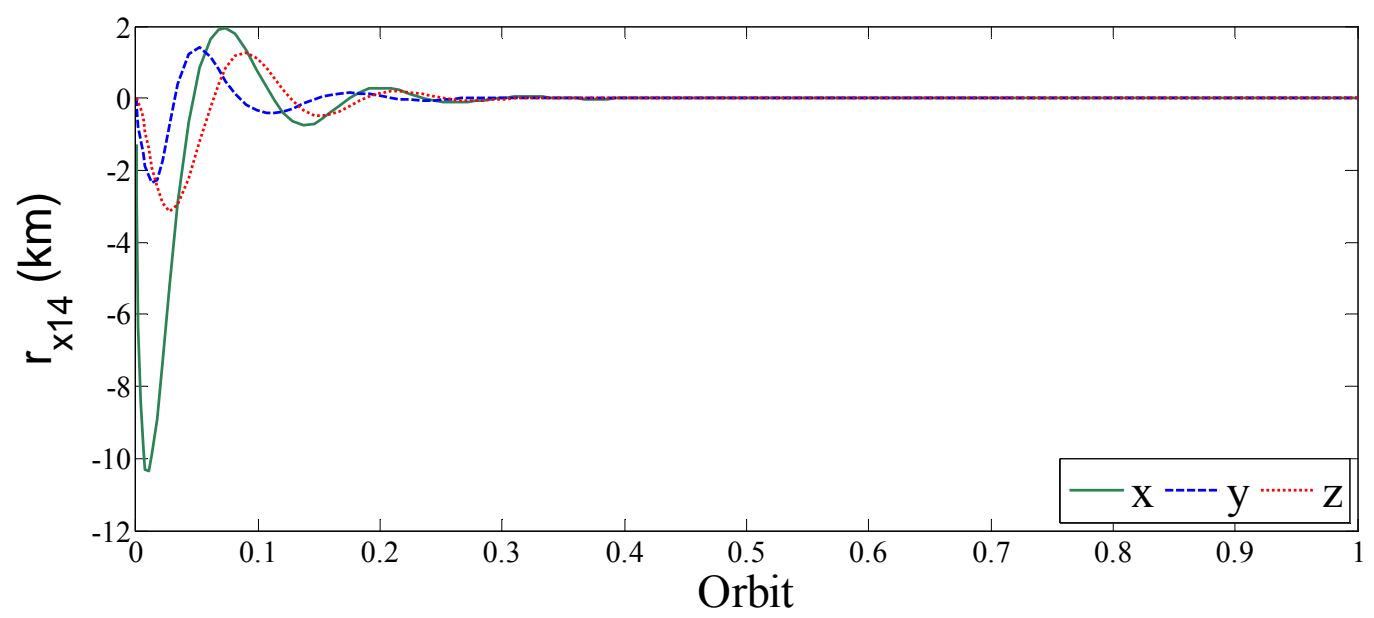

(c)

Figure 3.6: Relative position error (without thruster saturation)

(a) agent 1 to 2; (b) agent 1 to 3; (c) agent 1 to 4 


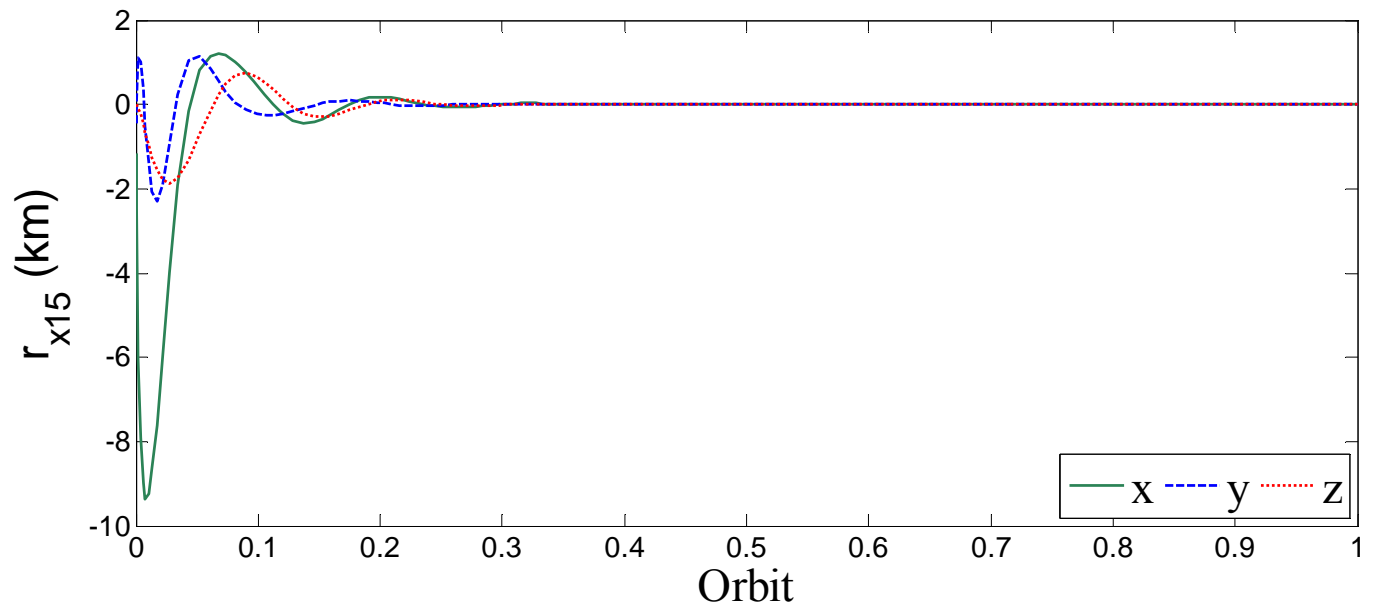

(d)

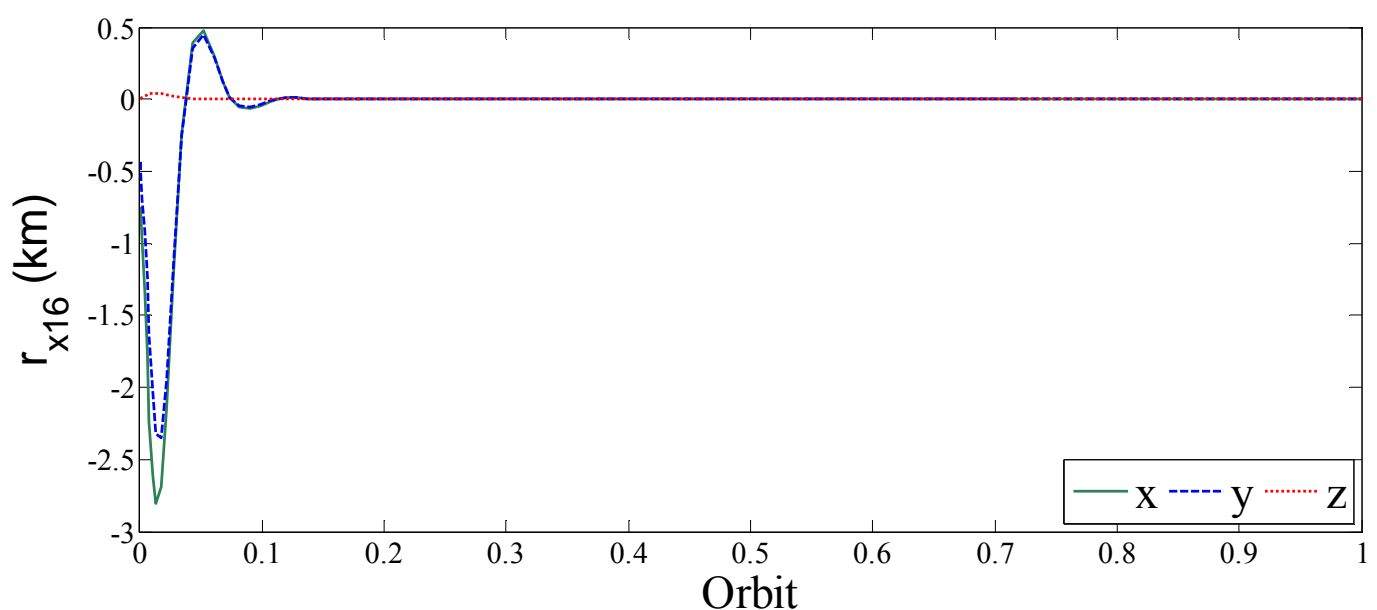

(e)

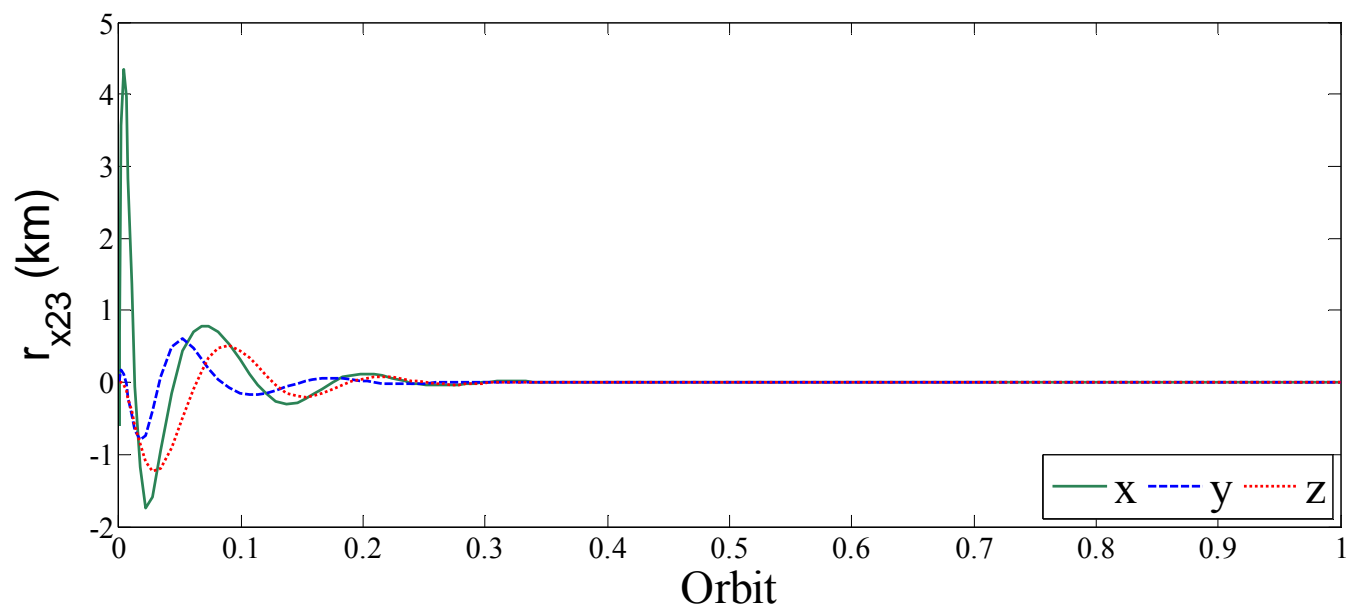

(f)

Figure 3.6 (Cont.): Relative position error (without thruster saturation) (d) agent 1 to 5; (e) agent 1 to 6; (f) agent 2 to 3 


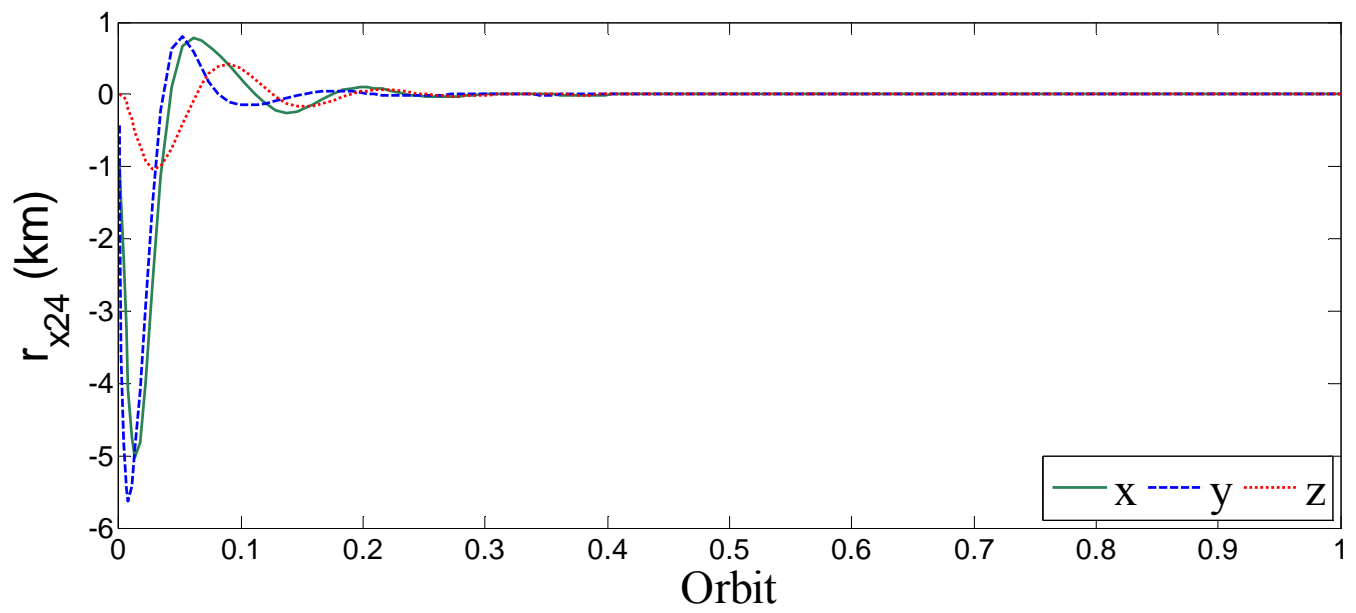

(g)

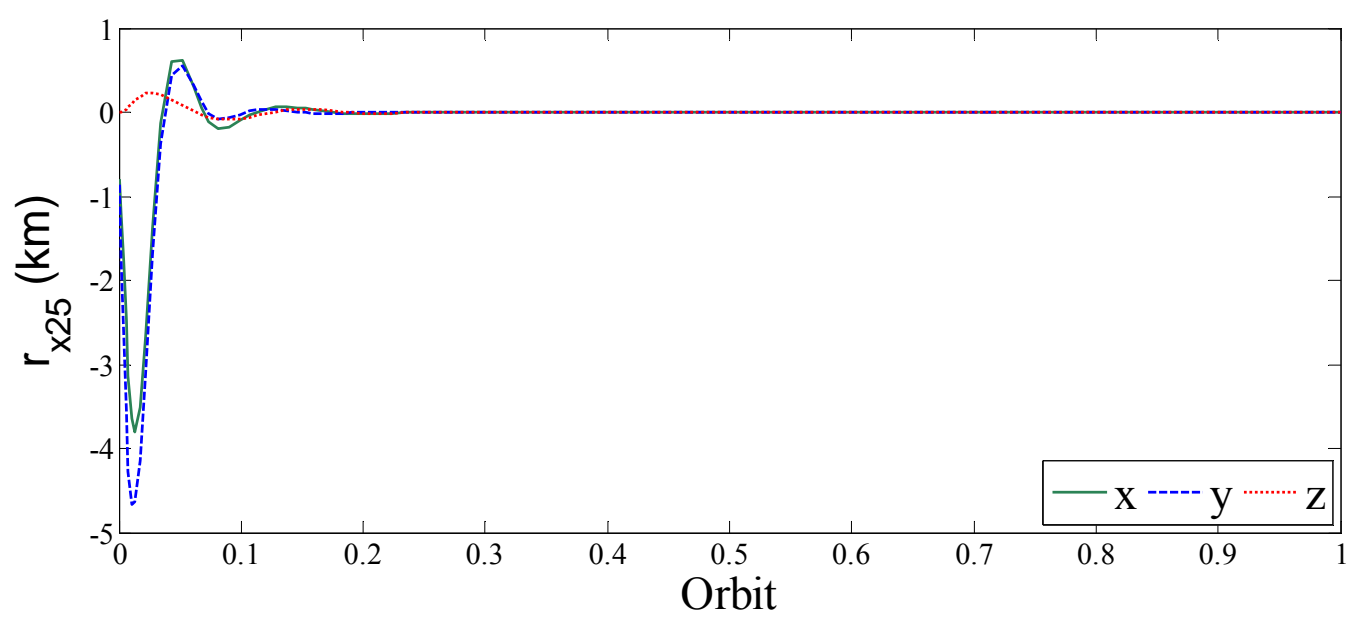

(h)

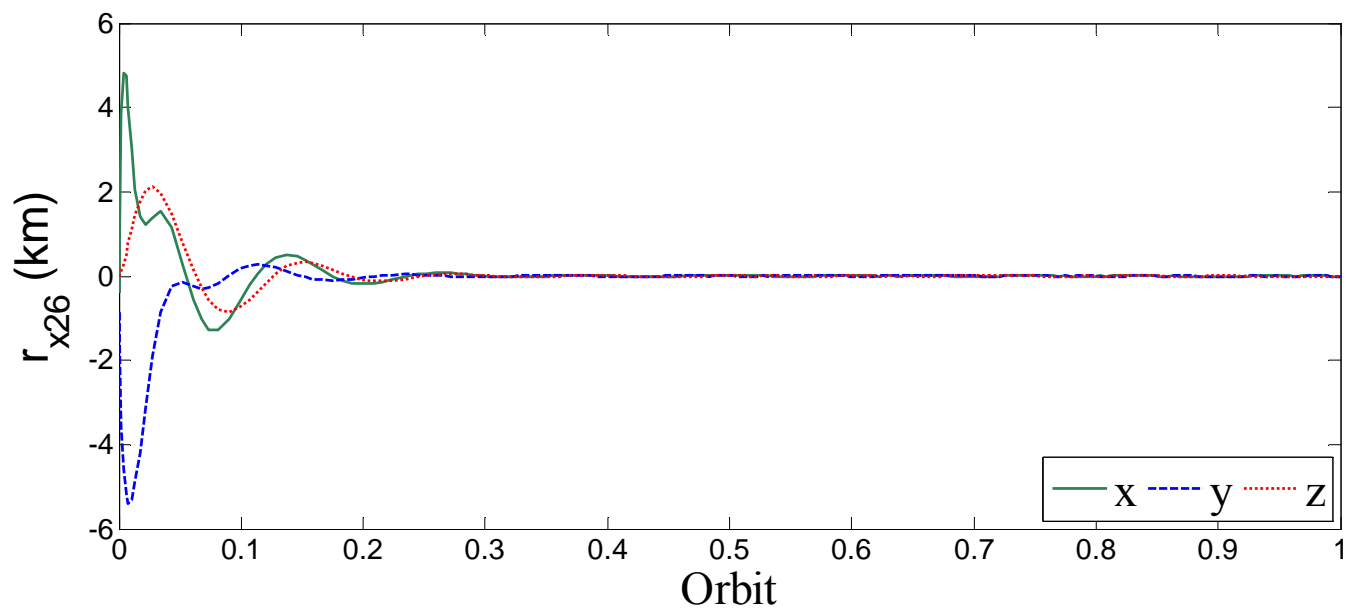

(i)

Figure 3.6 (Cont.): Relative position error (without thruster saturation)

(g) agent 2 to 4; (h) agent 2 to 5; (i) agent 2 to 6 


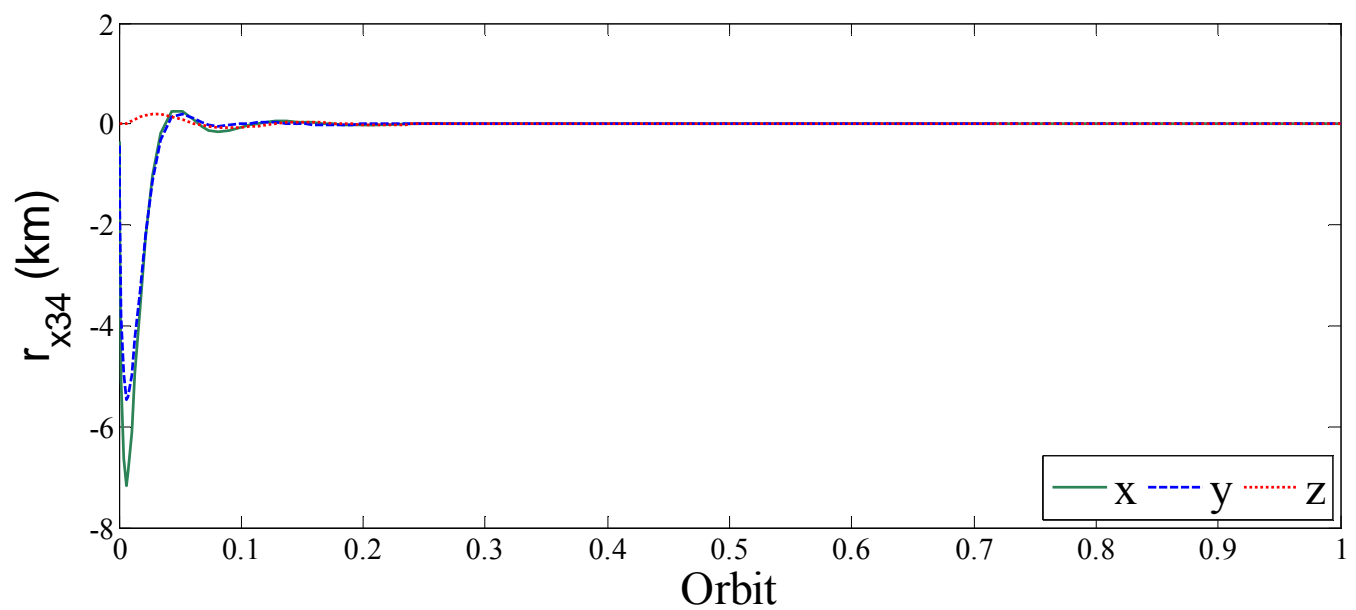

(j)

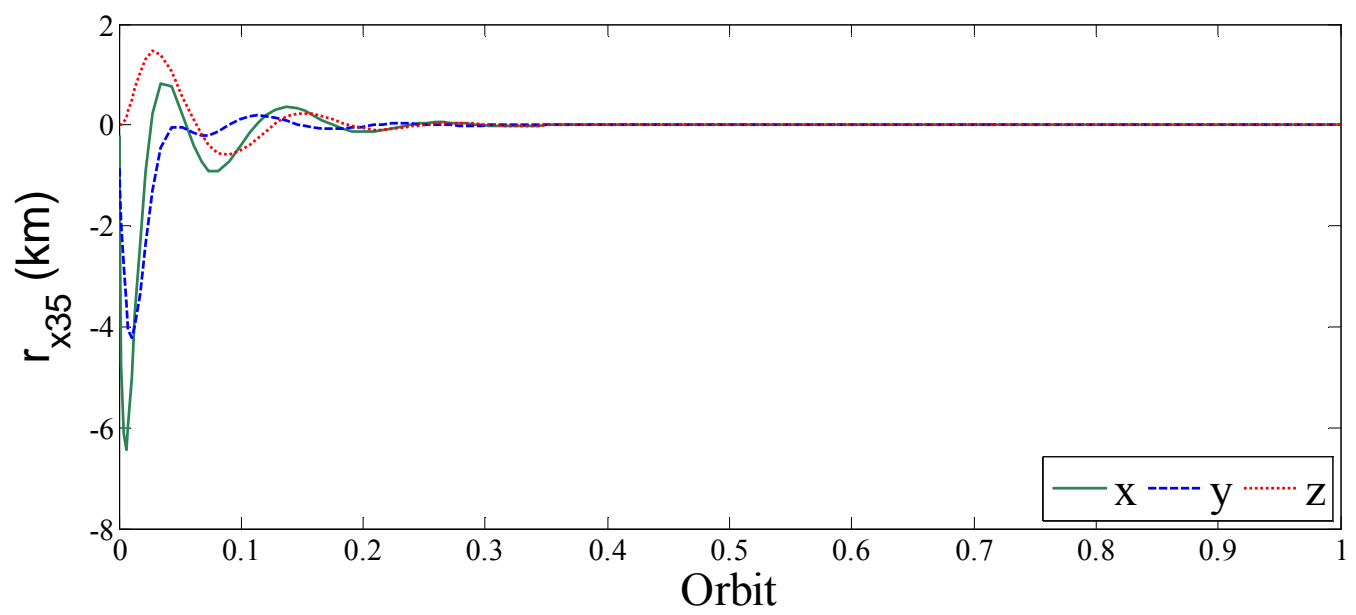

(k)

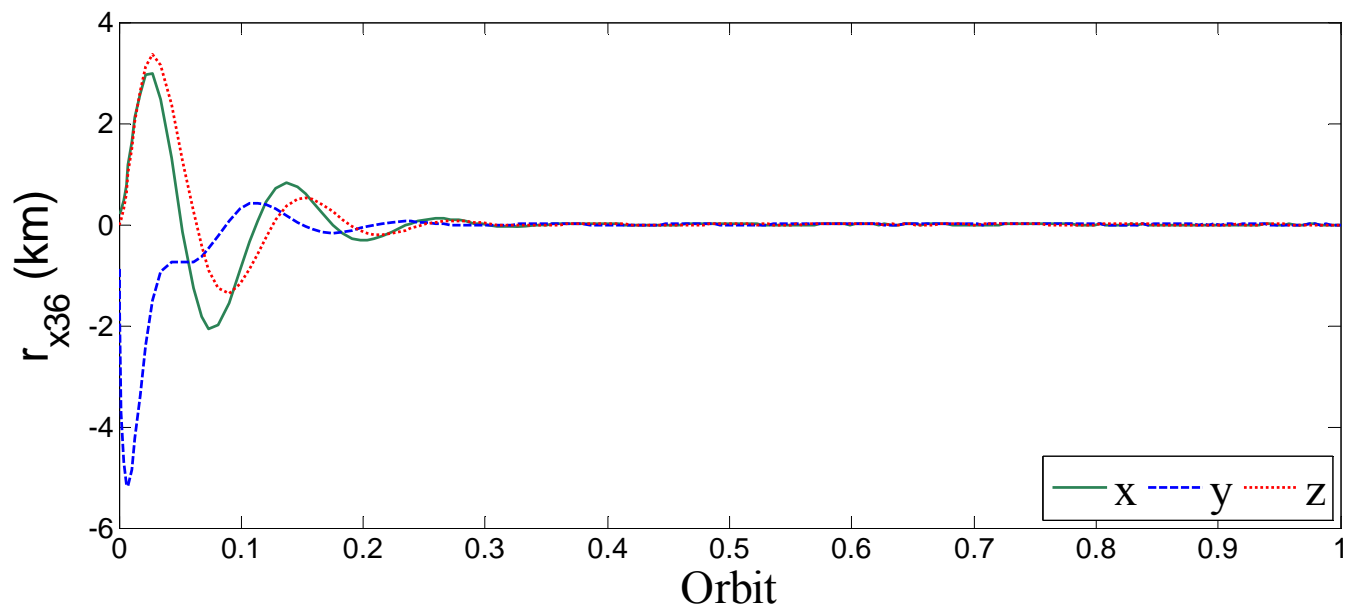

(1)

Figure 3.6 (Cont.): Relative position error (without thruster saturation) (j) agent 3 to 4; (k) agent 3 to 5; (l) agent 3 to 6 


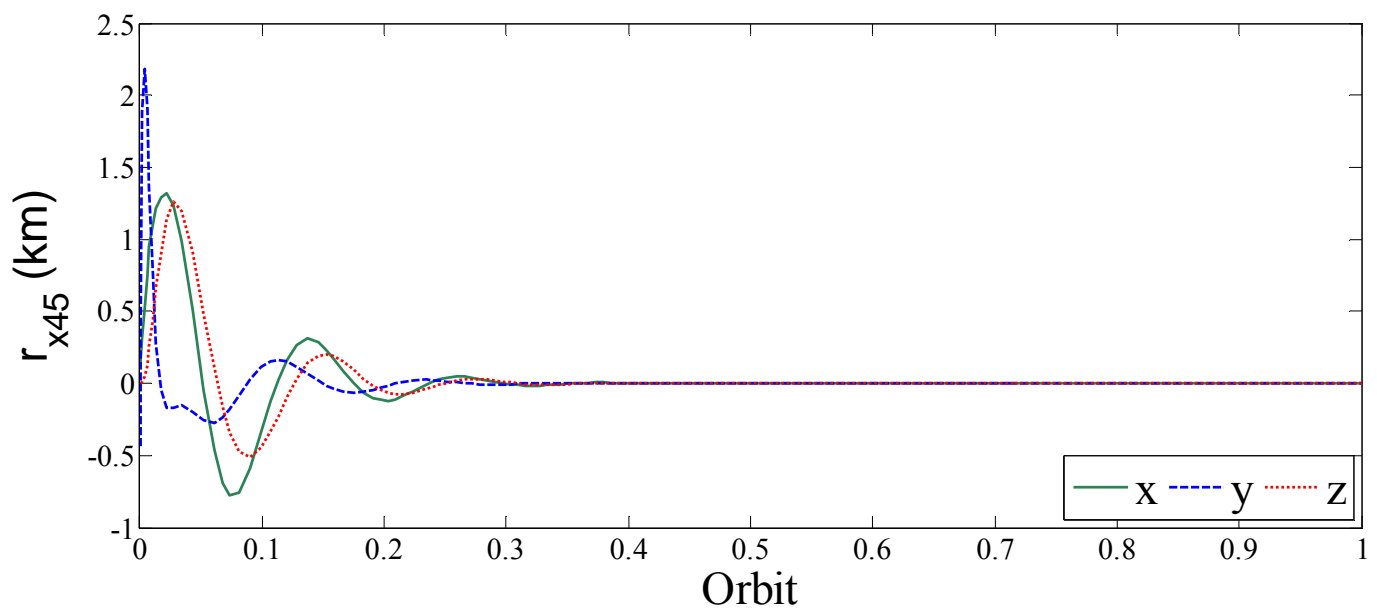

(m)

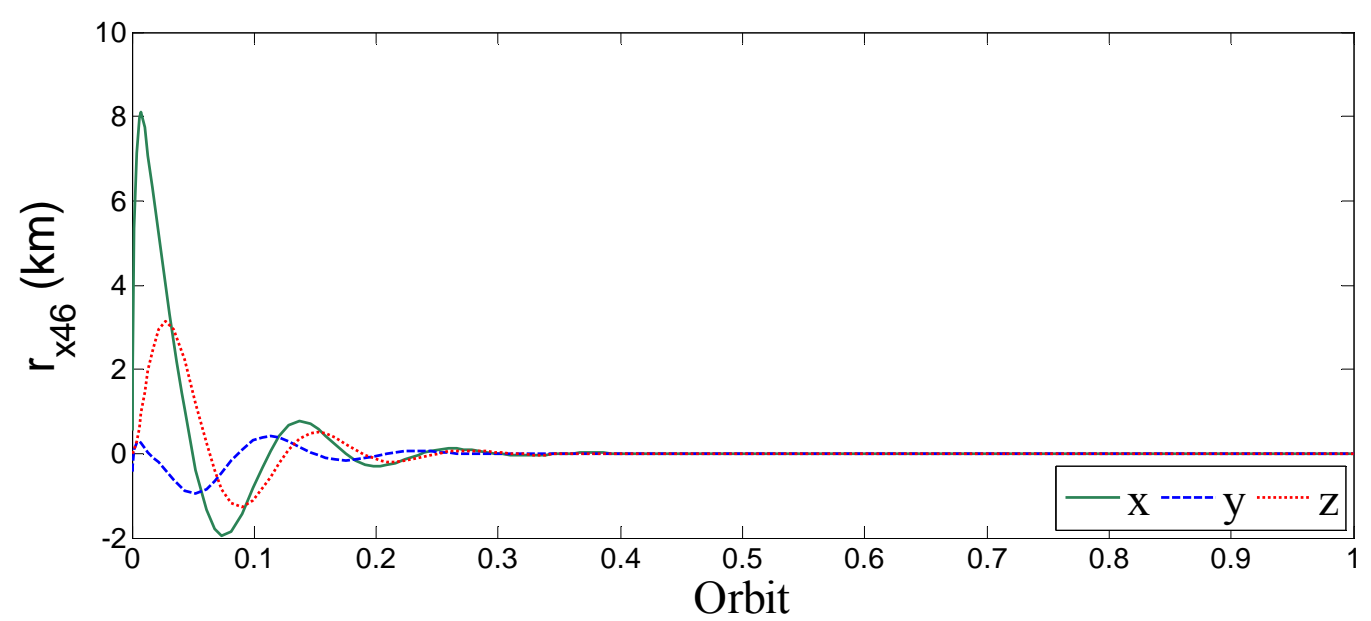

(n)

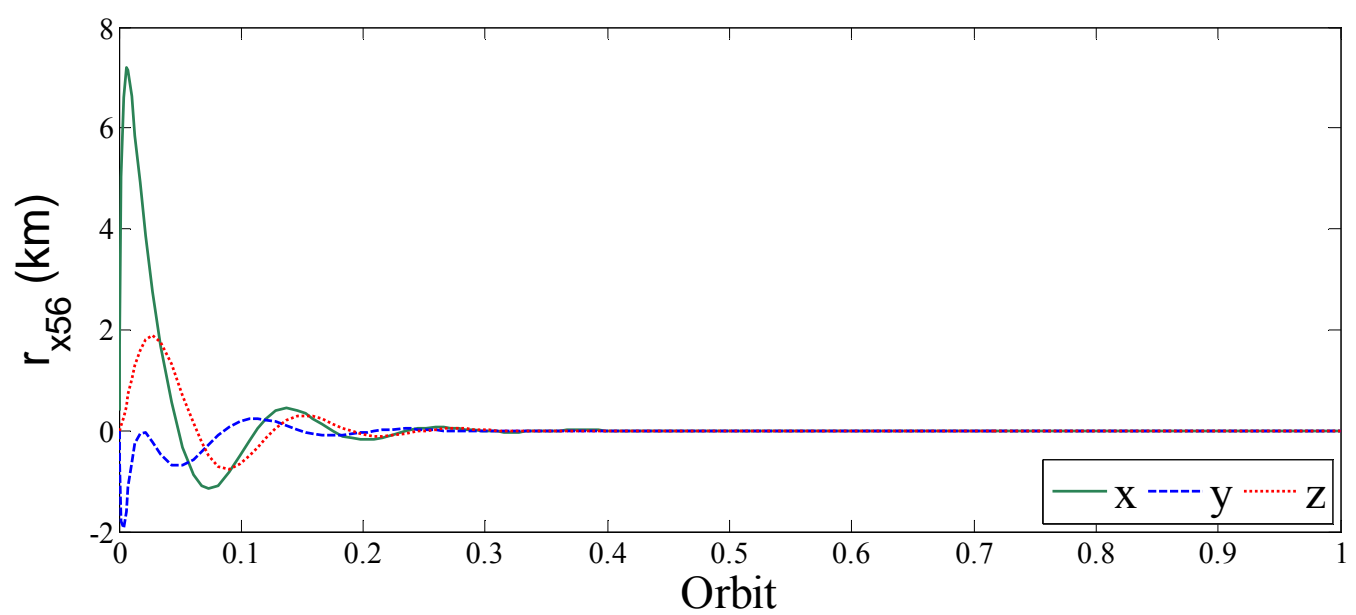

(o)

Figure 3.6 (Cont.): Relative position error (without thruster saturation) (m) agent 4 to 5; (n) agent 4 to 6; (o) agent 5 to 6 


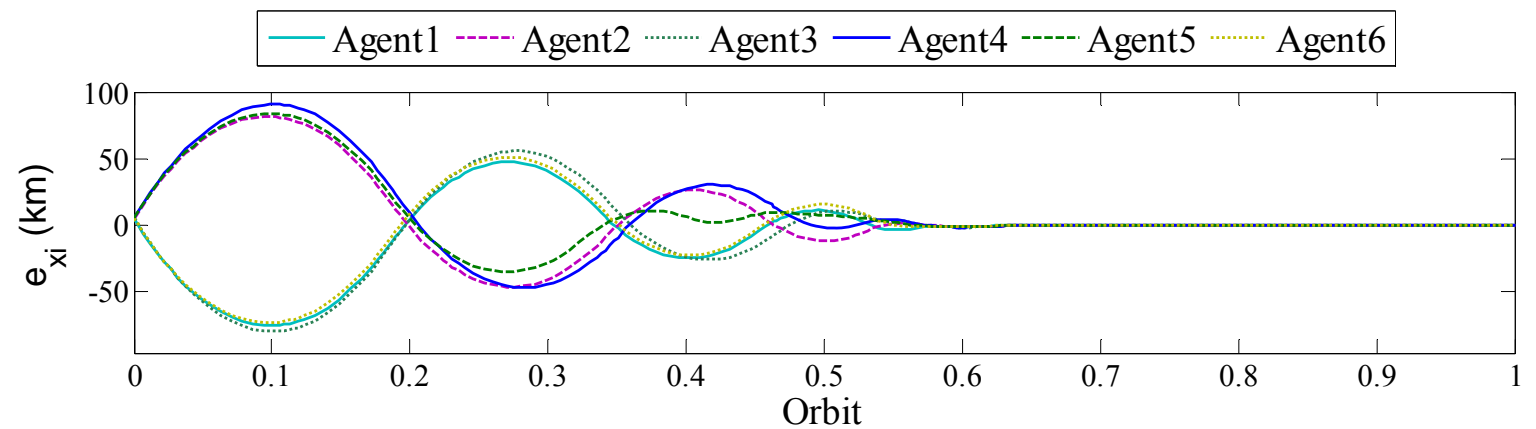

(a)

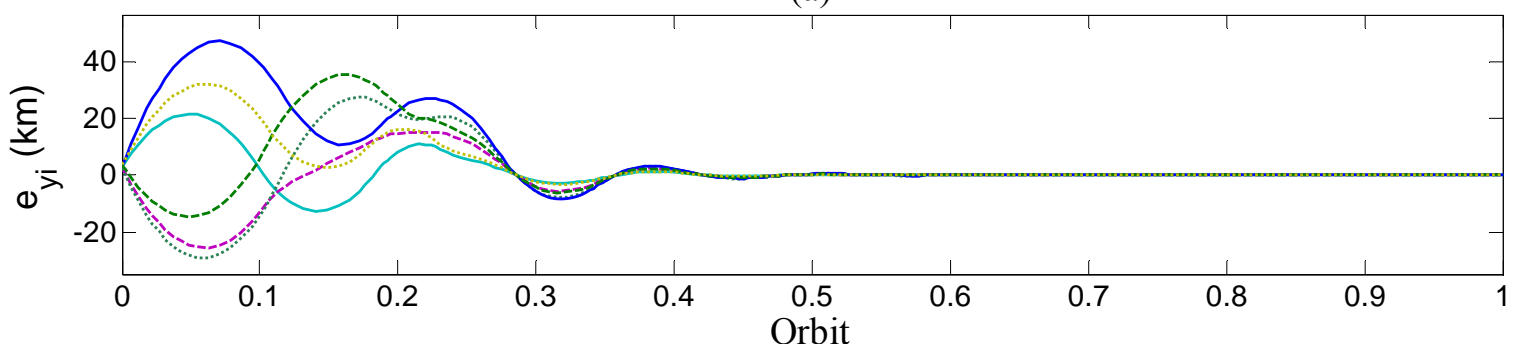

(b)

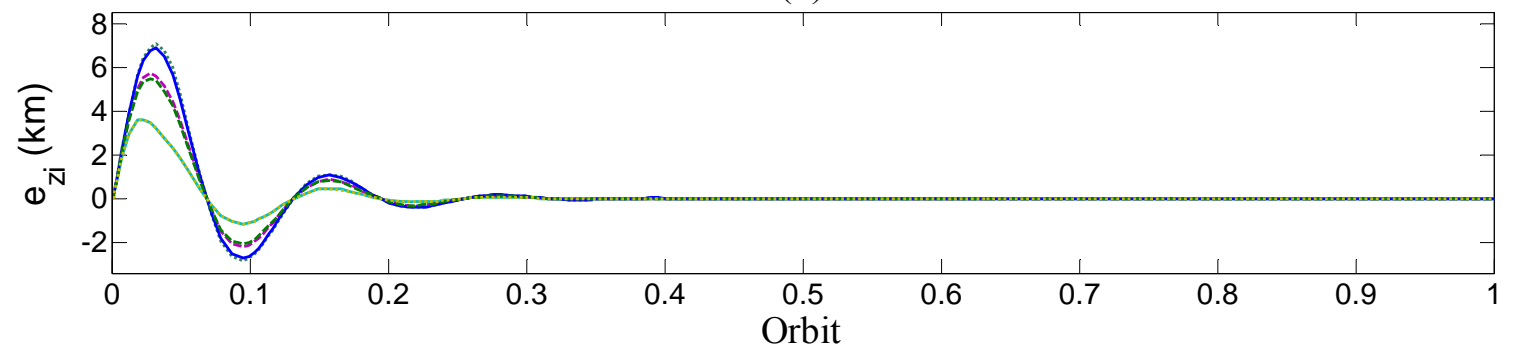

(c)

Figure 3.7: Tracking Errors (with $5 \mathrm{mN}$ thruster saturation)

As expected when there is less force supplied from the thruster, the settling time is longer. When there is only $5 \mathrm{mN}$ of actuator force, the settling time is extended to 0.7 of an orbit, as shown by the tracking error in Figure 3.7.

Though, for the first case, we said there is an infinite amount of thruster force provided, the maximum thrust that the system required is only $0.25 \mathrm{~N}$ which is the initial force required in the x-direction due to the state error, see Figure 3.8 (a). And it quickly converges to less than $5 \mathrm{mN}$ in less than $1 / 10^{\text {th }}$ of an orbit. 


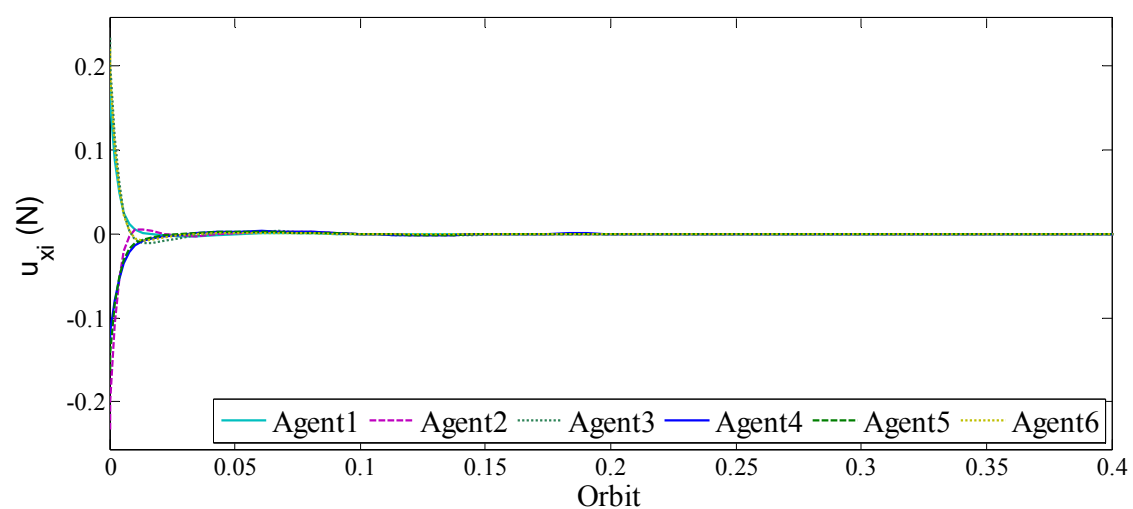

(a)

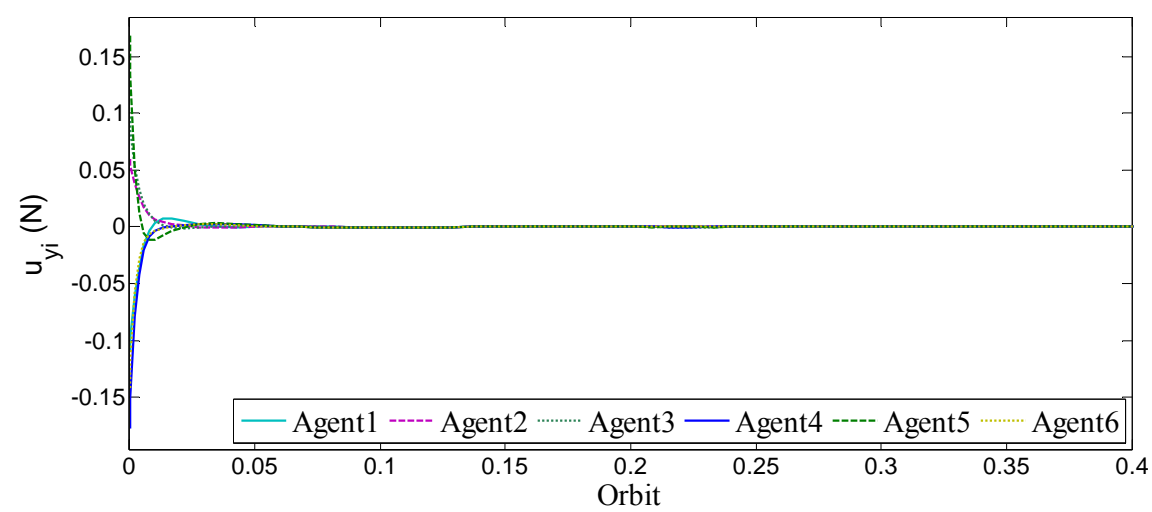

(b)

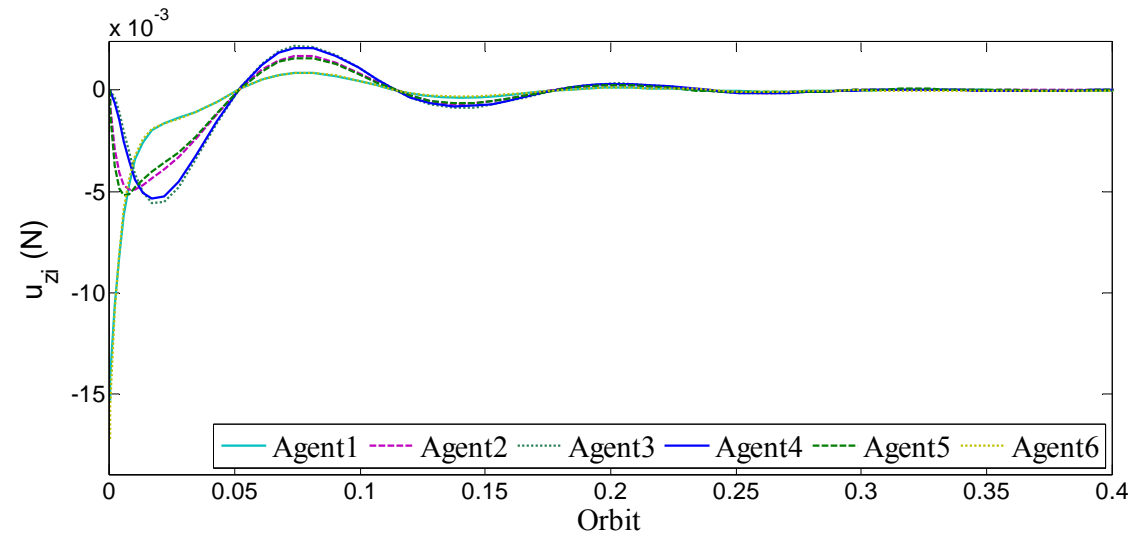

(c)

Figure 3.8: Control input (without thruster saturation)

In Figure 3.9, $10 \mathrm{mN}$ maximum allowable thrust is applied. The response of the system control input matches with the system map in Figure 3.4. Due to the $10 \mathrm{mN}$ actuator saturation, agents need more time to reach their desired states, and clearly, a constant maximum thrust is required until 0.1 and 0.05 of an orbit for $\mathrm{x}$ and $\mathrm{y}$ directions, respectively. For the $5 \mathrm{mN}$ saturation case, the constant maximum force is required until 0.6 and 0.4 of an orbit for $\mathrm{x}$ and $\mathrm{y}$ directions, respectively. 


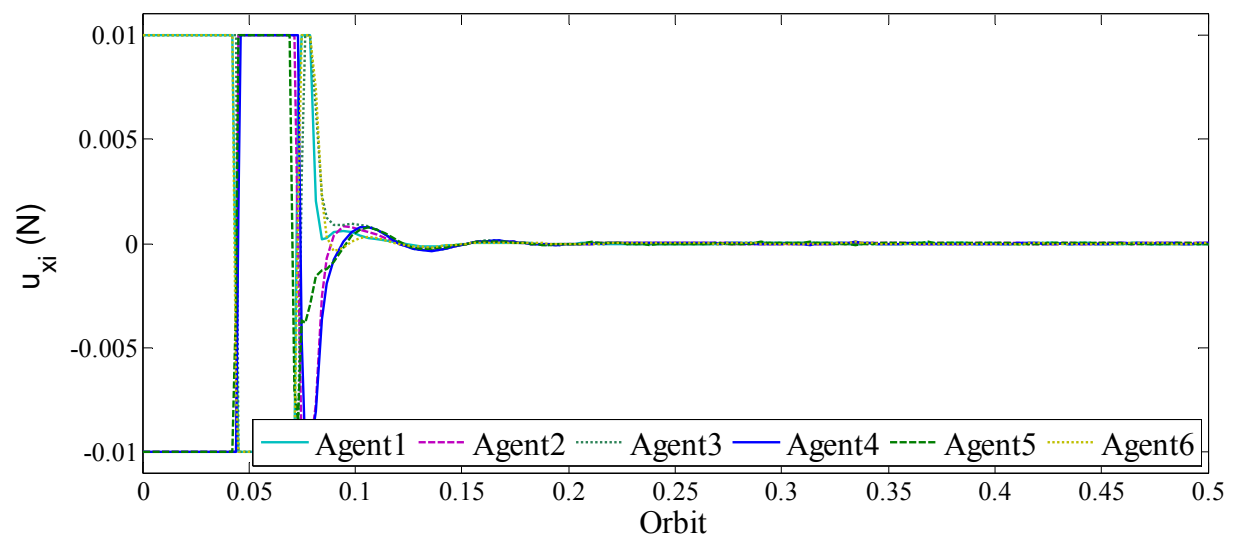

(a)

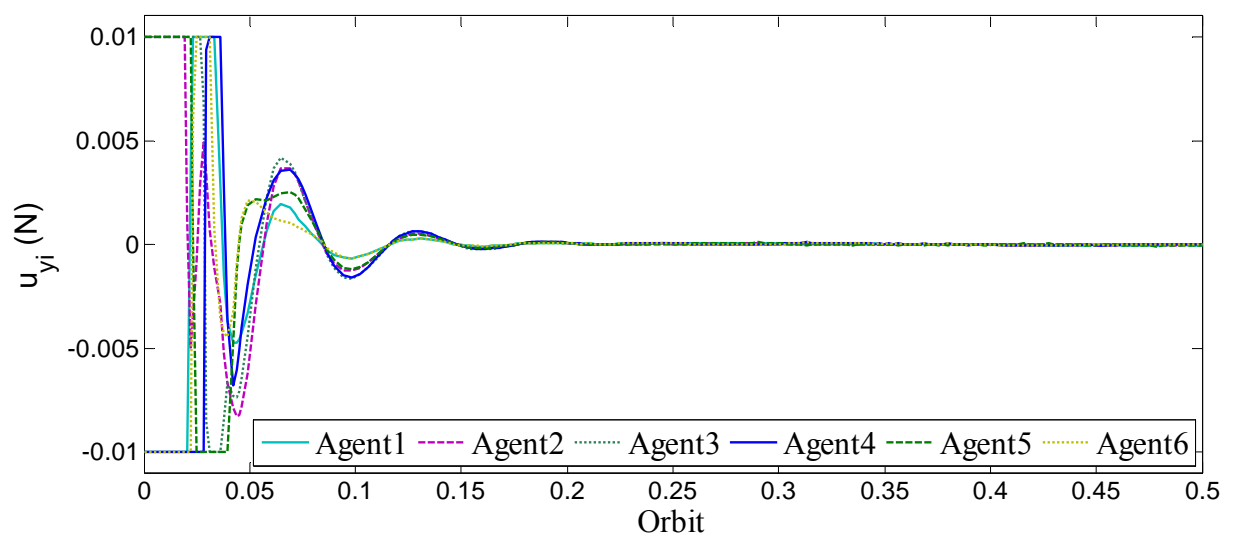

(b)

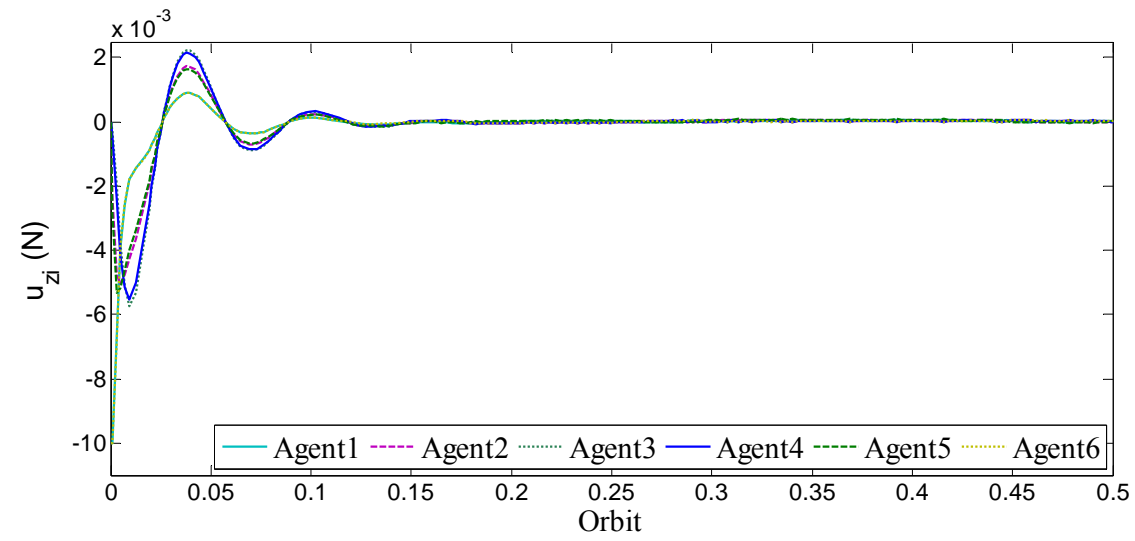

(c)

Figure 3.9: Control input (with $10 \mathrm{mN}$ thruster saturation)

Depending on the value of the thruster saturation, formation is formed and the desired state is reached for all three cases. When modelling practical mission scenarios, the system responds differently in the process of reaching its desired state and yet, the control law is capable of accomplishing the mission objective. 


\subsubsection{Effect of Initial State Errors with Disturbances}

The same situation defined in Chapter 3.2.2 is simulated again here, with the addition of the disturbance model introduced in Chapter 2.1.5. When there is more than $5 \mathrm{mN}$ of maximum allowable thrust provided to the system, all 6 agents are controlled and the system state errors converge to a very small value which is close to zero. This is shown in the result of the tracking errors, Figure 3.10 and Figure 3.11.
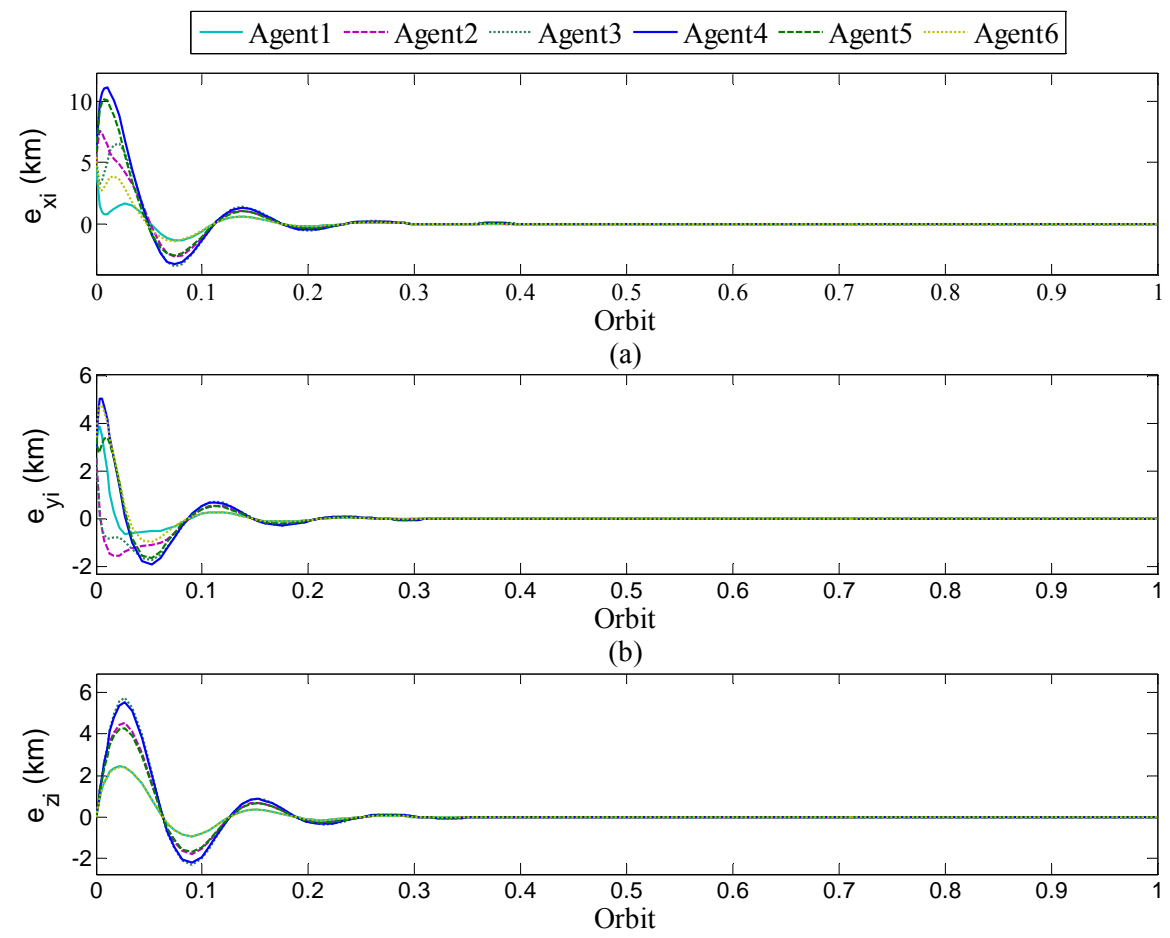

(c)

Figure 3.10: Tracking errors (without thruster saturation)

The required control effort for both scenarios are disclosed in Figure 3.12 and Figure 3.13. Each agent requires different amounts of control input when experiencing different frequencies of disturbance. As explained in Figure 2.8, because of the large disturbance force compare with the system dynamics, the control efforts shown are counterbalancing the disturbances. 

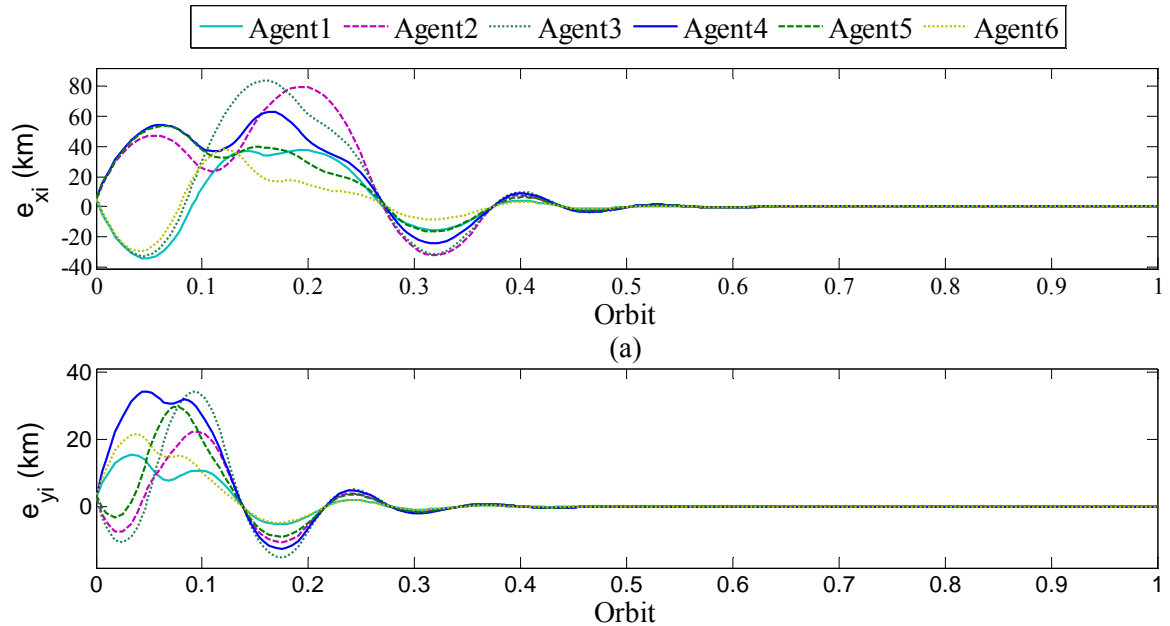

(b)

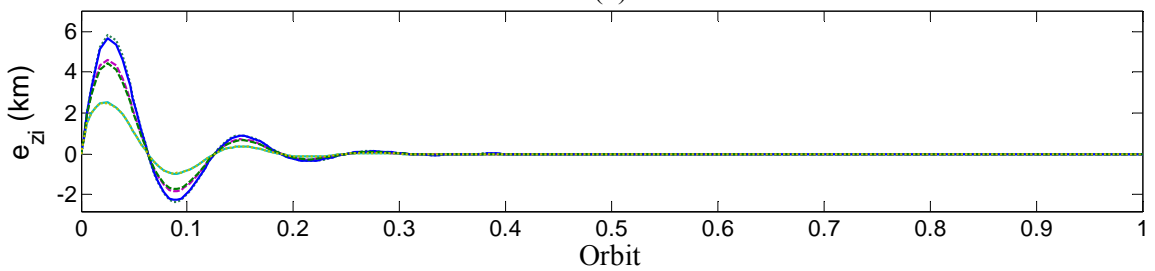

(c)

Figure 3.11: Tracking errors (with $10 \mathrm{mN}$ thruster saturation)
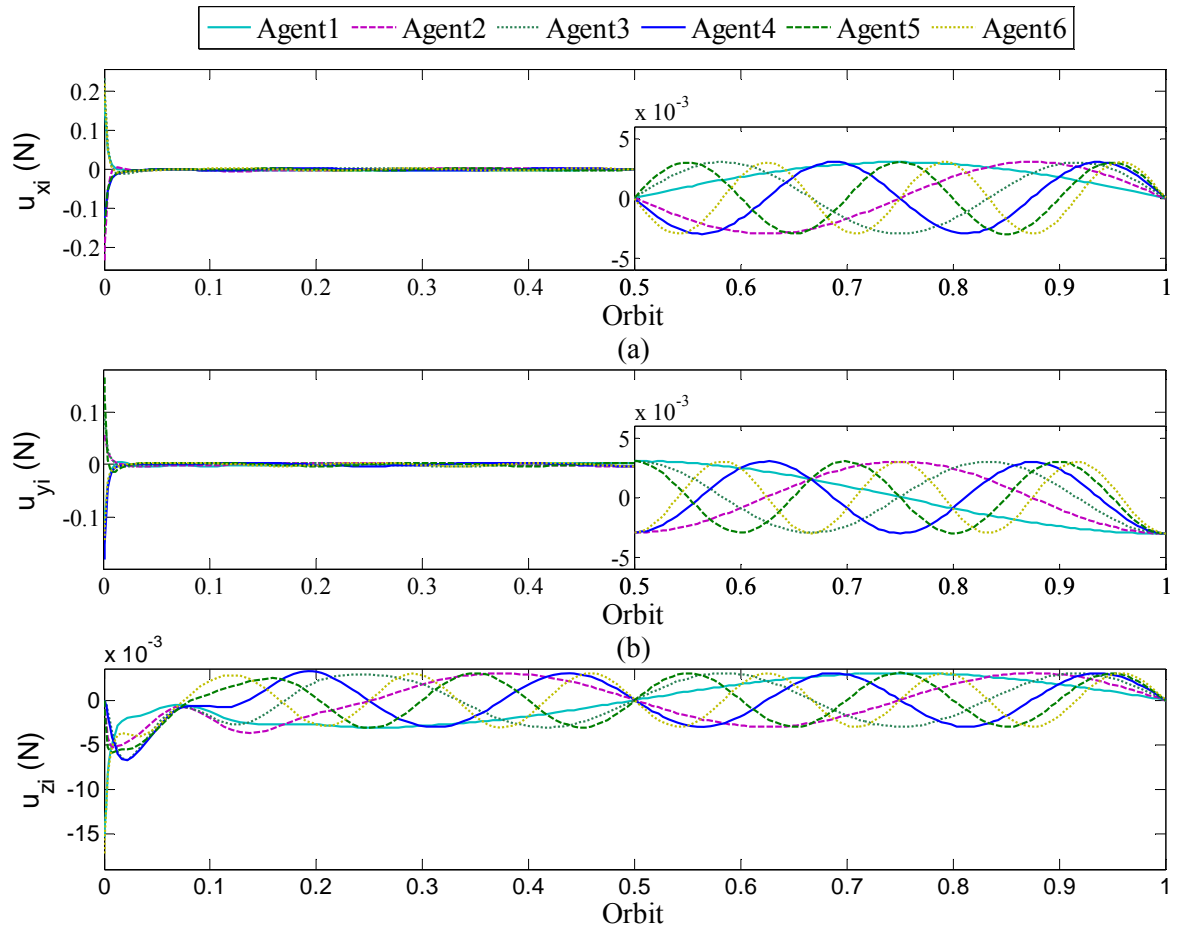

(c)

Figure 3.12: Control input (without thruster saturation) 

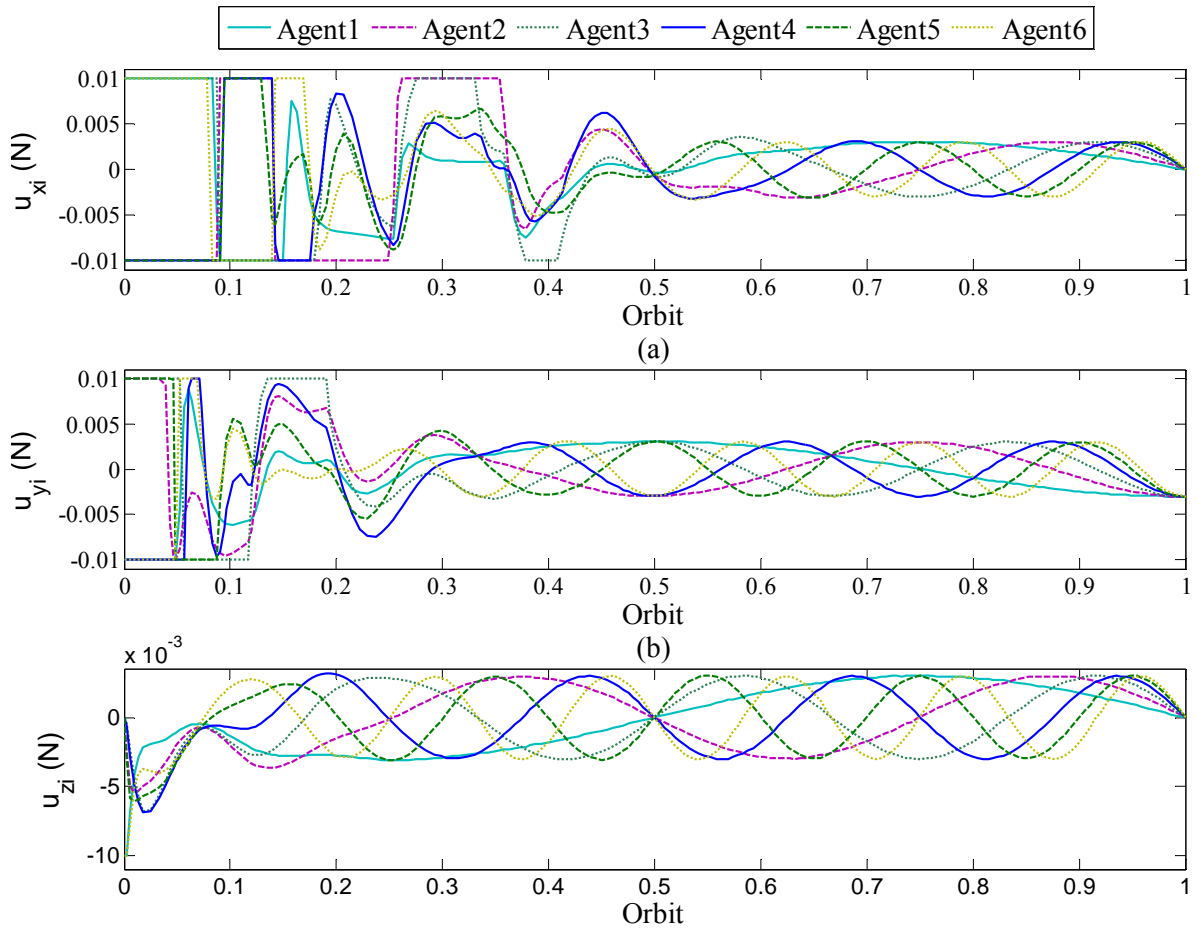

(c)

Figure 3.13: Control input (with $10 \mathrm{mN}$ thruster saturation)

Without changing the control parameters, when there is an insufficient amount of control force supplied to the system, the system might take a longer time to settle, or worse, it will lose control. When the set 1 initial state error is examined with the $5 \mathrm{mN}$ thruster saturation, the worst case scenario occurs. I.e. loss of system control. In Figure 3.14, the thrust required in the xdirection for agents 1 to 4 stays at a maximum for most of the simulation time. The control law proposed is able to control y and $\mathrm{z}$ direction after the initial state error, see Figure 3.15. However, due to the coupled effects of the dynamic system in the $\mathrm{x}$ and $\mathrm{y}$ directions, when $\mathrm{x}$ diverges, $\mathrm{y}$ follows after the third orbit, Figure 3.16, while z remains controlled. 

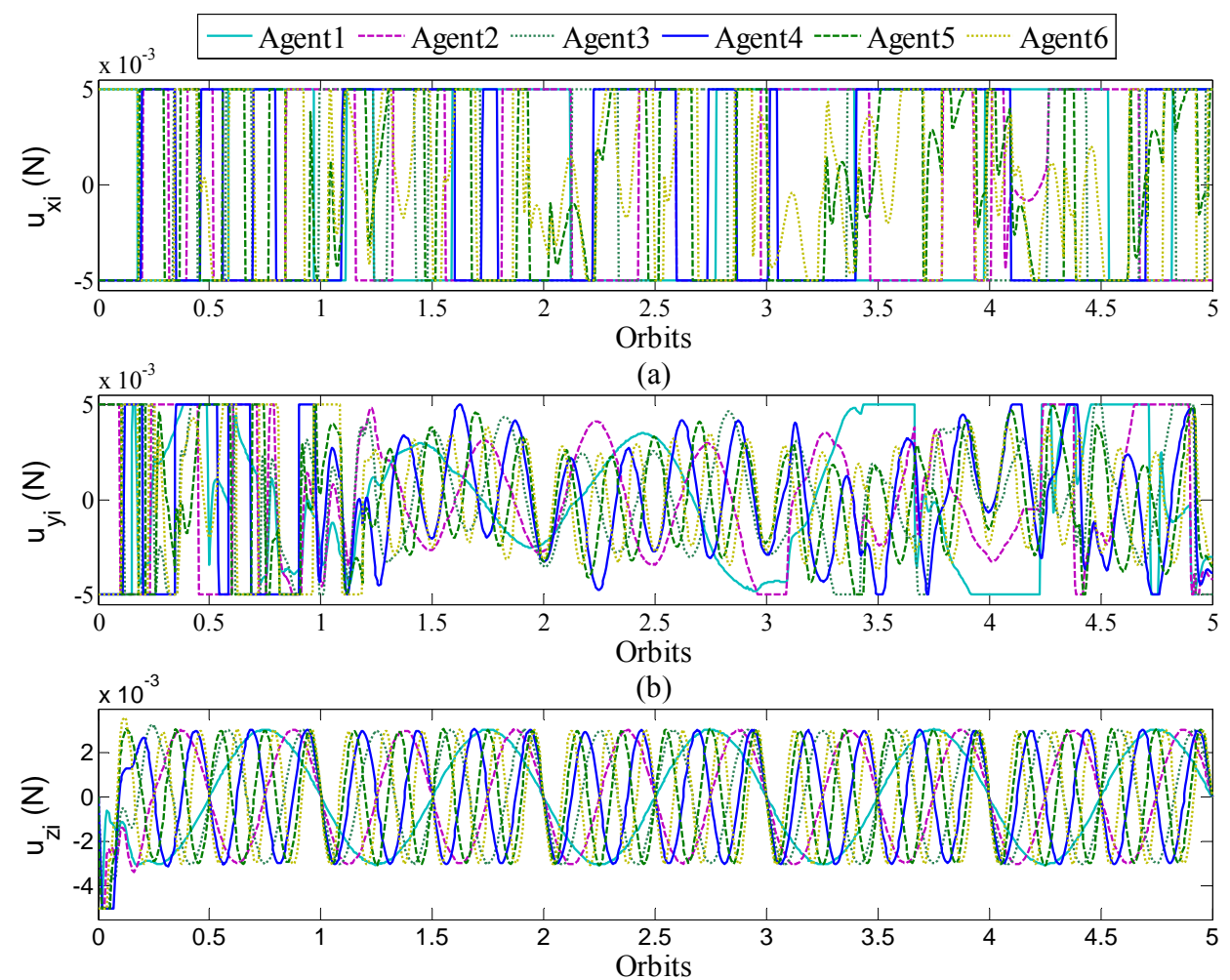

(c)

Figure 3.14: Control input (with $5 \mathrm{mN}$ thruster saturation)

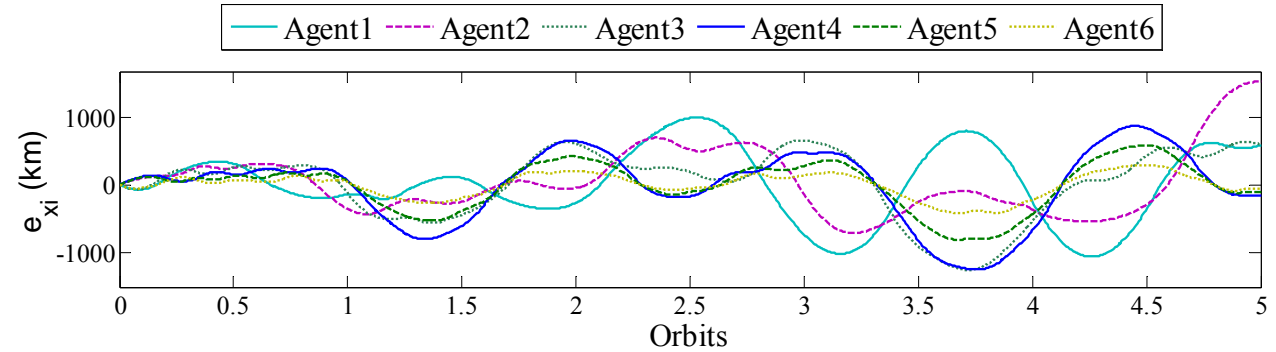

(a)

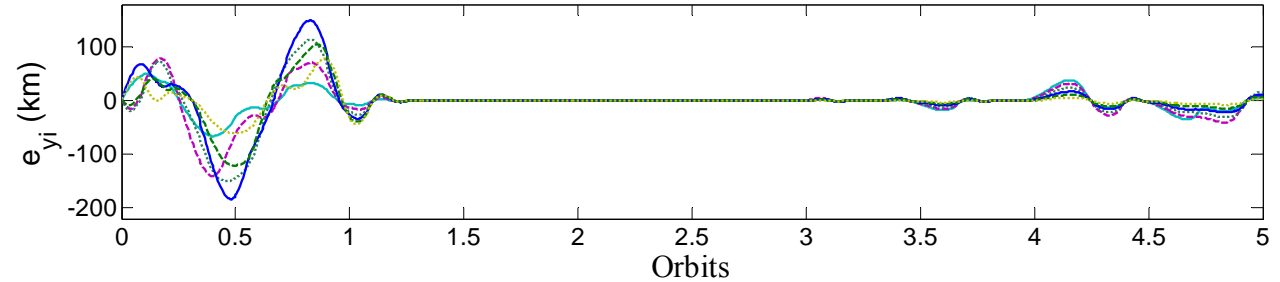

(b)

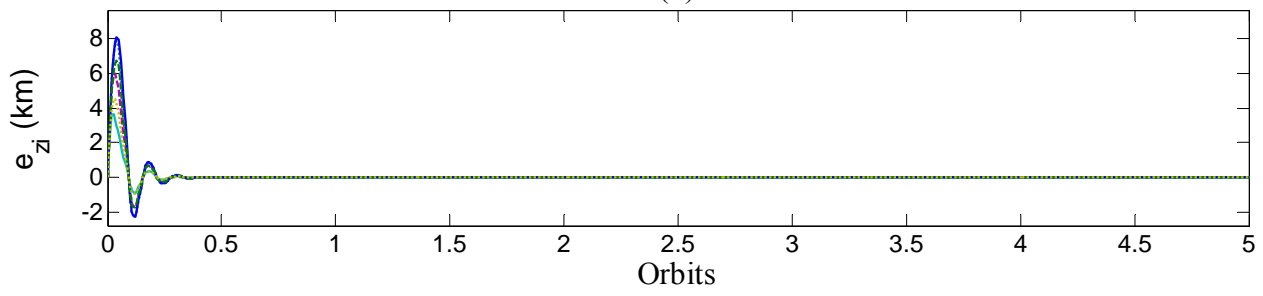

(c)

Figure 3.15: Tracking errors (with $5 \mathrm{mN}$ thruster saturation) 

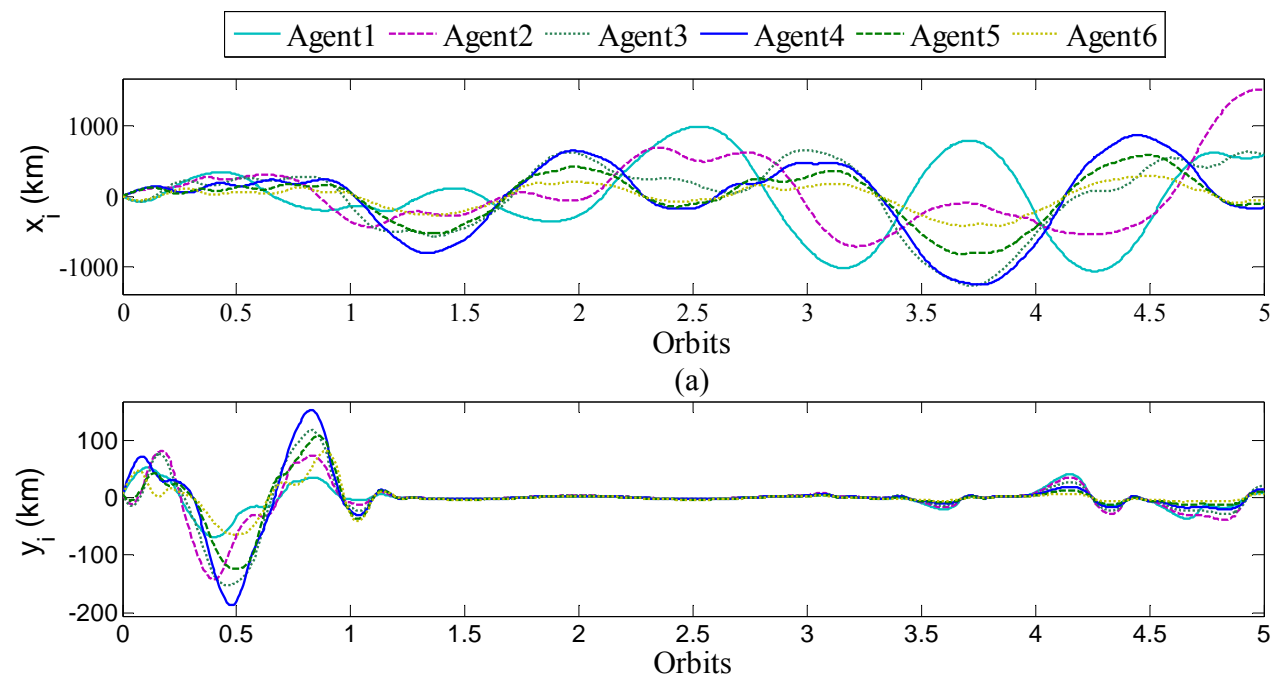

(b)

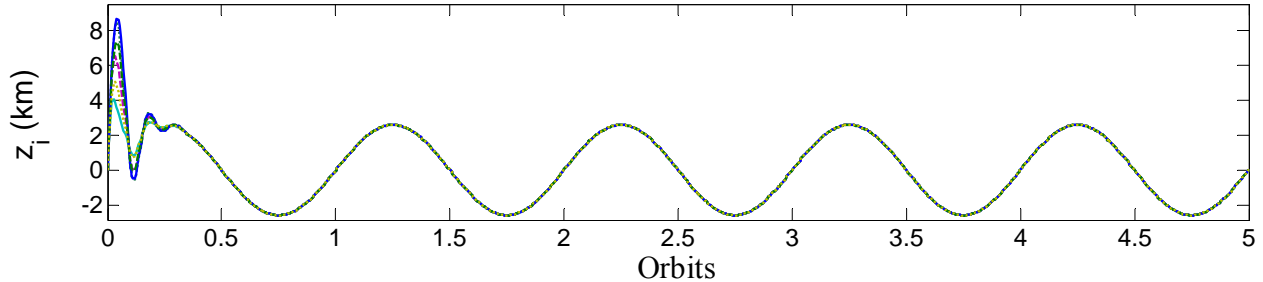

(c)

Figure 3.16: State of all agents (with $5 \mathrm{mN}$ thruster saturation)

With the introduction of the disturbance to the system, the control law proposed is able to control the system even with the set 1 initial state error. However, when there is only $5 \mathrm{mN}$ thrust available to the system, with $60 \%$ of the maximum actuator force disturbance, the control law fell short in bringing all 6 agents back to the reference trajectory. These cases are further investigated in Chapter 4.

\subsubsection{Effect of Sensors Faults}

To investigate the response of the spacecraft experiencing sensor faults, some baseline results are generated through simulation, Figure 3.17 to Figure 3.21. Set 2 initial state error is used, and disturbances are not applied; results shown are purely caused by sensors faults. Two specific types of sensor faults are modeled, the sensor gives no signal or the right value but wrong sign. 


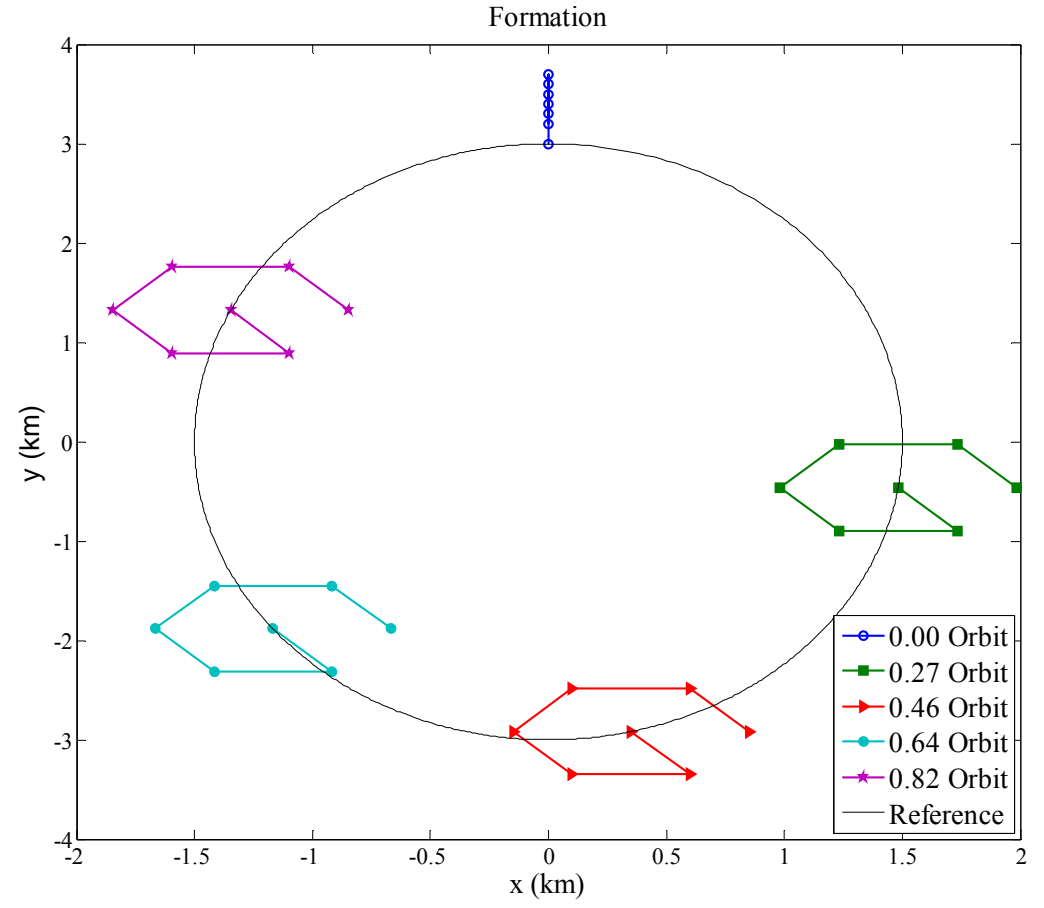

Figure 3.17: System state snap shot at different time (without thruster saturation)
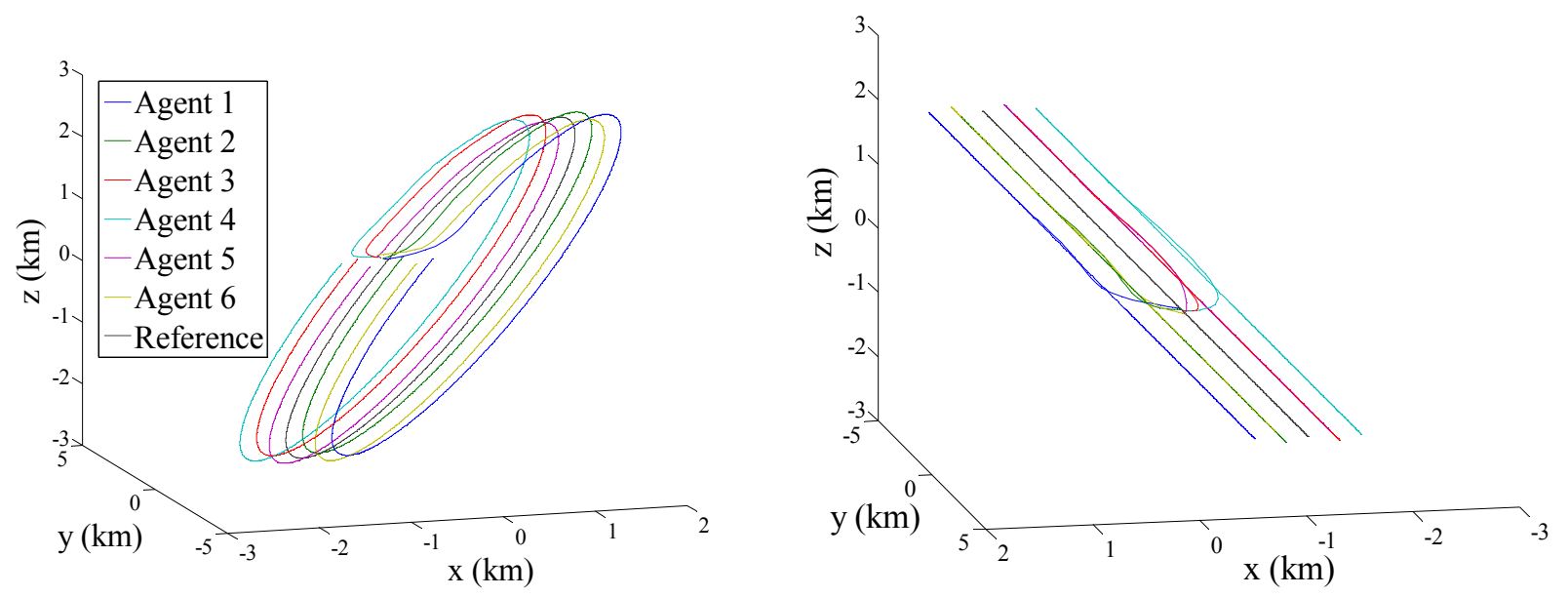

Figure 3.18: Two 3D views of system state (without thruster saturation) 


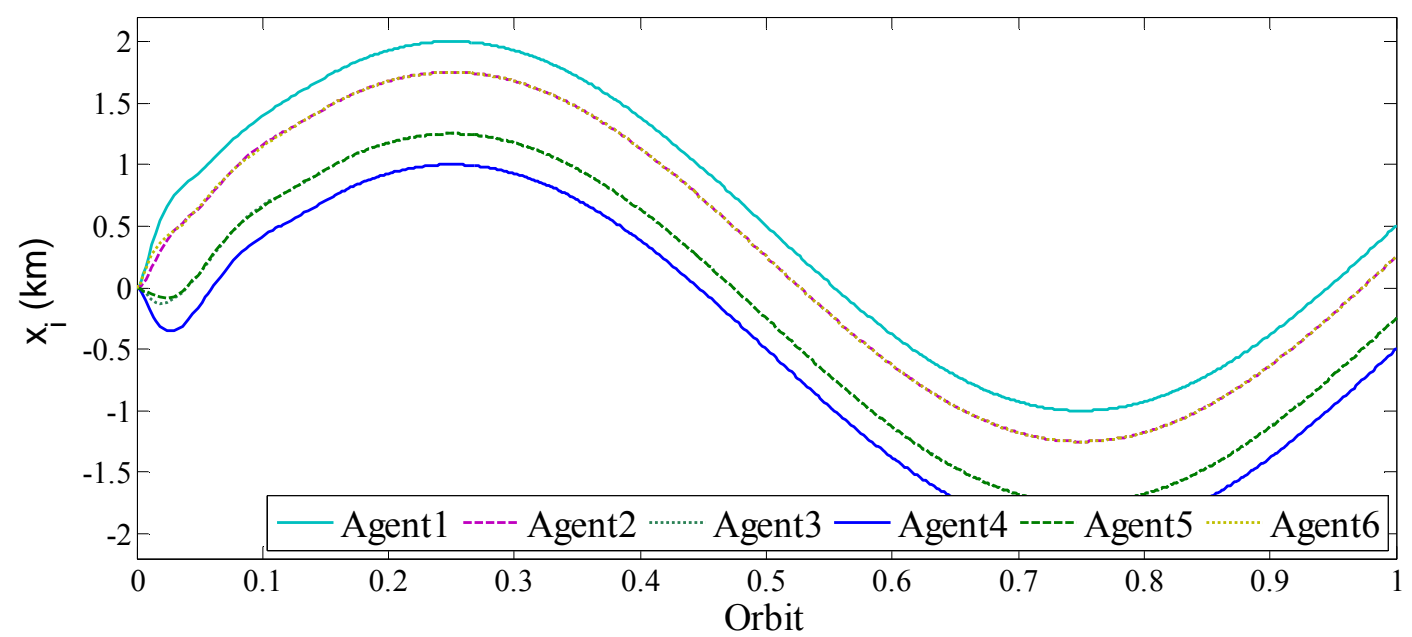

(a)

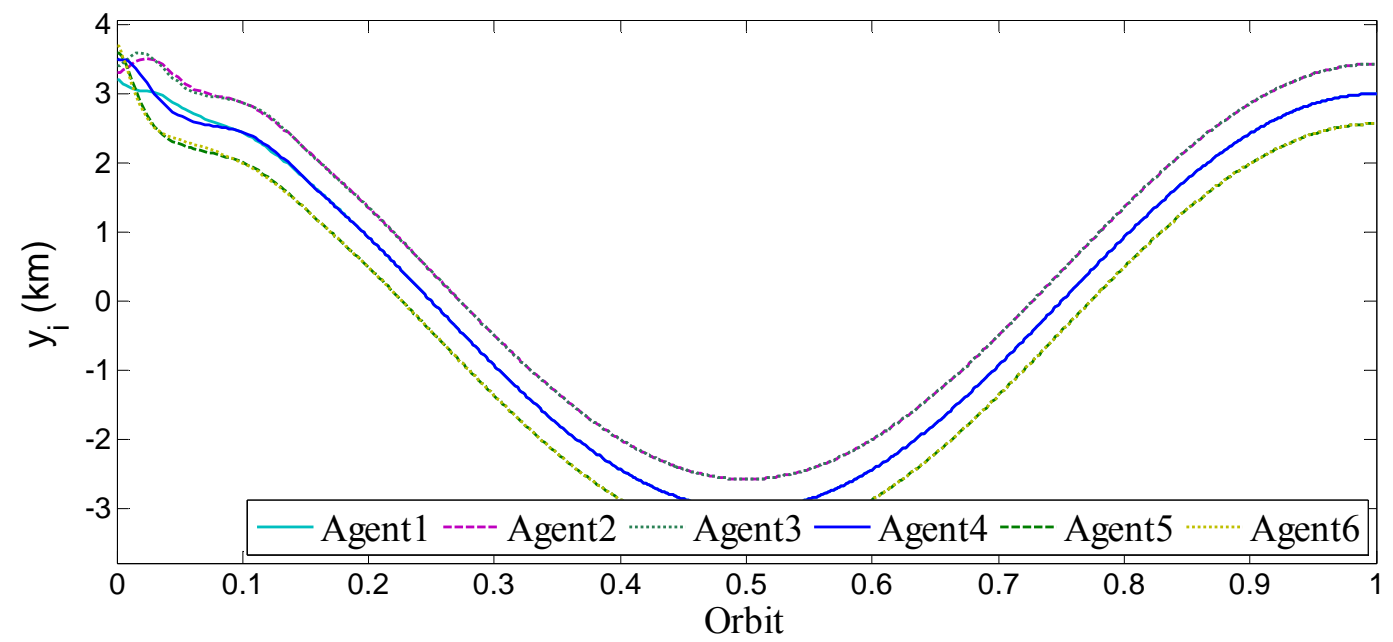

(b)

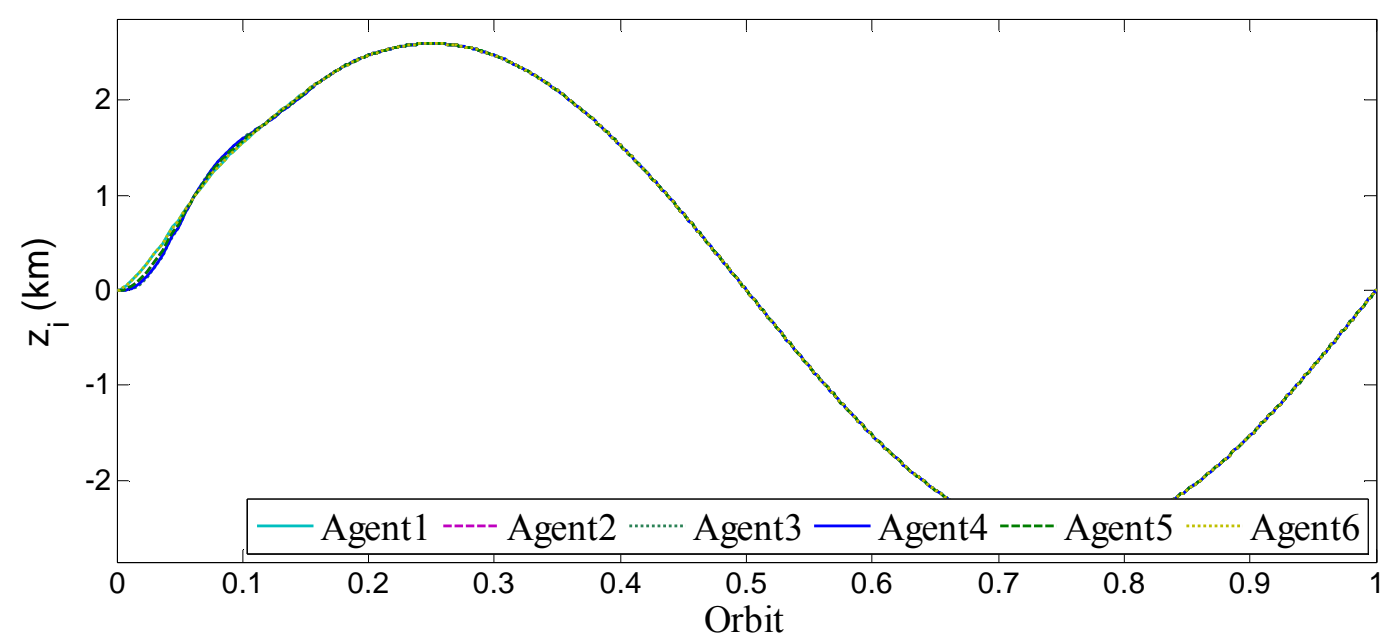

(c)

Figure 3.19: State of all agents (without thruster saturation) 

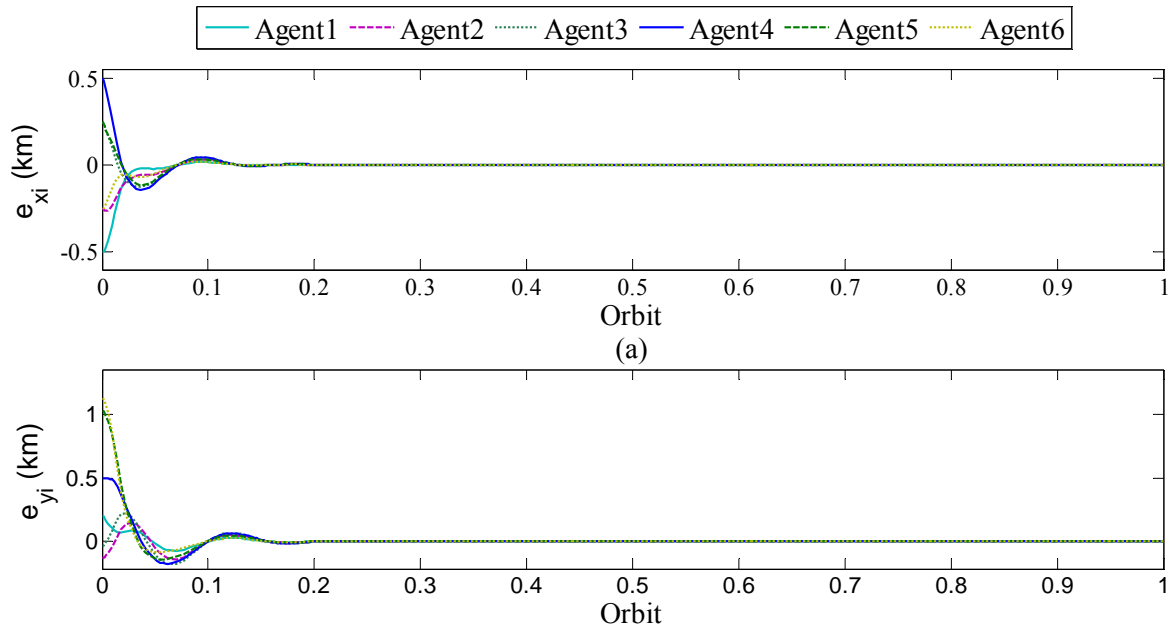

(b)

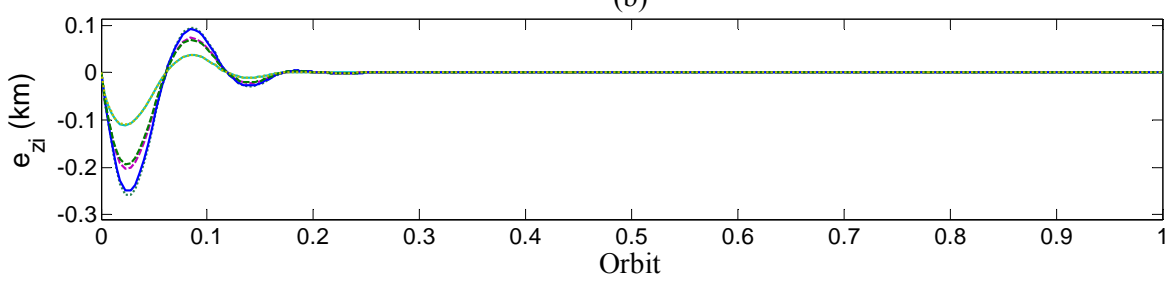

(c)

Figure 3.20: Tracking errors (without thruster saturation)
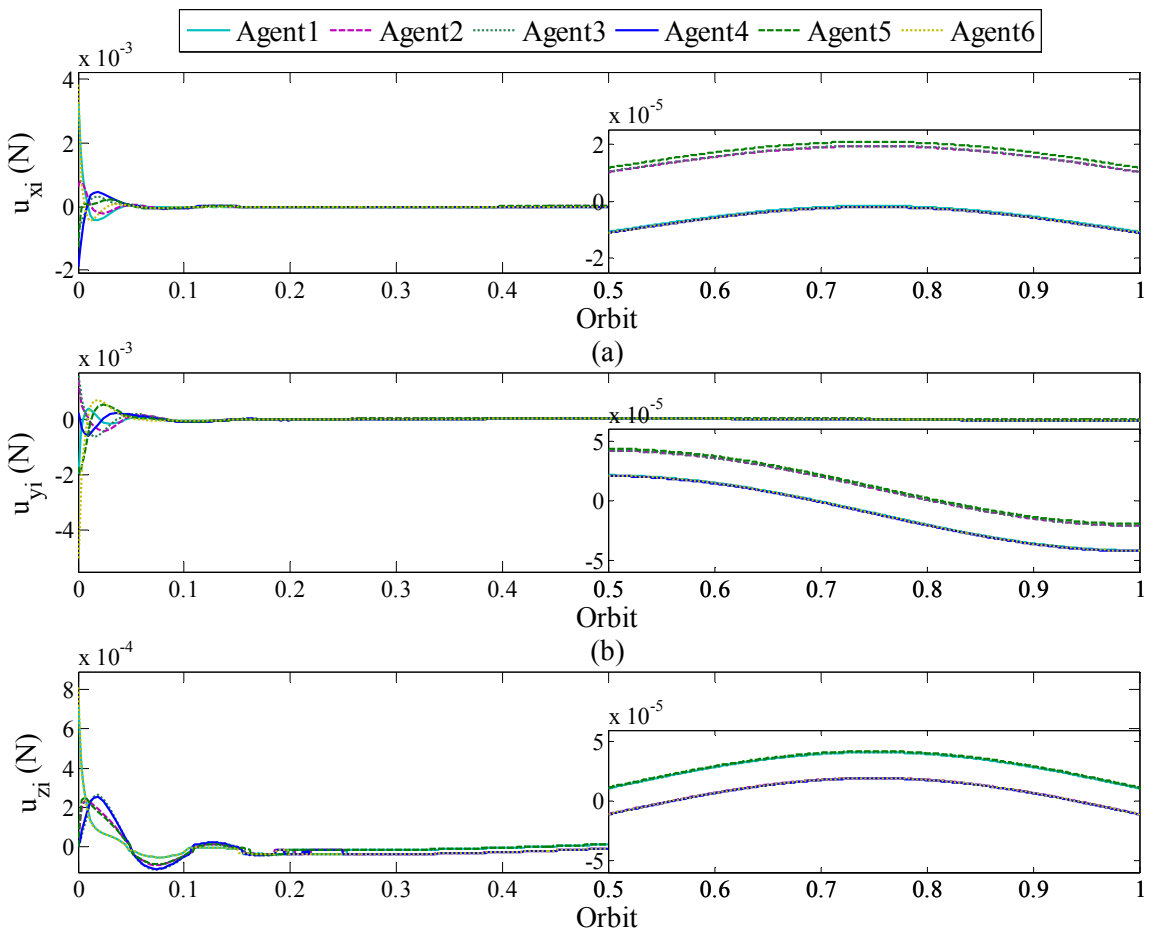

(c)

Figure 3.21: Control input for all agents (without thruster saturation) 
In Figure 3.17, it is clear that set 2 initial state error is applied, and the system reaches formation consensus in less than 0.27 of an orbit. Two 3D views in Figure 3.18 show that even when there are lines crossing in one graph, there are no collisions between agents. Each agent is moving in its own plane as defined in Figure 3.2. It is shown more clearly that due to the defined formation configuration, some lines are crossing and even overlapping one another. In Figure 3.19 (a), the lines represent agents 2 and 6, and agents 3 and 5 are overlapping. In Figure 3.19 (b), the lines representing agents 1 and 4, agents 2 and 6 , and agents 3 and 5 are overlapping, and all agents have the same $\mathrm{z}$ value as reflected in Figure 3.19 (c). With the proposed control law, for this specific case, the system is controlled, shown in Figure 3.20, and the control efforts are less than $5 \mathrm{mN}$, as seen in Figure 3.21.

\subsubsection{Sensor no signal}

Both types of previously mentioned sensor faults are examined here, and it is assumed that the sensors along all the $\mathrm{x}, \mathrm{y}$ and $\mathrm{z}$ directions are defective. The first case being investigated is the sensor giving a zero value. This scenario is examined with only one agent experiencing the sensor downtime, three agents experiencing the sensor downtime, and all six agents experiencing the downtime for ten minutes. As previously mentioned, 1 reference orbit is approximately 0.5087 hour, therefore, the duration of the downtime is roughly one third of an orbit. The sensors downtime is set to start at 400 seconds and end at 1000 seconds, which is 0.2184 and 0.5460 of an orbit, respectively.

In Figure 3.20, tracking errors have converged to zero after one fifth of an orbit, which means the system is controlled. That is the reason that the downtime is introduced at 0.2184 of an orbit. Due to the consensus algorithm, both tracking error and relative error are monitored and controlled by the proposed control law. This means that the control topology is successful in controlling the formation and the trajectory at the same time. Figure 3.22 shows when agent 1's sensor is giving a zero value from 0.2184 to 0.5460 of an orbit. The error goes from zero at 0.2184 of an orbit and increases until 0.5460 of an orbit. When the sensor fault is corrected, then the system state returns to the desired state. 


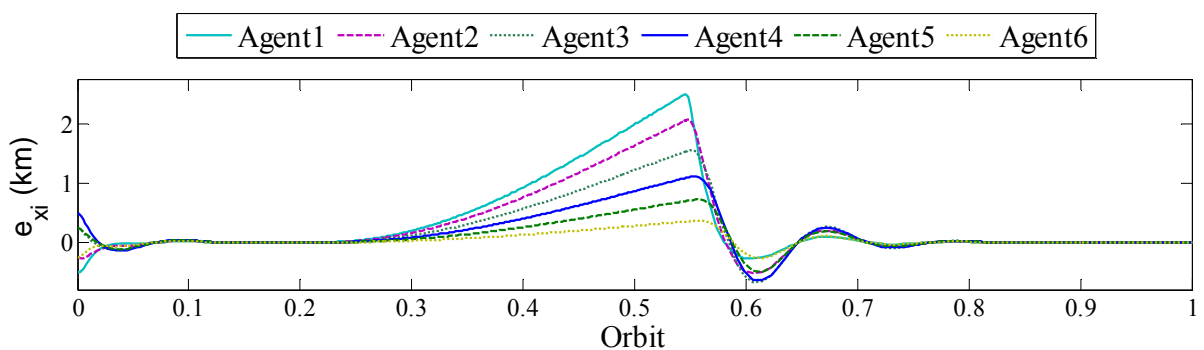

(a)

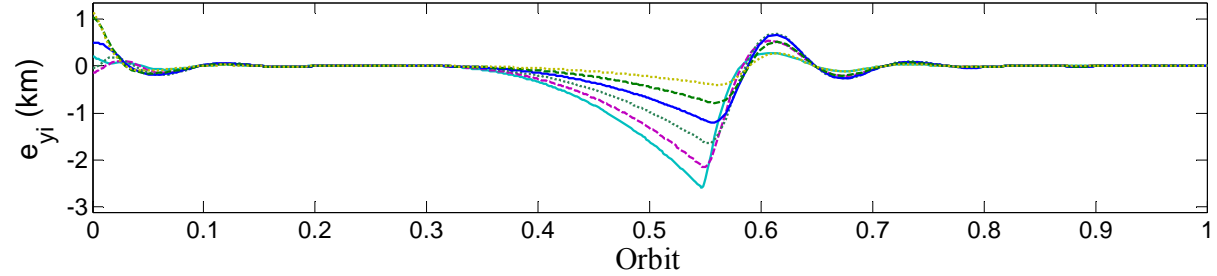

(b)

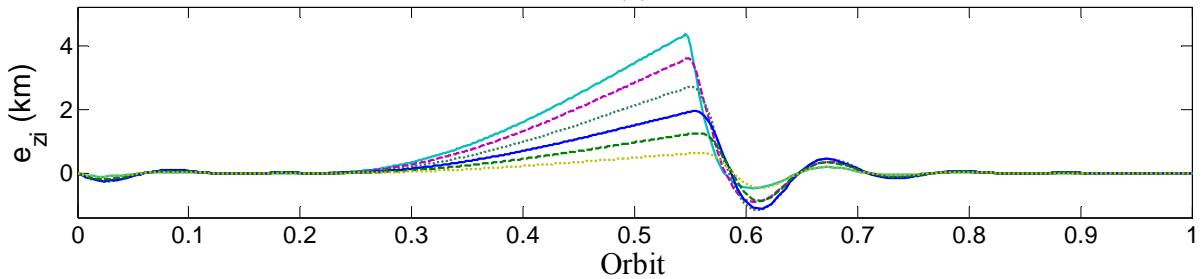

(c)

Figure 3.22: Tracking errors, agent 1's sensor malfunction

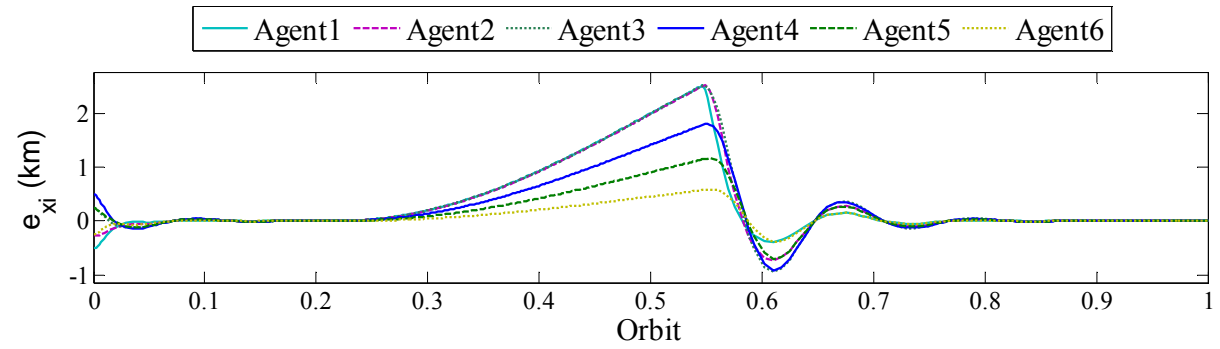

(a)

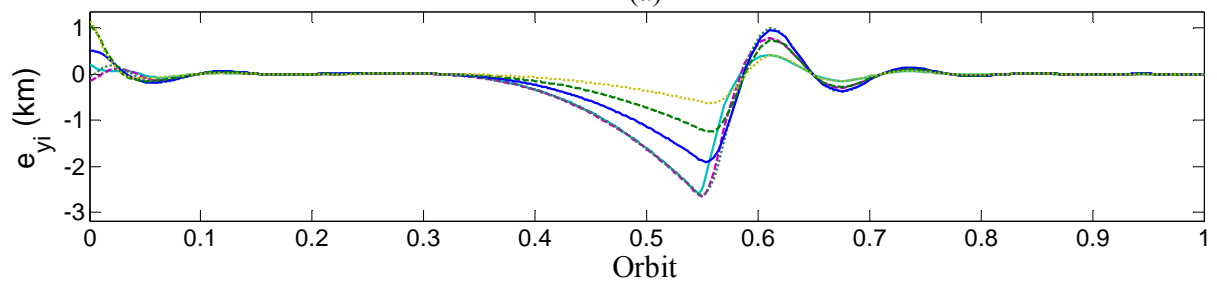

(b)

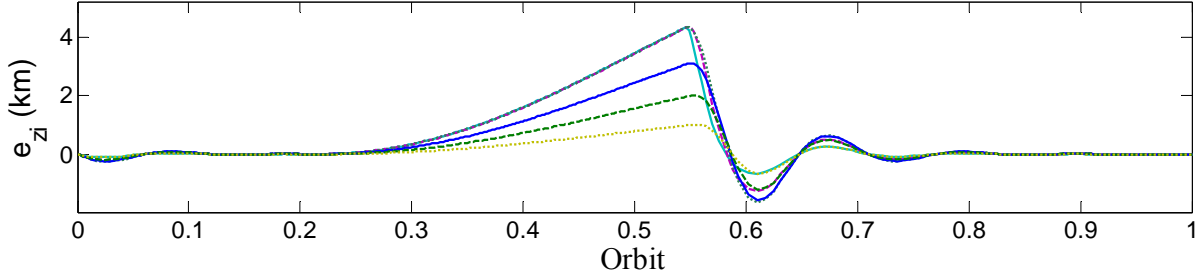

(c)

Figure 3.23: Tracking errors, agent 1, 2 and 3's sensor malfunction 
It is important to mention that, in both cases, Figure 3.22 and Figure 3.23, when agent 1's sensors are giving a zero value, agent 2's tracking errors have increased the most when compared with agents 3, 4, 5 and 6 , with agent 6 being the least affected. When the sensor on agents 1, 2, and 3 are not working, agent 4 is being affected the most, 5 and 6 the least. This is governed by the communication link specified in Equation (3.42) in which, agents 1 and 2 have a directed link, but agents 1 and 6 have no direct communication.

All 6 agents are experiencing the same sensor fault, and the errors are the same. However, the required system control input, seen in Figure 3.24, is different due to the position of the agents compared with the reference trajectory. Agents 1 and 6 certainly require increased thrust when experiencing this sensor fault. Figure 3.25 shows the control input with $5 \mathrm{mN}$ saturation when all 6 agents are experiencing sensor faults, while Figure 3.26 displays when the proposed control law is successful in tracking the desired trajectory.
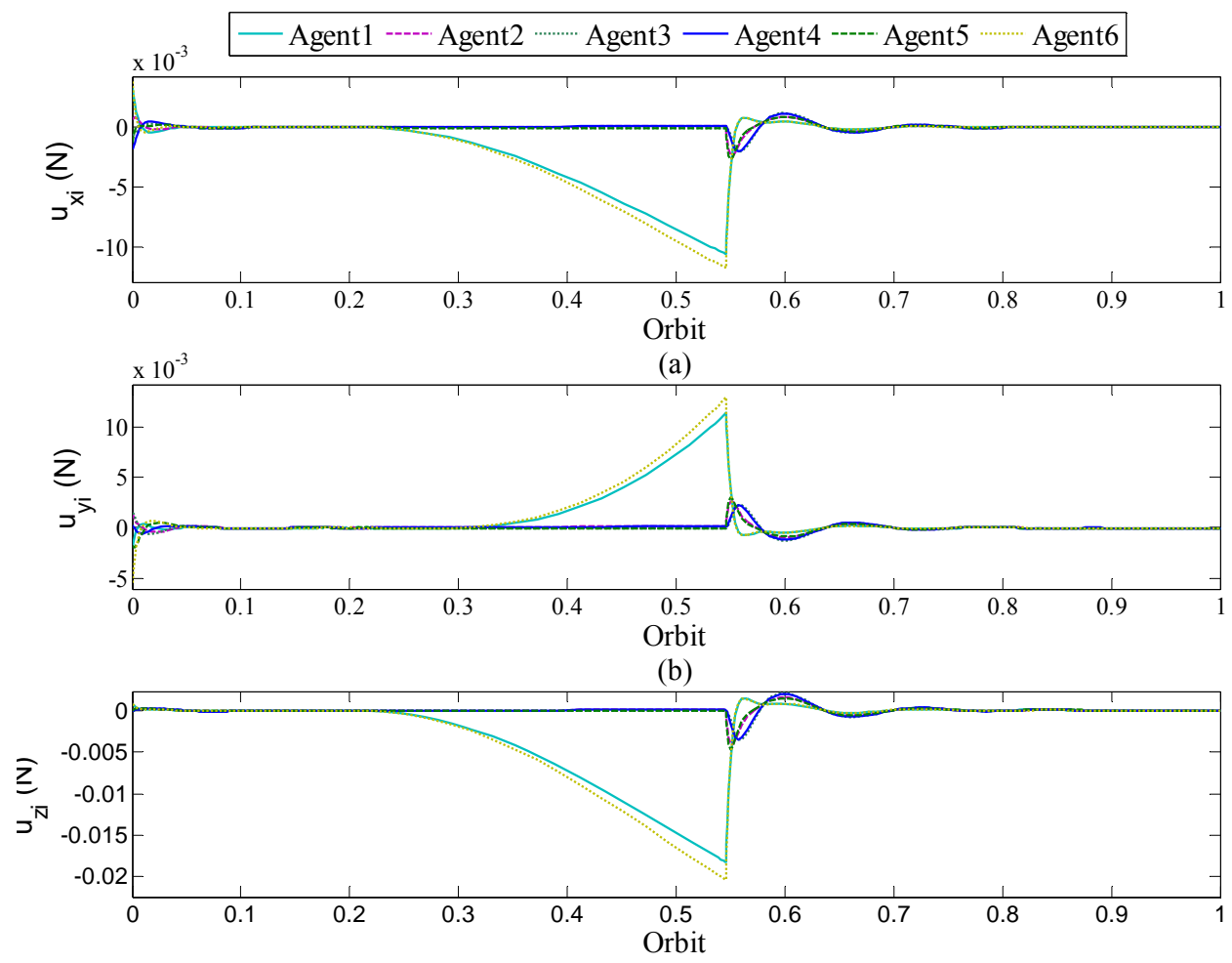

(c)

Figure 3.24: Control input, all six agents' sensor malfunction 

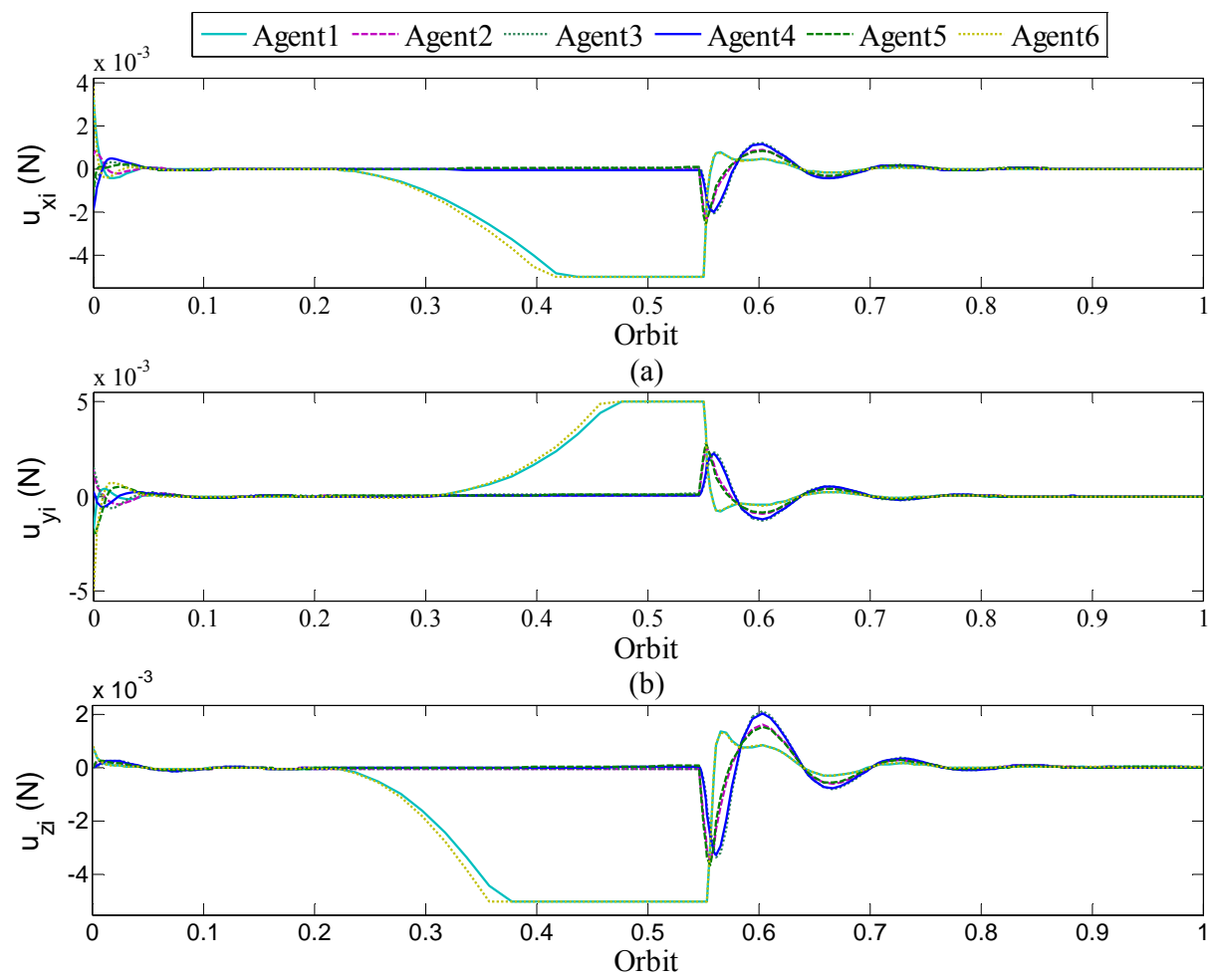

(c)

Figure 3.25: Control input, all six agents' sensor malfunction (with $5 \mathrm{mN}$ saturation)
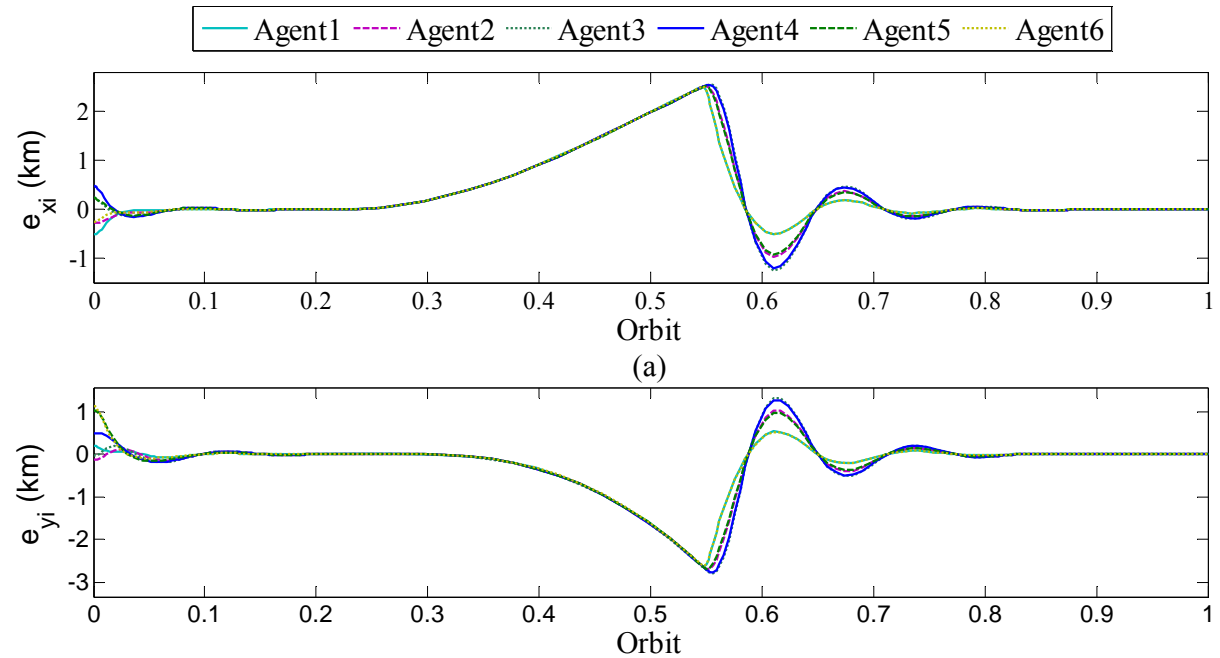

(b)

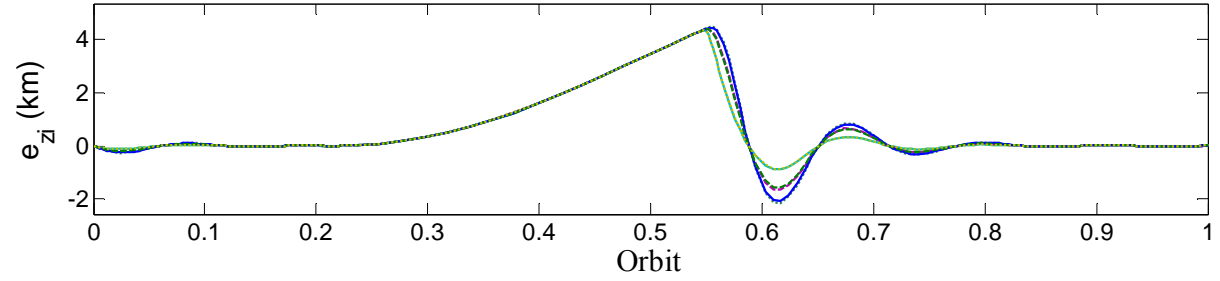

(c)

Figure 3.26: Tracking errors, all agents' sensor malfunction (with $5 \mathrm{mN}$ saturation) 
In real missions, it is known that when faults occur in the sensors, they might not be repaired in the short duration. Here, a 10 minute duration is chosen in order to follow the mission requirements. If the limitation of recovering within 1 orbit does not exist, the duration of the downtime can be extended. Whether or not the proposed control law is capable of bringing the agents back to the desired trajectory after the downtime is not just dependent on the duration of the downtime. This is also a function of the available actuator force from the thruster, and the state of the spacecraft. However, this gives a good inspection window for this particular mission. If one, or more than one agent is off track for 10 minutes, a decision should be made to remove it from the system to avoid further performance deterioration of the other agents. This is because, as seen in the first scenario, Figure 3.22, when agent 1 is off track, in order to maintain the swarm formation, other agents will follow agent 1 to compensate for the relative error. If agent 1 has not recovered from the sensor fault, and if it has not been removed from the swarm, this small defect may lead to the mission failure.

\subsubsection{Sensor signal interference (sign reversed)}

The duration of the downtime the system can handle depends on the control system and the type of the faults. Continuing with the same control law proposed, using the same control parameters, and following the same mission restrictions, the sensor interference will only last for 2 minutes, and it is applied to the system between 0.2184 and 0.2839 of an orbit. This section shows that detecting the sensor state signal in the wrong direction is a more serious problem than having no signal from the sensors. The first case shown is one agent with sensor fault; in the second, three agents experience the same fault with a thruster saturation; and last, all 6 agents' sensors are giving signals with reversed signs.

Figure 3.27 depicts a similar pattern. Where the sensor is experiencing no signal, other agents are affected when only one agent's sensors malfunction. Figure 3.28 clearly shows the effects of consensus formation tracking; when every other agents' sensor in the communication system is defective, all agents are affected. Agent 4 experiences the largest effect because the error is contributed both from its own sensors as well as the errors generated from agents 2 and 6 . 

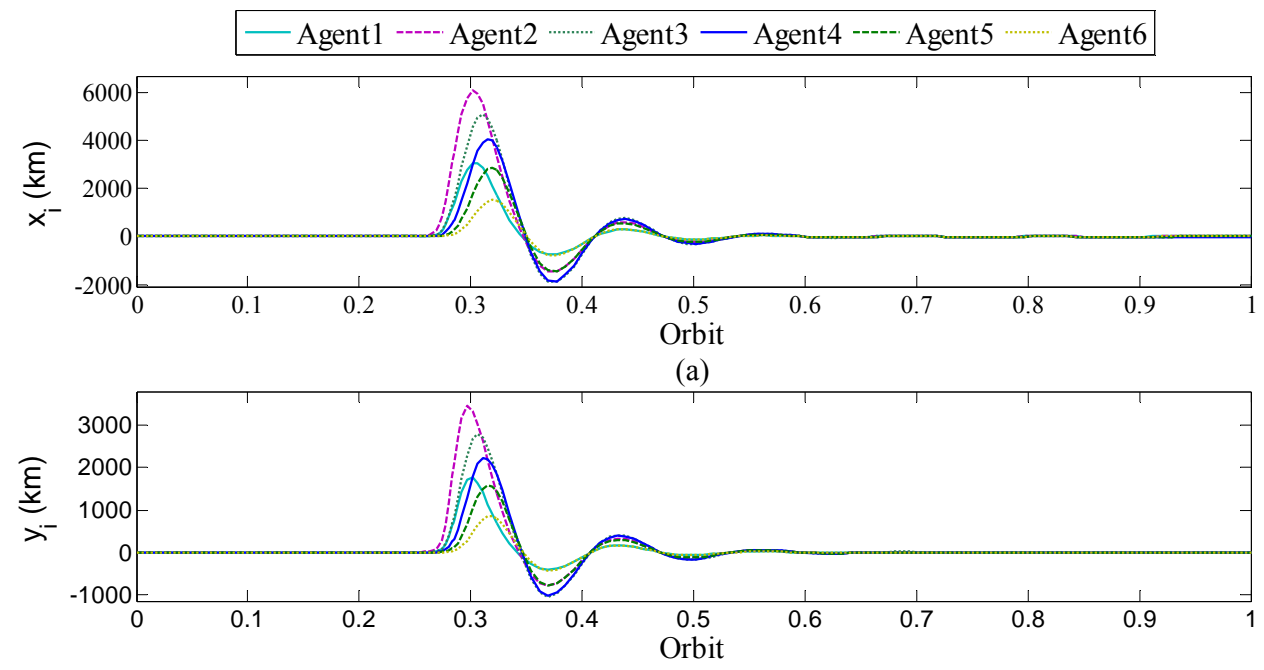

(b)

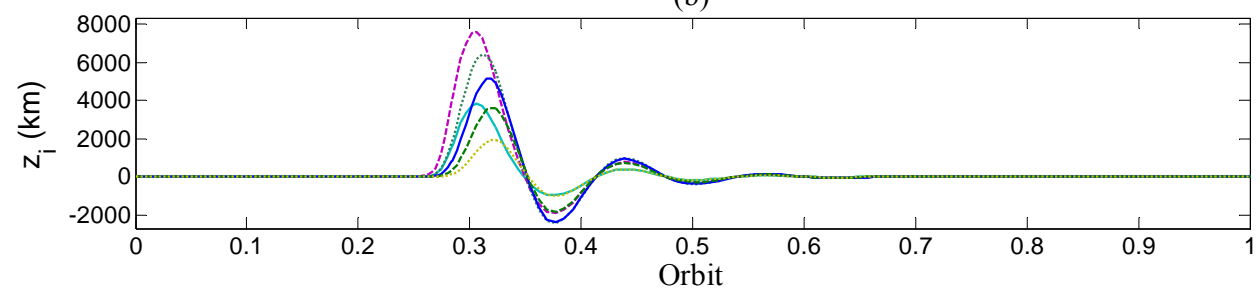

(c)

Figure 3.27: State of all agents, sensor fault in agent 2

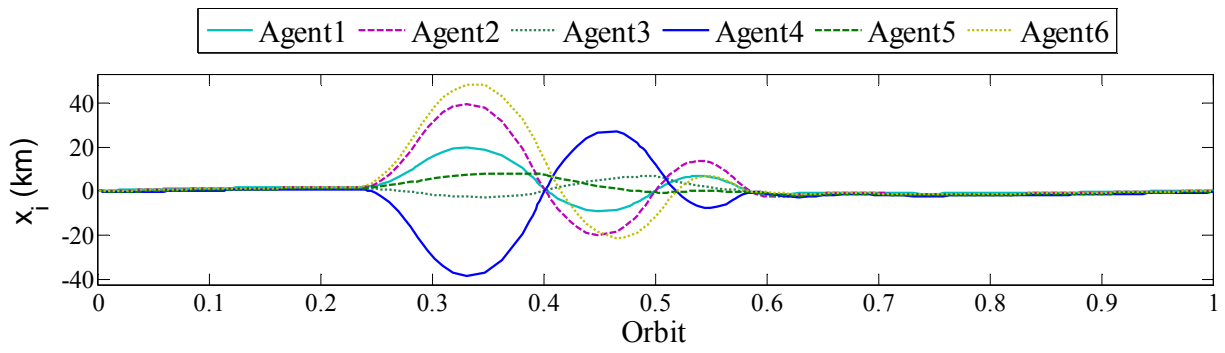

(a)

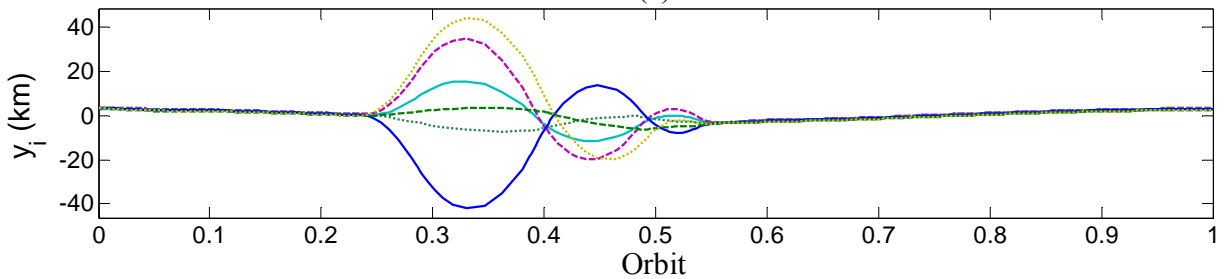

(b)

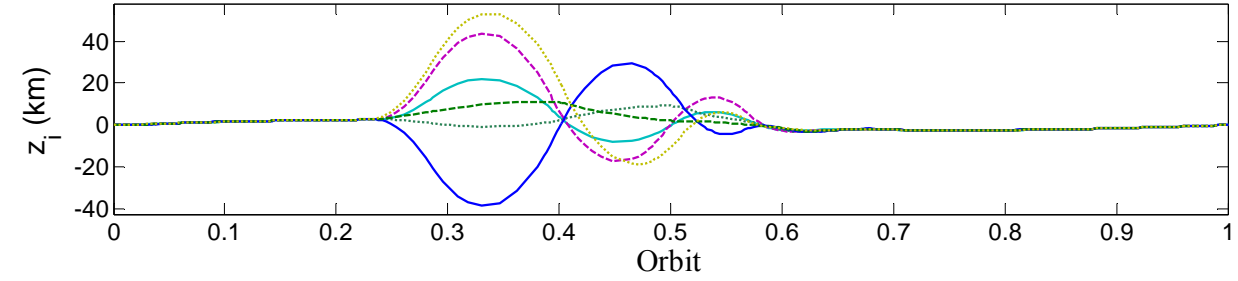

(c)

Figure 3.28: State of all agents, sensor fault in agent 2, 4 and 6 
When the maximum thrust available is set to be $5 \mathrm{mN}$, horizontal lines in Figure 3.29, it also limits the possible state error caused by the sensors fault. As seen in Figure 3.27, the maximum distance reaches almost $8,000 \mathrm{~km}$ away from the asteroid. Because of the assumption that no limitation is applied to the available thrust onboard in the spacecraft, numerically the controller is capable of bringing the spacecraft back to the reference trajectory.
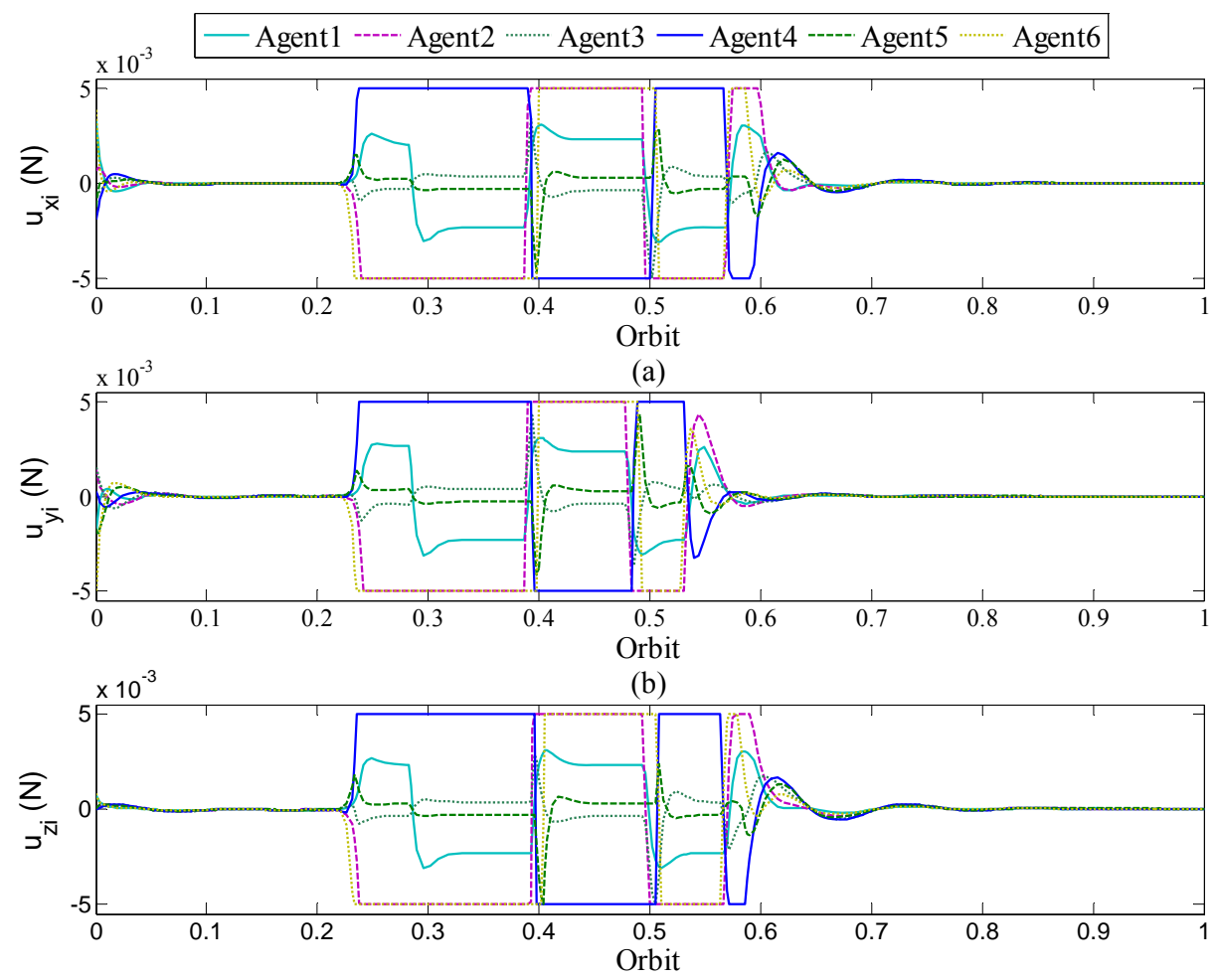

(c)

Figure 3.29: Control input, agent 2, 4 and 6' sensor malfunction (with $5 \mathrm{mN}$ saturation) 

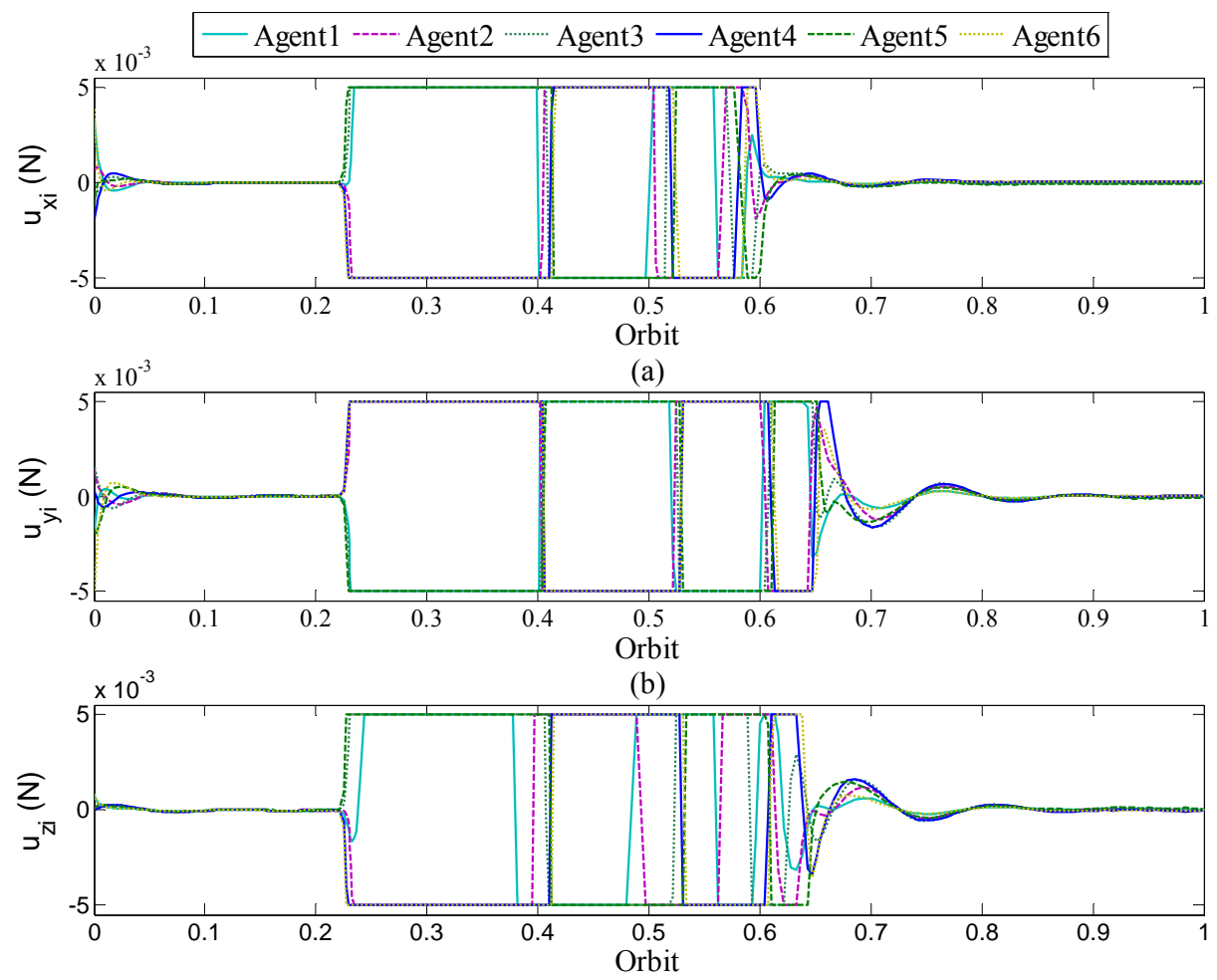

(c)

Figure 3.30: Control input, all six agents' sensor malfunction (with $5 \mathrm{mN}$ saturation)

Even with the application of thrust saturation when all 6 agents' sensors are not working properly, showing in Figure 3.30, the system is controlled within 1 orbit. Figure 3.31 and Figure 3.32 are the tracking errors of the system when there are 3 agents and 6 agents being affected by the sensors' fault. The system is controlled in both scenarios as the error reaches zero within 1 orbit. 


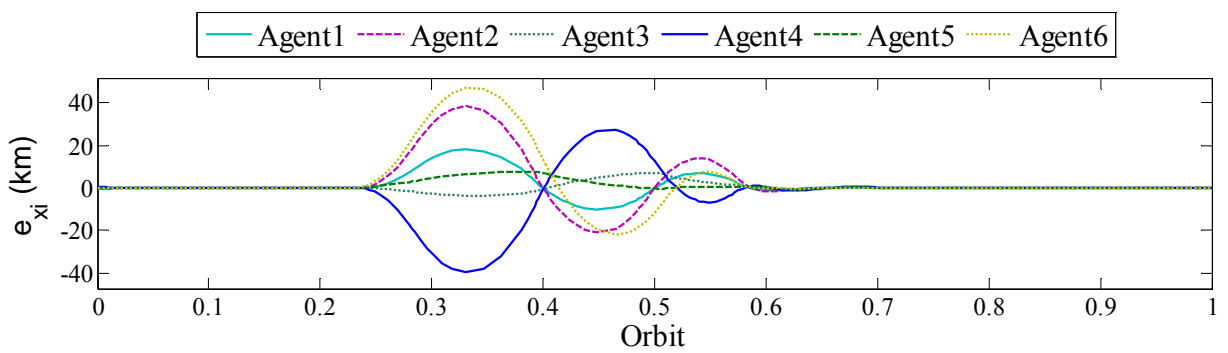

(a)

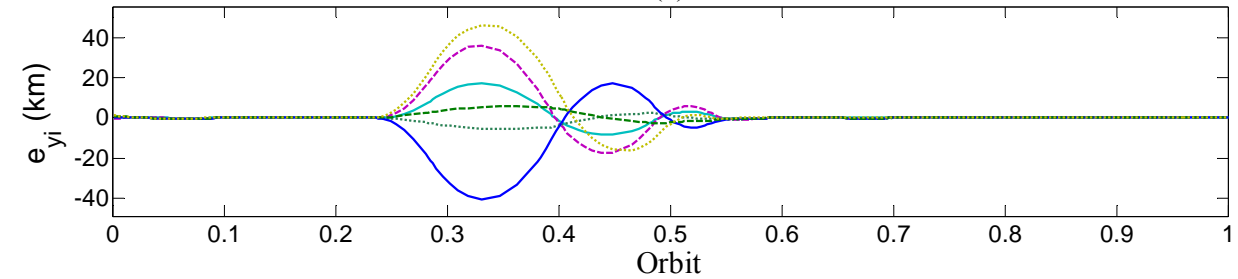

(b)

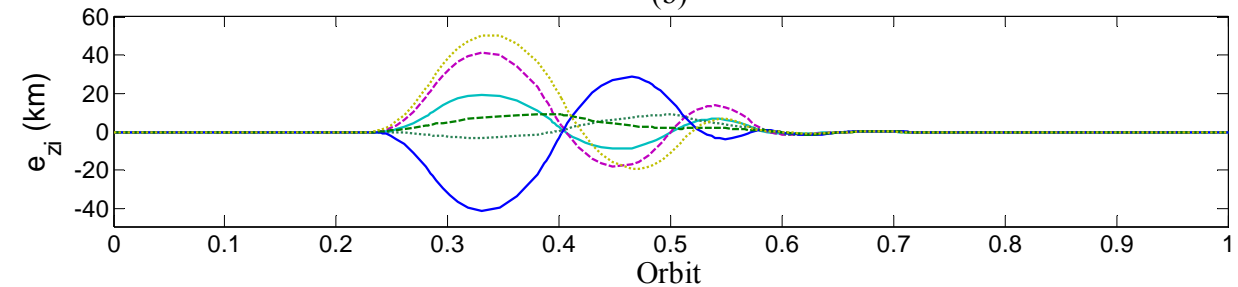

(c)

Figure 3.31: Tracking errors, agent 2, 4, and 6' sensor malfunction (with $5 \mathrm{mN}$ saturation)
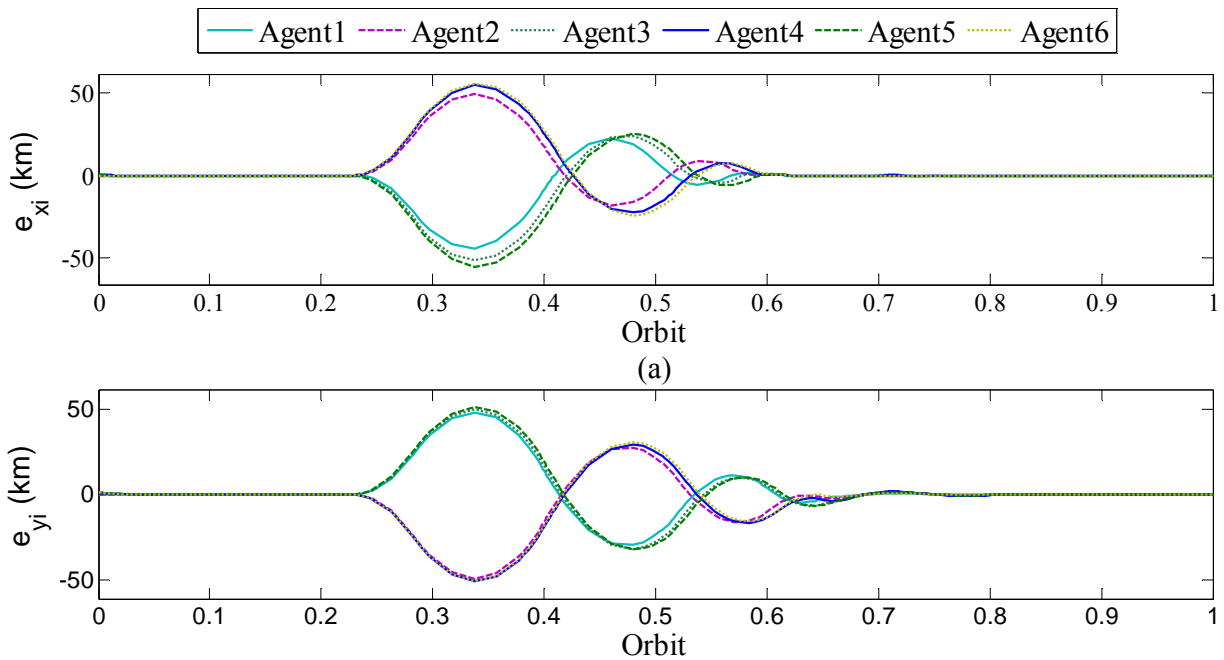

(b)

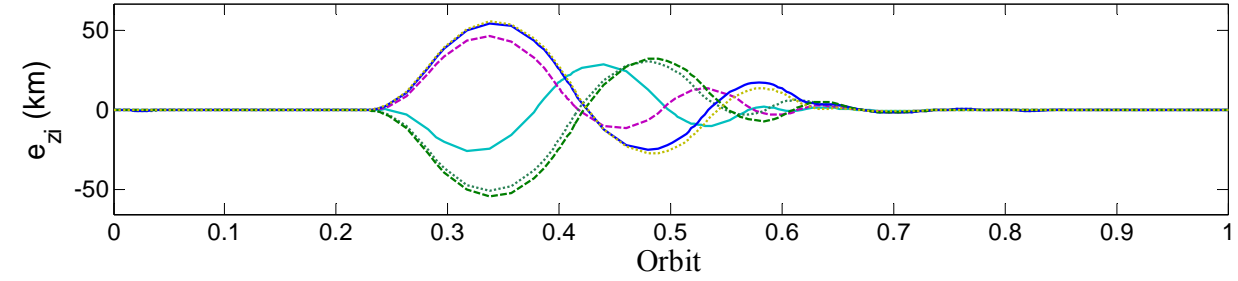

(c)

Figure 3.32: Tracking errors, all agents' sensor malfunction (with $5 \mathrm{mN}$ saturation) 
Through the examination of the results, it is evident that the chaotic level of the state is more controllable when thruster saturation is applied. As predicted, during the downtime, when the amount of thrust is limited, the distance the agents can travel is reduced. This can be verified by comparing Figure 3.27 and Figure 3.28. A sign reverts fault is harder to control than one caused when the sensor has no signal. When system downtime is extended for more than 2 minutes the system will be uncontrollable. However, if more thrust is available, this downtime duration will also increase. As mentioned previously, the duration of the downtime can be treated as the monitor time bracket. If the system is responding in a similar situation, a decision has to be made to avoid mission failure.

\subsubsection{Effect of Spacecraft Mass Variations}

The dynamic equations used to model agents orbiting around slowly rotating asteroids assume the mass of the spacecraft as unity. The following cases are simulated in order to investigate whether or not the proposed control law is capable of controlling the system when the mass of the spacecraft changes.
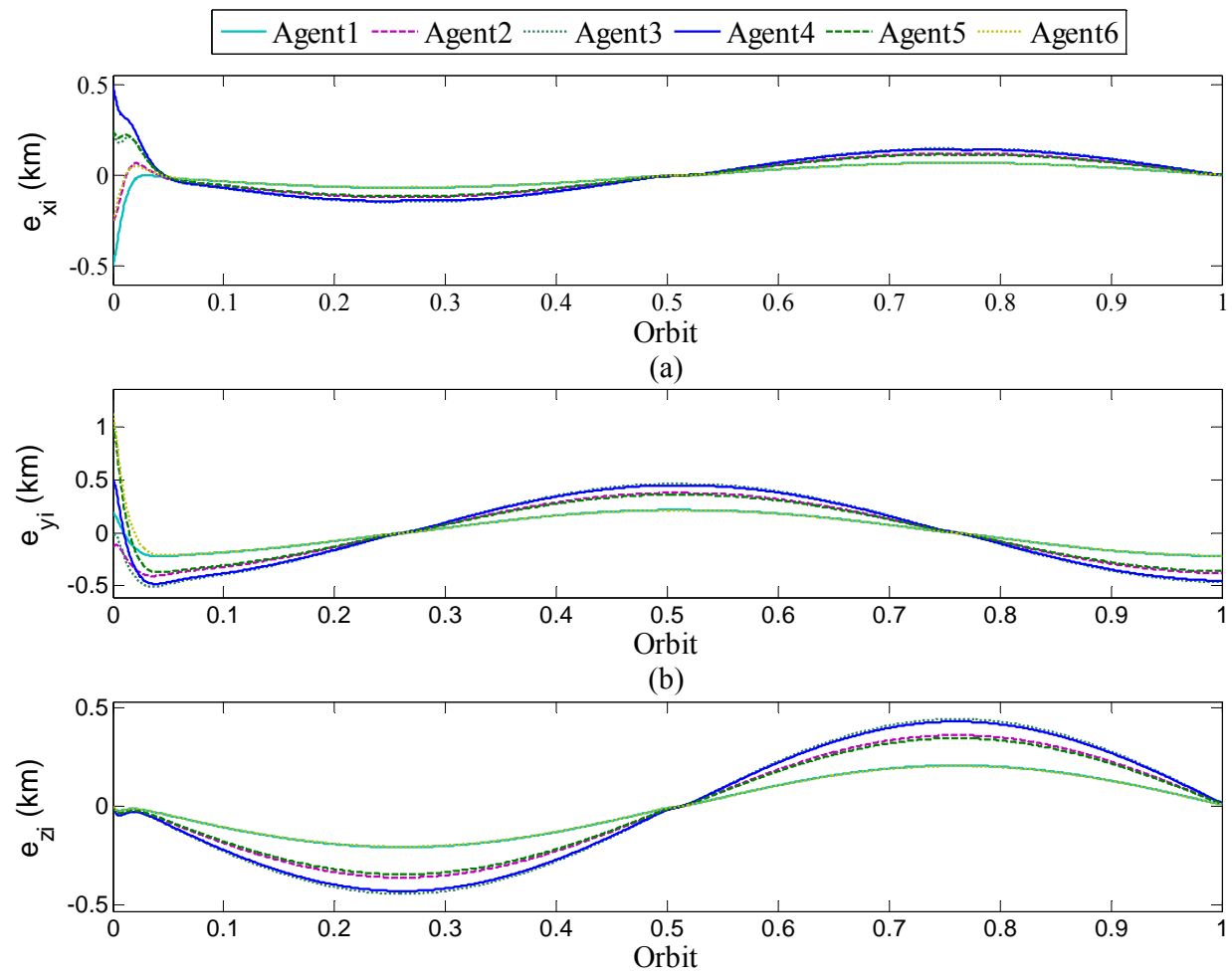

(c)

Figure 3.33: Tracking errors, spacecraft mass $=10 \mathrm{~kg}$ 

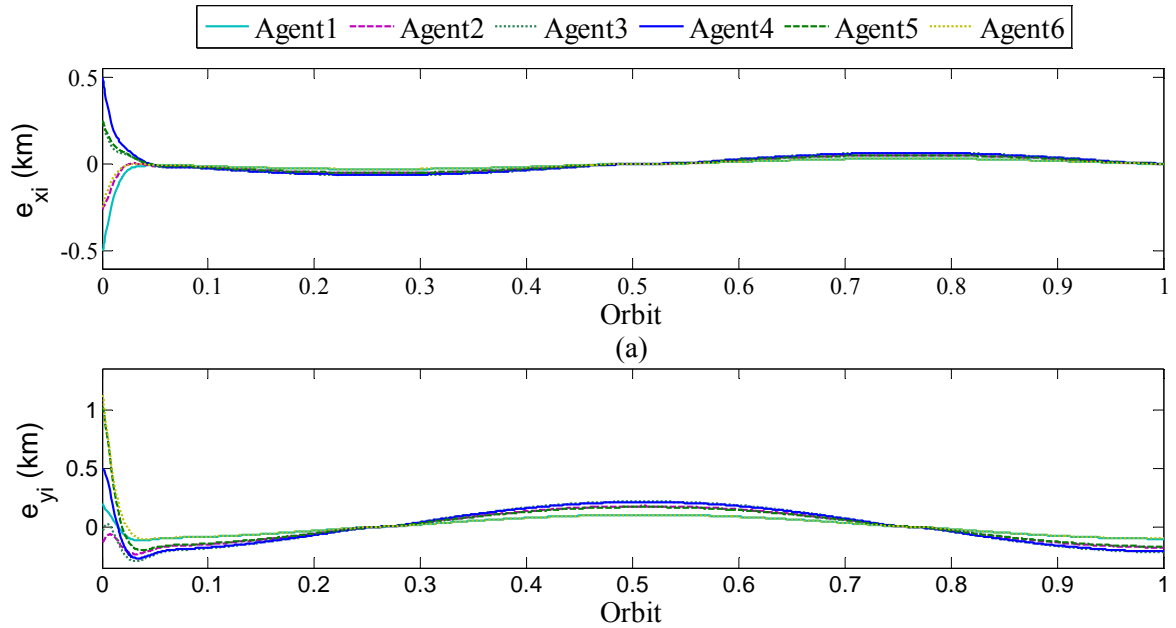

(b)

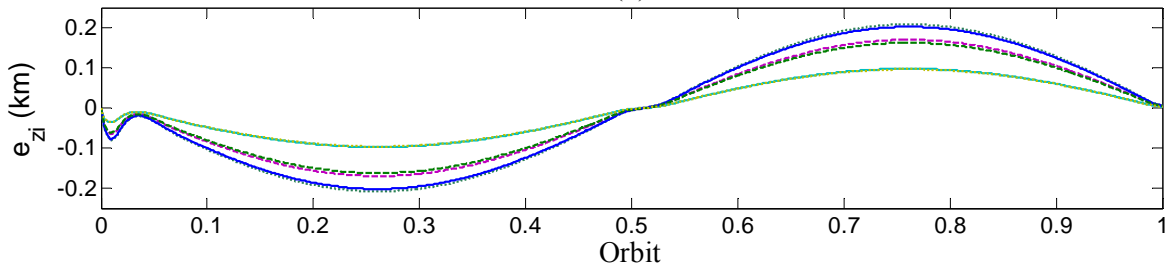

(c)

Figure 3.34: Tracking errors, spacecraft mass $=5 \mathrm{~kg}$

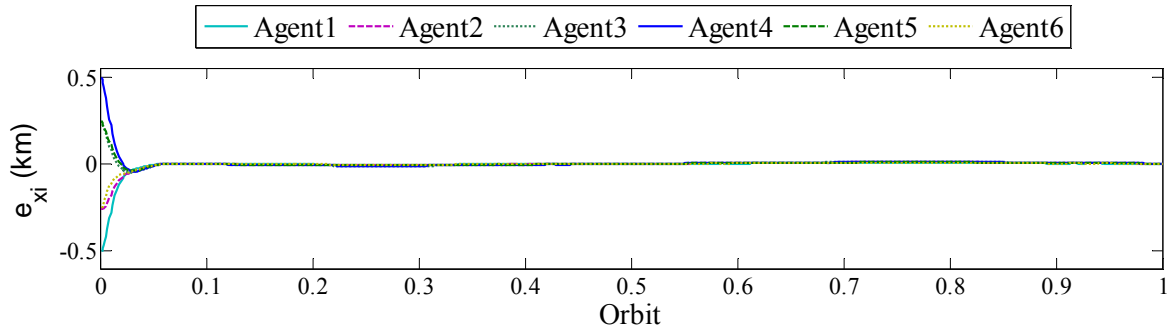

(a)

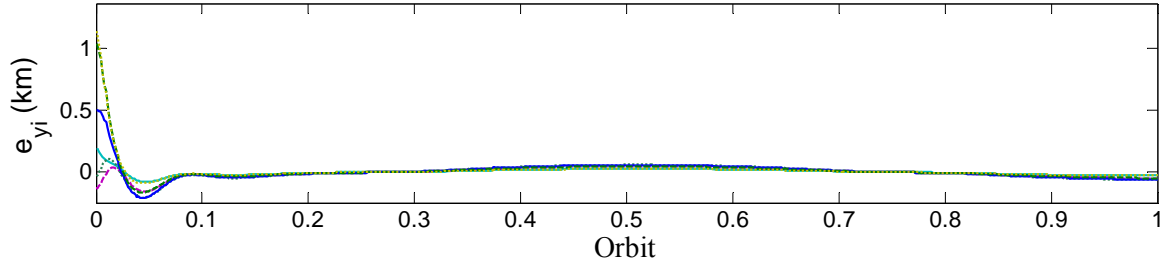

(b)

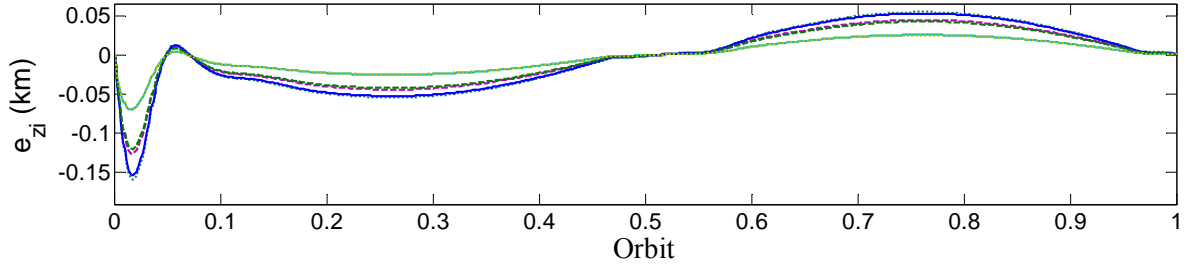

(c)

Figure 3.35: Tracking errors, spacecraft mass $=2 \mathbf{k g}$ 
A nano spacecraft is usually $1 \mathrm{~kg}$ to a maximum $10 \mathrm{~kg}$. Most of the mass is carried at the beginning of the mission and could be propellant mass. During the mission, mass will decrease gradually because of the fuel consumption. It is very practical to model a scenario where mass varies during a mission.

Without changes in the control parameters, Figure 3.33 to Figure 3.35 show the tracking errors of the system when the mass of the spacecraft is at $10 \mathrm{Kg}, 5 \mathrm{Kg}$, and $2 \mathrm{Kg}$. Unfortunately, the control law is not able to reduce the system state error to zero or close to zero. This is because the system equation of motion used for developing the proposed control law has assumed the mass of the spacecraft is $1 \mathrm{Kg}$.

\subsection{CONCLUSIONS}

The contribution in this chapter is employing nonlinear SMC to a multi-agent system for a novel mission, orbiting around a slowly rotating asteroid. Furthermore, consensus control law is applied to investigate the decentralized formation flying system. The system dynamics are simulated with the proposed system requirements. The control law developed is capable of fulfilling these requirements with the consideration of a relatively large initial position and velocity state errors, and sensors faults. However, the results obtained from changing the spacecraft mass show that the proposed control law is not capable of reducing the tracking error to zero. In addition, the results obtained from simulating sensor faults have also suggested receiving a constant or a zero value from a sensor is less vital than having the signal in the reversed sign. A possible decision-making time interval for this scenario can be obtained if a similar fault happens during the mission. Finally, when $5 \mathrm{mN}$ of thrust saturation is included in the simulation with the large initial state error, all 6 agents are not able to track the desired trajectory.

In the next chapter, a robust control law will be developed as an add-on to the current control law. The unsuccessful scenarios in this chapter will be investigated again in the next chapter. 


\section{ROBUST CONTROL OF CONSENSUS SPACECRAFT FORMATION FLYING}

Robust control should guarantee closed-loop stability and acceptable performance not only for the nominal plant model but also for a family of plants. The family is defined to be just large enough to contain all of the possible expected dynamic plant behaviours. The sliding mode control developed in Chapter 3 is applied to the proposed robust control law to stabilize the nominal plant model, shown in Chapter 2.1.3. Whereas, a new technique is incorporated to deal with other possible expected dynamic plant behaviours. Therefore, the control law is robust against a bounded disturbance signals, noise interferences, unmodelled plant dynamics and plant parameter variations. Furthermore, a detailed proof of stability by the Lyapunov theorem for the closed-loop system is formulated. Moreover, the results of numerical simulations incorporating different fault scenarios are presented for a detailed assessment of the system performance under the proposed control strategies and validation of the established theoretical framework. Finally, some brief conclusions are presented.

\subsection{Design of Control LaWs}

An uncontrolled spacecraft can deviate from its desired orbit due to external disturbances. Moreover, the initial state of the spacecraft will be different from its desired state which leads to an initial state error. In the sliding mode technique, these factors can increase the magnitude of the reaching phase from the control system's perspective. If the magnitude of the reaching phase grows, the system may become unstable.

The control law is designed based on a generalized framework that is provided in Chapter 3.1.2. To simplify the control design procedure, Equations (2.37) to (2.39) are re-formulated in the following state-dependent parameterized form. 


$$
\left[\begin{array}{c}
\dot{x} \\
\dot{y} \\
\dot{z} \\
\ddot{x} \\
\ddot{y} \\
\ddot{z}
\end{array}\right]=\left[\begin{array}{cccccc}
0 & 0 & 0 & 1 & 0 & 0 \\
0 & 0 & 0 & 0 & 1 & 0 \\
0 & 0 & 0 & 0 & 0 & 1 \\
0 & 0 & 0 & 0 & 2 \varpi_{T} & 0 \\
0 & 0 & 0 & -2 \varpi_{T} & 0 & 0 \\
0 & 0 & -\varpi_{T}{ }^{2} & 0 & 0 & 0
\end{array}\right]\left[\begin{array}{c}
x \\
y \\
\dot{x} \\
\dot{y} \\
F_{g x} \\
F_{g y} \\
F_{g z}
\end{array}\right]+\left[\begin{array}{c}
0 \\
0 \\
0 \\
u_{f x}+F_{d x} \\
u_{f y}+F_{d y} \\
u_{f z}+F_{d z}
\end{array}\right]
$$

\subsubsection{Sliding Manifold}

The sliding manifold is defined in Chapter 3.1.3, the compact form is rewritten as follows

$$
s=\alpha_{v}+\mathrm{K}_{1} \alpha_{x}
$$

where $\alpha_{v}$ and $\alpha_{x}$ is defined in Equation (2.65) and Equation (2.66), respectively, and $s=$ $\left(s_{1}{ }^{T}, s_{2}{ }^{T}, \cdots s_{n}{ }^{T}\right)^{T} \in R^{3 n}$.

Lemma 4.1: if the sliding manifold $s=0$ is reached, then the absolute state error $e_{x}$ asymptotically converges to zero.

Proof: Consider the following Lyapunov function

$$
V=\frac{1}{2} \alpha_{x}^{T} \alpha_{x}
$$

If the trajectory of the system is converged to the sliding manifold described by $s=0$, then we have

$$
\alpha_{v}=-\mathrm{K}_{1} \alpha_{x}
$$

The time derivative of the Lyapunov function $V$ defined by Equation (4.3) along with Equations (2.67) and (4.4) result in 


$$
\dot{V}=-\mathrm{K}_{1} \alpha_{x}^{T} \alpha_{x}
$$

which implies that the lumped state error $\alpha_{x}$ asymptotically converges to zero. Relate this to Equation (2.65) and hence the absolute state error $e_{x}$ also converges to zero.

\subsubsection{Control Law Formulation}

The control law for the $i$ th follower agent in the multi-agent system is now given by

$$
u_{i}=g_{i}^{-1}\left(-\mathrm{f}_{i}-\psi_{i}-\mathrm{K}_{2} s_{i}\right), \quad i=1,2, \cdots, n
$$

and the compact form can be expressed as follow

$$
u=g^{-1}\left(-F-\psi-\mathrm{K}_{2} s\right)
$$

where $K_{2}$ is a positive constant, and the robust term $\psi_{i} \in R^{3}$, which is determined in the succeeding equations, is used to counteract the external disturbance.

The robust controller $\psi_{i}(i=1,2, \cdots, n)[55]$ in the control law (4.6) is defined as

$$
\psi_{i j} \equiv \kappa_{i} \tanh \left(\frac{3 k_{u} \kappa_{i} s_{i j}}{\epsilon}\right), \quad k_{u}=0.2785, \quad j=1,2,3
$$

where $\kappa_{i}$ is a positive constant satisfying $\kappa_{i} \geq \vartheta_{M i}+\left\|\ddot{x}_{d}\right\|$ and $\epsilon$ is a positive scalar.

\subsubsection{Stability Analysis}

In the following section, the stability proof shows that the control law defined by Equation (4.7) for the closed-loop system (2.49) guarantees semi-global uniform ultimate boundedness of the relative states of the space vehicles to any desired formation.

Theorem 4.1: For the multiple spacecraft formation flying mathematical model in Equation (2.48) to (2.49), the sliding manifold is chosen as Equation (4.2), the control law is defined as Equation (4.7), and the bounds on the external disturbances on the system is assumed as given by Equation 
(2.34) to (2.36). If initial conditions satisfies $V(0) \leq V_{m}$, where $V_{m}$ is any positive constant, then $s$ is semi-global uniformly ultimately bounded.

Proof: Consider the following Lyapunov function,

$$
V=\frac{1}{2} s^{T} M^{-1} s
$$

by taking the time derivative, we can have

$$
\dot{V}=s^{T} M^{-1} \dot{S}
$$

Multiplying $M^{-1}$ to the time derivative of the sliding manifold from Equation (4.2), and substitute Equation (2.68) to replace $M^{-1} \dot{\alpha}_{v}$ yields

$$
M^{-1} \dot{S}=\mathrm{F}+g \mathrm{u}+\vartheta-X_{d}+\mathrm{K}_{1} M^{-1} \alpha_{v}
$$

Substituting the control law (4.7) to Equation (4.11) yields

$$
M^{-1} \dot{s}=-\psi-\mathrm{K}_{2} s+\vartheta-X_{d}+\mathrm{K}_{1} M^{-1} \alpha_{v}
$$

where $K_{2}$ is a positive constant and $\psi=\left(\psi_{1}{ }^{T}, \psi_{2}{ }^{T}, \cdots \psi_{n}{ }^{T}\right)^{T}$.

The following inequality with respect to the robust controller $\psi_{i}(i=1,2, \cdots, n)$ can be obtained from [55], as shown below.

$$
s_{i}{ }^{T}\left(\vartheta_{i}-\ddot{x}_{d}\right)-s_{i}{ }^{T} \psi_{i} \leq \sum_{j=1}^{3}\left|s_{i j}\right|\left(\vartheta_{M i}+\left\|\ddot{x}_{d}\right\|\right)-\sum_{j=1}^{3} s_{i j} \psi_{i j} \leq \epsilon
$$

By applying the above inequality we have 


$$
s^{T}\left(\vartheta-X_{d}\right)-s^{T} \psi \leq \sum_{i=1}^{n}\left(s_{i}{ }^{T}\left(\vartheta_{i}-\ddot{x}_{d}\right)-s_{i}^{T} \psi_{i}\right) \leq n \epsilon
$$

and by using the well-known inequality

$$
\left(\sqrt{c} S-\frac{\mathrm{K}_{1} M^{-1} \alpha_{v}}{2 \sqrt{c}}\right)^{T}\left(\sqrt{c} S-\frac{\mathrm{K}_{1} M^{-1} \alpha_{v}}{2 \sqrt{c}}\right) \geq 0
$$

Equation (4.16) can be obtained

$$
\mathrm{K}_{1} s^{T} M^{-1} \alpha_{v} \leq c s^{T} s+\frac{\mathrm{K}_{1}{ }^{2} \sigma^{2}}{4 c}
$$

where $c$ is a positive constant satisfying $c<\mathrm{K}_{2}$. According to Lemma 3.1, if $V \leq V_{m}$, then $\|s\|$ is bounded; furthermore, $\left\|M^{-1} \alpha_{v}\right\|$ is bounded, therefore $\sigma \geq\left\|M^{-1} \alpha_{v}\right\|$.

Substituting Equation (4.12) to Equation (4.10) yields

$$
\dot{V}=s^{T} M^{-1} \dot{s}=-s^{T} \psi-\mathrm{K}_{2} s^{T} s+s^{T}\left(\vartheta-X_{d}\right)+\mathrm{K}_{1} s^{T} M^{-1} \alpha_{v}
$$

By applying the inequalities of (4.14) and (4.16) to Equation (4.17), $\dot{V}$ can be expressed as follows

$$
\dot{V} \leq-\left(\mathrm{K}_{2}-c\right) s^{T} s+\frac{\mathrm{K}_{1}^{2} \sigma^{2}}{4 c}+n \epsilon
$$

where $\mathrm{K}_{1}$ and $\mathrm{K}_{2}$ are design parameters. Thus, $\dot{V} \leq 0$ when $s$ is outside of the set

$$
\left\{s:\|s\| \leq \sqrt{\frac{\frac{\mathrm{K}_{1}^{2} \sigma^{2}}{4 c}+n \epsilon}{\mathrm{K}_{2}-c}}\right\}
$$


which $\mathrm{K}_{1}$ will be a small positive number and $\mathrm{K}_{2}$ will be a sufficiently large positive number. This set implies that $\|s\|$ decreases when $s$ is outside the compact set; therefore, it is concluded that $s$ is semi-global uniformly ultimately bounded. The boundedness of $s$ implies that $\alpha_{x}$ and $\alpha_{v}$ are also bounded.

\subsection{RESULTS AND DisCUSSIONS}

To study the effectiveness and performance of the proposed consensus control strategies, a comprehensive response is simulated using the set of governing equations of motion, Equation (2.49), with the dynamic model of the perturbation of slowly rotating and irregularly shaped body, Equation (2.22). The formation reference trajectory is in an elliptical orbit with perigee altitude of $3 \mathrm{~km}$. Results are obtained by applying. In the following chapter, besides asteroid Castalia, three other asteroids is also examined. They are asteroid Gaspra, Ida and Vesta. The formation reference trajectory is in an elliptical orbit with perigee altitude of $3 \mathrm{~km}$ for asteroid Castalia, $25.5 \mathrm{~km}$ for Gaspra, $43.4 \mathrm{~km}$ for Ida, and $640 \mathrm{~km}$ for Vesta. Simulation results are obtained by applying the proposed control law (4.7) with the following parameters, $m_{s}=1 \mathrm{~kg}, \Omega=i=0^{\circ}, e=0$, for all asteroids. The values for the geometric properties used for simulation for each asteroid are listed in Table 4.1: Asteroid Properties. The basic nonlinear control law developed in Chapter 3 has guaranteed the performance of the spacecraft orbiting around Castalia. The next benchmark is to examine the control law with different asteroids. Each asteroid is chosen because of the discrepancy in its rotation speed, geometry, mass or other parameters. For example, Ida has a similar body rotational speed but higher mass than Castalia, Gaspra has similar mas than Ida, but slower rotational speed, and Vesta has the most outstanding mass out of all.

Table 4.1: Asteroid Properties

\begin{tabular}{|l|c|c|c|c|}
\hline Asteroid & $2 \pi / \omega$ (hour) & $\mu\left(\mathrm{km}^{3} / \mathrm{s}^{2}\right)$ & $C_{20}$ & $C_{22}$ \\
\hline Castalia & 4.07 & $9.4 \times 10^{-8}$ & $-7.275 \times 10^{-2}$ & $2.984 \times 10^{-2}$ \\
\hline Gaspra & 7 & $3.0674 \times 10^{-4}$ & $-7.29 \times 10^{-2}$ & $3.01 \times 10^{-2}$ \\
\hline Ida & 4.63 & $3.5 \times 10^{-3}$ & $-9.02 \times 10^{-2}$ & $4.08 \times 10^{-2}$ \\
\hline Vesta & 5.3 & 14.2607 & $-5.12 \times 10^{-2}$ & $0.55 \times 10^{-2}$ \\
\hline
\end{tabular}

There are 6 spacecraft in this consensus algorithm simulation, and the net disturbance force acting on each agent is different, as the model defined in Equation (3.41). The same consensus communion link, Equation (3.42), is applied, in order to make the comparison to the results from 
Chapter 3. The control gains used in all the simulations for the sliding manifold $K_{1}$ and control law $K$ are by trial and error, and the values are 0.05 and $1 \times 10^{-5}$, respectively. The robust control parameters are $\epsilon=0.01$ and $\kappa_{i}=0.05$. With the additional control effort from the robust control, it is expected that it will be able to handle more difficult situations, the settling time might be shorter. However, the system control input will be increase in order to compensate for the extra system integrity.

\subsubsection{Mission Scenarios}

In this chapter, the same consensus formation configuration is used as defined in Chapter 3.2. Each agent is $0.5 \mathrm{~km}$ away from the center of the formation, and forms a regular hexagon as shown in Figure 3.2. The centre of the formation lies on the desired trajectory. The same sets of initial states are used in this chapter as defined in Chapter 3.2.1. Referencing to the previous results, it is expected that the velocity errors are more vital than the initial position errors. All 6 agents are started 0.1 unit apart from each other in a line along the $\mathrm{x}$ axis. As stated, the initial state error is specially picked and more focus is on the velocity errors. There are three features in the velocity state error, the velocity error in the x-direction stays the same but changes between positive and negative; the velocity error in the y-direction fluctuates between -0.6 to 0.7 ; and the velocity error in the z-direction remains constant for all agents.

For the lesser error case (set 2), this initial state only applies to asteroid Castalia, there is different arrangement for asteroid Gaspra, Ida, and Vesta. Due to the change in geometry and asteroid, the response from the control law is expected to be different. The reference trajectory remains the same in an elliptical shape but with different radius. The orbital period for the desired trajectory is the same, see Chapter 4 for reference. However, the initial state error is set up differently in the advantage of the control law, to compensate for not changing the orbit period of the desired trajectory. The radius used for Gaspra, Ida and Vesta are $25.5 \mathrm{~km}, 43.4 \mathrm{~km}$, and 640 $\mathrm{km}$, respectively.

System control effort depends highly on the reference trajectory and the altitude of the spacecraft, since the reference trajectory is not tailored to each asteroid, therefore, the system control input is expected to be higher, $5 \mathrm{~N}$ is used. Since the control law has the background operating around asteroid Castalia, the simulation starts with disturbance included in the system. As mentioned in Chapter 2, in order to increase the realism of the numerical simulation, some 
mission requirements are added to the simulation, for example, actuator saturation, system faults, and varying the mass of the agents. Sensors faults have been examined and the result is acceptable, thruster faults are examined in this chapter.

In the following section, there are different scenarios where the control law developed in this chapter is examined. With the numerical simulation performed in Chapter 3 , the basic cases were not shown. In Chapter 4.2.2, the spacecraft formation flying system is examined with the large initial state errors comprising the disturbances model explained in Chapter 2.1.5. The results for the nominal initial state errors are not shown in this section, but it is applied in Chapter 4.2.3 where thruster faults are introduced. The multi-agent system is orbiting 4 different asteroids in Chapter 4.2.4, to study the performance of the propose control law when multiple actuators' fault is applied to the spacecraft. Last, Chapter 4.2.5 shows the robustness of the control law when the spacecraft mass changes with time.

\subsubsection{Effect of Large Initial State Errors with Disturbances}

Previously, the proposed control law in Chapter 3 was able to control all 6 agent when both large initial state errors and disturbances were applied to the system. However, when the $5 \mathrm{mN}$ thruster saturation condition applied, the control law failed to accomplish the mission. Following the predefined mission and system requirements, these scenarios are to be examined again with the supplement of the robust control. The results are shown below, improvements are noticeable, however, the most difficult case still remains not satisfied.

When comparing the performance with the same case, especially, when it is a multiple agent swarm system, it is very difficult to compare the spacecraft tracking errors and formation error by each individual agents. There are two metrics, numerically combing the performance of all agents in the consensus system helped to simplify the analysis.

The total error of the consensus network is expressed as the summation of the tracking errors for each individual agent as an absolute error metric (AEM). It can be calculated by

$$
\mathrm{AEM}=\sqrt{\sum_{i=1}^{n}\left\|e_{x i}\right\|^{2}}
$$


This metric shows the total error of the system, not each individual spacecraft. The value of the error is accumulated base on the number of agents within the consensus formation.

Decentralized formation flying is unique because the formation of the spacecraft becomes a control parameter. The relative error plays an important role when distributing the control effort. Therefore, the relative error metric (REM) is a good indication for how well the relative error between each spacecraft is maintained.

$$
\mathrm{REM}=\sqrt{\sum_{i=1}^{n-1} \sum_{j=i+1}^{n}\left\|r_{x i j}\right\|^{2}}
$$

The control law was trying to compromise between following the trajectory and getting the agents into the specified formation. The increase and decrease in REM indicates that the system is constantly maneuvering in formation while being offset from the reference trajectory, and out of formation when it is trying to reduce the tracking errors. However, the system converges at the end and both the AEM and REM reduce to zero or close to zero.

In the following results, both the SMC developed in Chapter 3 and the new Robust SMC developed in the current Chapter, are shown to compare the tracking and formation errors. Figure 4.1 and Figure 4.2 are the results of AEM and REM, respectively, for the large initial state error with no thruster saturation applied but included a $3 \mathrm{mN}$ time variant disturbance force. Clearly seen in both the AEM and REM situation, the robust SMC control law developed in this chapter has better performance than the former SMC control law. In both case, the AEM and REM, the robust SMC is below the SMC, this means less errors in the system. The AEM reaches a maximum value of $20 \mathrm{~km}$, and REM reach a maximum value of $26 \mathrm{~km}$. This value is the sum of the error of the total number of agents. In this study, there are 6 agents in the consensus formation system. When dividing these maximum values by 6 , the average of the max tracking error is approximately $3 \mathrm{~km}$, and the average of the maximum 


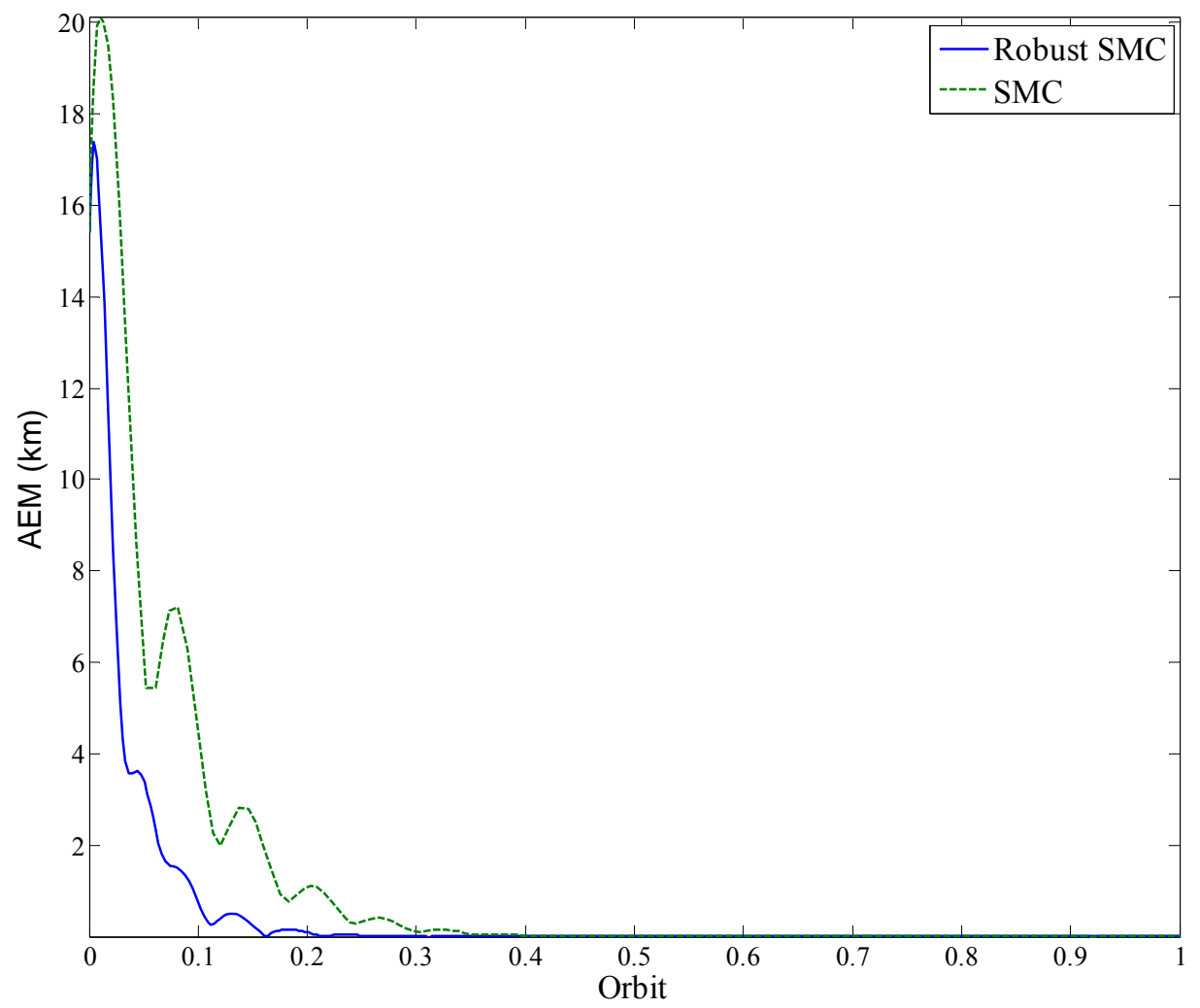

Figure 4.1: AEM (without thruster saturation)

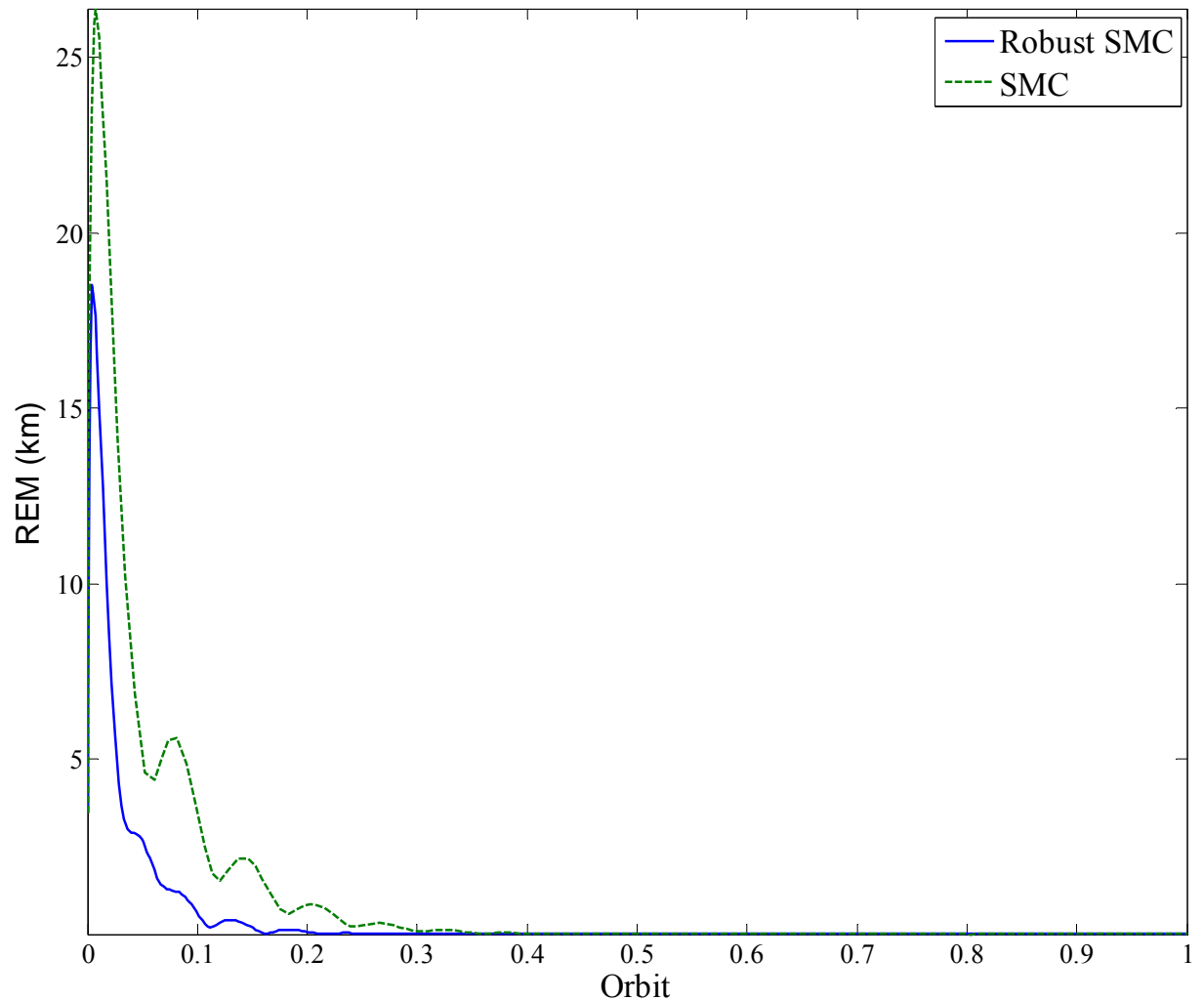

Figure 4.2: REM (without thruster saturation) 
formation error is approximately $4.5 \mathrm{~km}$. The tracking error is caused by the initial position, and the formation error is mainly due to the initial velocity. Similar pattern as the result in Chapter 3, both state errors are dramatically decreased.

Also, a similar response is discovered for the $10 \mathrm{mN}$ thrusters saturation scenario. The errors for the robust SMC have a similar decaying pattern as SMC in both AEM, Figure 4.3, and REM, Figure 4.4. Likewise, as explained in Chapter 3, due to the limitation of the thrusters, state errors propagate a lot larger reflecting the impact of the disturbance from the system.

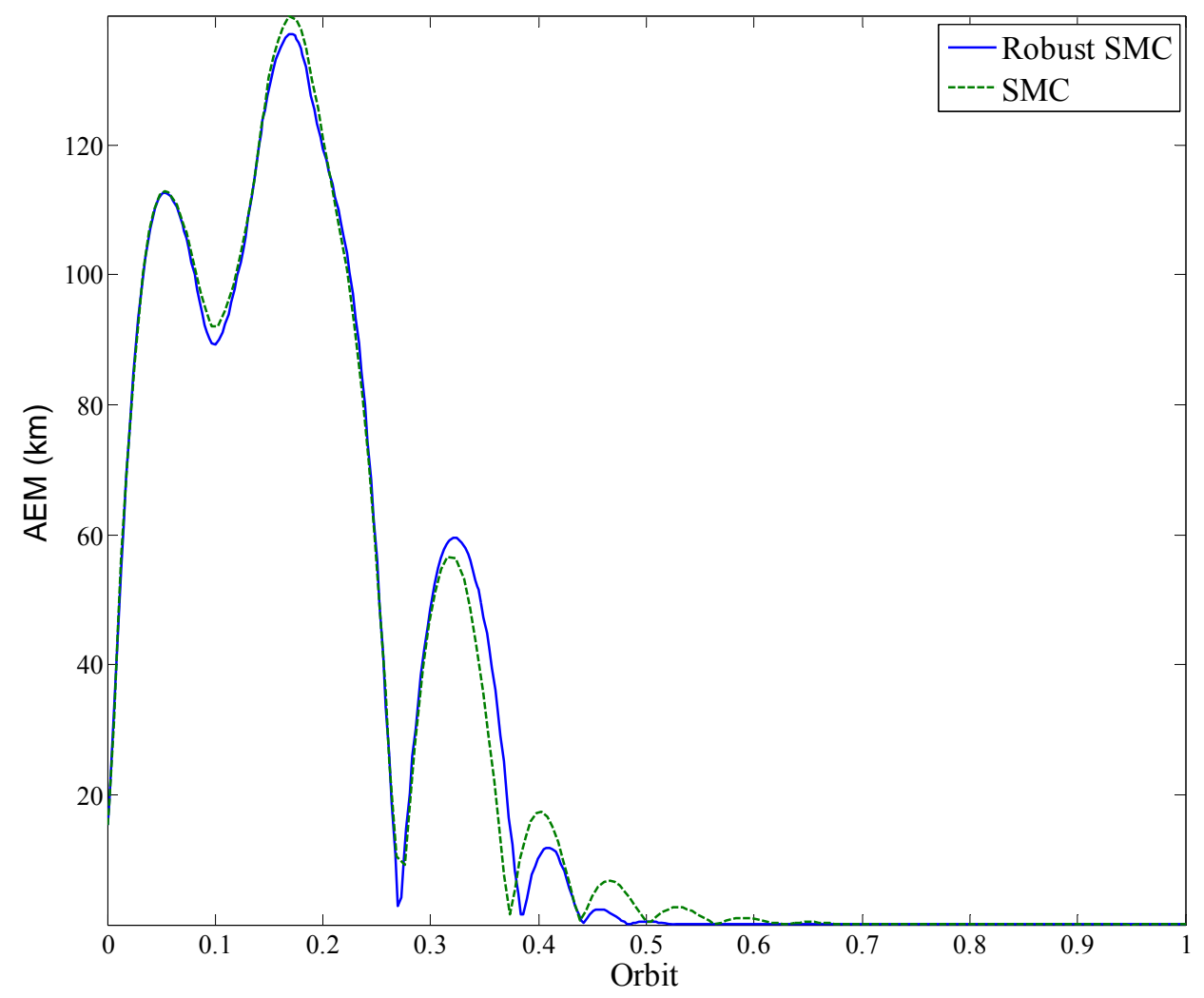

Figure 4.3: AEM (with $10 \mathrm{mN}$ thruster saturation)

Between 0.3 to 0.4 of an orbit in Figure 4.3 and Figure 4.4, the errors in the robust SMC are higher than the SMC. The reason is the robust control is trying to force the consensus system to converge faster. After 0.4 of an orbit, both tracking error in AEM and the formation error in REM have reduced, and the settling time is shorter in robust SMC. 


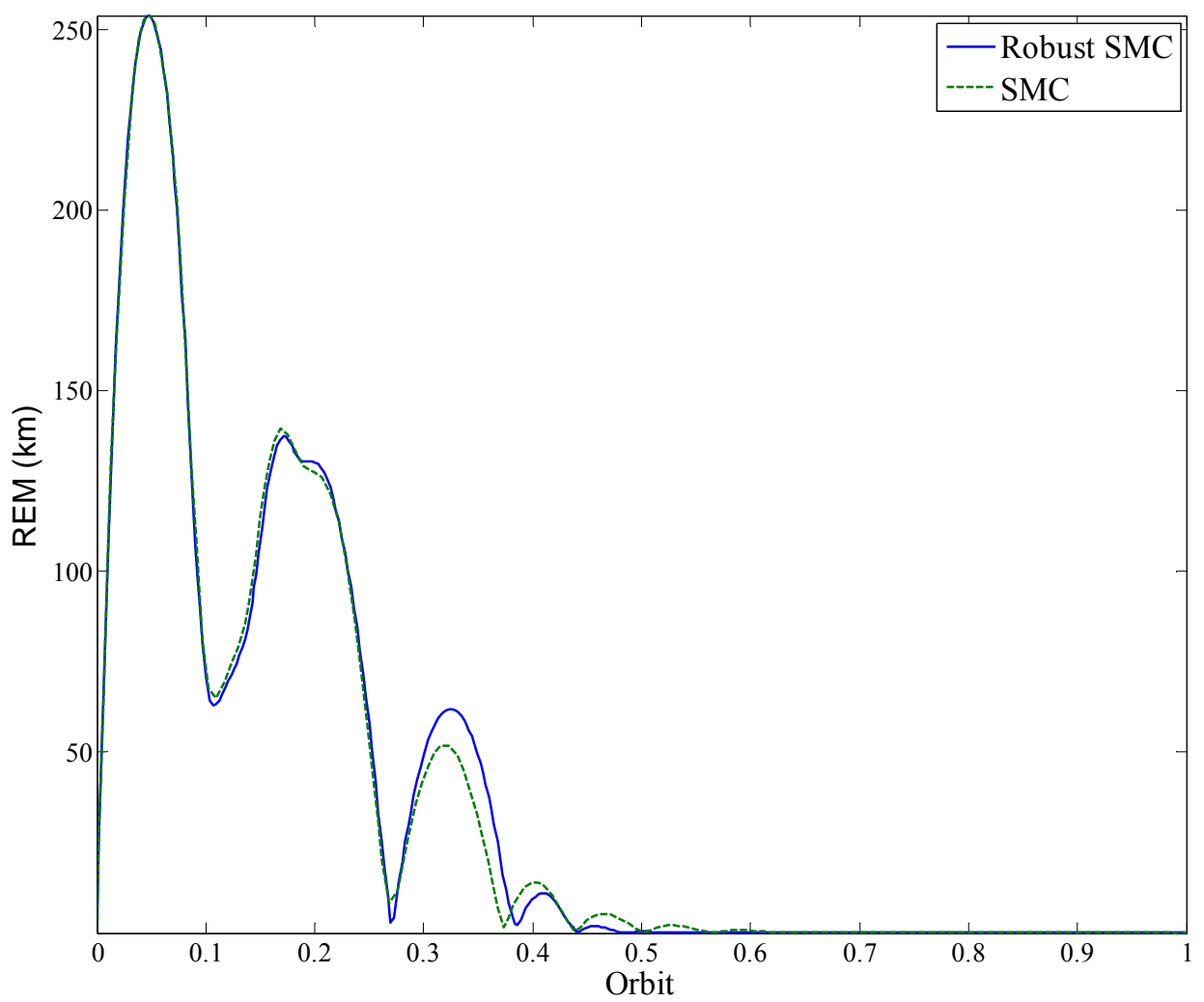

Figure 4.4: REM (with $10 \mathrm{mN}$ thruster saturation)

Figure 4.5 is the control effort required to control the agents in the same scenario with large initial state errors and disturbance while the maximum thrust provide to each agent is $10 \mathrm{mN}$. Coinciding with the AEM and REM graphs, in Figure 4.5, the system is stable roughly after 0.5 of an orbit in all three directions. The control input is mainly responding to the disturbance only. As shown, the steady state required a minimum $3 \mathrm{mN}$ of input force to control the system. If the initial error is included, when no restriction applied, it takes approximately maximum $300 \mathrm{mN}$ and less than 0.03 of an orbit to control the system. Comparing Figure 4.5 with Figure 3.13, the pattern is very similar, however, the total input force required for robust SMC is higher than the SMC. This is the well-known trade-off for robust control. More control effort is required to put the system into steady state, and in a shorter period of time. 


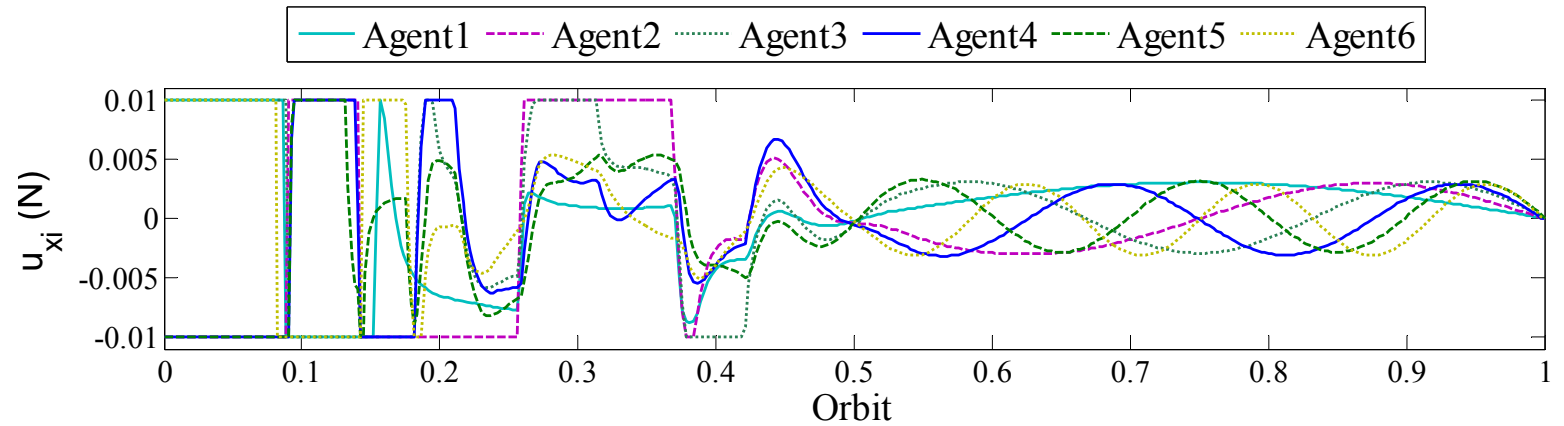

(a)
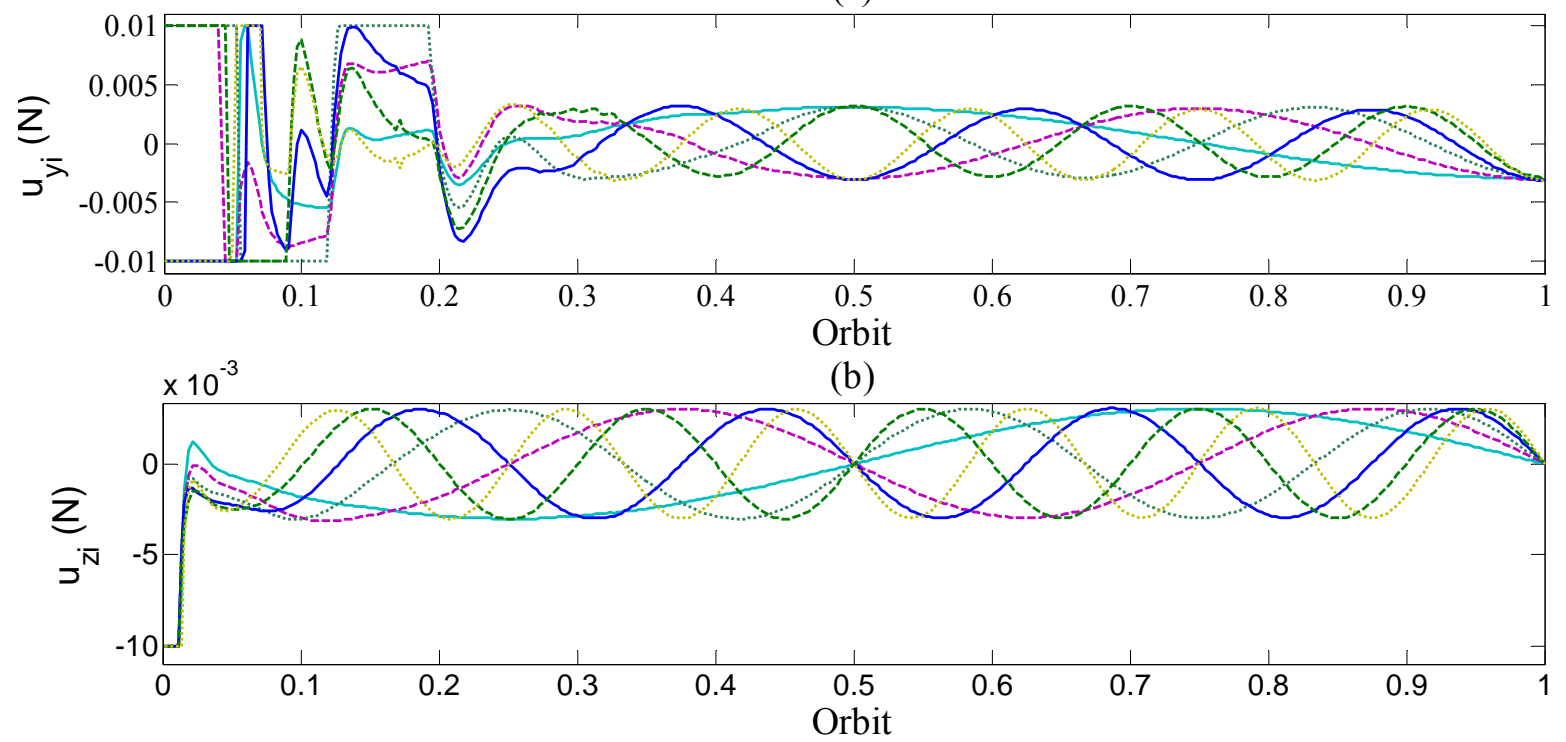

(c)

Figure 4.5: Control input (with $10 \mathrm{mN}$ thruster saturation)

Even though after 3.5 of an orbit in Figure 4.6 and Figure 4.7, the robust SMC is able to reduce the error a lot lower than the SMC. However, a longer period has been examined and the system was not able to reach neither the desired state nor consensus formation. Various control parameters have also been examined, it is reasonable to conclude that this SMC and robust SMC algorithm proposed are not capable to control such large initial error and a large disturbance, relative to the maximum available thrust. The disturbance is equivalent to $60 \%$ of the maximum thrust available, only $40 \%$ of thrust remain to encounter the force from the initial state error. For the $10 \mathrm{mN}$ case, it is noticeable that all 6 agents reach saturation at the beginning, the system was not stable until 0.5 of an orbit. When the thrust reaches its maximum, the agents constantly fly away from the trajectory. It accumulates over time, and the state errors are not abled to recover. This is the same as what happened to the $5 \mathrm{mN}$ saturation scenario. 


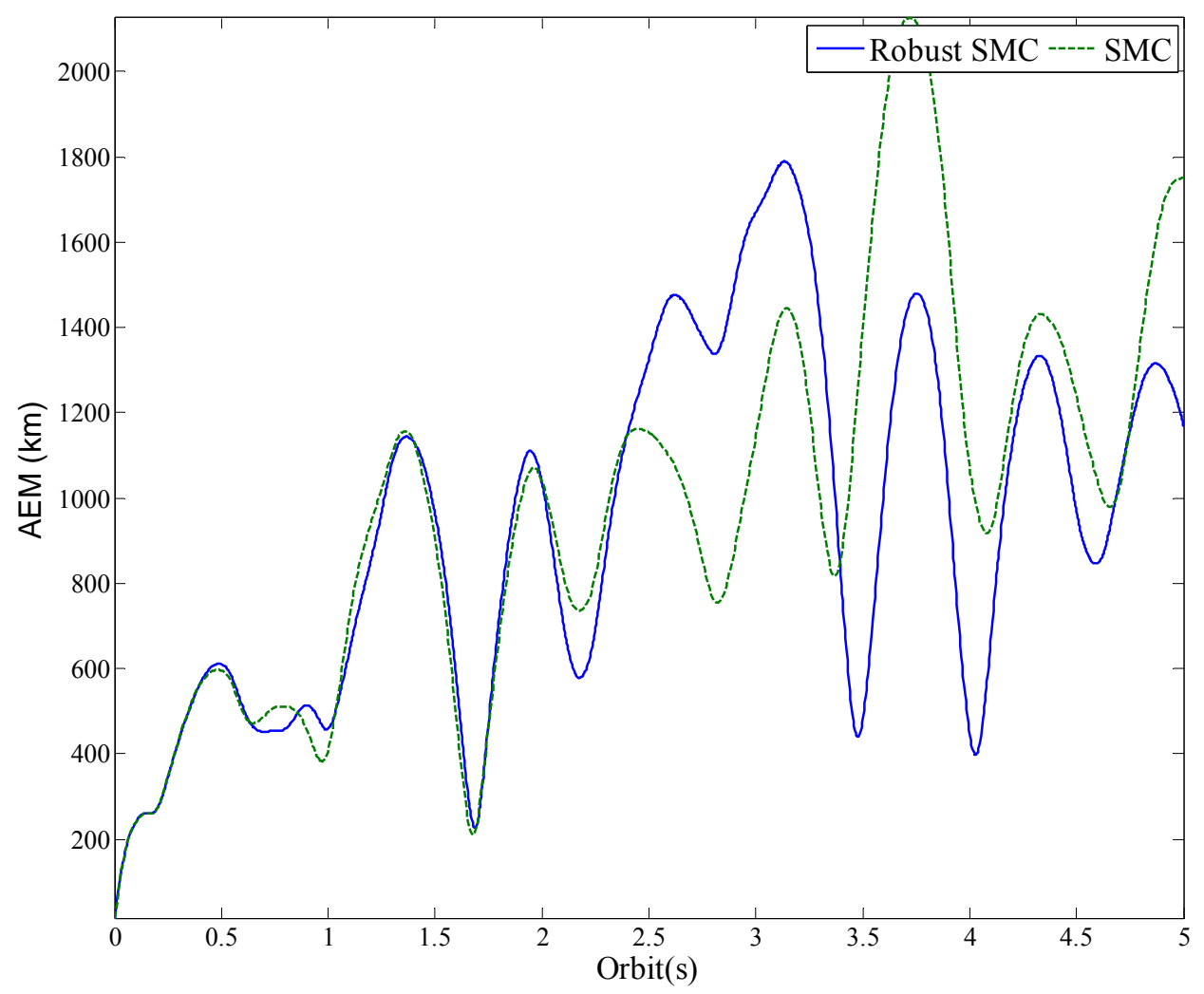

Figure 4.6: AEM (with $5 \mathrm{mN}$ thruster saturation)

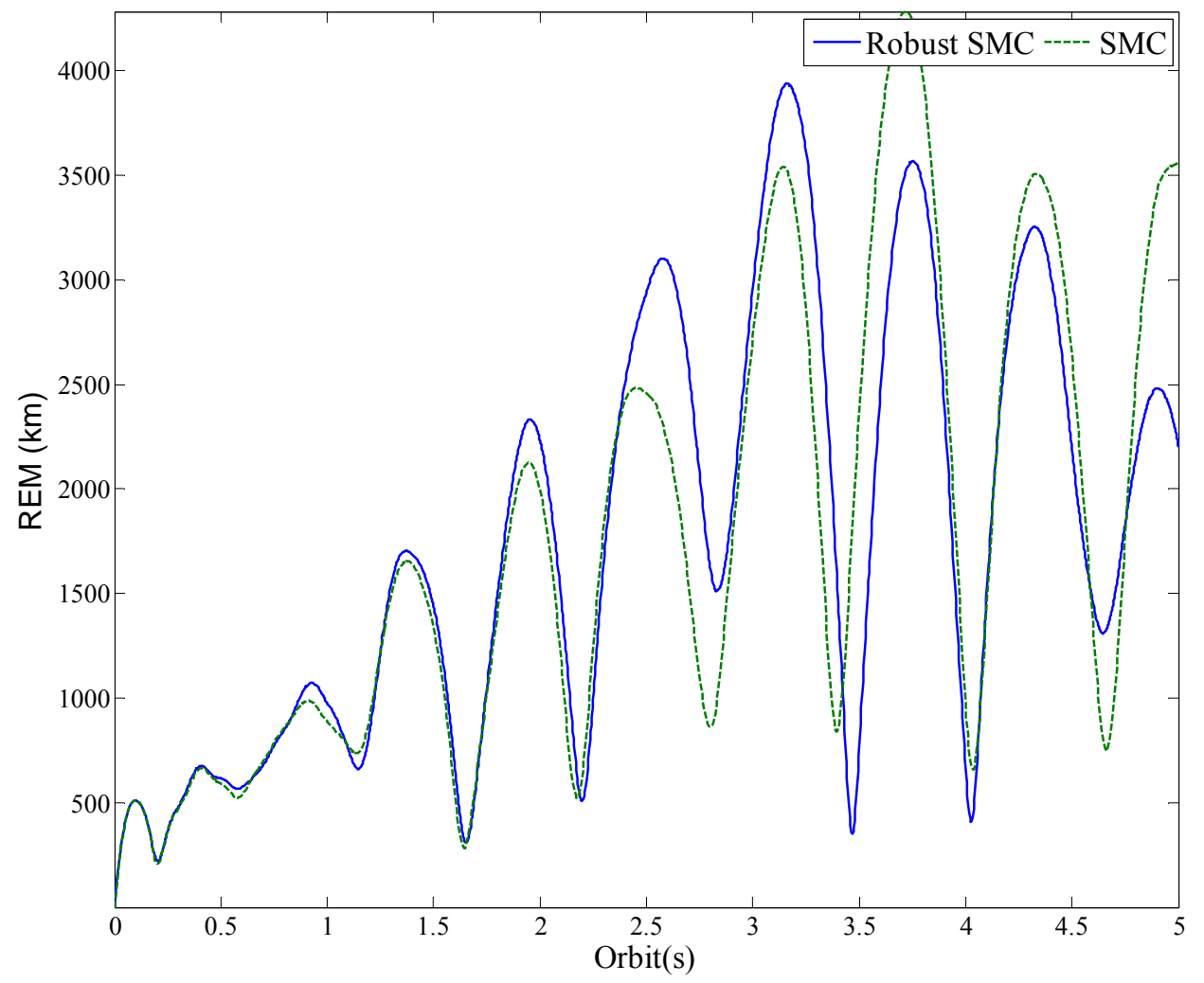

Figure 4.7: REM (with $5 \mathrm{mN}$ thruster saturation) 
In a mission, it is impossible to eliminate initial state error. However, it is not difficult to avoid large initial state errors, if the mission is properly arranged. Especially for the proposed mission, agents are launched from a satellite carrier. The satellite carrier can be placed in a closer distance toward the mission reference trajectory, more importantly; the initial velocity can be avoided which was the main reason that caused the control law to fail in controlling the system. On the other hand, the disturbance model used is very unpleasant to the system when the thruster saturates at $5 \mathrm{mN}$. Although, the proposed scenario is very difficult to control, a modification of the SMC might help in solving the current situation. Terminal sliding mode control is used in the next chapter to examine the same scenario. The developed control law is more than enough to handle this tough situation if $10 \mathrm{mN}$ force is given from the thruster.

\subsubsection{Effect of Thruster Faults}

Saturation is not applied purposely, except for the last set of results, in this section in order to see the full instant response of the control law. Thruster saturation is shown in the scenario when all various thruster faults are applied at the same time in the next section. This simulation is to model when the thrusters in all 3 directions are constantly providing $5 \mathrm{mN}$ of thrust for 5 minutes. As a reminder, 1 reference orbit is approximately 0.5087 hour, therefore, the duration of the downtime is roughly sixteen percent of an orbit. Actuator down time occurs from 400 to 700 seconds, which is 0.2184 to 0.3822 of an orbit. Disturbance is not applied and mild initial state errors are used. With the experience from Chapter 3.2.4, the response has been seen if faults is applied to 3 linked agents, and that is used as a baseline to verify if this reaction is applied to actuator faults. The first case being investigated is only 1 agent, agent 6 , experiencing thruster downtime, which is expected that agent 5 will be affected the most and agent 1 will be the least. The next scenario examines three agents, agents 4,5 , and 6 , experiencing the proposed thrusters fault. Last all six agents experiences the downtime for five minutes.

Reference to Figure 3.22, Figure 4.8 has an identical profile but in a reversed order since this time agent 6 is the source of infection compared with agent 1 being the defected part of the system in Figure 3.22. The actuators error in agent 6 has caused a relatively higher state error in agent 5 than agent 4; agent 4 than agent 3; agent 3 than agent 2; and agent 1 is the least affected because it is the furthest node in the consensus communication network. 

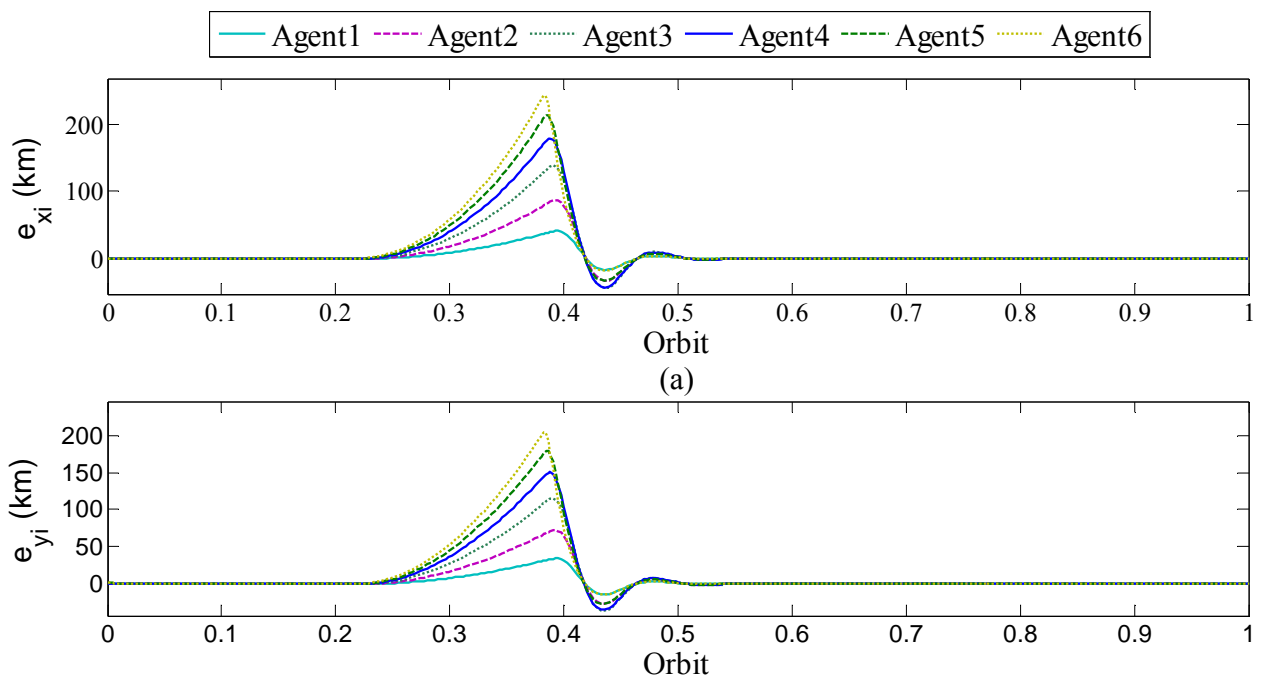

(b)

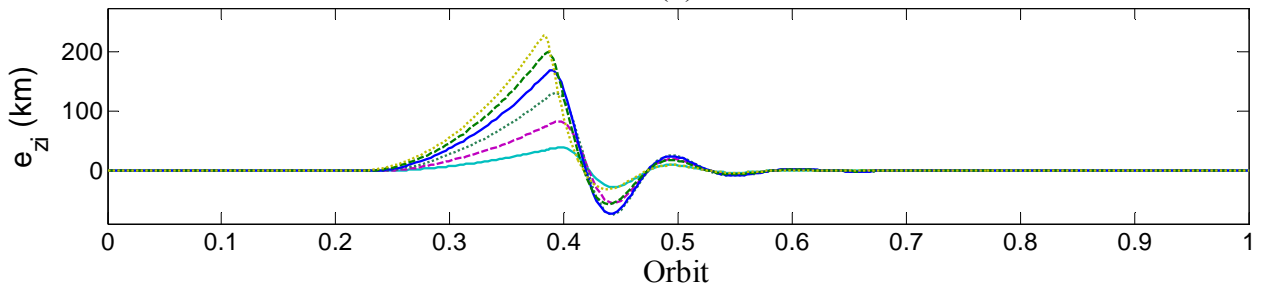

(c)

Figure 4.8: Tracking errors, agent 6’s thruster malfunction

The effect of the thruster fault is more catastrophic than the sensor fault. The maximum state error due to the sensor fault is less than $3 \mathrm{~km}$, whereas the state error due to the thruster faults is more than $200 \mathrm{~km}$. Also, keep in mind that the duration of the system downtime is shorter in this scenario. Though, with such a large state error, with the robust SMC, the settling time of the aftermath is very comparable with the previously developed control law, the error is damped much more effectively and quicker. The downtime ends at 0.3822 of an orbit and the system is settled at 0.689 of an orbit, which took approximately 0.3 of an orbit to settle, this is less than the time it takes for the SMC.

In the system, when there are three agents experiencing actuator faults, Figure 4.9, or all six agents experiencing the same actuator fault but thrust firing in the opposite direction, Figure 4.10, the tracking error for each agent does not increase significantly. Therefore, tracking state errors do not have a strong correlation to the number of agents even in a consensus system. For the specific case as presented in the results of Figure 4.10 ( 6 agents subjected to failure) the settling time is much shorter than the results depicted in Figure 4.9 (3 agents subjected to failure). 


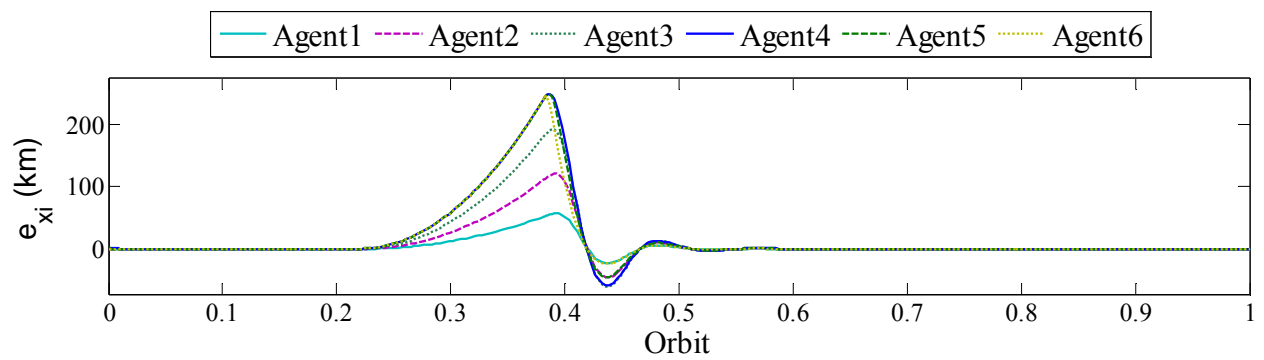

(a)

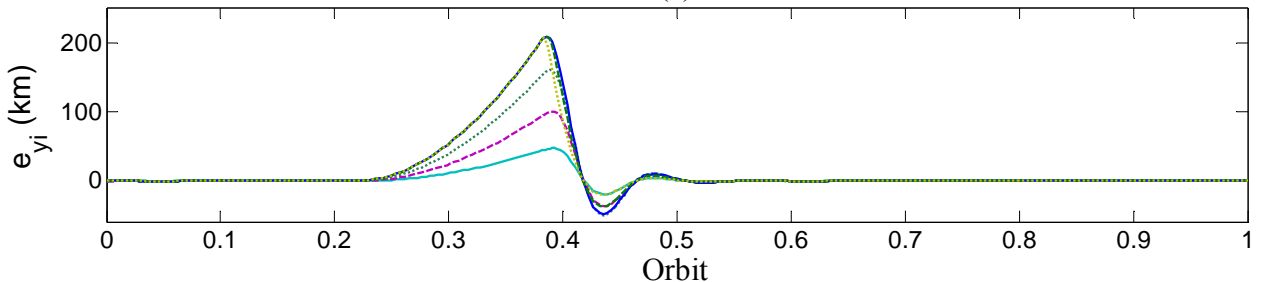

(b)

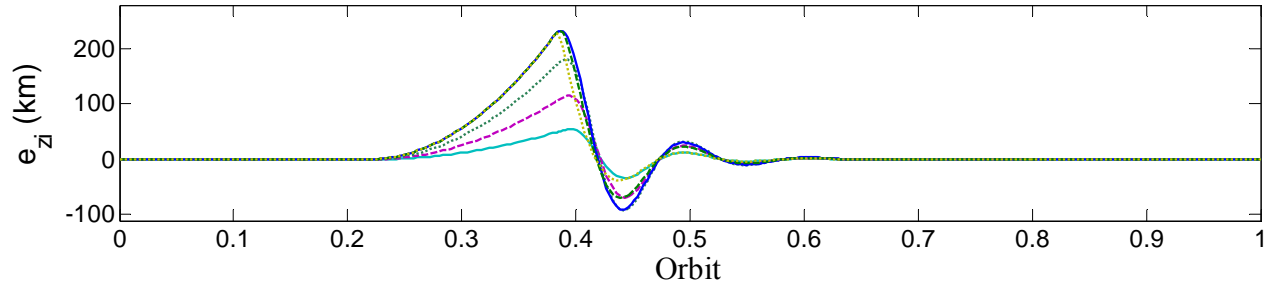

(c)

Figure 4.9: Tracking errors, agent 4, 5, and 6's thruster malfunction

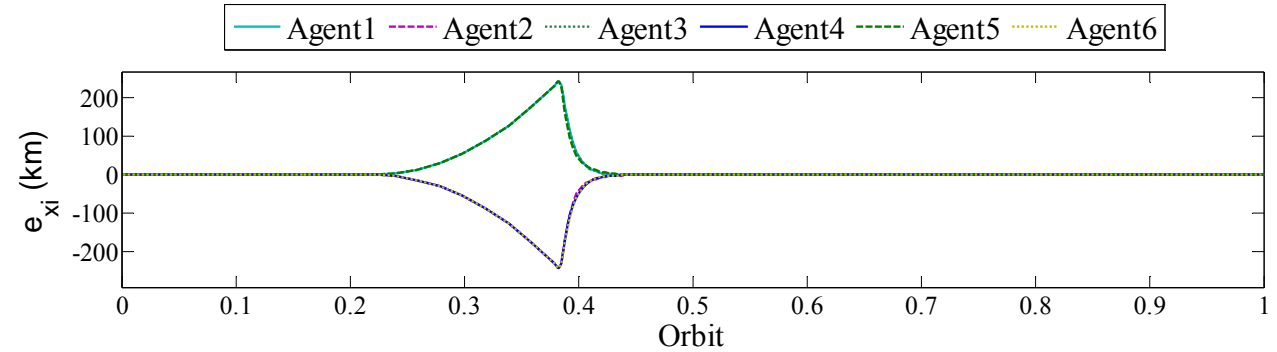

(a)

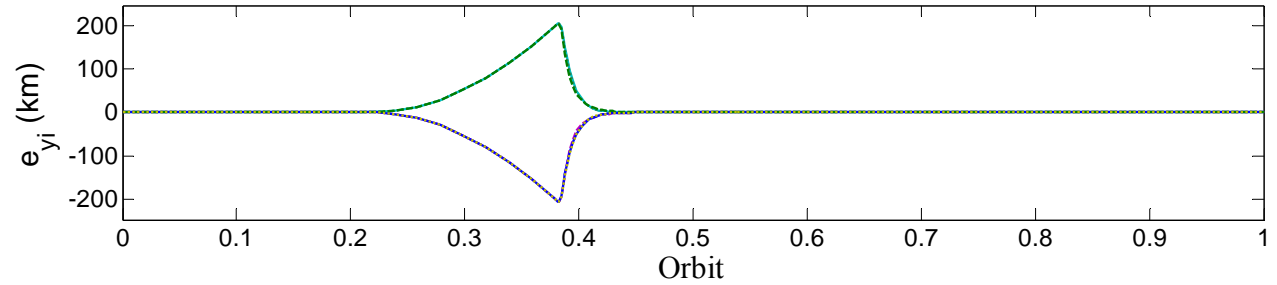

(b)

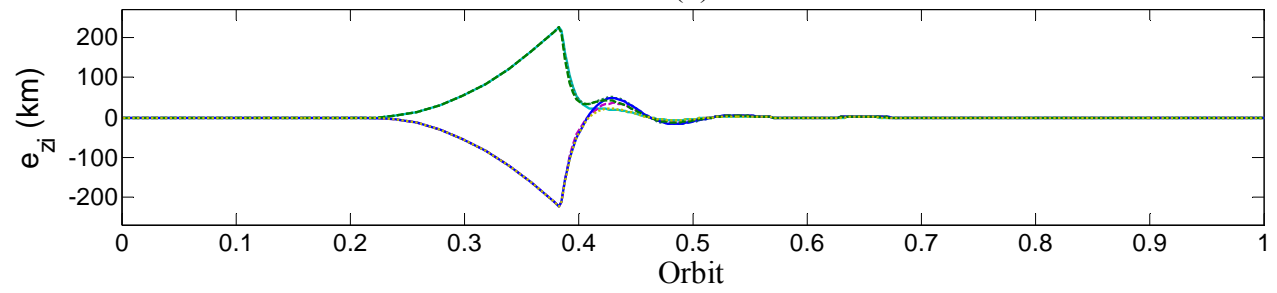

(c)

Figure 4.10: Tracking errors, all 6 agents' thruster malfunction 
When applying the proposed mission restrictions as presented in Chapter 2 to the last scenario, wherein, agents 1, 3, 5 and 2, 4, 6 fire a constant $5 \mathrm{mN}$ and $-5 \mathrm{mN}$ thrust for 5 consecutive minutes, respectively, the robust SMC fails to bring the swarm back to the desired state within 1 orbit, but it is capable of complete the mission in less than 4 orbits, Figure 4.11.
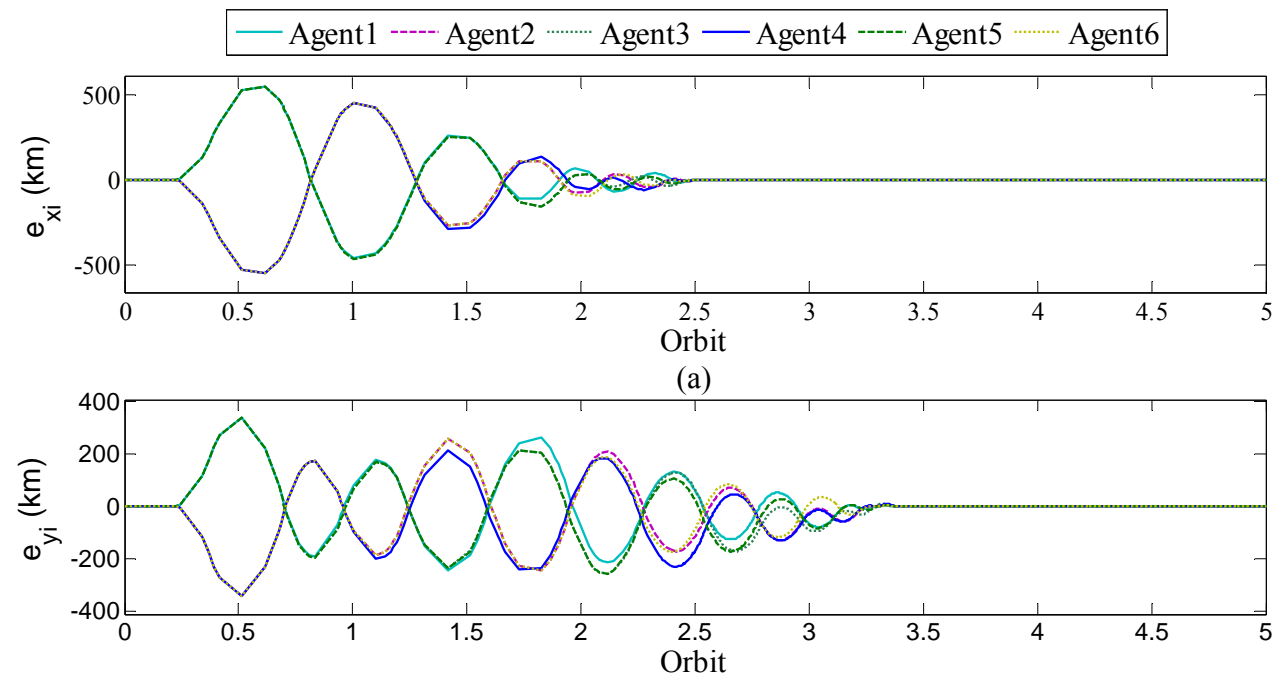

(b)

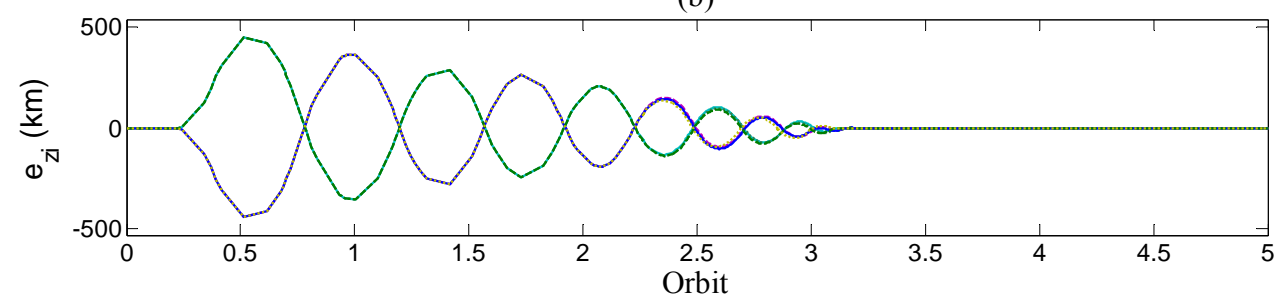

(c)

Figure 4.11: Tracking errors, all 6 agents' thruster malfunction (with $5 \mathrm{mN}$ thruster saturation)

With the $5 \mathrm{mN}$ saturated thrust scenario, see Figure 4.12, maximum thrust is constantly required in order to maintain zero tracking errors, Figure 4.11. The system control input profile for this scenario is very similar to Figure 3.14, which is near the system control limitation. 

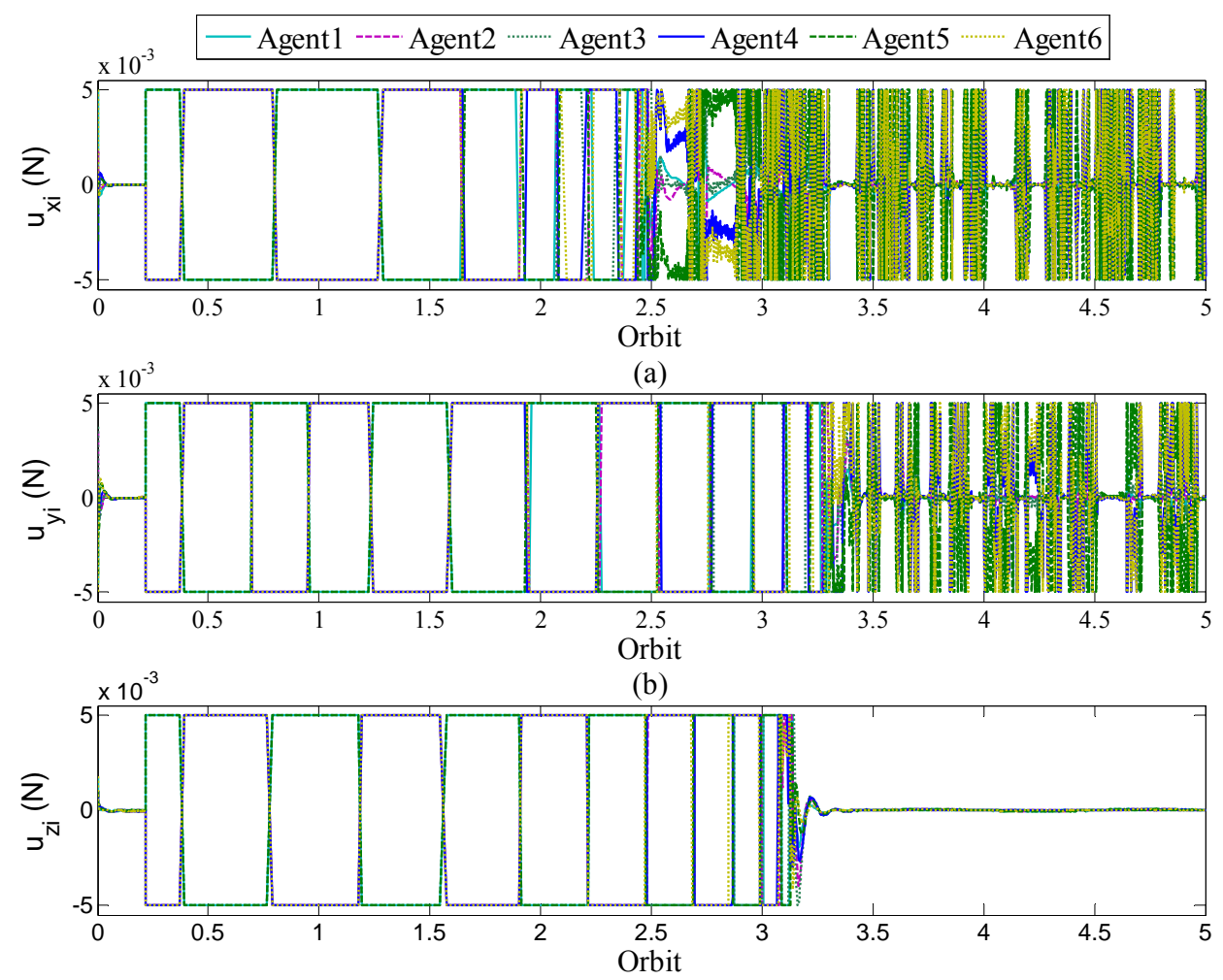

(c)

Figure 4.12: Control input, all six agents' thrusters malfunction (with $5 \mathrm{mN}$ saturation)

The actuator unsaturated cases are shown for studying the behaviour of the system when it is experiencing thruster faults. It is discovered that the way the system responds to actuator faults is very similar to sensors faults; this shows the effect of the consensus system. When one agent from the system is experiencing difficulty to track its reference trajectory, the other agents will follow to maintain the formation. If the effect of the faults continue to grow, and the system is not able to reduce the state error, all agents in the system will fail to track its desired trajectory and maintaining the formation. Therefore, an appropriate trade off function needs to be included in order to separate the defected agent from the system.

\subsubsection{Effect of Multiple Actuator Faults for Different Asteroids}

Numerical simulation is performed for 4 different asteroids in order to show that the control law developed is not design for one specific celestial body only. The 4 asteroids investigated are 4769 Castalia, 951 Gaspra, 243 Ida, and 4 Vesta. The results obtained from the simulations for all 4 asteroids use the same control parameters and reference trajectory model. However, the size of 
the reference trajectory is adjusted corresponding to the dimension of the asteroid, where more details will be given below.

The minimum system control input required for orbiting around asteroid Castalia, Gaspra, and Ida, are similar, and it shows that a nano scale spacecraft is sufficient for exploring these asteroids. In simulation, a maximum of $5 \mathrm{mN}$ thrust per spacecraft is applied for controlling the consensus system. For Asteroid Vesta, due to the geometric properties of this astronomic body, the minimum thrust required to control the spacecraft is $20 \mathrm{mN}$, and for this specific mission, it is assumed that the thruster is saturated at $50 \mathrm{mN}$. In summary, the minimum control input required is different for each asteroid, but the maximum thrust provided for each asteroid is the same, except for Vesta. The robust control parameters for each asteroid are different. $\kappa_{i}=0.050$ for asteroid Castalia; $\kappa_{i}=0.025$ for asteroid Gaspra; $\kappa_{i}=0.035$ for asteroid Ida; $\kappa_{i}=0.030$ for asteroid Vesta. In the following cases, there are 3 different types of actuator faults which are introduced to all 6 agents at the same time. Thrusters are not providing any propulsion for agents 1, 5, and 6 during the downtime, thrusters on agents 2 and 4 provided thrust in the opposite direction, and agent 3 supplies with $2 \mathrm{mN}$ of actuator force in all 3 axial directions. System downtime is the same as the one used previously, it occurs from 400 to 700 seconds, which is 0.2184 to 0.3822 of an orbit.

\subsubsection{4769 Castalia}

Comparing with the results in Chapter 4.2.3, the following results are obtained with the same system dynamics as that aforementioned, however, it is going through a combination of thruster faults. This includes spacecraft being propelled in an opposite direction as to what the control law specifies, thruster not firing, and the actuator providing thrust at a constant value continuously. These situations are reflected in Figure 4.13 at 0.2184 to 0.3822 of an orbit. Afterwards, thrusters reach their saturation point in order to control all 6 agents and bring them back to the desired state at around the $3.75^{\text {th }}$ orbit; the control required after 3.75 of an orbit is the minimum required thrust, see Figure 2.7. This phenomena shows that the system is stable and it agrees with the results shown in Figure 4.14. 

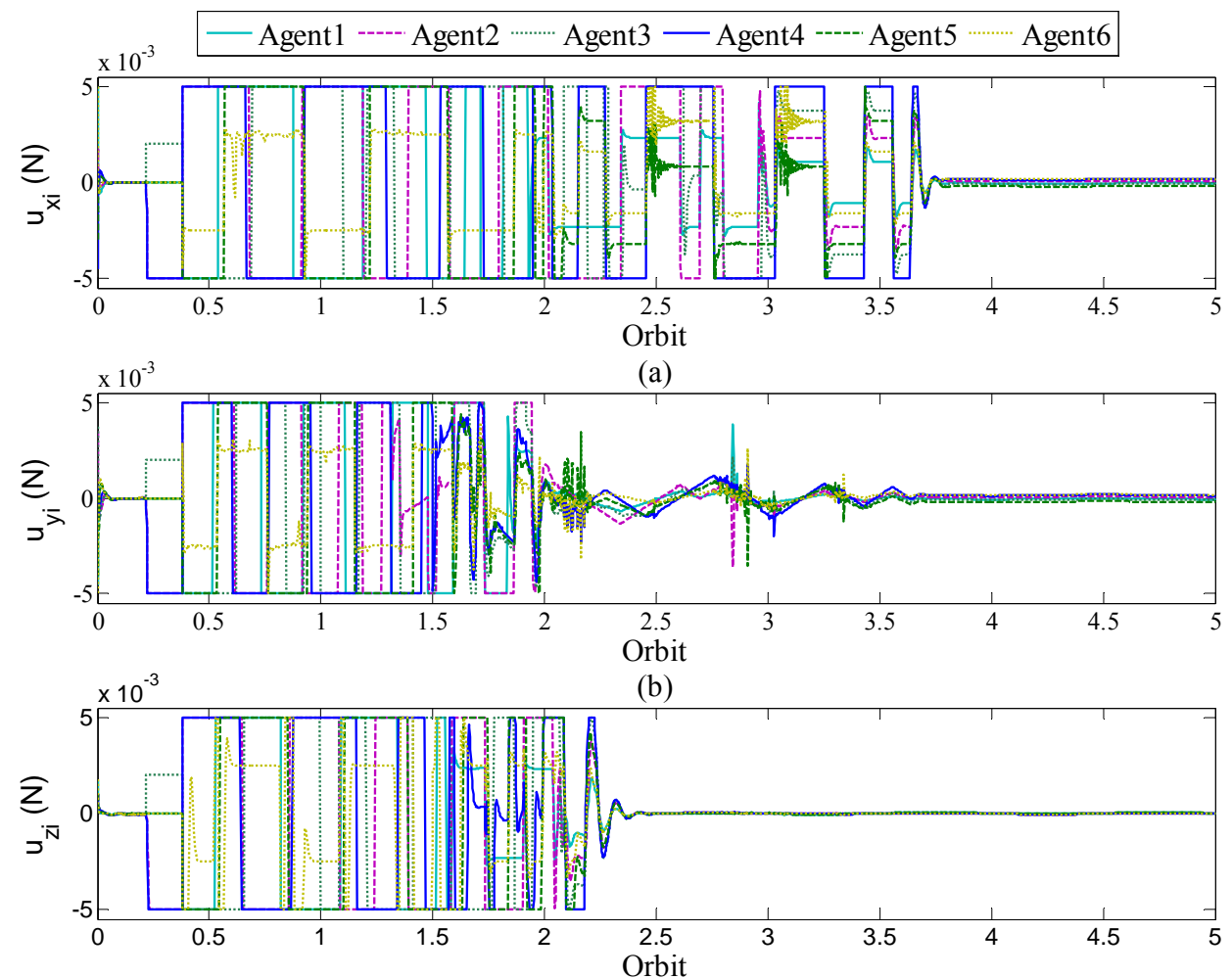

(c)

Figure 4.13: Control input, all agents' thruster malfunction
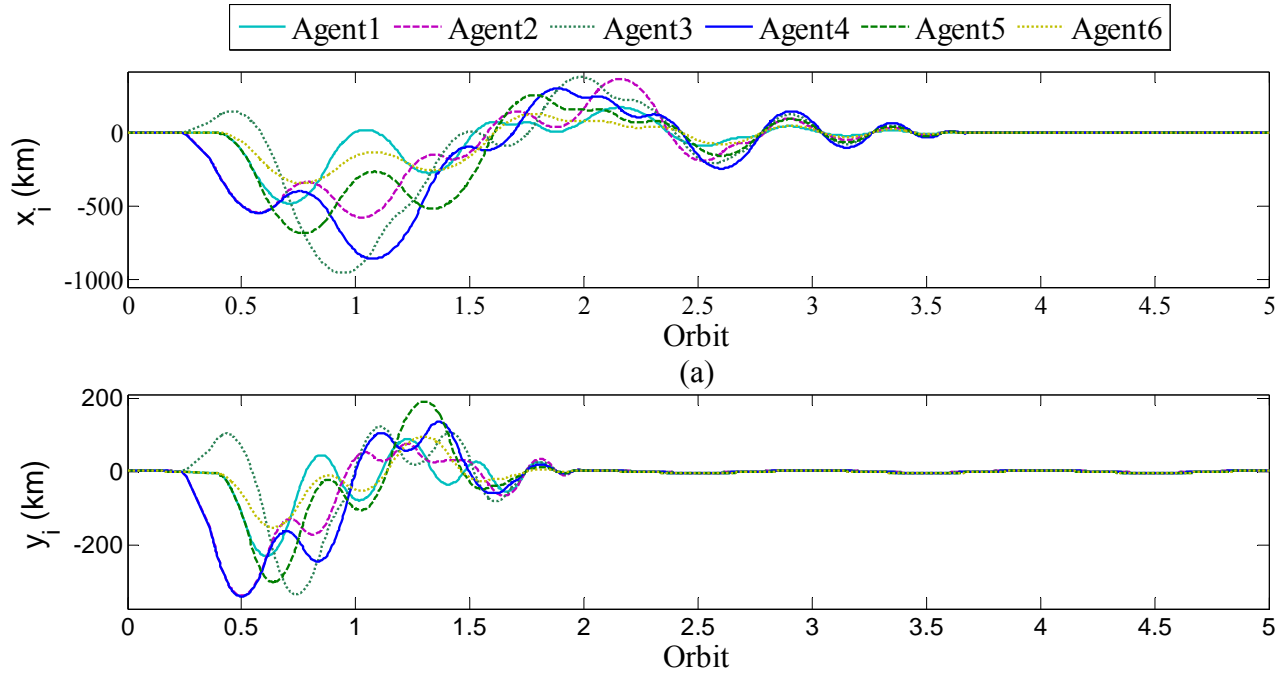

(b)

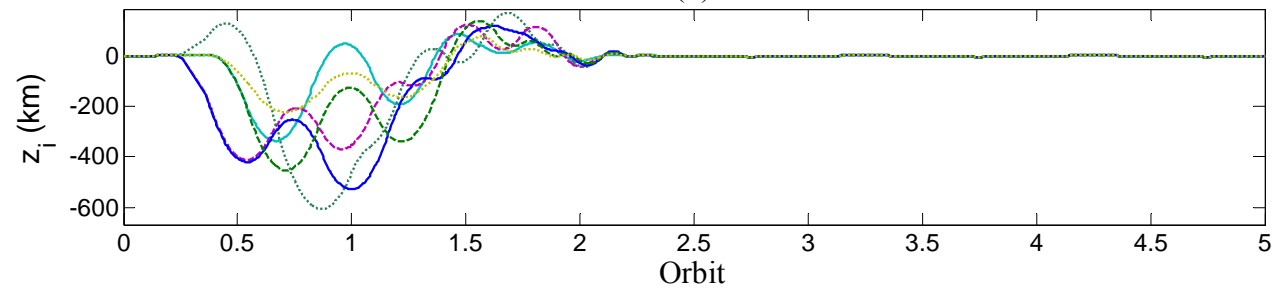

(c)

Figure 4.14: State of all agents', thruster malfunction 
In Figure 4.15, the state error in the $\mathrm{x}$-direction is higher than the $\mathrm{y}$ and $\mathrm{z}$ directions. As far as the author's knowledge, this has no identifiable reason since different conclusions are drawn for different asteroids. However, this can be caused by the duration of the downtime and the time where the faults occur.
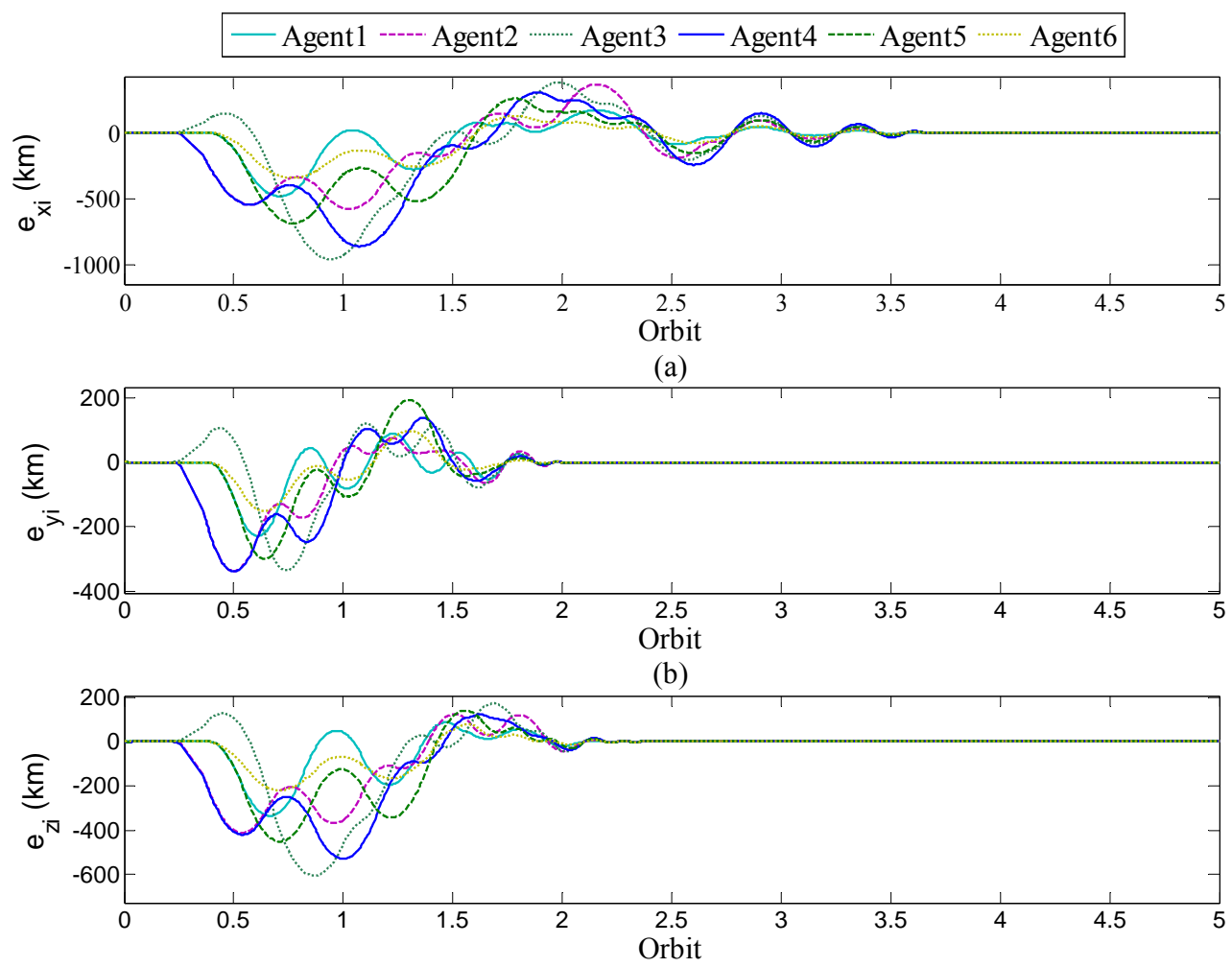

(c)

Figure 4.15: Tracking errors, all agents' thruster malfunction

Based on the results, it is concluded that the proposed control law is capable to control all 6 agents and maintain proper formation while following the desired trajectory even when multiple actuator faults occur during the mission.

\subsubsection{951 Gaspra}

Asteroid Gaspra has very similar dynamic properties to asteroid Castalia. It is because the value of the $2^{\text {nd }}$ degree and $2^{\text {nd }}$ order gravity coefficients, $C_{20}$ and $C_{22}$, are very comparable to asteroid Castalia. However, the dimension and the mass of this asteroid is much greater than that of Castalia. The spin rate is almost twice as slow, and it is the slowest of the 4 asteroids that are investigated. 

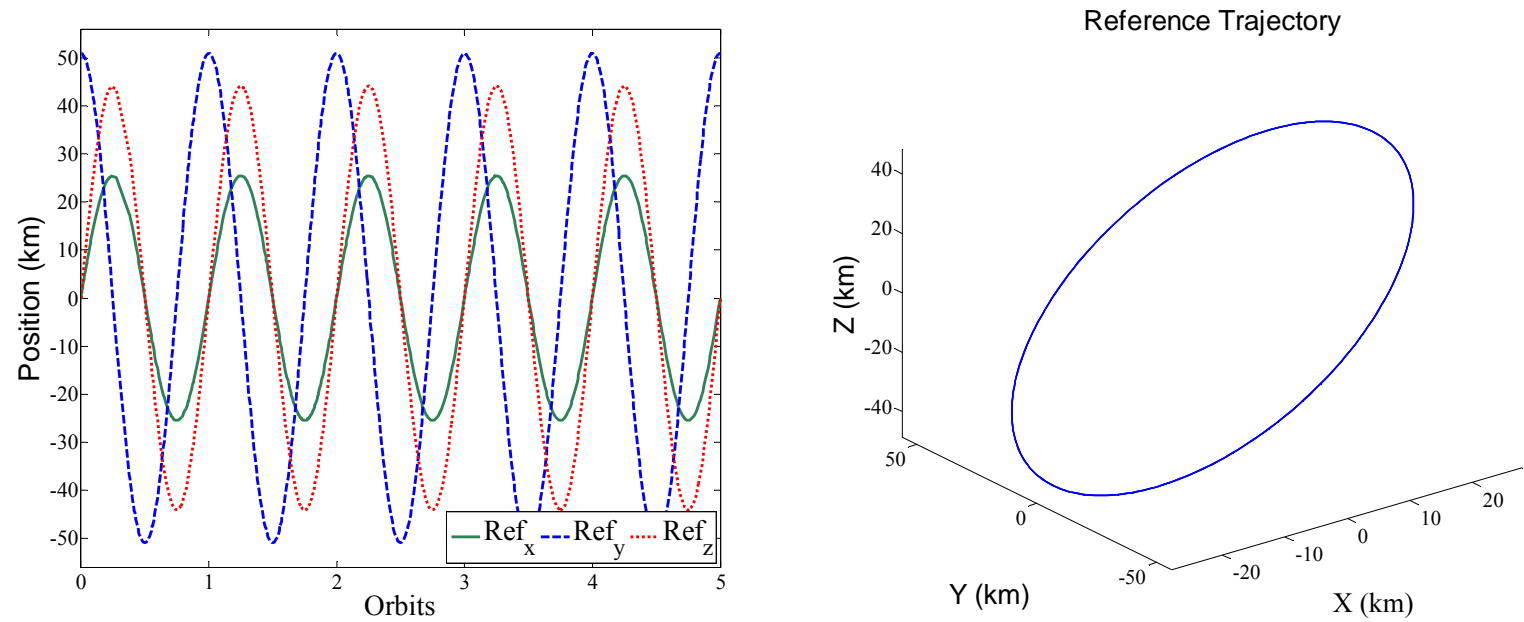

Figure 4.16 Reference trajectory

Even though it is said that the reference trajectory is going to be the same for all asteroids, the altitude of the reference trajectory is changed according to the dimension of the asteroid, and it is shown in Figure 4.16. Corresponding to the changes in the reference trajectory and the properties of the asteroid, the minimum input force is different than that of asteroid Castalia.

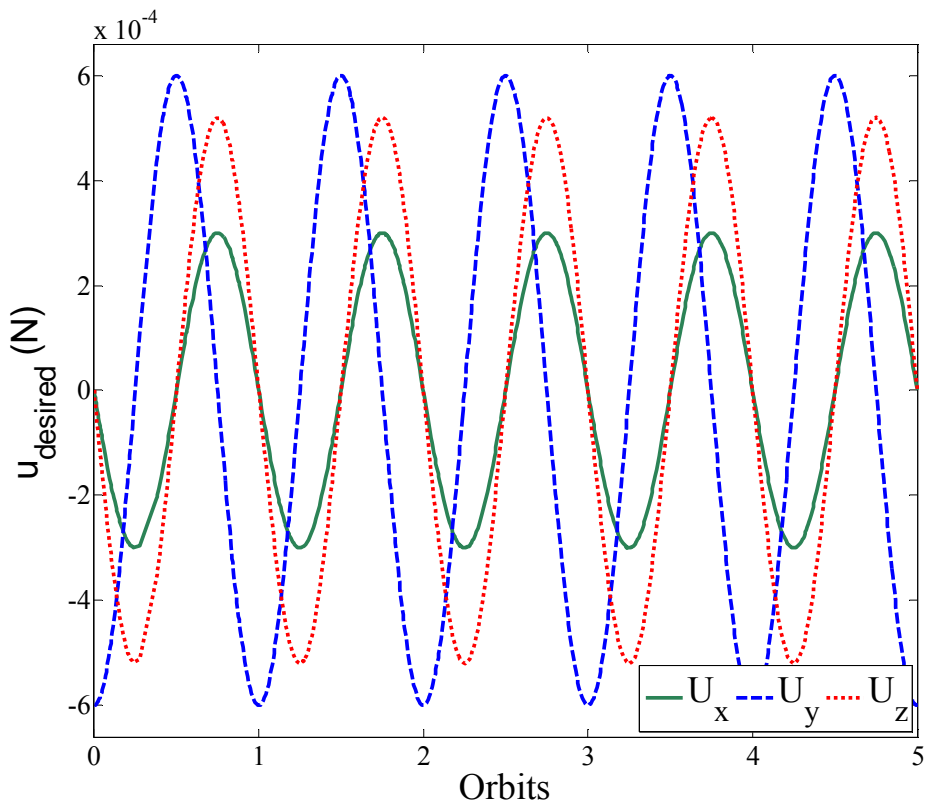

Figure 4.17 Minimum control input required (without disturbance)

The minimum system control input required is increased 10 fold as shown in Figure 4.17. Figure 4.18 is a snap shot of the formation as shown at different periods of time during the mission. 0.24 of an orbit is where the system experiences the actuator faults; therefore, the formation starts to be 
affected. At $2.8^{\text {th }}$ orbit, all 6 agents are far from the reference trajectory and try to recover both the tracking and

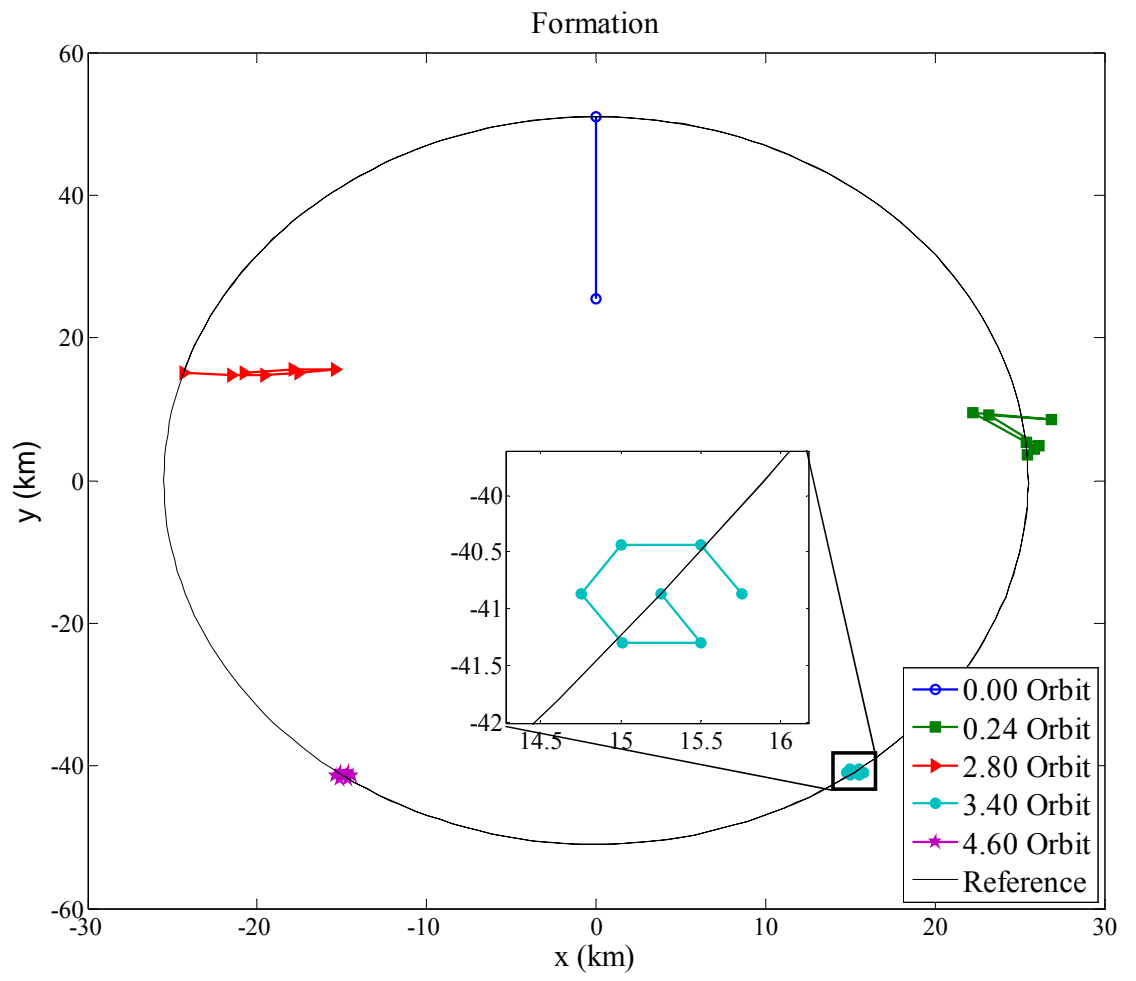

Figure 4.18: System state snap shot at different time

relative position errors. After 3.4 of an orbit, the formation is restored and it is back on track of the reference trajectory until the end of the mission.

The state of all agents and the state errors are shown in Figure 4.19 and Figure 4.20. The tracking error goes to zero slightly after the third orbit except for the z-direction. Compared with Castalia, the tracking error is greater in the y-direction than in the other two directions. As discussed in the previous section, there is no particular reasoning as far as the author's knowledge. 


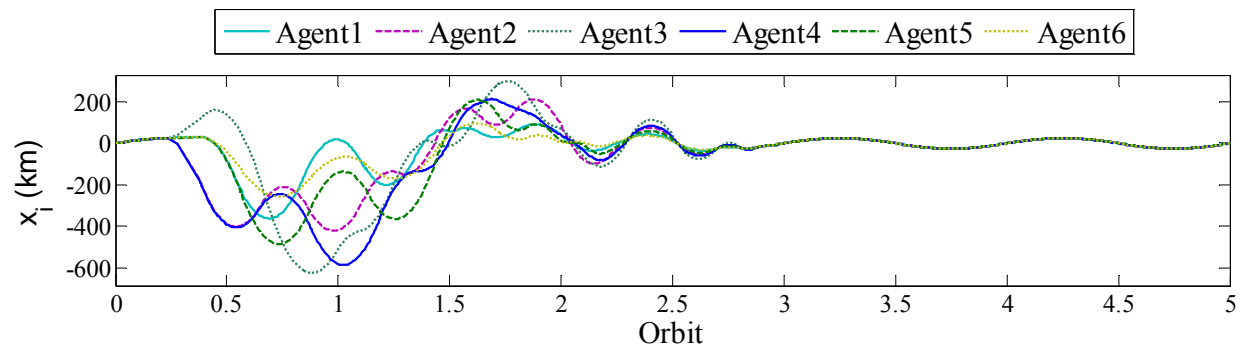

(a)

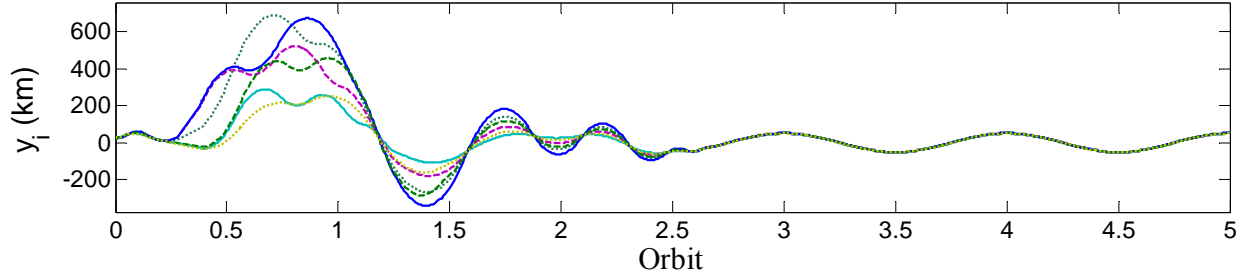

(b)

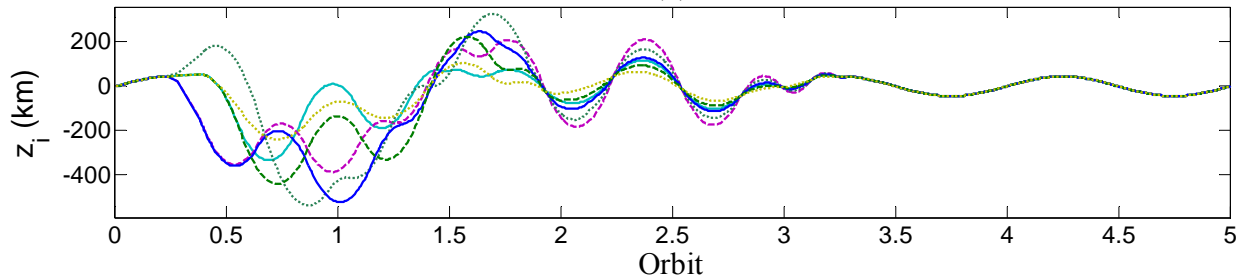

(c)

Figure 4.19: State of all agents', thruster malfunction
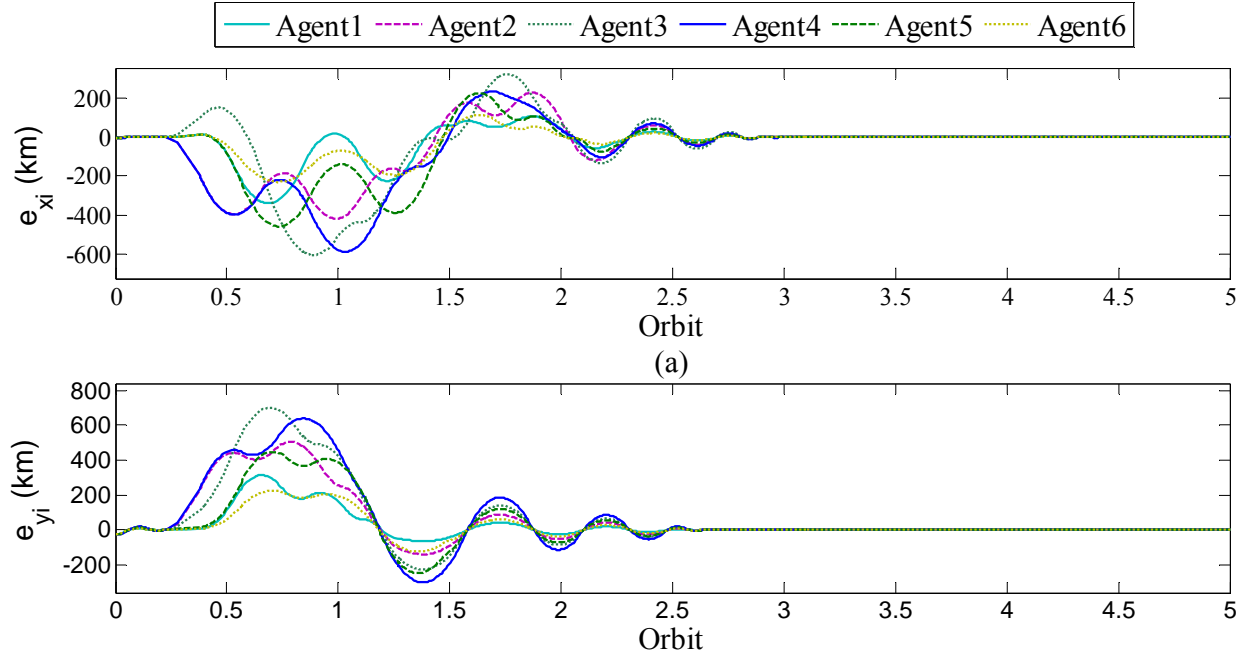

(b)

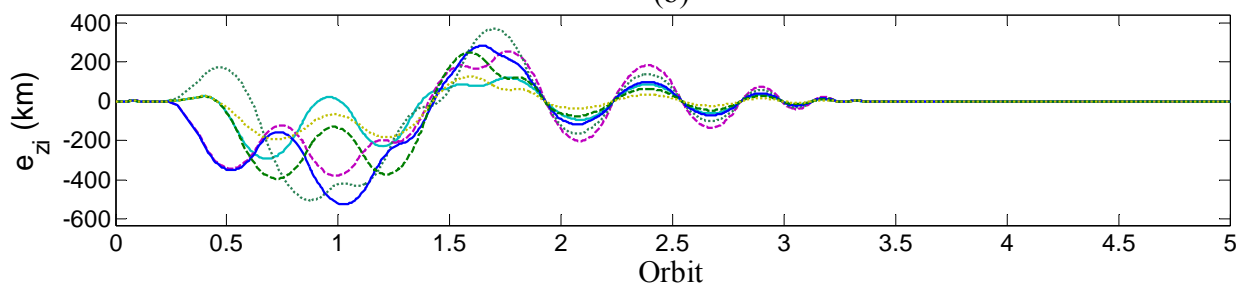

(c)

Figure 4.20: Tracking errors, all agents' thruster malfunction 

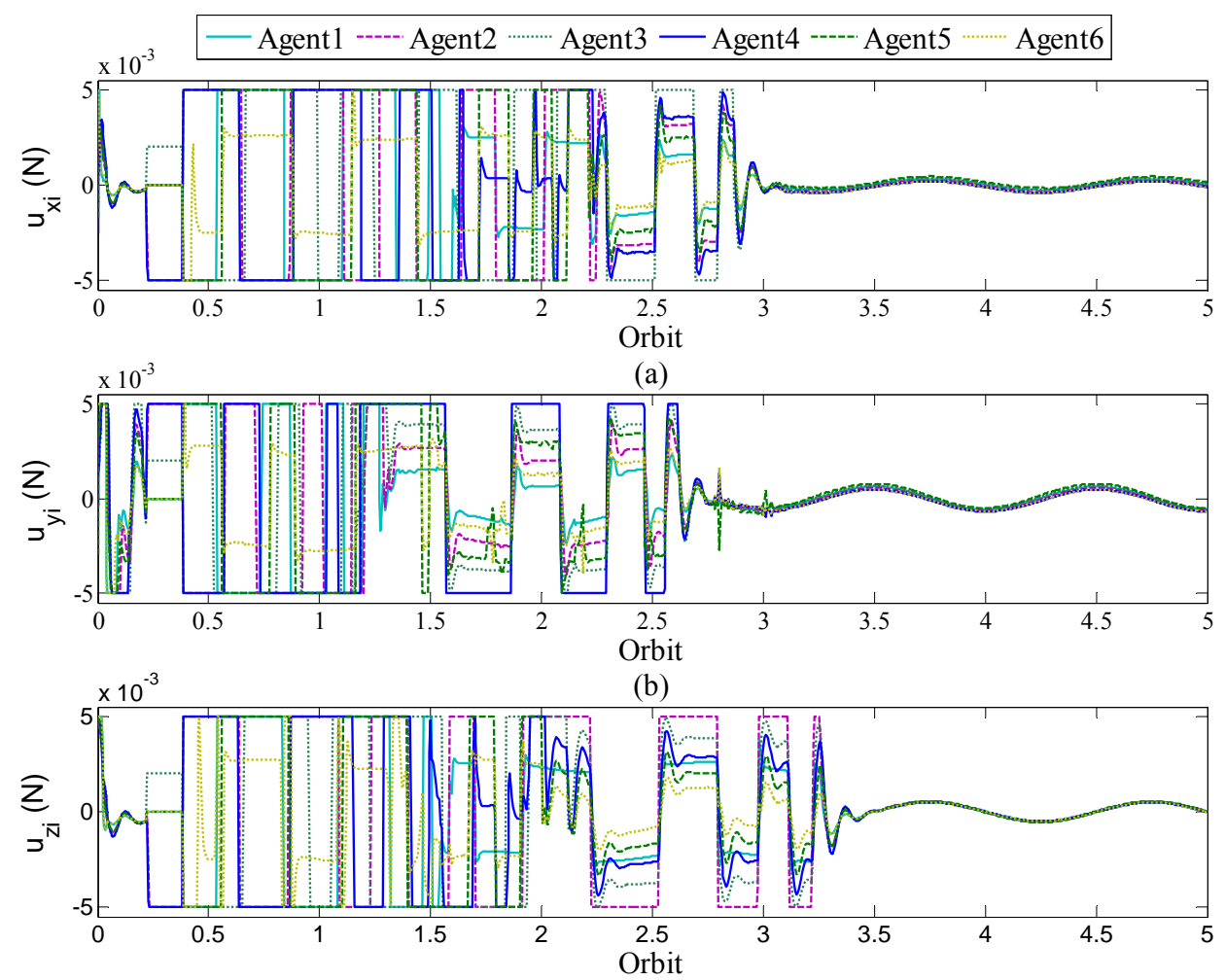

(c)

Figure 4.21: Control input, all agents' thruster malfunction

From Figure 4.21, it is shown that after the downtime, the thruster has reached its saturation state in order to control the system. It is clear that the system has reached its minimum input requirement at about 3.5 of an orbit. This indicates that the system is stable and controlled.

\subsubsection{243 Ida}

Asteroid Ida is a larger asteroid compared with 951 Gaspra. The mass of this asteroid is about 10 times heavier than that of asteroid Gaspra and its radius is almost double. However, it has a similar body spin rate with 4769 Castalia. Due to its geometric properties, it is expected that more control effort is required.

Since the spacecraft is flying at a different altitude, the elliptical reference trajectory for asteroid Ida is shown in Figure 4.22. The required input force for asteroid Castalia is $10^{-5} \mathrm{~N}$, and for asteroid Gaspra is $10^{-4} \mathrm{~N}$. The minimum thrust required is $1 \times 10^{-3} \mathrm{~N}$ for asteroid Ida, Figure 4.23 . 

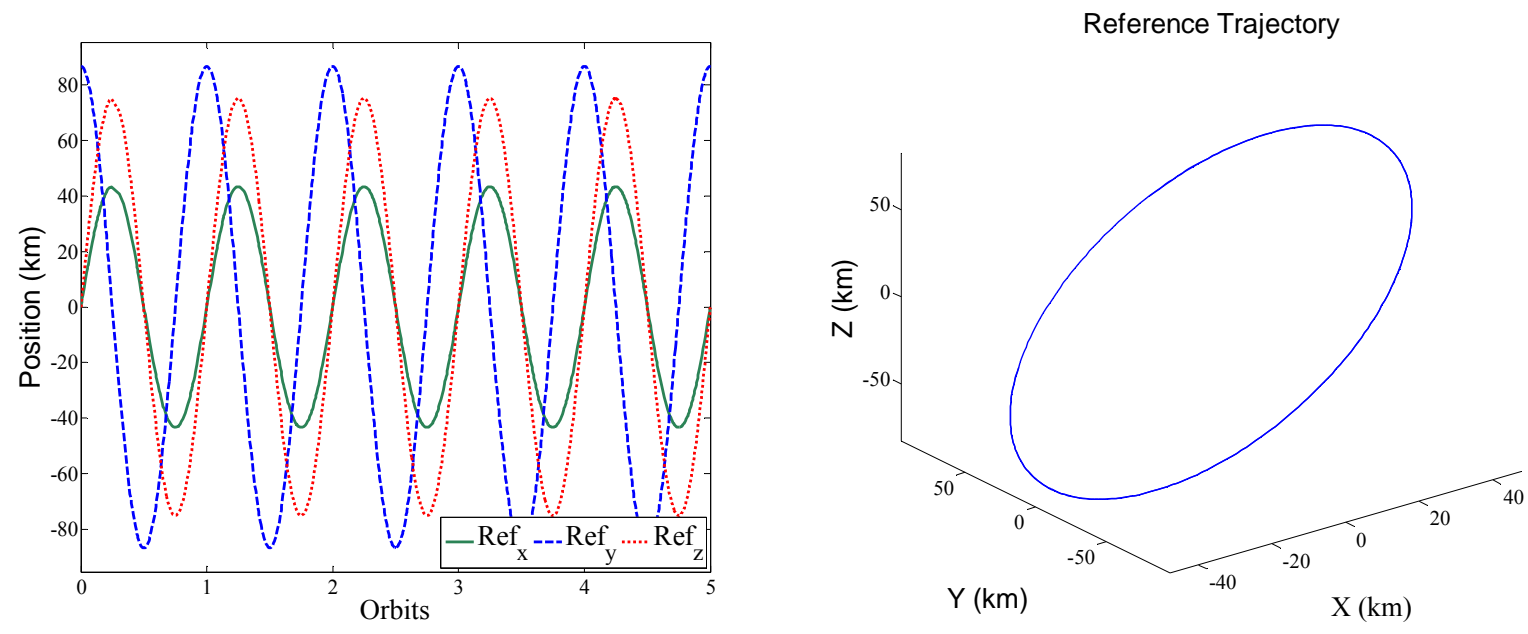

Figure 4.22 Reference trajectory

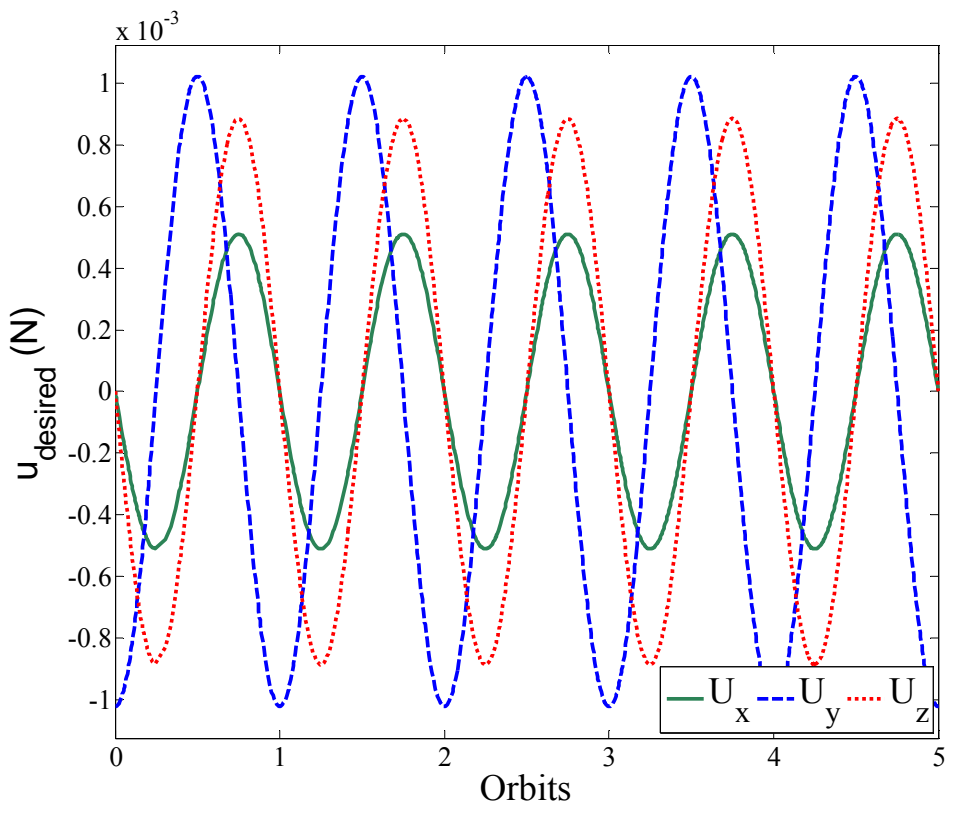

Figure 4.23 Minimum control input required (without disturbance)

In Figure 4.24, the formation of the swarm system is affected at 0.23 of an orbit. The downtime starts from 0.2184 of an orbit and ends at 0.3822 of an orbit. After this downtime, the spacecraft are not able to reinstate back to the reference trajectory until 3.05 of an orbit. As seen, even though the tracking error has been reduced, the formation does not form as defined at 3.88 of an orbit. 


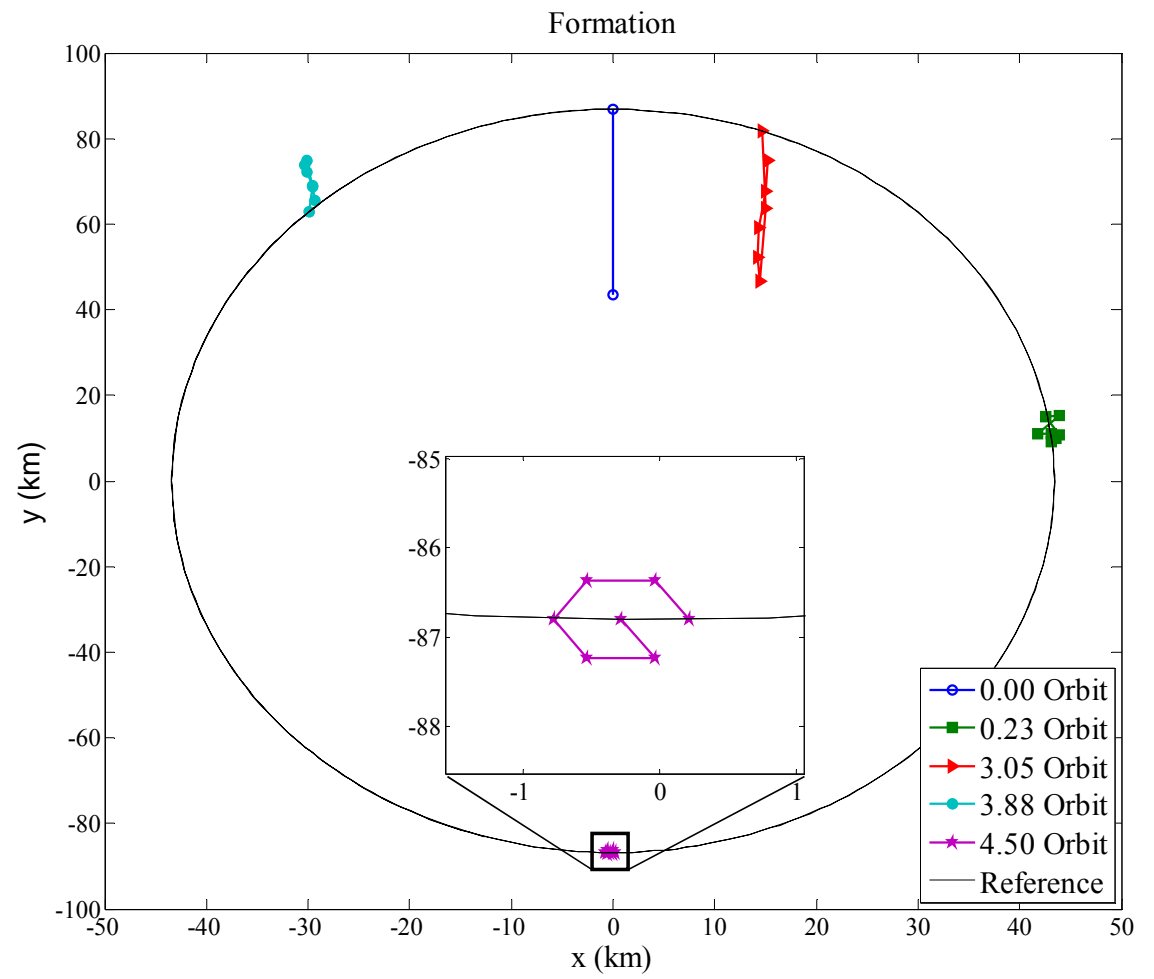

Figure 4.24: System state snap shot at different time

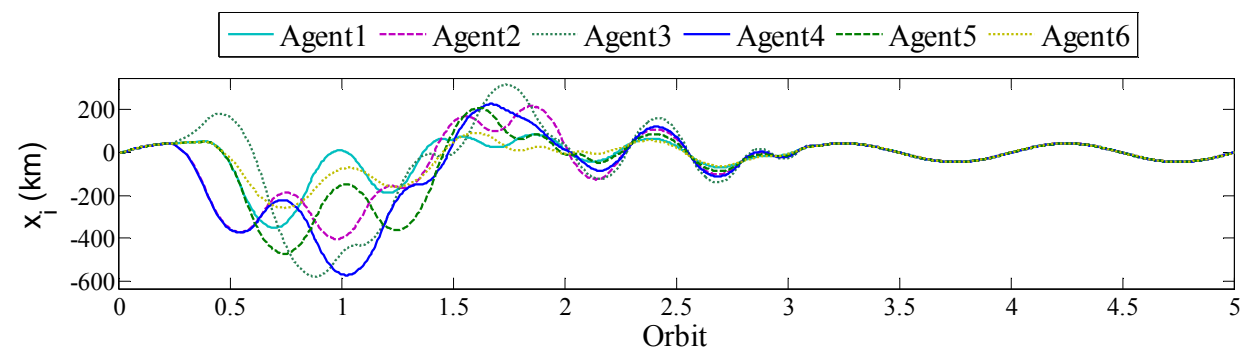

(a)
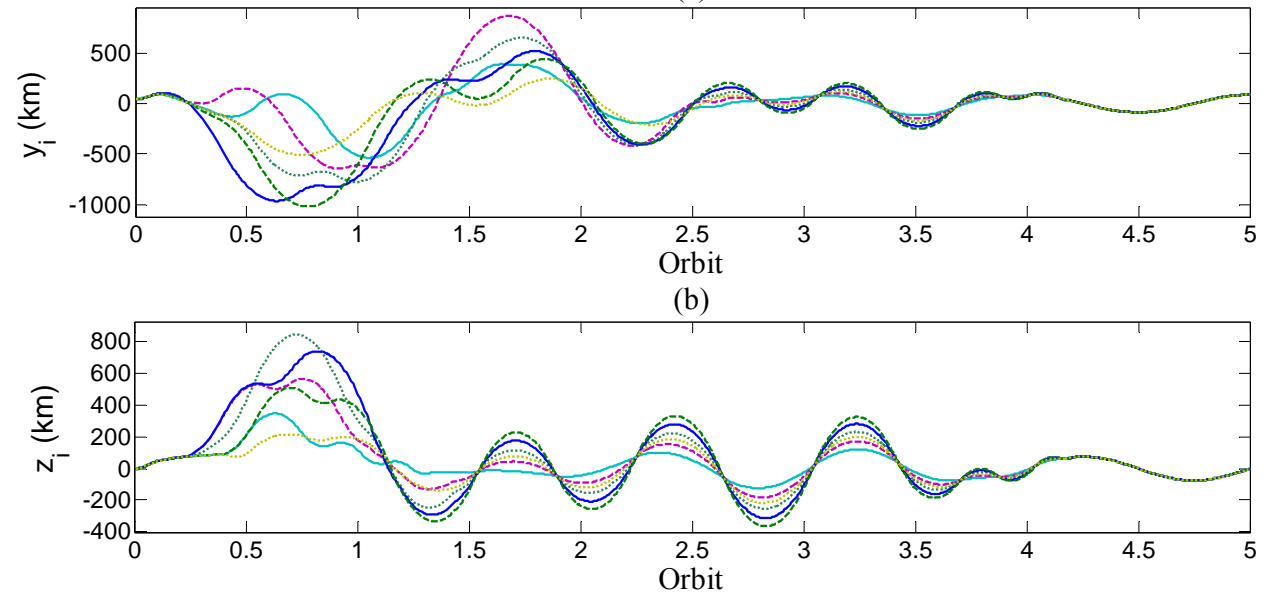

(c)

Figure 4.25: State of all agents', thruster malfunction 
Comparing Figure 4.25 and Figure 4.26 with the results calculated for asteroid Gaspra, there are significantly more state errors in the z-direction. This continues to support that there are no obvious reasons for why a specific tracking error is more dominating in a single direction.
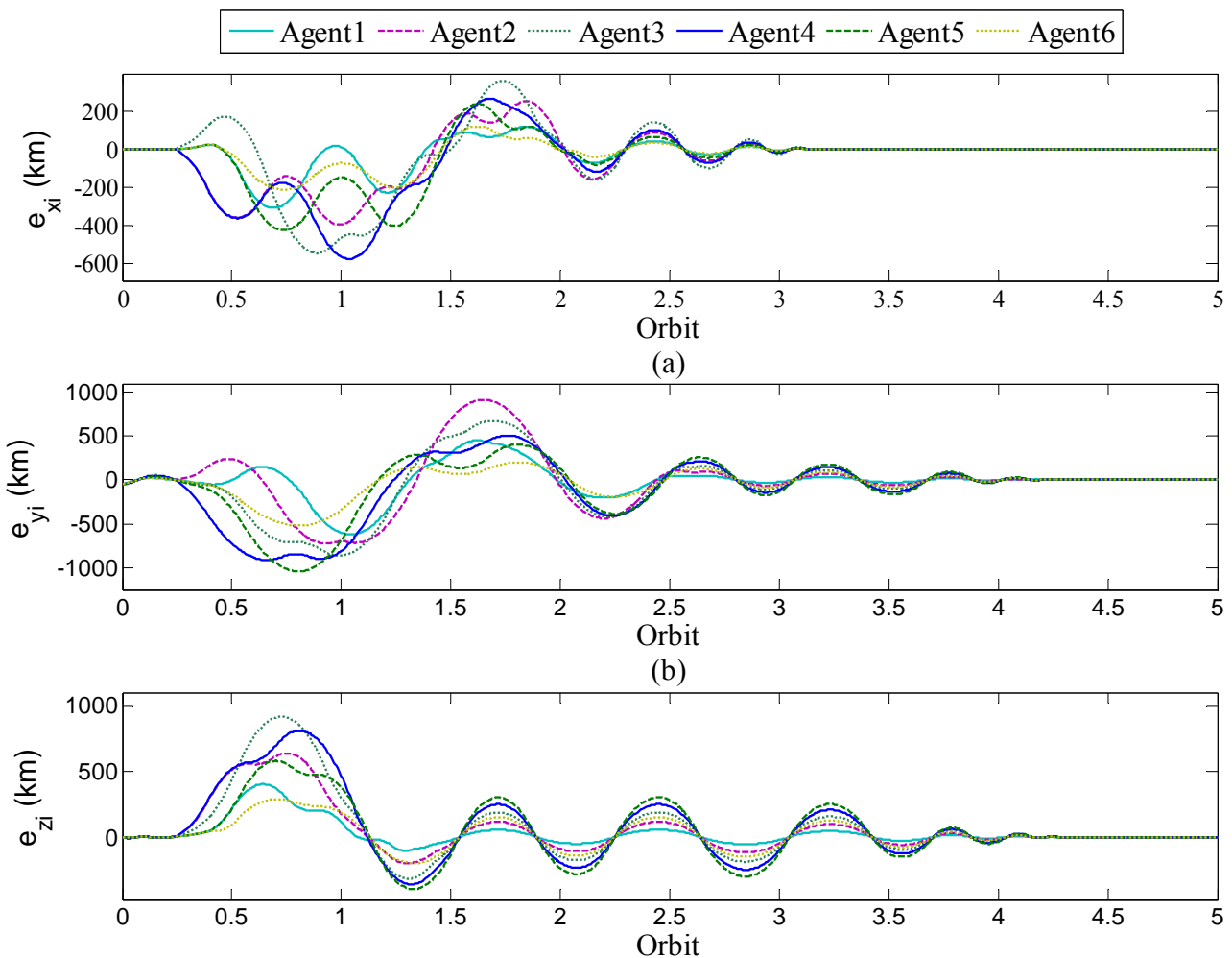

(c)

Figure 4.26: Tracking errors, all agents’ thruster malfunction

As brought up from Figure 4.23, the minimum system requirement is $1 \mathrm{mN}$, and the maximum thrust provide is $5 \mathrm{mN}$. It is expected to take longer to stabilize the system. In Figure 4.27, the system stays at its thrust saturation much longer than the mission for asteroid Gaspra and Castalia. This could be the reason why, in this case, even the $\mathrm{z}$ direction is experiencing higher system state errors. 

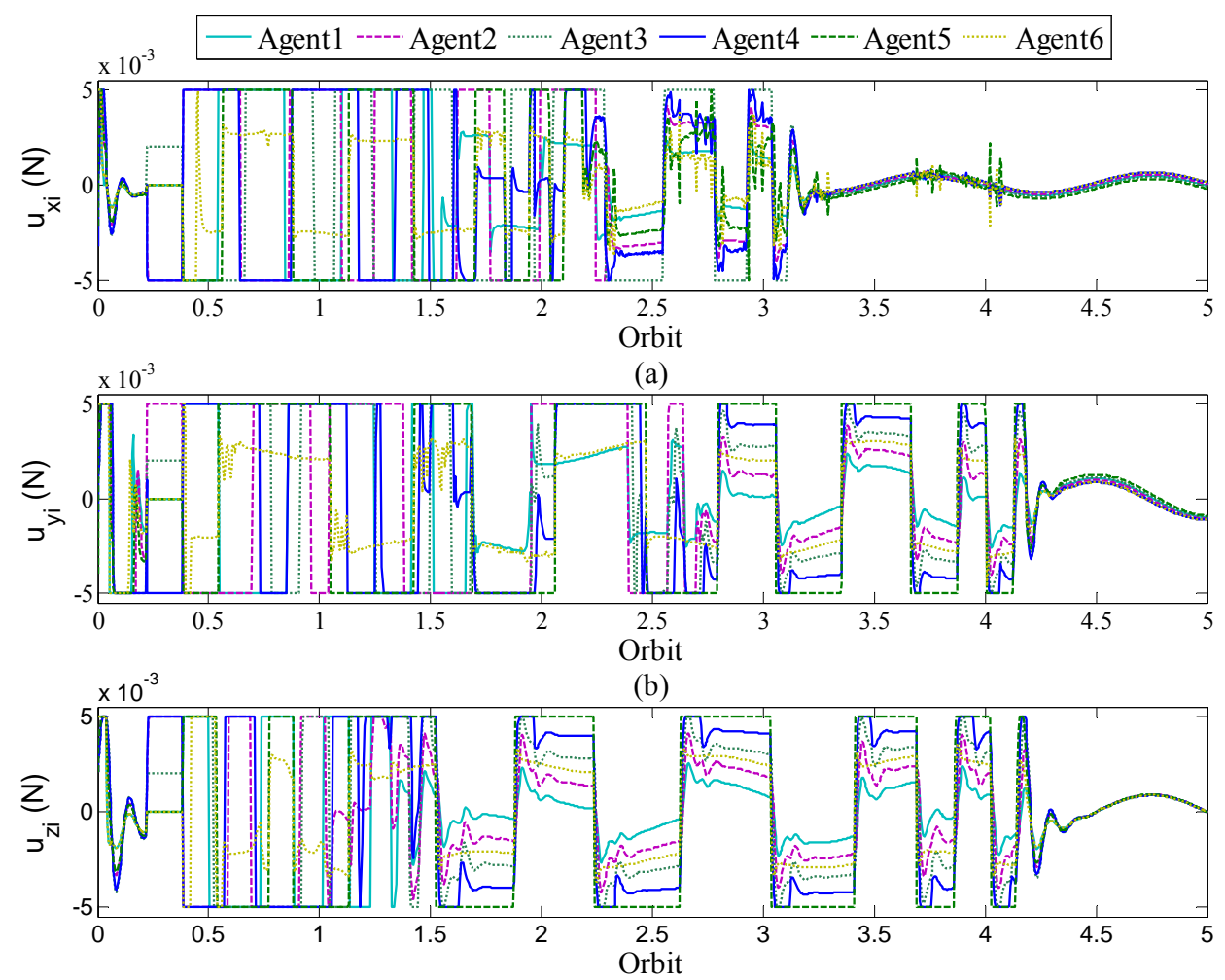

(c)

Figure 4.27: Control input, all agents’ thruster malfunction

These results indicate that the control law is able to guide the system to accomplish the mission even when 5 minute multiple actuator faults are put into operation.

\subsubsection{4 Vesta}

Out of all four asteroids, asteroid Vesta has the most challenging physical geometry. Both the mass and the radius of the asteroid are the largest among the four. However, the spin rate of this astronomical body is in between Gaspra and Ida. With the largest radius comes the highest altitude, also with such heavy weight, the gravitational force is much more significant. This phenomenon is reflected in Figure 4.28 and Figure 4.29. The minimum required force is $15 \mathrm{mN}$, the $5 \mathrm{mN}$ saturation does not work for this asteroid. With the specified reference trajectory, a nano scale spacecraft is not be able to orbit around this asteroid. Therefore, a $50 \mathrm{mN}$ maximum thrust is given in this scenario. 

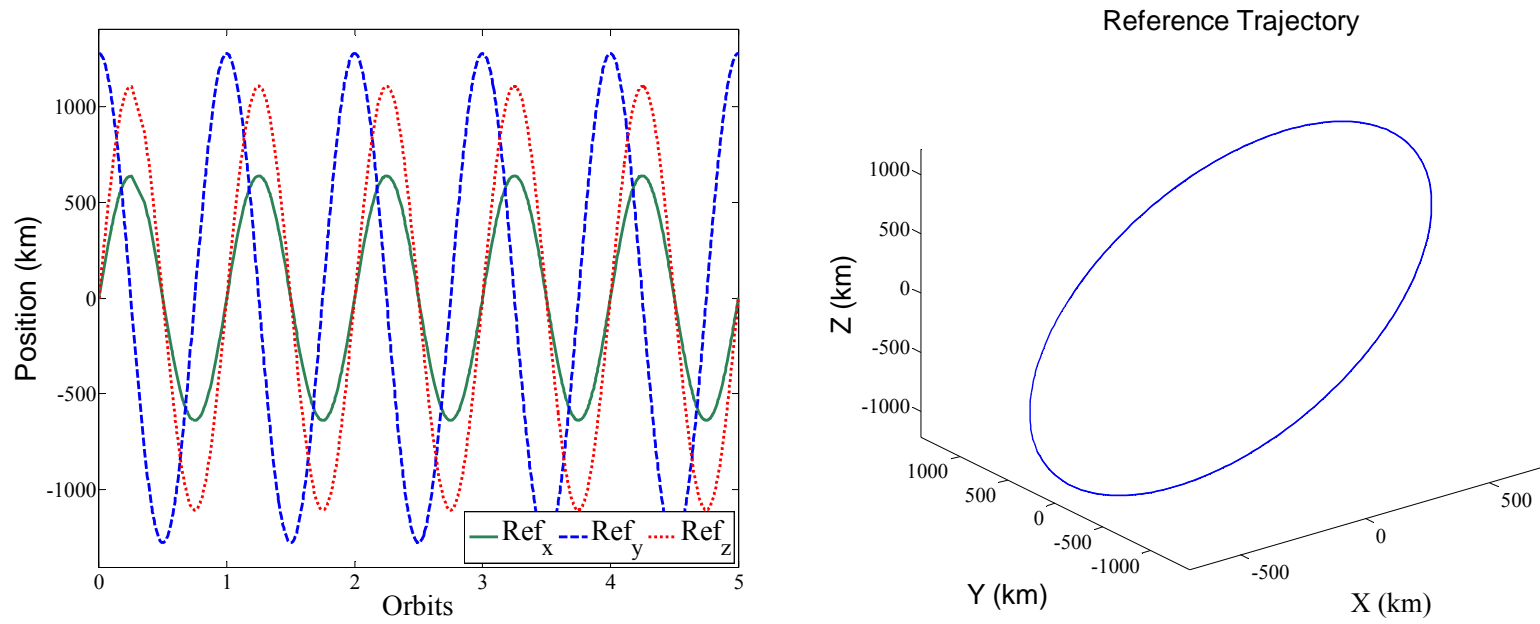

Figure 4.28 Reference trajectory

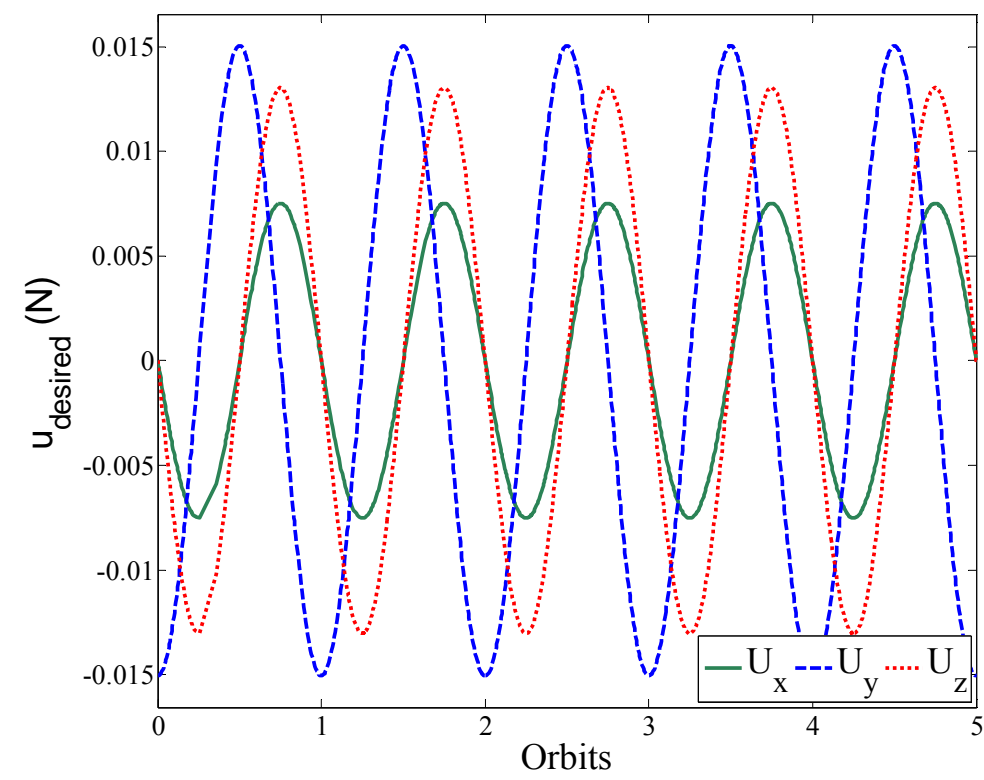

Figure 4.29 Minimum control input required (without disturbance)

The state of the spacecraft is shown in Figure 4.30 and Figure 4.31. A colossal state error is the result of the excessive amount of thrust provided by the actuator. Agents 2 and 4 are having a reversal sign actuator fault that can rise up to $50 \mathrm{mN}$. In a short 5 minutes, the position of the spacecraft has increased from a 1,280 km to almost $8,000 \mathrm{~km}$ in the $\mathrm{x}$-direction, and it has gone to nearly $10,000 \mathrm{~km}$ in the z-direction, which is also shown in the state error graph, Figure 4.32. 


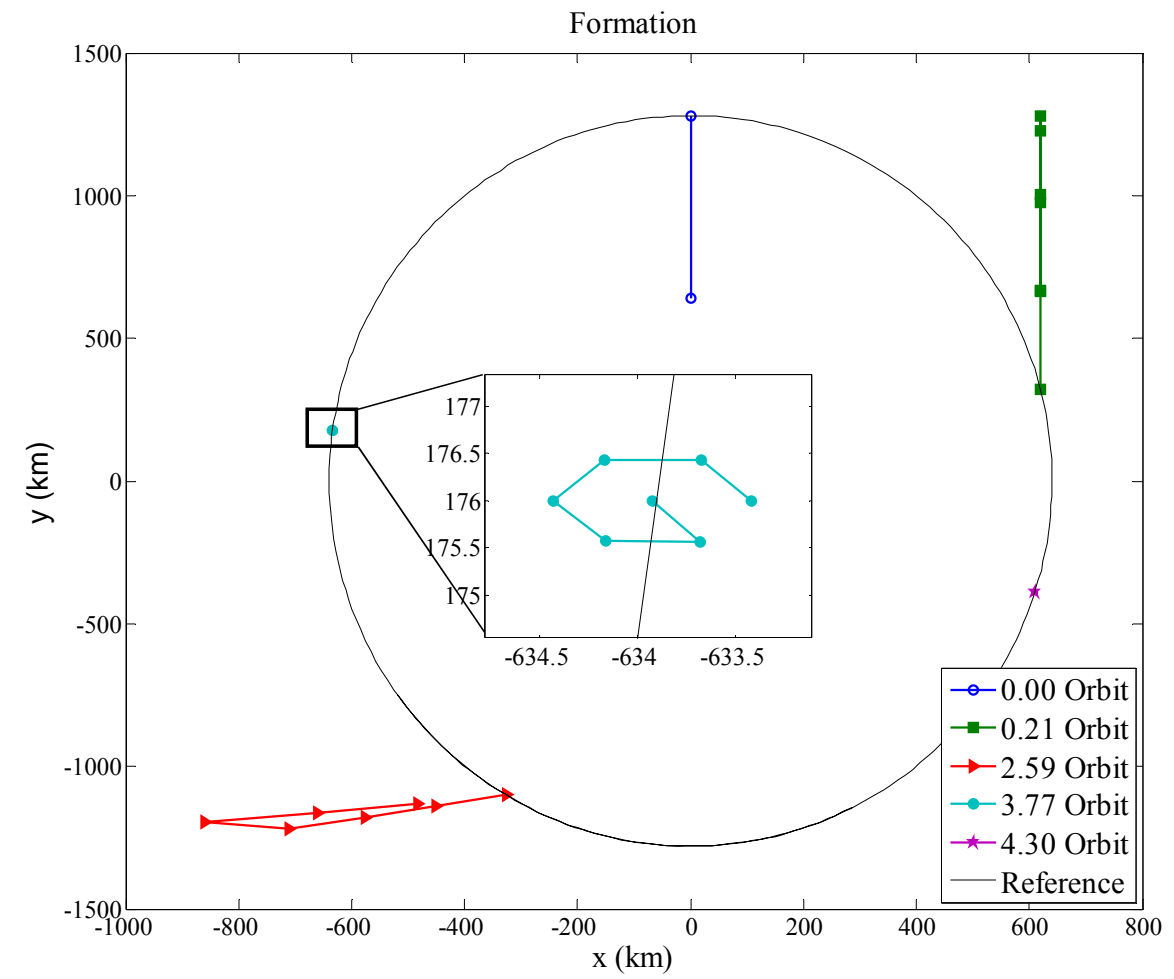

Figure 4.30: System state snap shot at different time

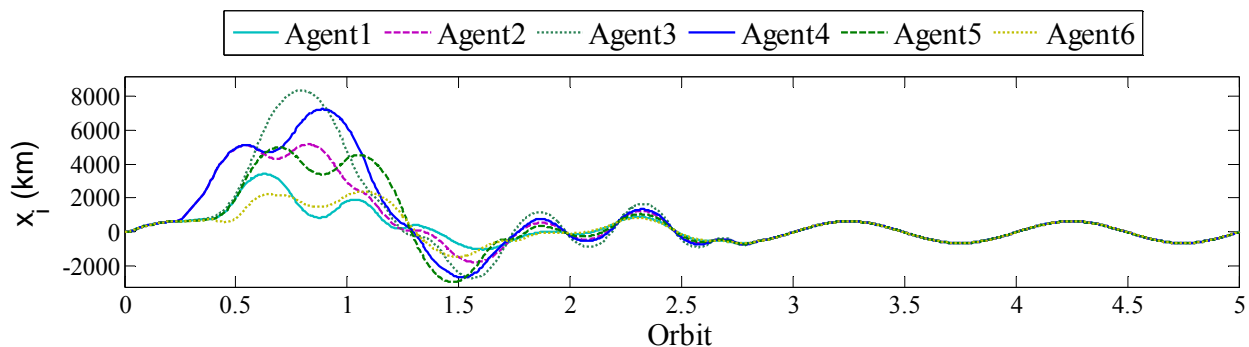

(a)

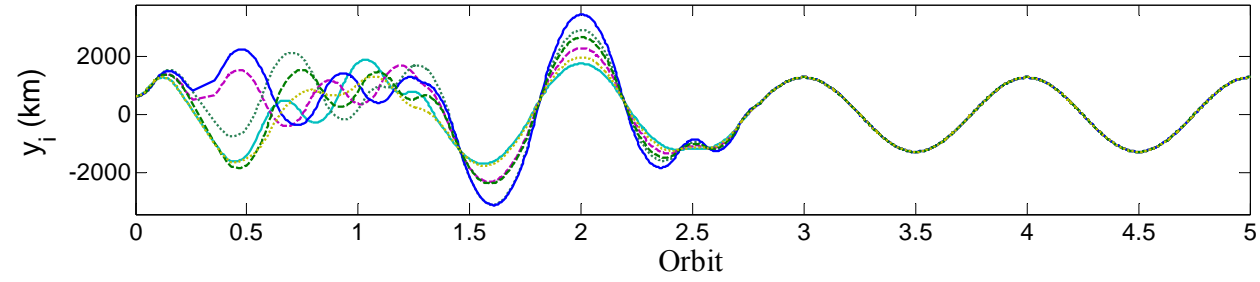

(b)

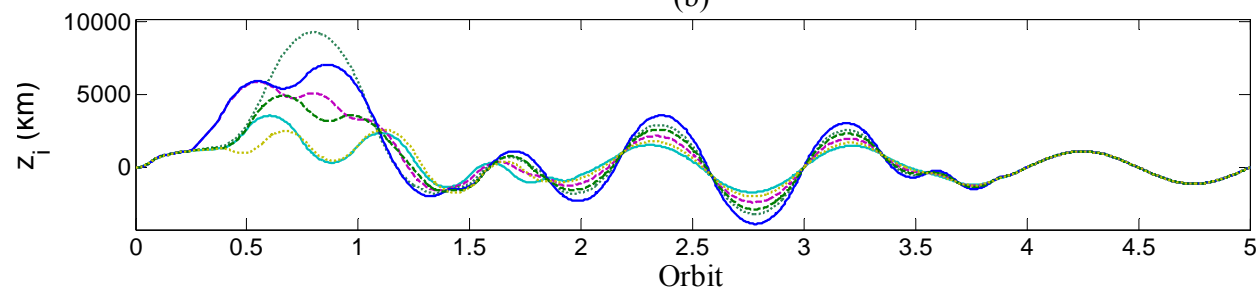

(c)

Figure 4.31: State of all agents', thruster malfunction 


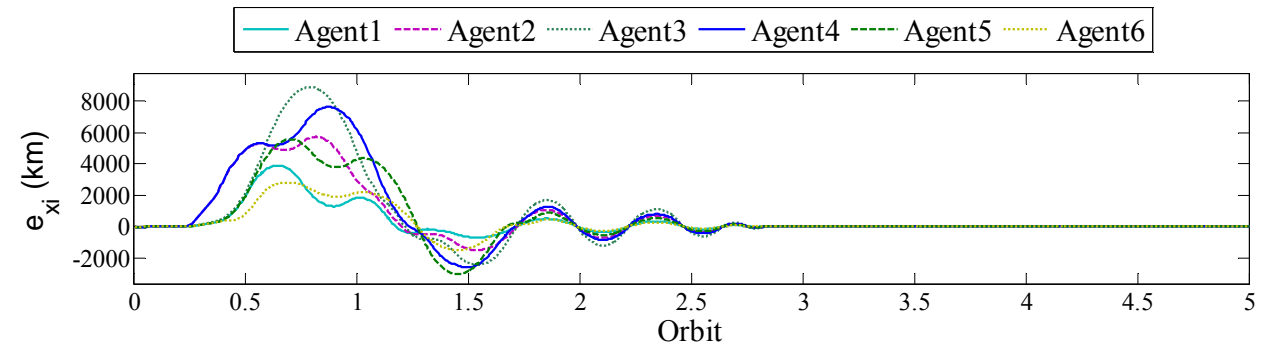

(a)

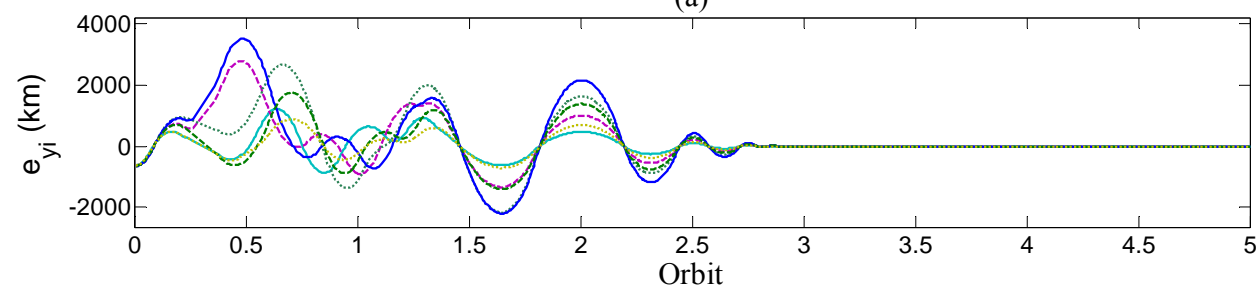

(b)

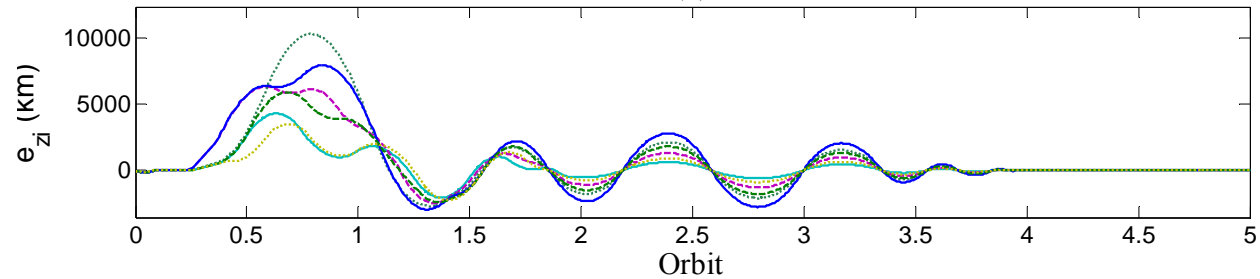

(c)

Figure 4.32: Tracking errors, all agents' thruster malfunction
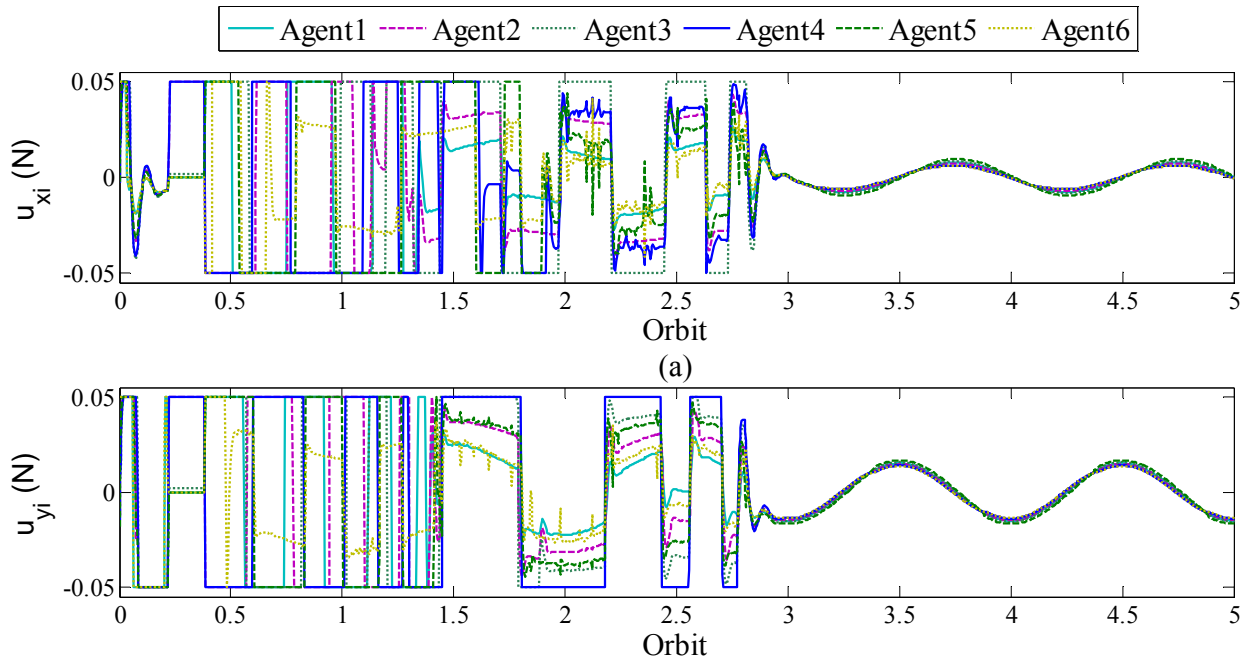

(b)

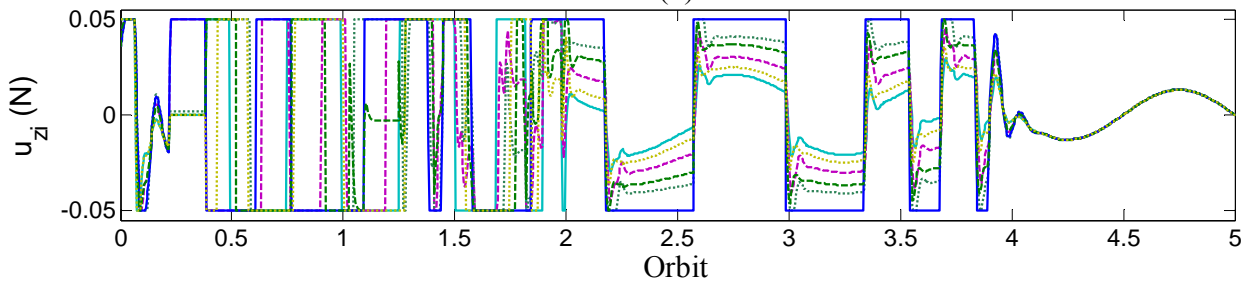

(c)

Figure 4.33: Control input, all agents' thruster malfunction 
It is studied in Chapter 4.2.3 and it is verified in Figure 4.33, that with excessive amount of thrust, the system is controlled. However, during the downtime, depending on the type of actuator fault that is examined, it might bring a large amount of state error, which requires even more thrust in maintaining the agents in the right configuration and correct position.

\subsubsection{Effect of Spacecraft Mass Variations}

Pursuing the discussion from Chapter 3.2.5, the similar phenomena is examined again. In a mission, the mass of the spacecraft is a very important parameter. Often the mass of the spacecraft might change when the control law is designed. Moreover, the mass of the spacecraft decreases during the mission when propellant is used. Therefore, it is a good control law if the control parameter chosen is stiff enough to allow the control system to work within a certain interval of spacecraft mass. Previously, a 2, 5, and $10 \mathrm{~kg}$ spacecraft mass were examined. In this Chapter, a 2 and $10 \mathrm{~kg}$ spacecraft is examined again, since $10 \mathrm{~kg}$ was not very successful in the previous control law. The last scenario that is added is where the mass of the spacecraft varies during the mission. The mass of the spacecraft reduces from $10 \mathrm{~kg}$ to $1 \mathrm{~kg}$ in half of the period of a reference orbit. The mass of the spacecraft decreases at a constant speed within a specific time. It is a good model simulating that the propellant is burned from the beginning of the mission and is done within half of the reference orbit.

The first scenario, when the mass is close to $1 \mathrm{~kg}$ the robust SMC control law has a very similar effect as the SMC. The state error, Figure 4.34, has a very similar profile as Figure 3.35. The control input for the robust SMC is shown in Figure 4.35, which is comparable with the minimum system control input required, Figure 2.7.

Figure 4.36 and Figure 4.37 are the absolute error metric and the relative error metric of the system when the mass of the spacecraft is set to be $10 \mathrm{~kg}$. The relative error converged to zero, which means that consensus formation has been achieved. However, the total tracking error for all 6 agents is approximately $0.3 \mathrm{~km}$. In average, each agent is about $0.05 \mathrm{~km}$ away from the desired trajectory. The control effort for this scenario is very similar to the one given for $2 \mathrm{~kg}$. When the mass of the spacecraft decreases from $10 \mathrm{~kg}$ to $1 \mathrm{~kg}$, the error of the system reduces to zero. It is shown in the AEM and REM, Figure 4.38 and Figure 4.39, respectively. 


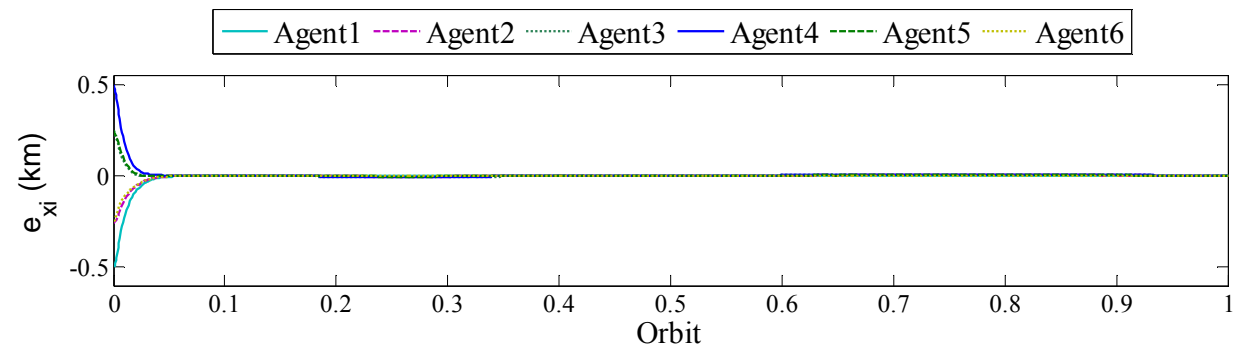

(a)

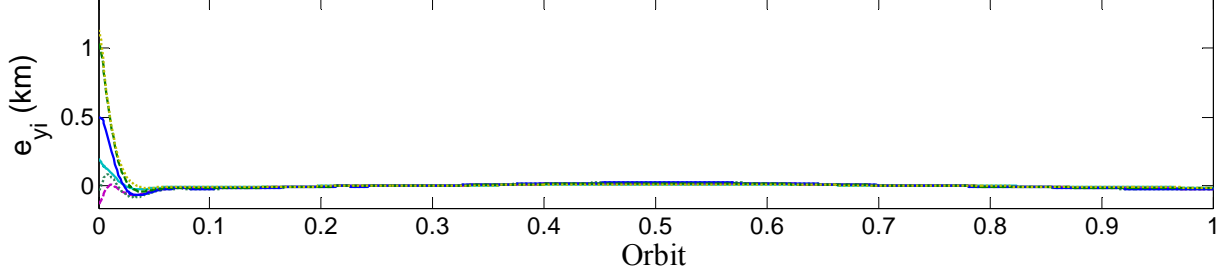

(b)

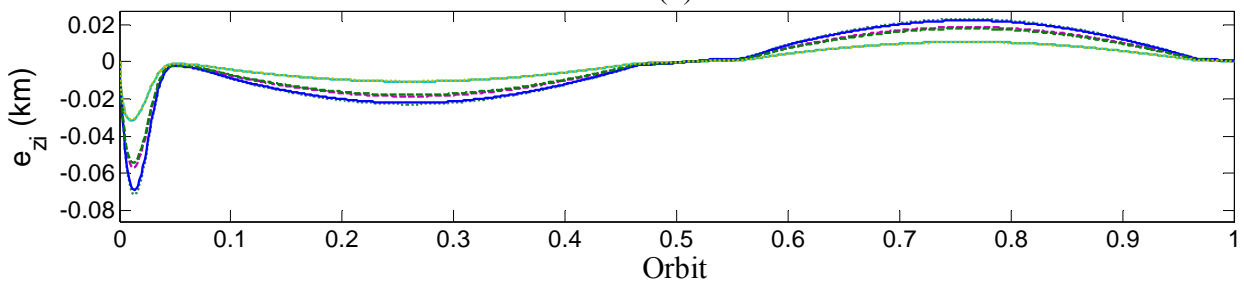

(c)

Figure 4.34: Tracking errors, spacecraft mass $=2 \mathrm{~kg}$
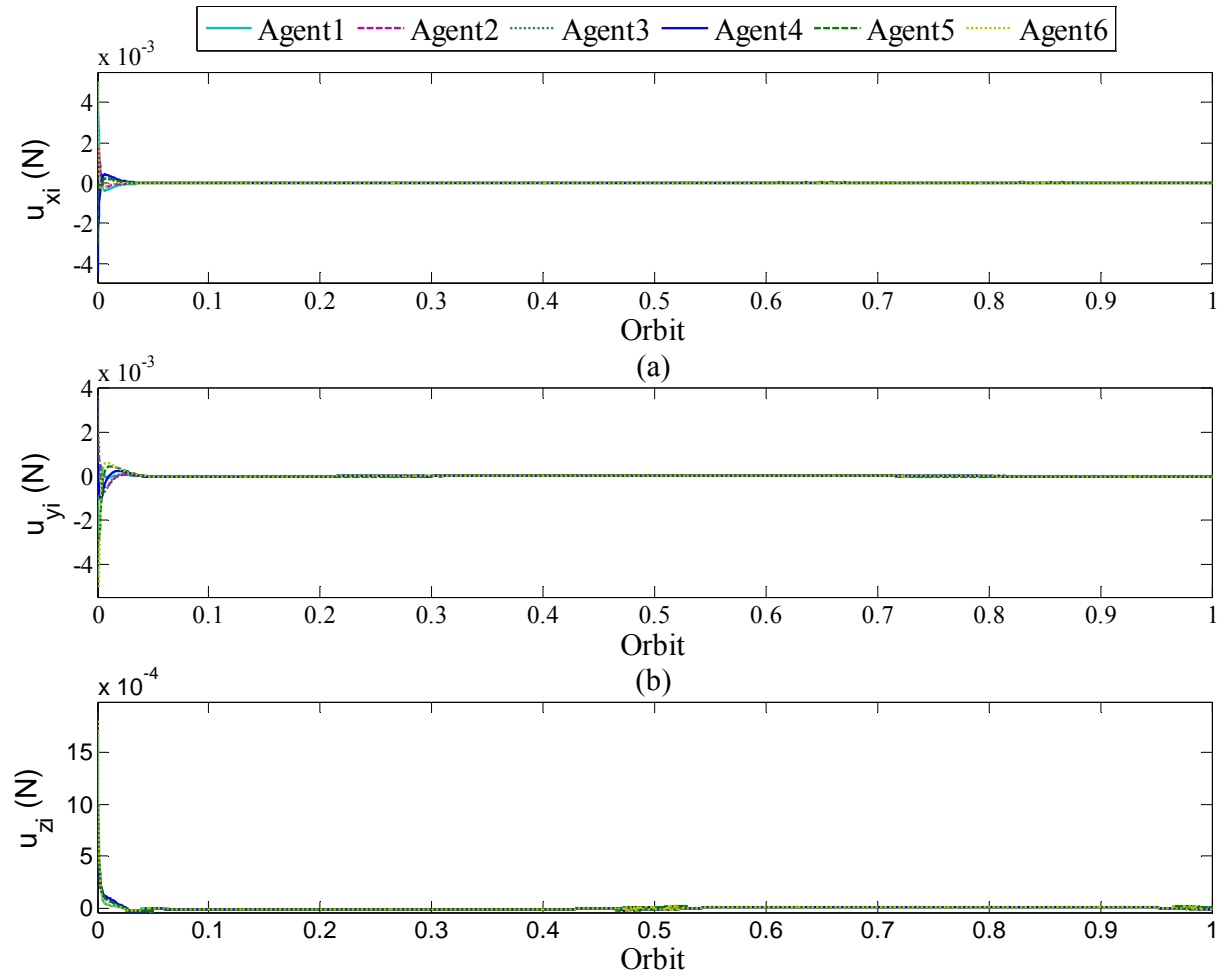

(c)

Figure 4.35: Control input, spacecraft mass $=2 \mathrm{~kg}$ 


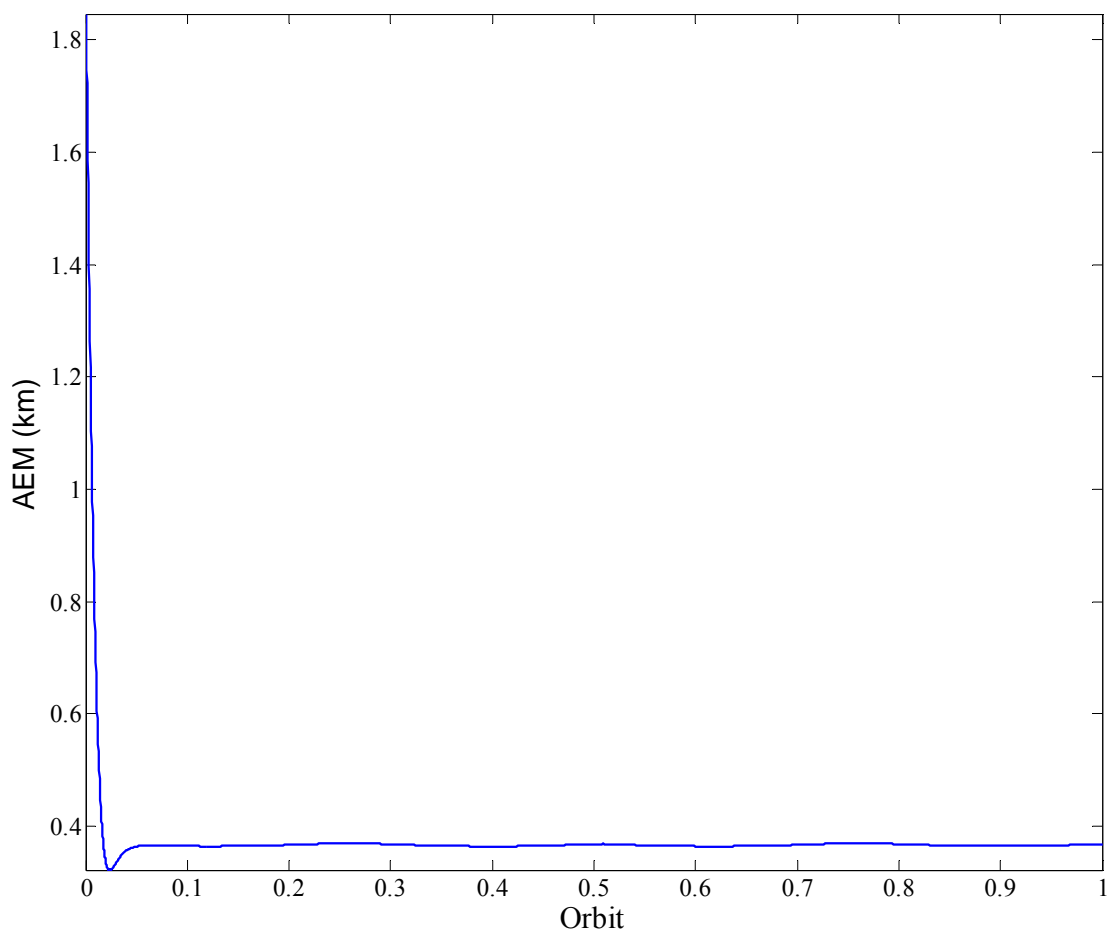

Figure 4.36: AEM, spacecraft mass $=10 \mathrm{~kg}$

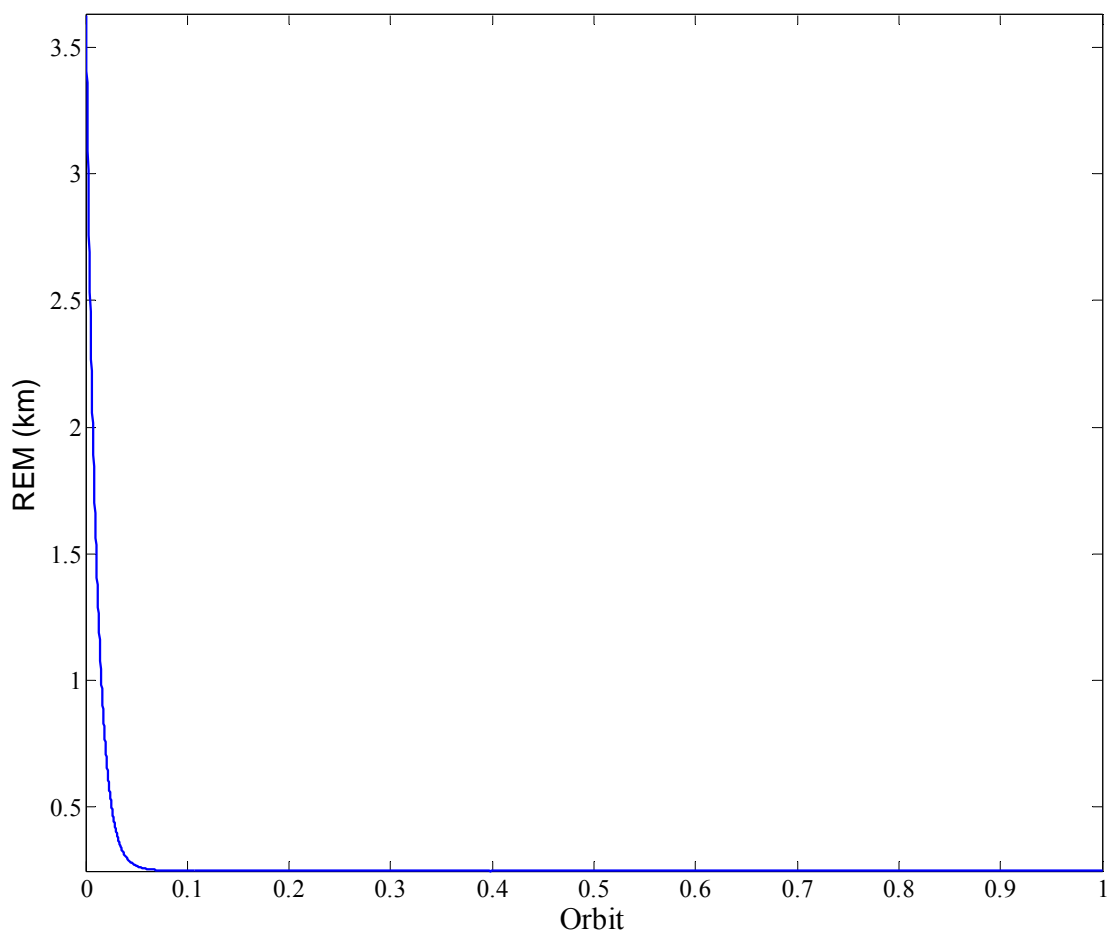

Figure 4.37: REM, spacecraft mass $=10 \mathrm{~kg}$ 


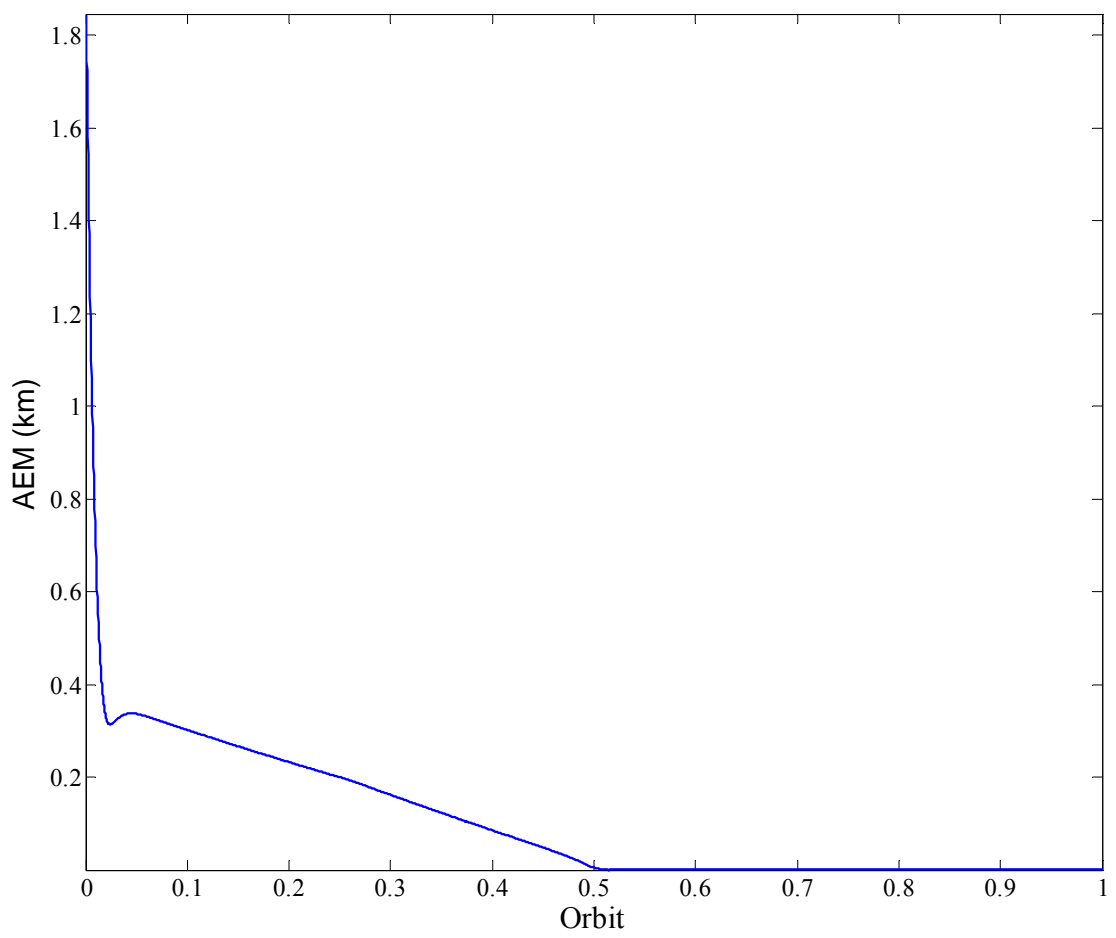

Figure 4.38: AEM, spacecraft mass reduce from $10 \mathrm{~kg}$ to $1 \mathrm{~kg}$

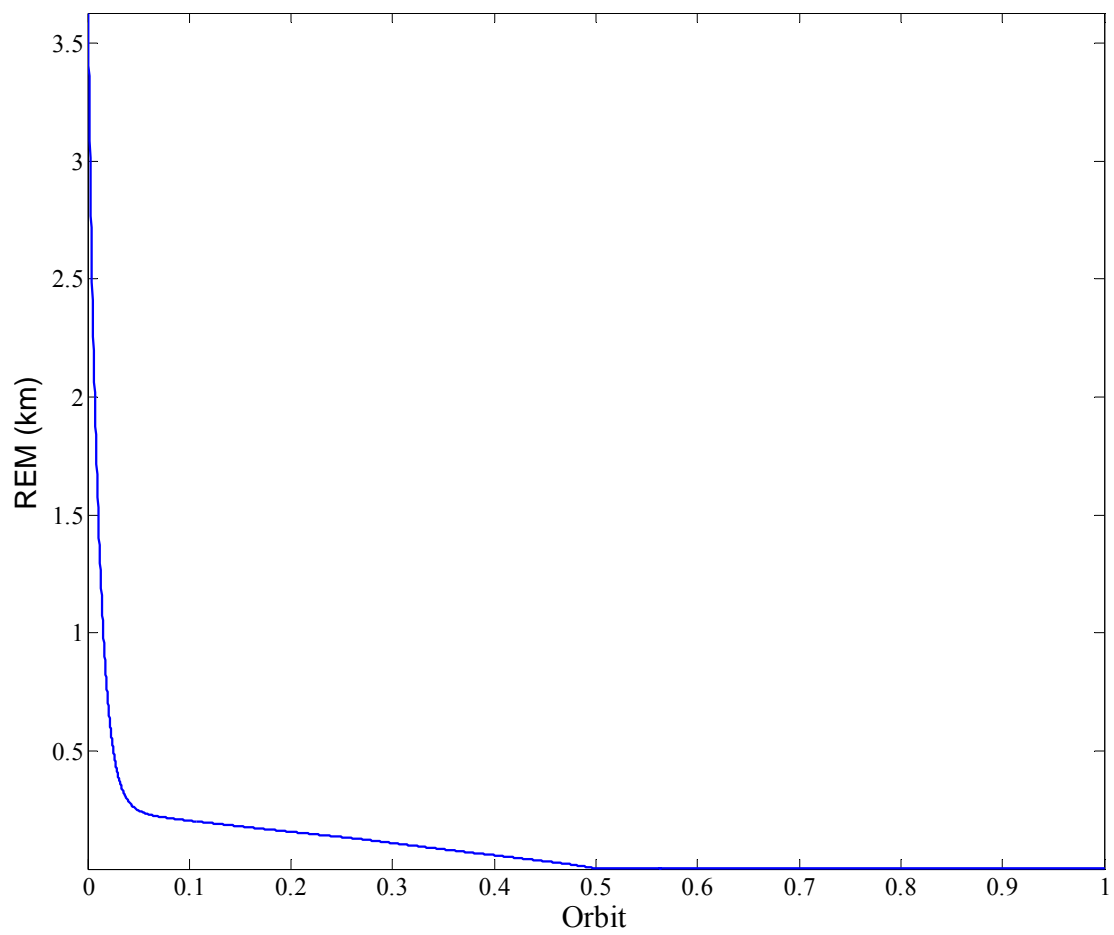

Figure 4.39: REM, spacecraft mass reduce from $10 \mathrm{~kg}$ to $1 \mathrm{~kg}$ 
The dynamic system and parameters used in this simulation are the same as the ones proposed in Chapter 3. The reference trajectory, as well as the control gains are all the same in order to make a reasonable comparison. With the use of the robust SMC, there is an improvement in the system error. The last scenario is the best model for a nano spacecraft, where it starts with a maximum takeoff weight, during the mission, mass decreases gradually to the payload mass. With the chosen control parameters, the robust SMC is able to control all 6 spacecraft where the mass is different from the mass that is given in the system model.

\subsection{CONCLUSIONS}

In this chapter the robust control law is used in the consensus formation flying system. The numerical simulations show that the performance is improved when downtime is introduced to the system. The sliding mode control developed in Chapter 3 was capable of stabilising the system with the proposed downtime. However, the system reaches its desired state faster for both before and after the downtime with robust control. This reduces the large AEM and REM of the mission but increase the control input. It is a well known paradox in the control literature that in order to achieve robustness, some part of performance has to be compromised. Various asteroids are examined in this chapter. The proposed control law works for the mission exploring asteroid Gaspra, Ida, and Vesta. These asteroids are chosen in a way that each one of them has a special geometry or dynamic challenge. Lastly, the proposed control law works for a specific range of spacecraft masses, and it is examined with the case where the mass of the spacecraft varies during the mission.

In the next chapter a terminal sliding mode control (TSMC) is applied and shows considerable improvement of the SMC and robust SMC. Furthermore, an adaptive control topology, the Chebyshev neural network $(\mathrm{CNN})$, is developed to model uncertain mission dynamics as is best suitable for this highly unstable asteroid mission. Robust control is also applied to make the controller more robust for the existences of estimation error. 


\section{NEURAL NETWORK ADAPTIVE CONTROL OF CONSENSUS SPACECRAFT FORMATION FLYING}

Neural network approximation together with adaptive control techniques have been proposed by a number of investigators for controlling nonlinear plants with unknown dynamics. Chebyshev polynomial is applied in order to learn the unknown dynamics. Chebyshev neural networks $(\mathrm{CNN})$ use this polynomial to approximate the unknown nonlinear system states and feedback the estimated value to the controller. In this chapter, the orbital dynamics of an asteroid is unknown. Chebyshev neural networks are applied to approximate these unknown dynamics. For the proposed control law, the neural network is combined with robust control and adaptive control. The control technique applied in this control law is Terminal Sliding Mode Control (TSMC) and the weights of the neural network are adjusted on-line. Furthermore, this nonlinear control algorithm is formulated with a detailed proof of stability for the closed-loop system by the Lyapunov theorem. Moreover, the results of numerical simulations incorporating different fault scenarios are presented for a detailed assessment of the system performance under the proposed control strategies and validation of the established theoretical framework. Finally, some brief conclusions are provided.

\subsection{Design of CONTROL LaWs}

Literature review shows that most existing consensus controls for multi-agent systems have assumed that the dynamic states of each agent are known. In this chapter the control law is constructed in a way that the dynamics of the system are omitted, in order to model the uncertain dynamic scenario. Neural networks systems have been applied to various applications that can be used to approximate any smooth functions. The preceding control law with the enhancement from the Chebyshev neural networks is then developed to bring out the uncertain dynamics and the bounded external disturbances. Due to the complexity of the new control law a terminal sliding mode control is applied to achieve a faster convergence and higher precision control. 


\subsubsection{Terminal Sliding Mode Control}

The sliding manifold for this controller is as follows

$$
s=\alpha_{v}+\mathrm{K}_{1} \alpha_{x}+\mathrm{K}_{2} \beta
$$

where $s \in \mathbb{R}^{3 n}, K_{1} \in \mathbb{R}^{3 n \times 3 n}, K_{2} \in \mathbb{R}^{3 n \times 3 n}$, and $\beta \in \mathbb{R}^{3 n}$. It can be expanded into the following form

$$
s_{i}=\alpha_{v i}+\mathrm{K}_{1 i} \alpha_{x i}+\mathrm{K}_{2 i} \beta_{i}\left(\alpha_{x i}\right)
$$

where $\alpha_{x i}$ and $\alpha_{v i}$ are the lumped errors defined in Equation (2.65) and (2.66), $\mathrm{K}_{1 i}$ and $\mathrm{K}_{2 i}$ are positive scalar number, and $\beta_{i}\left(\alpha_{x i}\right)=\left(\beta_{i 1}\left(\alpha_{x i, 1}\right), \cdots, \beta_{i m}\left(\alpha_{x i, 3}\right)\right)^{T} \in \mathbb{R}^{3}$ is expressed as

$$
\begin{aligned}
& \beta_{i j}\left(\alpha_{x i, j}\right) \\
& =\left\{\begin{array}{c}
\alpha_{x i, j}{ }^{p / q} \\
\left(2-\frac{p}{q}\right) \mu^{p / q^{-1}} \alpha_{x i, j}+\left(\frac{p}{q}-1\right) \mu^{p / q^{-2}} \operatorname{sgn}\left(\alpha_{x i, j}\right) \alpha_{x i, j}{ }^{2}
\end{array}\right.
\end{aligned}
$$

The first condition applies when $\bar{s}=\alpha_{v i, j}+\mathrm{K}_{1 i} \alpha_{x i, j}+\mathrm{K}_{2 i} \alpha_{x i, j} p / q=0$ or $\left|\alpha_{x i, j}\right|>\mu$, where $j=$ $1, \cdots, 3$ and $\mu$ is a small positive constant, otherwise, second condition is applied. $\operatorname{sgn}(*)$ is the mathematical sign function, where $p$ and $q$ are two positive odd number and $p<q$.

Lemma 5.1 [55]: if the sliding manifold $s=\bar{s}=0$ is reached, then the absolute state error $e_{x}$ converges to zero in finite time. $\dot{V}$ is strictly negative which implies that the lumped state error $\alpha_{x}$ converges to zero in finite time.

\subsubsection{Control Law Formulation}

An unknown function $f_{i}\left(x_{i}, v_{i}\right)$, where $i=1,2, \cdots, n$, is developed using $\mathrm{CNN}$ approximation to estimate the system dynamic function $F$. 


$$
f_{i}\left(x_{i}, v_{i}\right)=\widehat{W}_{i} t_{i}\left(x_{i}, v_{i}\right)+\mathrm{E}_{i}
$$

where $\widehat{W}_{i}$ is the optimal matrix, and $\widehat{W}_{i} \in R^{3 \times N_{1}}$ with $N_{1}=9 N_{2}+1$ and $N_{2}$ is the order of the Chebyshev polynomial. $t_{i}\left(x_{i}, v_{i}\right) \in R^{N_{1}}$ is a set of Chebyshev polynomial basis function and $E_{i} \in$ $R^{m}$ is the estimated error for the neural network. The structure of the CNN is shown in Figure 5.1.

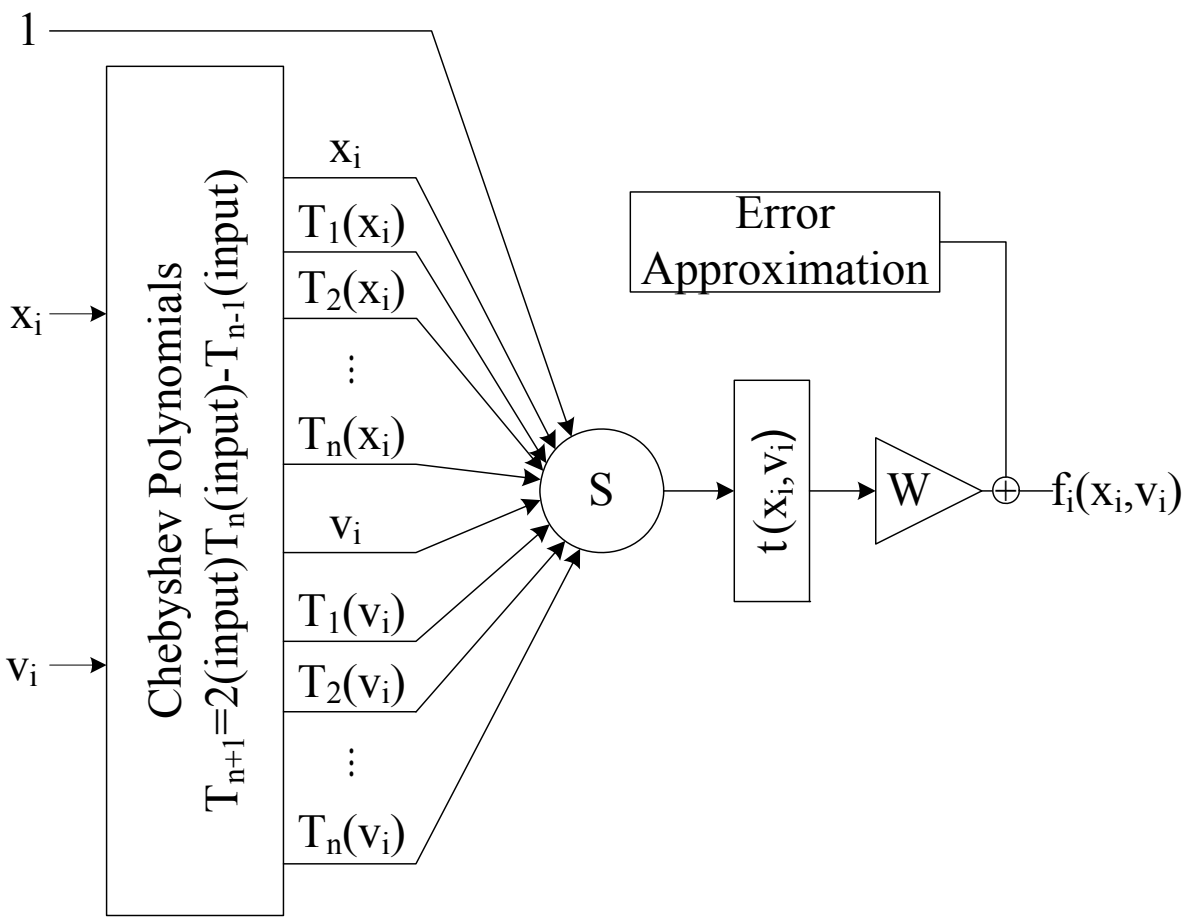

Figure 5.1 CNN structure

$t_{i}\left(X_{i}\right)$ is a set of orthogonal polynomials defined as the solution to the Chebyshev differential equation:

$$
t_{i}\left(X_{i}\right)=\left(1, T_{1}\left(\mathrm{X}_{i, 1}\right), \cdots, T_{N_{2}}\left(\mathrm{X}_{i, 1}\right), \cdots, T_{1}\left(\mathrm{X}_{i, 2 m}\right), \cdots, T_{N_{2}}\left(\mathrm{X}_{i, 2 m}\right)\right)^{T}
$$

These solutions $T_{i}\left(\mathrm{X}_{i, j}\right) i=1,2, \cdots N_{2}$ and $\mathrm{j}=1,2, \cdots 3 \mathrm{~m}$ are obtained by the two-term recursive formula

$$
T_{i+1}\left(\mathrm{X}_{i, j}\right)=2 X_{i, j} T_{i}\left(\mathrm{X}_{i, j}\right)-T_{i-1}\left(\mathrm{X}_{i, j}\right)
$$


and here is the example of the first few Chebyshev polynomials

$$
\begin{aligned}
& T_{0}(a)=1 \\
& T_{1}(a)=a \\
& T_{2}(a)=2 a^{2}-1 \\
& T_{3}(a)=4 a^{3}-3 a
\end{aligned}
$$

In order to complete the stability analysis for this closed-loop system, the following assumptions are specified

Assumption 1: The optimal weight matrix $\widehat{W}_{i}$ is considered to be in a known bounded set $\Omega_{W_{i}}$ where $W_{\min }$ and $W_{\max }$ are the known constants, respectively. [55]

Assumption 2: The $\mathrm{CNN}$ estimated error $\mathrm{E}_{i}$ is considered to be in a known bounded set $\Gamma$ that is defined by where $E_{\min }$ and $E_{\max }$ are the known constants, respectively. [55]

In order to guarantee that the estimated $\mathrm{CNN}$ weight matrices remain within some known bounded sets, the smooth projection algorithm from [55] is used for the CNN weight update. Let the estimated weight matrix for $\widehat{W}_{i}$ be $W_{i}$, and define a smooth projection of $W_{i}$ as

$$
\pi_{i}\left(W_{i}\right)=W_{\pi i}=\pi_{i, j k}\left(W_{i, j k}\right)
$$

where $j=1,2,3$ and $k=1,2, \cdots N_{1}$, and the projection operator $\pi_{i, j k}: R \rightarrow R$ is a real-valued smooth nondecreasing function defined by [55]

$$
\begin{gathered}
\pi_{i, j k}\left(W_{i j, k}\right)=W_{i, j k}, \forall W_{i, j k} \in\left[W_{i \min }, W_{i \max }\right] \\
\pi_{i, j k}\left(\mathrm{~W}_{i, j k}\right) \in\left[W_{i \min }-\varepsilon \omega_{i}, W_{i \max }+\varepsilon \omega_{i}\right], \forall W_{i, j k} \in \mathbb{R}
\end{gathered}
$$

where $\varepsilon \omega_{i}$ is a small positive constant selected by the controller designer.

The control law for the $i$ th follower agent in the multi-agent system is now given by

$$
u_{i}=g_{i}^{-1}\left(-\pi_{i}\left(W_{i}\right) t_{i}-\psi_{i}-\mathrm{K}_{1 i} s_{i}-\mathrm{K}_{2 i} s_{i}{ }^{\phi}\right), \quad i=1,2, \cdots, n
$$


where the robust term $\psi_{i} \in R^{m}$, is used to counteract the external disturbance and CNN approximation error. The same robust controller is used in Equation (4.8). $K_{1 i}$ and $K_{2 i}$ are positive

definite, diagonal, and constant matrices, respectively; and $s_{i}{ }^{\phi}=\left(s_{i 1} \phi, s_{i 2} \phi, \cdots, s_{i m}{ }^{\phi}\right)^{T}$. And the compact from is

$$
u=g^{-1}\left(-\pi(W) t-\psi-\mathrm{K}_{1} s-\mathrm{K}_{2} s^{\phi}\right)
$$

\subsubsection{Stability Analysis}

In the following section, the stability analysis shows that the control law defined by Equation (5.11) for the predefined closed-loop system guarantees semi-global uniformly ultimately boundedness of the relative states of the space vehicles to any desired formation.

Theorem 5.1 [55]: For the multiple spacecraft formation flying mathematical model in Equation (2.48) to (2.49), the sliding manifold is chosen as Equation (5.2) or (5.1), the control law is defined as Equation (5.7), the projection algorithm is given by Equation (5.8) and (5.9) and the adaptive laws are provided by Equation (5.11) and the bounds on the external disturbances on the system is assumed as given by Equation (2.34) to (2.36). For a sufficiently large positive constant $s_{\max }$, if the initial conditions satisfy

$$
s^{T}(0) s(0) \leq \frac{2 s_{\max }}{\lambda_{\max }\left(M_{2}\right)}
$$

$\alpha_{x}(0)$ is bounded, then $s$ is semi-global uniformly ultimately bounded.

Proof: Consider the following Lyapunov function as stated in [55]

$$
V=\frac{1}{2} s^{T} M^{-1} s+\sum_{i=1}^{n} V_{W i}
$$

The time derivative of this Lyapunov function is shown below 


$$
\dot{V}=s^{T} M^{-1} \dot{s}+\sum_{i=1}^{n} \dot{V}_{W i}
$$

which contains two independent terms.

To begin the proof, consider the first term in Equation (5.14). Where the time derivative of the sliding manifold from Equation (5.1) is shown below

$$
\dot{s}=\dot{\alpha}_{v}+\mathrm{K}_{1} \dot{\alpha}_{x}+\mathrm{K}_{2} \dot{\beta}=\dot{\alpha}_{v}+\mathrm{K}_{1} \alpha_{v}+\mathrm{K}_{2} \dot{\beta}
$$

By multiplying $M^{-1}$ to both side of Equation (5.15), we have

$$
M^{-1} \dot{s}=M^{-1} \dot{\alpha}_{v}+M^{-1} \mathrm{~K}_{1} \alpha_{v}+M^{-1} \mathrm{~K}_{2} \dot{\beta}
$$

Then by substituting the first term on the right-hand side of Equation (5.16) with the corresponding parameters from Equation (2.68), the term $M^{-1} \dot{s}$ can be expressed as follows

$$
M^{-1} \dot{s}=\mathrm{F}+g \mathrm{u}+\vartheta-X_{d}+M^{-1}\left(\mathrm{~K}_{1} \alpha_{v}+\mathrm{K}_{2} \dot{\beta}\right)
$$

The control law stated in Equation (5.11) is substituted into Equation (5.17) to obtain

$$
M^{-1} \dot{s}=\pi(\widetilde{W}) t-\psi-K_{1} s-K_{2} s^{\phi}+\vartheta+E-X_{d}+\zeta
$$

where $\zeta=M^{-1}\left(\mathrm{~K}_{1} \alpha_{v}+\mathrm{K}_{2} \dot{\beta}\right)$, and $\widehat{W}=\operatorname{diag}\left\{\widehat{W}_{1}, \widehat{W}_{2}, \cdots \widehat{W}_{n}\right\}, \pi(\widetilde{W})=\widehat{W}-\pi(W), \pi(W)=$ $\operatorname{diag}\left\{\pi\left(W_{1}\right), \pi\left(W_{2}\right), \cdots \pi\left(W_{n}\right)\right\}, \quad t=\left(t_{1}{ }^{T}, t_{2}{ }^{T}, \cdots t_{n}{ }^{T}\right)^{T}, \quad K_{1}=\operatorname{diag}\left\{K_{11}, K_{12}, \cdots, K_{1 n}\right\}, \quad K_{2}=$ $\operatorname{diag}\left\{K_{21}, K_{22}, \cdots, K_{2 n}\right\}, \psi=\left(\psi_{1}{ }^{T}, \psi_{2}{ }^{T}, \cdots \psi_{n}{ }^{T}\right)^{T}$, and $E=\left(E_{1}{ }^{T}, E_{2}{ }^{T}, \cdots E_{n}{ }^{T}\right)^{T}$.

Consider the second term in Equation (5.13), the time derivative of the adaptive law for $W_{i}(i=1,2, \cdots, n)$ is

$$
\dot{W}_{i}=\delta_{i} s_{i} t_{i}^{T}
$$


where $\delta_{i}$ is a positive number. A Lyapunov function for this adaptive law is defined in [55] where $V_{w i}(i=1,2, \cdots, n)$ is positive definite with respect to $\widetilde{W}_{i, j k}$ for $\widehat{W}_{i, j k} \in\left[W_{i \min }, W_{i \max }\right]$. Furthermore, the time derivative of $V_{W i}(i=1,2, \cdots, n)$ is

$$
\dot{V}_{W i}=-\frac{1}{\delta_{i}} \sum_{j=1}^{m} \sum_{k=1}^{N_{1}} \widetilde{W}_{\pi i, j k} \dot{W}_{i, j k}
$$

Substituting Equations (5.20) and (5.18) to Equation (5.14), and applying the inequalities and the simplification proposed in [55], the time derivative of the Lyapunov function, yields

$$
\dot{V} \leq-\left(\lambda_{\min }\left(\mathrm{K}_{1}\right)-c\right) s^{T} s+\frac{\zeta_{M}^{2}}{4 c}+n \epsilon
$$

where $c$ is a positive constant satisfying $c<\lambda_{\min }\left(\mathrm{K}_{1}\right)$ and $\lambda_{\min }(*)$ denotes the minimum eigenvalue of a matrix. $\zeta_{M}$ is a sufficiently large constant. Thus, $\dot{V}$ is strictly negative when $s$ is outside of the following set

$$
\left\{s:\|s\| \leq \sqrt{\frac{\frac{\zeta_{M}^{2}}{4 c}+n \epsilon}{\lambda_{\min }\left(\mathrm{K}_{1}\right)-c}}\right\}
$$

and as stated in the initial condition, for a sufficiently large positive constant $s_{\text {max }}$, the following compact set can be constructed

$$
\Omega_{s}=\left\{s \mid s^{T} s \leq \frac{2 s_{\max }}{\lambda_{\max }\left(M_{2}\right)}\right\}
$$

where $\lambda_{\max }(*)$ denotes the maximum eigenvalue of a matrix, and $M_{2}=M^{-1}$. Because the sets $\Omega_{d}$, defined in Equation (2.51) and $\Omega_{s}$ are compact in $R^{3 m}$ and $R^{m n}$, respectively. The variable $\zeta$ 
has a maximum $\zeta_{M}$ on the compact set $\Omega_{d} \times \Omega_{s}$, which implies that $\|s\|$ decreases whenever $s$ is outside the compact set, and therefore, it is concluded that $s$ is uniformly ultimately bounded.

\subsection{RESUlTS AND DisCUSSIONS}

The control law developed in this chapter is the combination of adaptive control, robust control, and Neural Network control. The ability of robust control has been demonstrated in the previous chapter. Even though a large range of external disturbances can be handled by the robust control developed in Chapter 4, it only works in a bounded interval. However, adaptive control is capable of handling unknown parameters. The disadvantages from the most commonly used adaptive control in literature are high-gain feedback, and the linearity in unknown parameters. Therefore, the proposed control law has combined Neural Network and adaptive control to regulate the system in more difficult situations. Chebyshev Neural Network is implemented, and this allows the control system to learn the state of the plant. CNN estimates the unknown dynamics in the system with the defined polynomial functions, Equation (5.5) and (5.6). These equations allowed $\mathrm{CNN}$ to learn the behaviour of the plant on-line during the mission. That makes the control law proposed does not require the knowledge of the dynamic system.

Numerical results are provided to demonstrate the effectiveness of the proposed controller. Similar to the previous chapters, there are 6 agents in the consensus network. The nonlinear equation of motion, and the time varying external disturbances use in this chapter are the same as those described in Chapter 2. The same two initial state errors are examined for comparison with the results calculated in Chapter 3 and Chapter 4. The weighted adjacency matrices and the formation configuration maintain the same value as defined in Chapter 3. Since the control law developed in this chapter is different than the one used in Chapter 3 and Chapter 4, the control parameters will be different.

Following the derivation of the control law in Chapter 5.1.2, the defined control parameters used in this simulation are, $\emptyset=3 / 5, K_{1}=1 \times 10^{-2}, K_{2}=1 \times 10^{-2}$ for the TSMC in Equation (5.1); The parameters for robust control in Equation (4.8) are, $\kappa_{i}=1 \times 10^{-1}, \epsilon=0.01$; The order of the CNN used is 3, and the parameter in Equation (5.19) is $\delta_{i}=10$; By trial and error, the control gains used in (5.11) are $K_{1 i}=\left[1 \times 10^{-2} \quad 1 \times 10^{-2} \quad 1 \times 10^{-2} 1 \times 10^{-2}\right.$ $\left.1 \times 10^{-2} 1 \times 10^{-2}\right], \quad$ and $K_{2 i}=\left[\begin{array}{lllll}1 \times 10^{-1} & 1 \times 10^{-1} & 1 \times 10^{-1} & 1 \times 10^{-1} & 1 \times 10^{-1}\end{array}\right.$ 
$1 \times 10^{-1}$ ], where $i=1,2, \cdots, 6$. Since the order of the Chebyshev polynomials used in this chapter is 3 , therefore the initial weight matrix of the $\mathrm{CNN}$ is a 3 by 13 zeros matrix.

It is expected that the new control law may take less control effort than the previous robust SMC. However, the effectiveness of the CNN adaptive control depends on its estimate ability. There are possibilities that the on-line estimated model becomes uncontrollable. A robust term is added in order to make the controller more robust when estimation errors occur.

\subsubsection{Mission Scenarios}

There are 6 agents in this consensus tracking system and the two sets of initial state errors used to obtain the following results are defined in Chapter 3.2.1.

The same external disturbances, sensor faults, actuator faults and spacecraft' masses considered in previous mission and system requirement, Chapter 4.2.1, are applied in this mission. In addition, reference trajectories are changed from the predefined orbit, to doubling its speed during the mission. Furthermore, the formation of the spacecraft will be reconfigured during the mission. Since there are 6 agents and this configuration has been previously investigated, see Figure 3.2, a regular hexagon is used as the reference formation in this chapter. The distance between each consecutive agent is $0.5 \mathrm{~km}$, and each agent is 60 degrees apart from one another; measured from the centre of the formation. The advantage of applying adaptive control is changing system parameters without adjusting control parameters manually. Therefore, one of the mission requirements is to reconfigure the formation of the agents. Moreover, the formation changes from planar to three dimensions, to help observe the asteroid from different perspectives. The agents are required to orbit around the astronomical body in a regular hexagonal shape for one orbit, then smoothly maneuver into a pyramid formation, see Figure 5.2. The base of the pyramid is placed in the $y-z$ plane. The first 4 agents are located at each corner and form a square base with a side length of $0.5 \mathrm{~km}$. Agent 6 is put at the top of the pyramid which is $1 \mathrm{~km}$ above the centre of the base. Agent 5 is located half way between the base and the tip of the pyramid. 


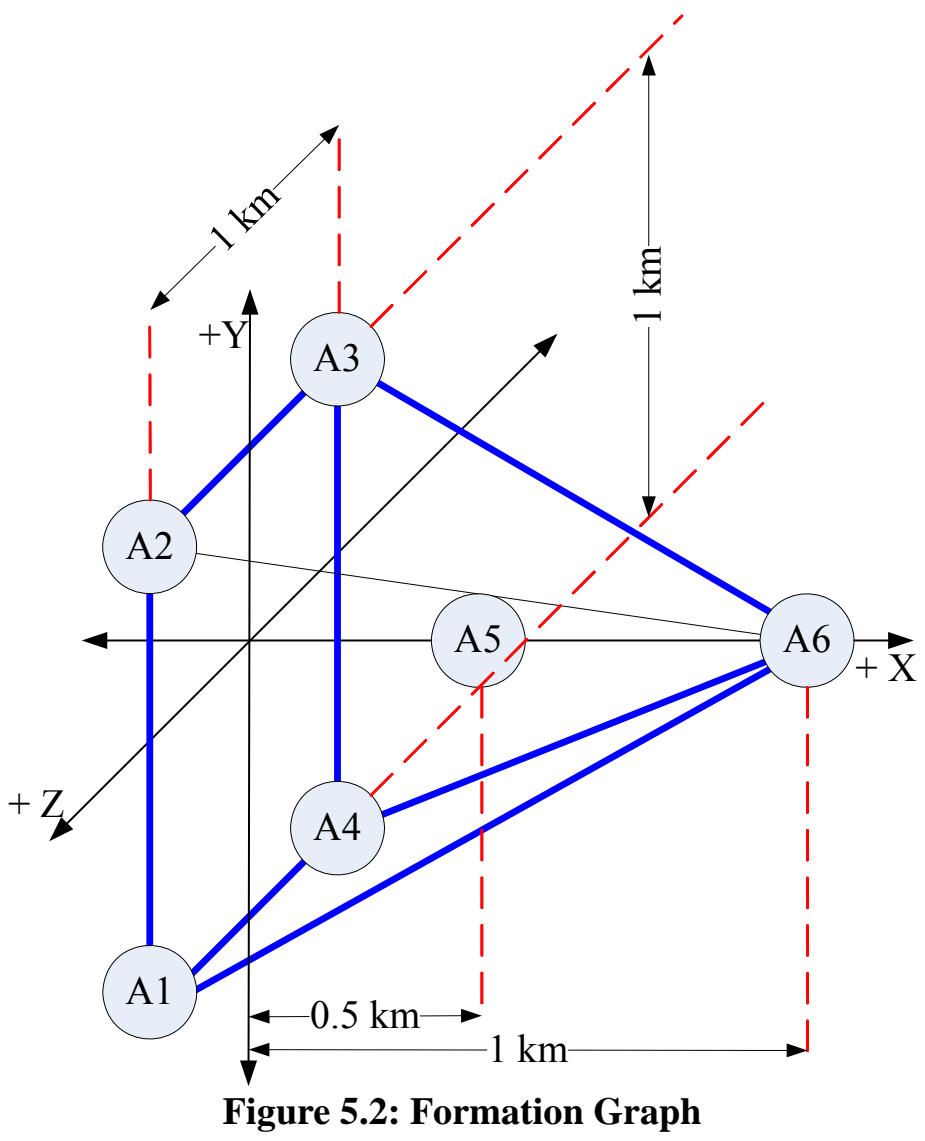

Following are different scenarios where the control law developed above is being examined. A small investigation of the new Terminal Sliding Mode Control (TSMC) with the companion of Chebyshev Neural Network (CNN) is given in Chapter 5.2.2. The system response for large initial error, the results for sensor faults and actuator faults from Chapter 3 and Chapter 4 are brought back in Chapter 5.2.3 to compare the difference between the new and old control law. Chapter 5.2.4 shows that the response remains unaffected with a large unexpected initial mass. Lastly, it is illustrated in Chapter 5.2.5, the reference trajectory and the configuration of the formation are changed during the mission without any adjustment in control parameters.

\subsubsection{Effect of Using CNN to Estimate an Unknown System}

The dynamic system, the desired trajectory and the disturbance model used in this chapter are the same as those defined in Chapter 2. Therefore, the desired system control input with and without disturbance is the same as in Figure 2.7 and Figure 2.8, respectively. We will start with studying the performance of a single spacecraft applying the new developed control law. The proposed control law suggests that there is no need for the control system to know the dynamic 
model. The estimated system uses Chebyshev equations to assess the dynamic model. There is a learning process to project a proper state of the system, and the comparison of the estimated and true value of the dynamics are given in Figure 5.3 and Figure 5.4. The same as with all learning process, knowledge advanced from a mistake. The Chebyshev algorithm learns from the previous estimated state error and projects the future state value. Robust control is included in the control law, even without the estimated state value, the control law attempts to reduce the state error. However, with the combination of $\mathrm{CNN}$ and robust control, it is an advantage that there is an option where the estimation system does not learn when the estimated error is relatively large. It is illustrated in both Figure 5.3 and Figure 5.4, however, it is clearer when a disturbance is applied to the system. As shown for the y estimated values in Figure 5.4, it is said that the CNN will not learn when the absolute estimated value is more than 0.003 . Therefore, the line is discontinuous from 0 to 0.2 of an orbit. The estimated state value is dependent on the state error. When the state error is the lower, the currently used Chebyshev polynomial model cannot approximate the exact value, especially to such a small magnitude $10^{-4}$. Consequently, chattering occurs when the estimated error is low.

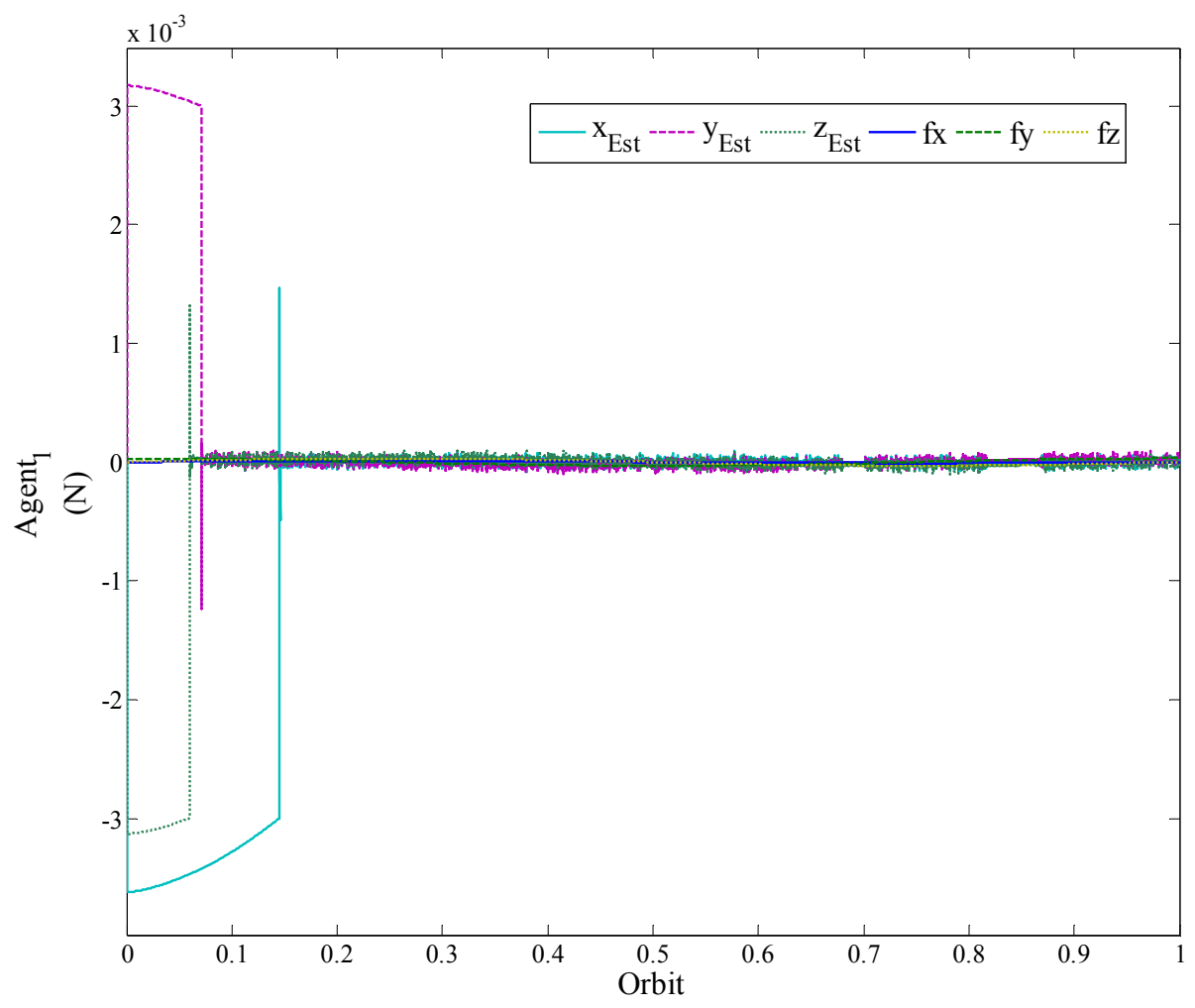

Figure 5.3: Estimation by CNN and $f(x, y, z)=F+\theta-X_{d}$ (without disturbance) 


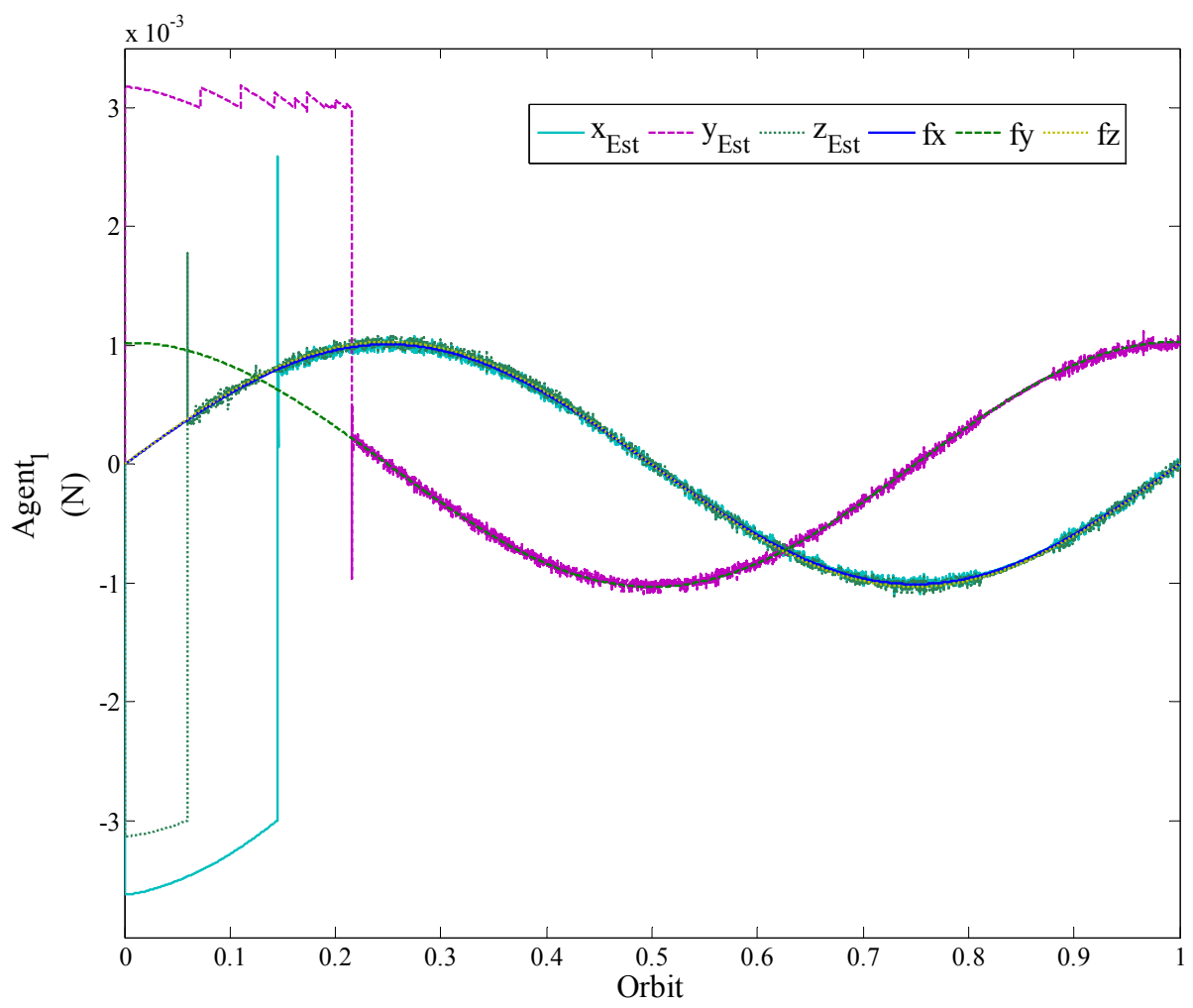

Figure 5.4: Estimation by CNN and $f(x, y, z)=F+\theta-X_{d}$ (with disturbance)
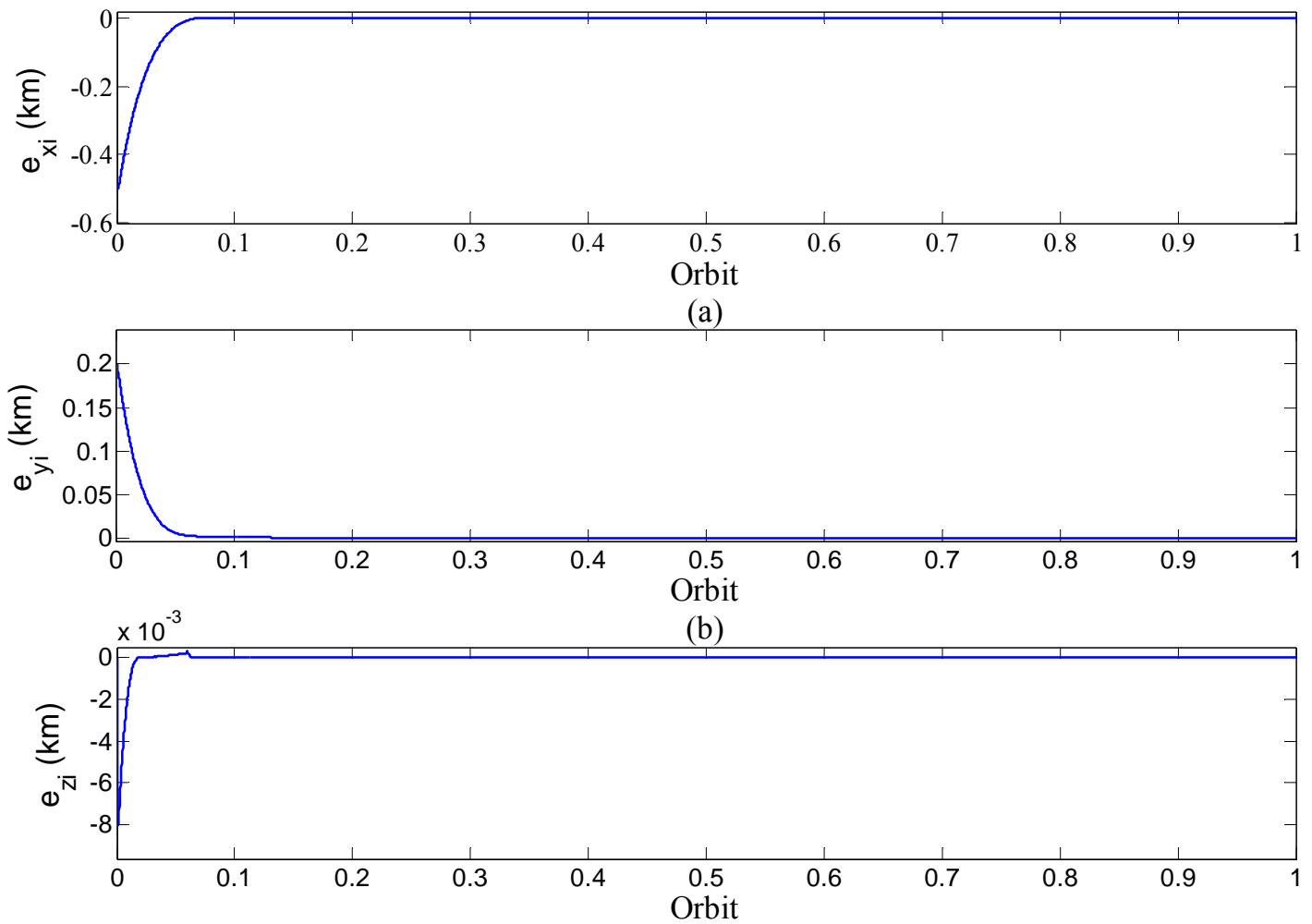

(c)

Figure 5.5: Tracking errors (with disturbance) 
The tracking error where disturbance is applied to the system is shown in Figure 5.5. The results where a disturbance is not applied is very similar. For both scenarios, the error converges to a very small number that is close to zero, and the settling time is about the same. The chattering in the estimated error leads to the chattering in the system control input as shown in Figure 5.6 and Figure 5.7. Besides the chattering, the system control input is comparable to the desired system control input in Chapter 2.
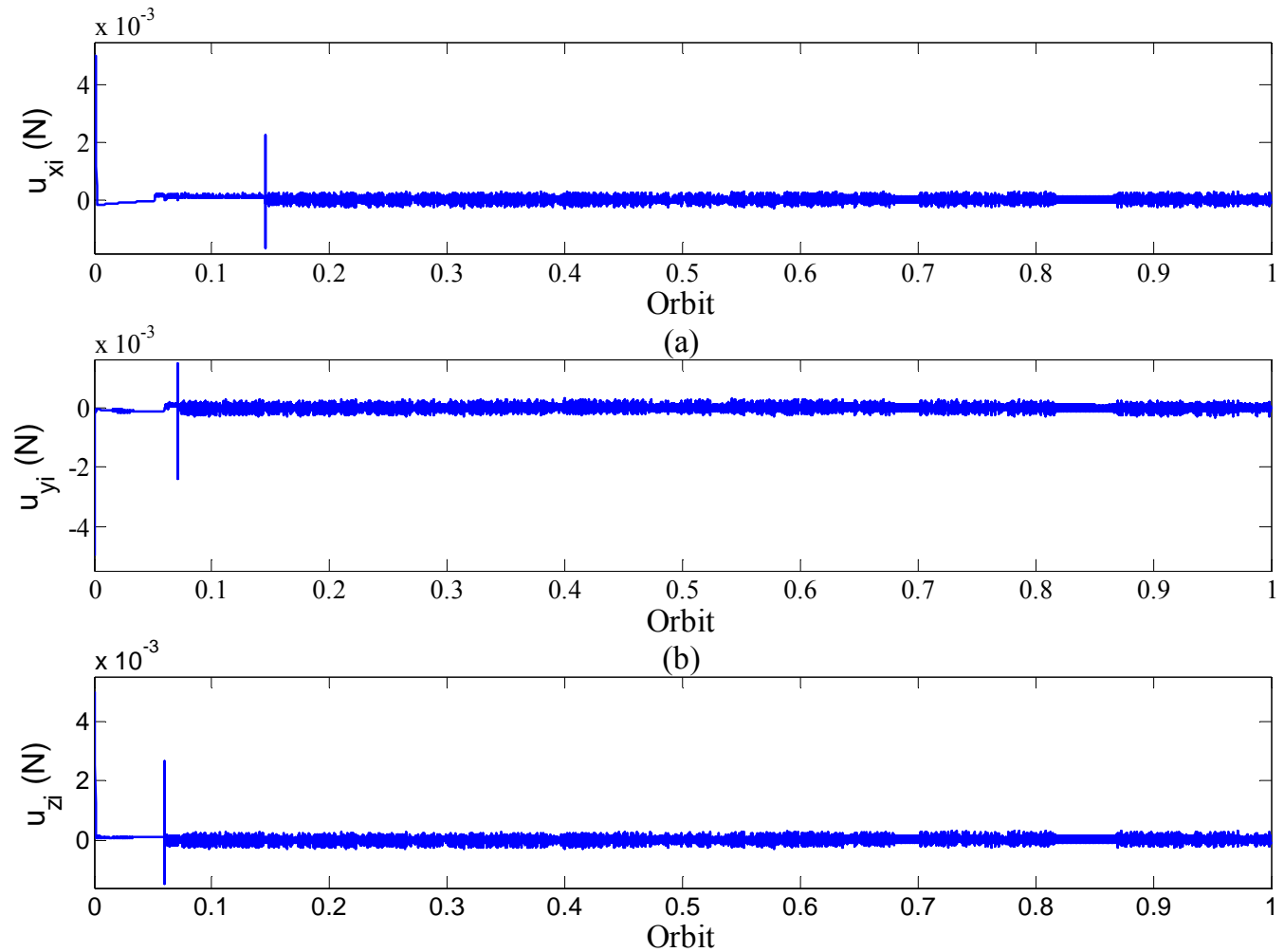

(c)

Figure 5.6: Control input (without disturbance)

After studying the behaviour of a single agent using the new adaptive control law, a centralized and decentralized formation algorithm is then studied. Centralized algorithm is defined as every agent obtains information from the other agents through a virtual leader agent. Decentralized algorithm only allow limited information being transfer using consensus algorithm with the predefined adjacency matrix. 

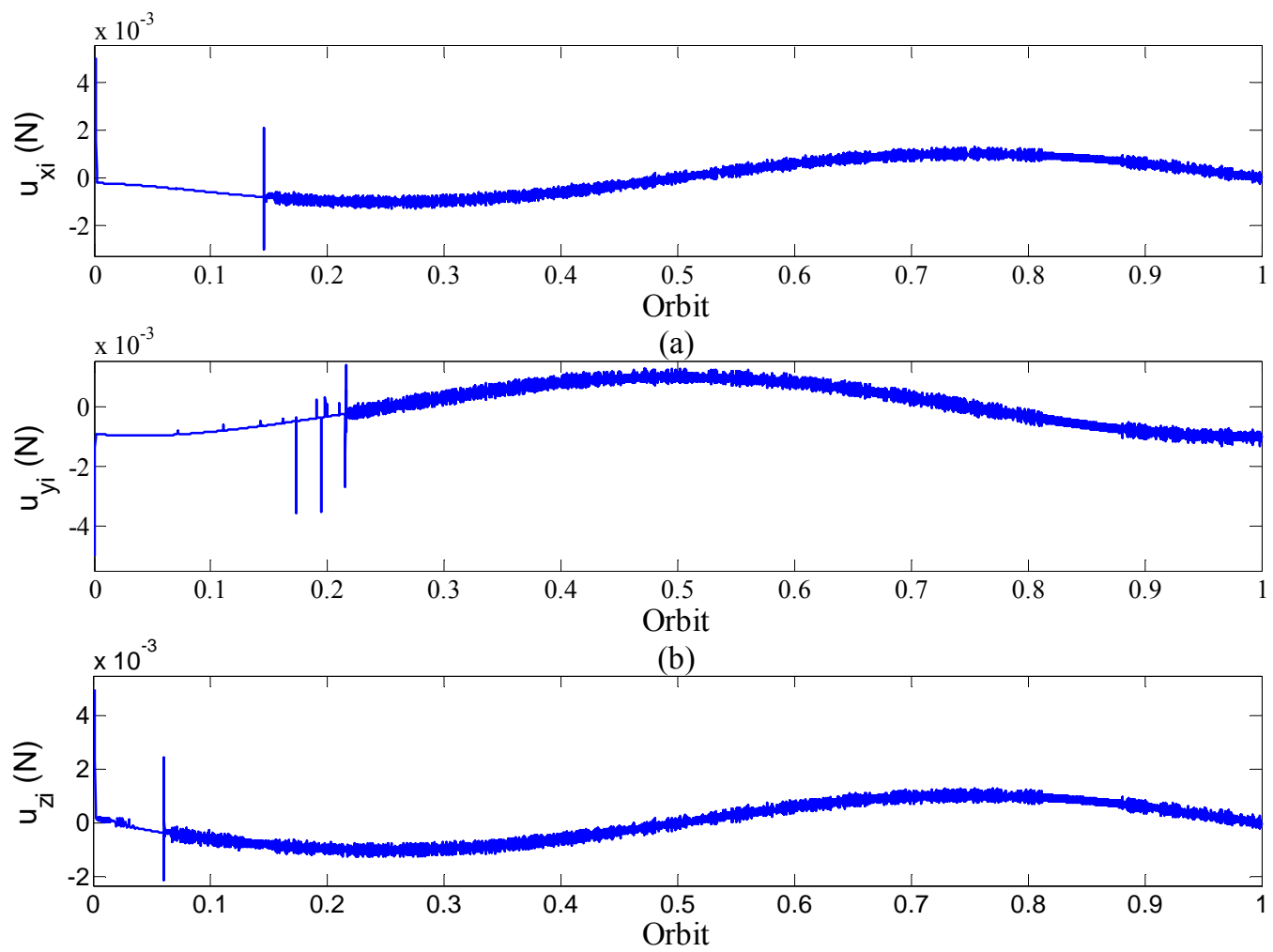

(c)

Figure 5.7: Contol input (with disturbance)

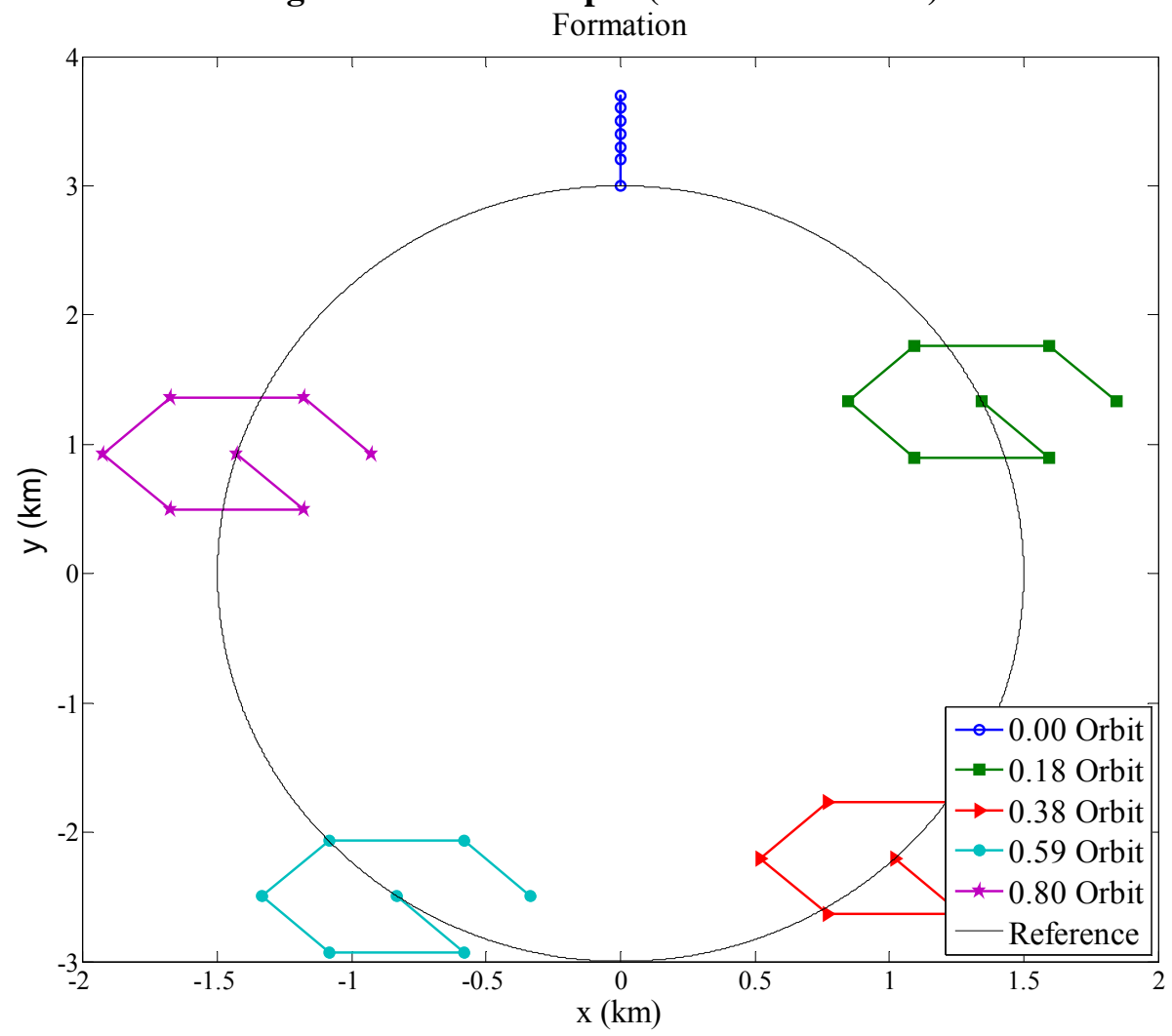

Figure 5.8: System state snap shot at different time, centralized system 
The responses of the state for the centralized and decentralized systems are very similar, and it is shown in Figure 5.8 and Figure 5.9. It is expected that the tracking error is less for the centralized system since each agent is following the position of the leader. However, the relative formation error is less for the decentralized system because the consensus algorithm has taken the relative error into account when controlling the system. Both can be verified through Figure 5.10 and Figure 5.11.
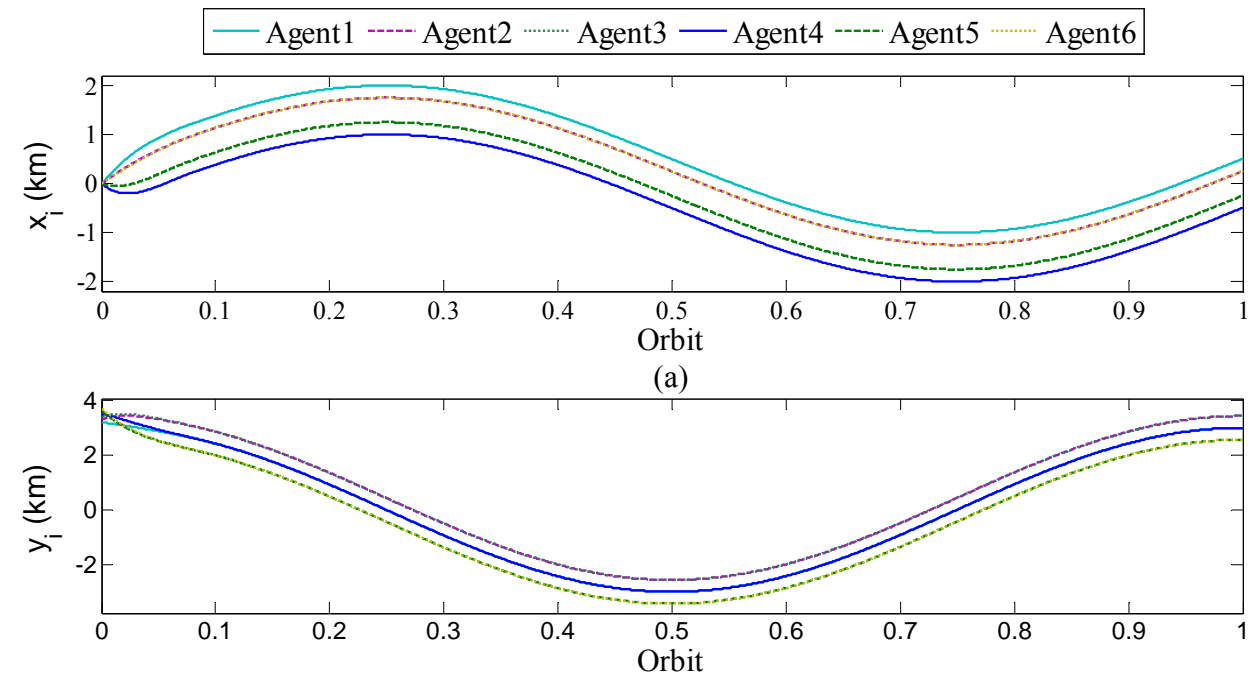

(b)

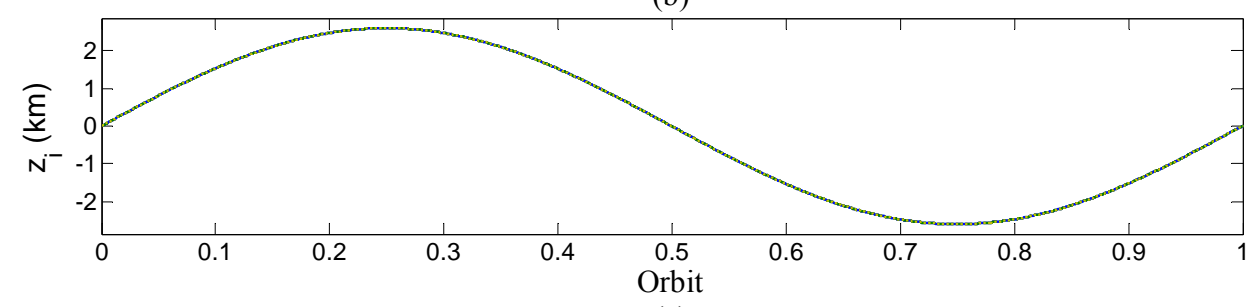

(c)

Figure 5.9: State of all agents, decentralized system 


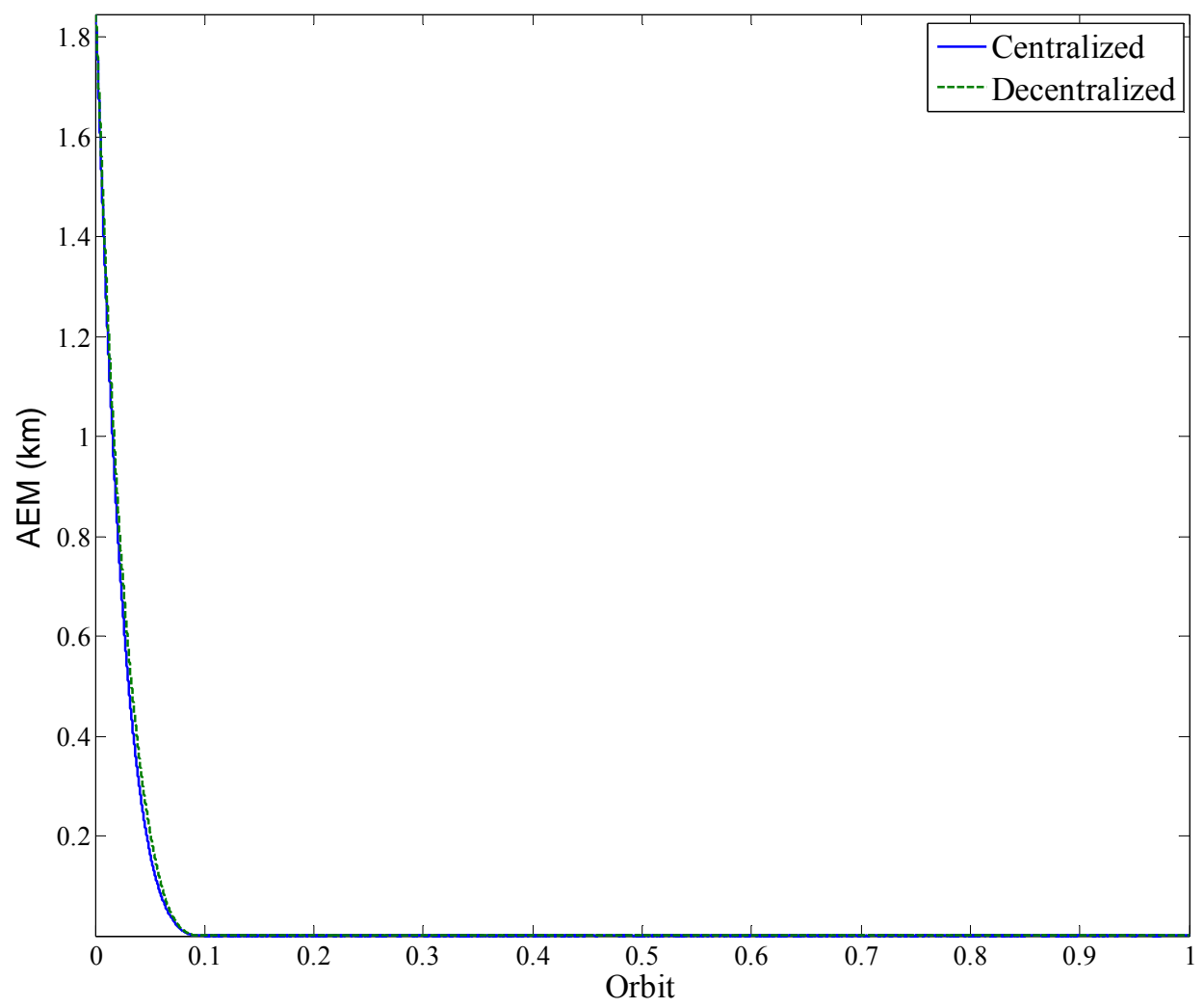

Figure 5.10: AEM, centralized vs. decentralized

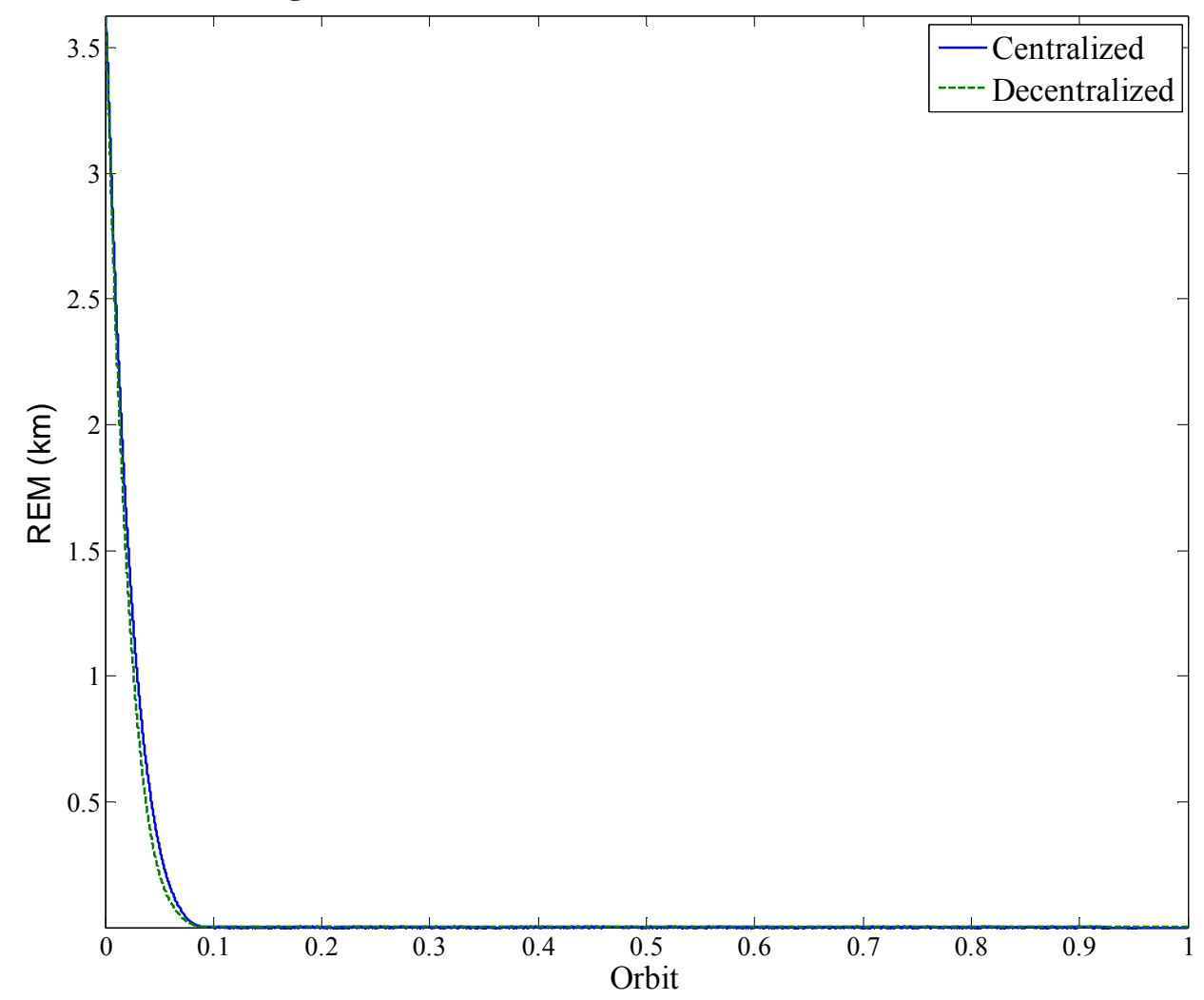

Figure 5.11: REM, centralized vs. decentralized 
A better approach to study the overall control effort for multi-agent is to recognize them as a single system. The overall control effort metric (OCEM) can be calculate as

$$
\text { OCEM }=\sqrt{\sum_{i=1}^{n}\left\|u_{i}\right\|^{2}}
$$

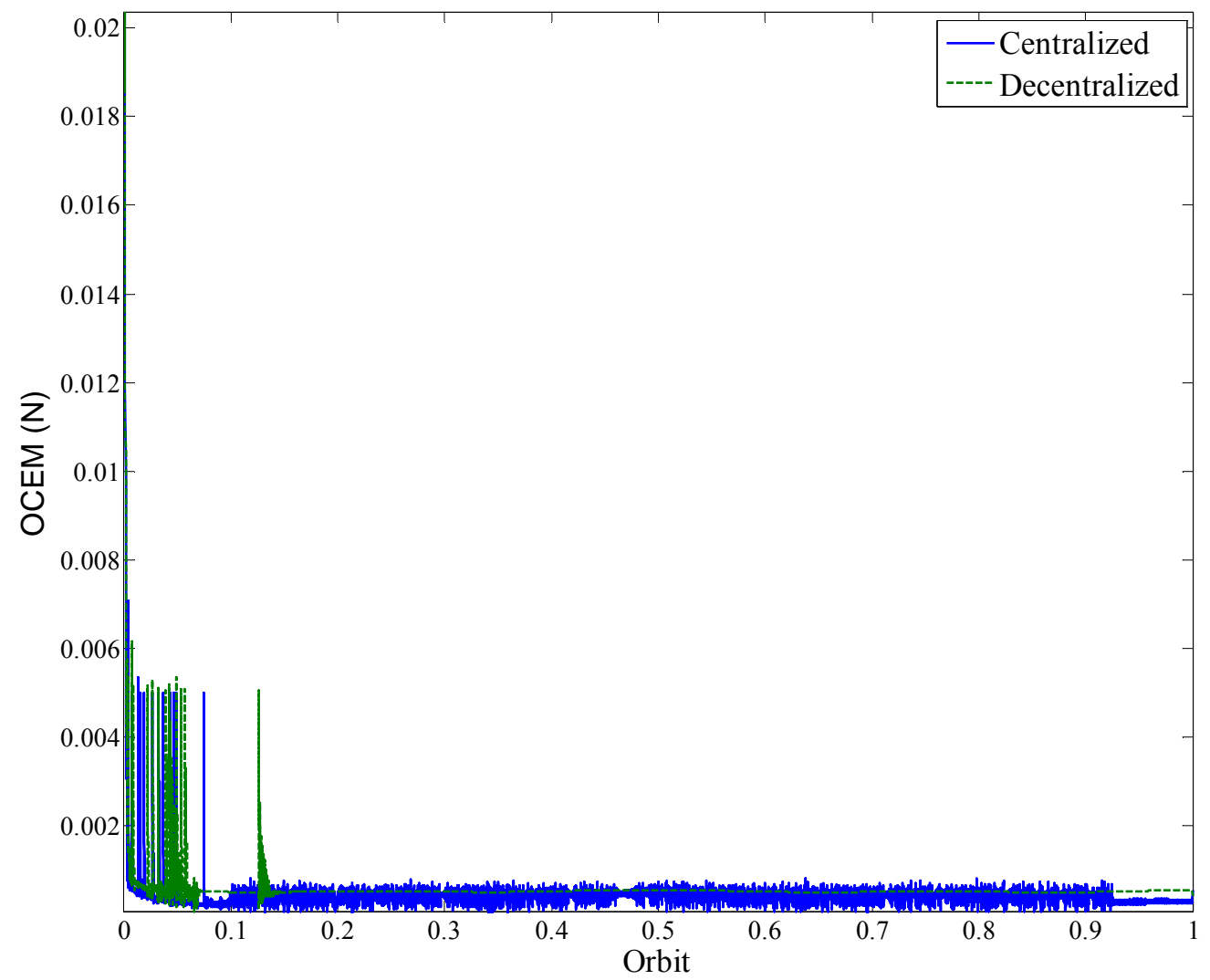

Figure 5.12: OCEM, centralized vs. decentralized 

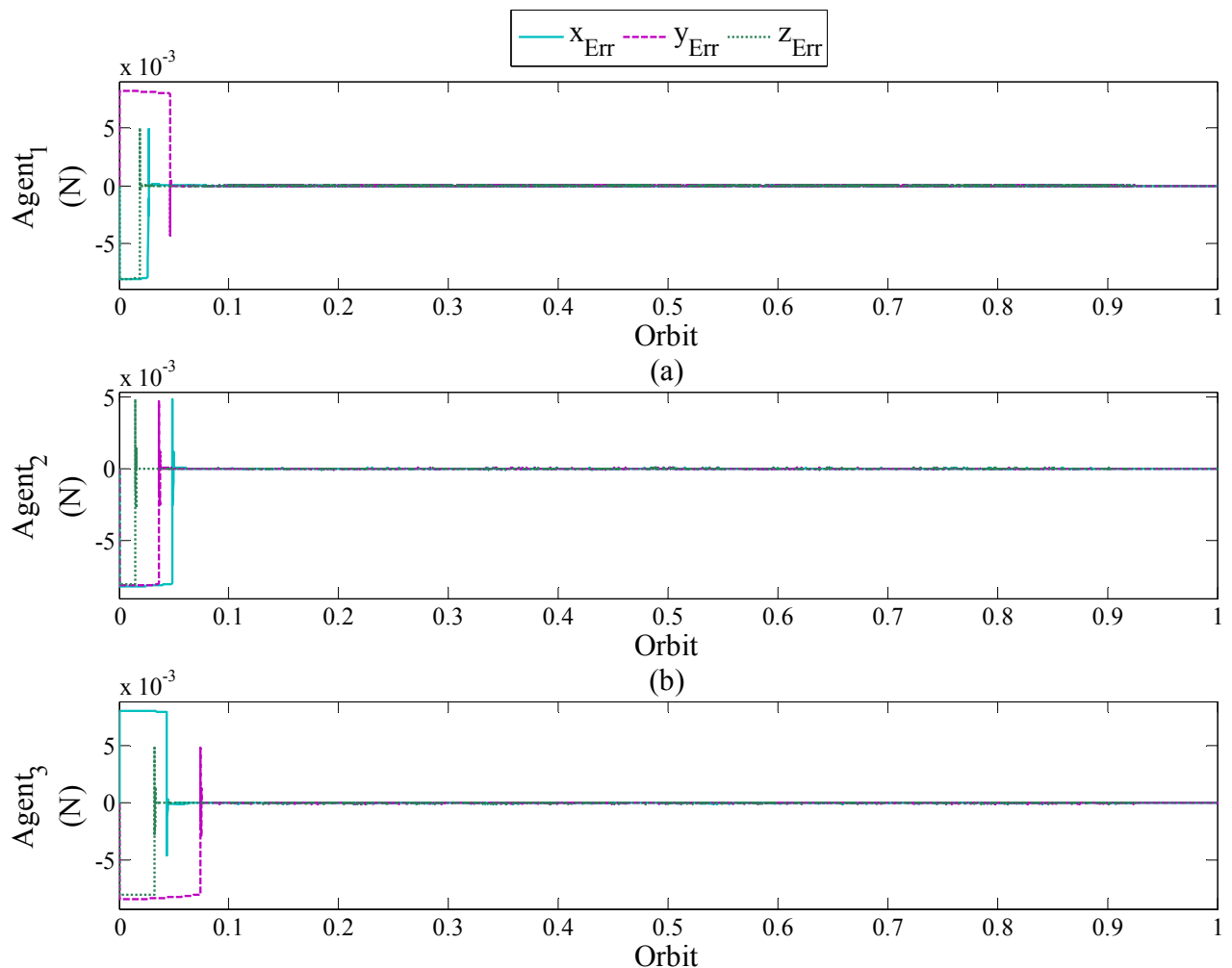

(c)
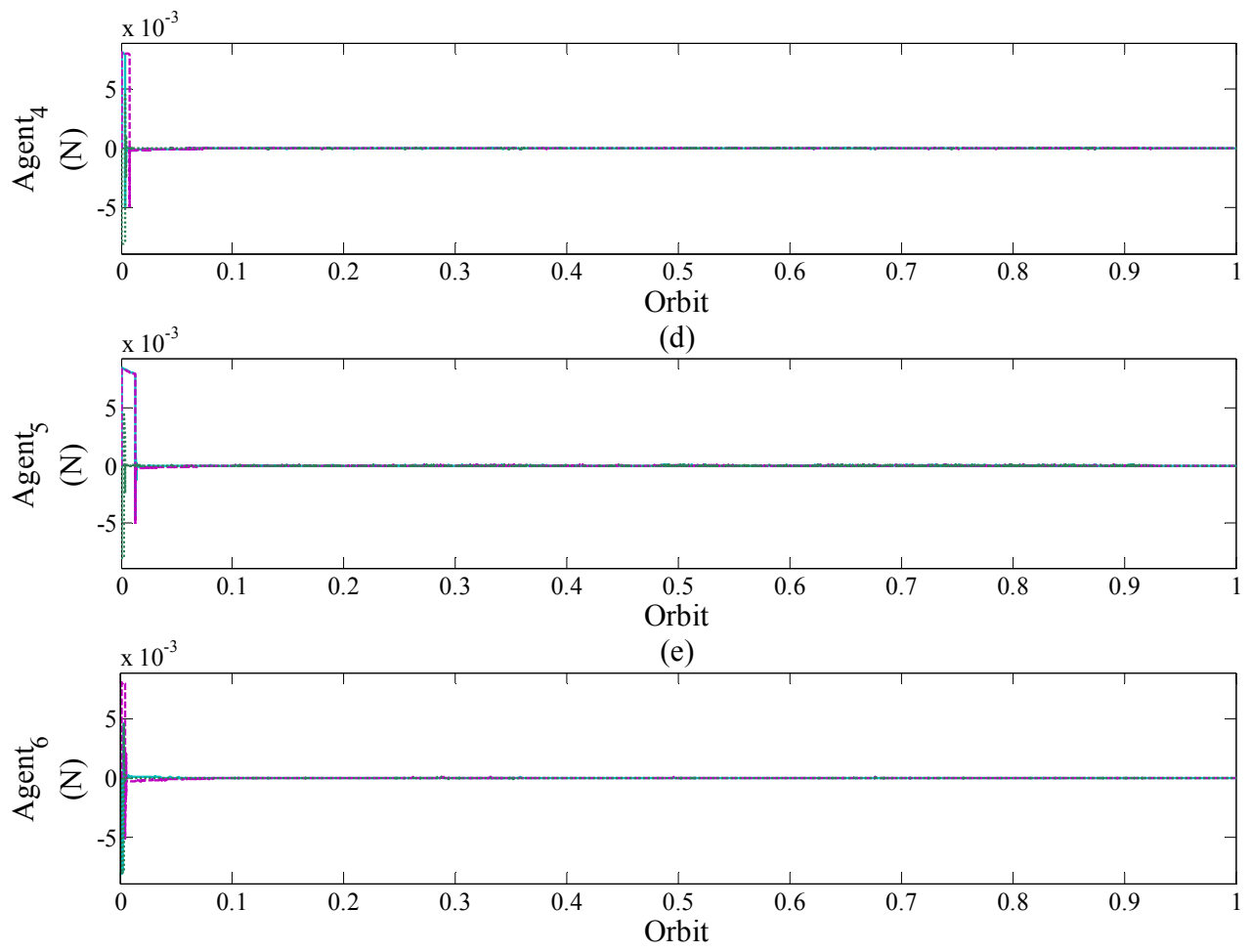

(f)

Figure 5.13: Estimated errors, centralized 

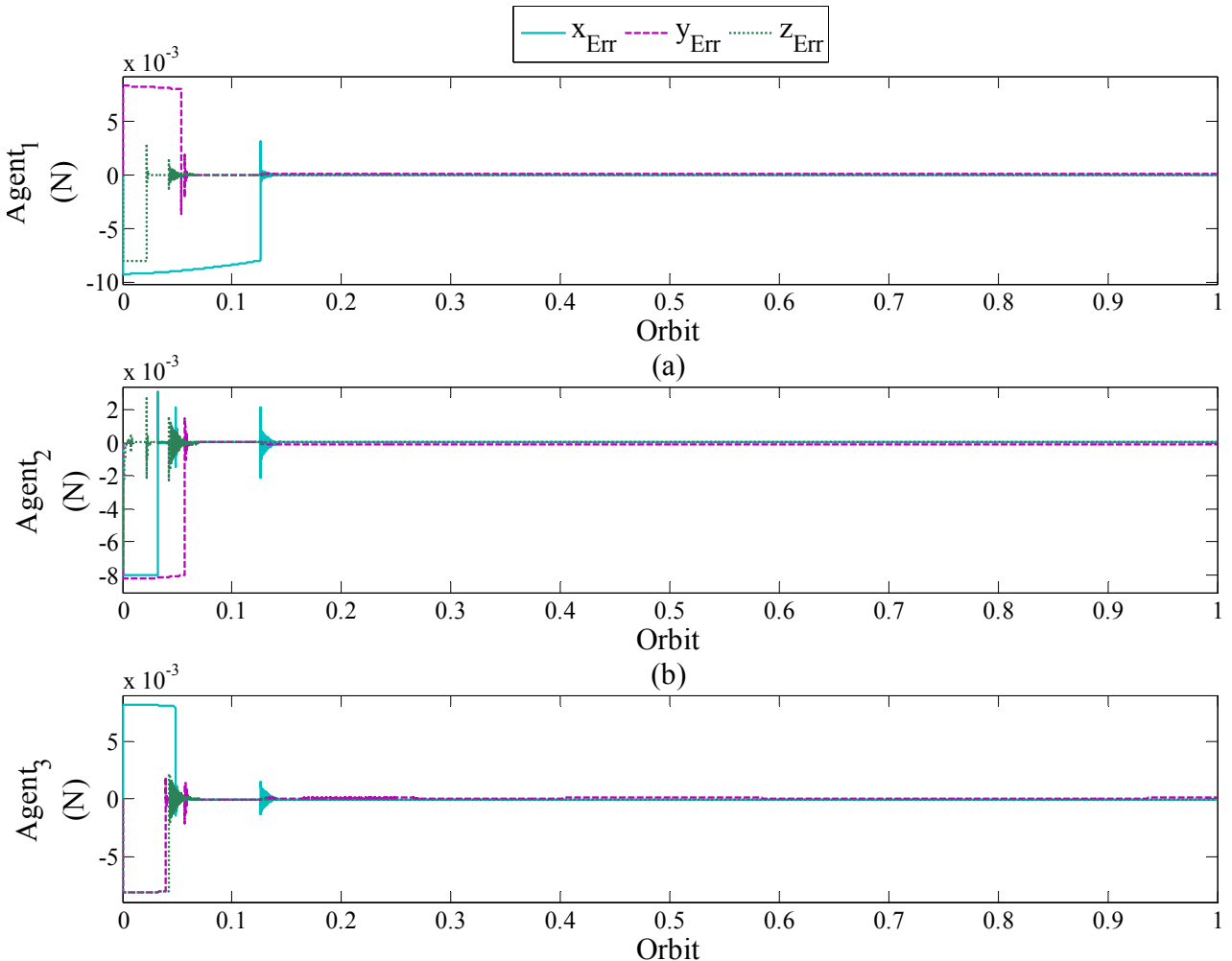

(c)
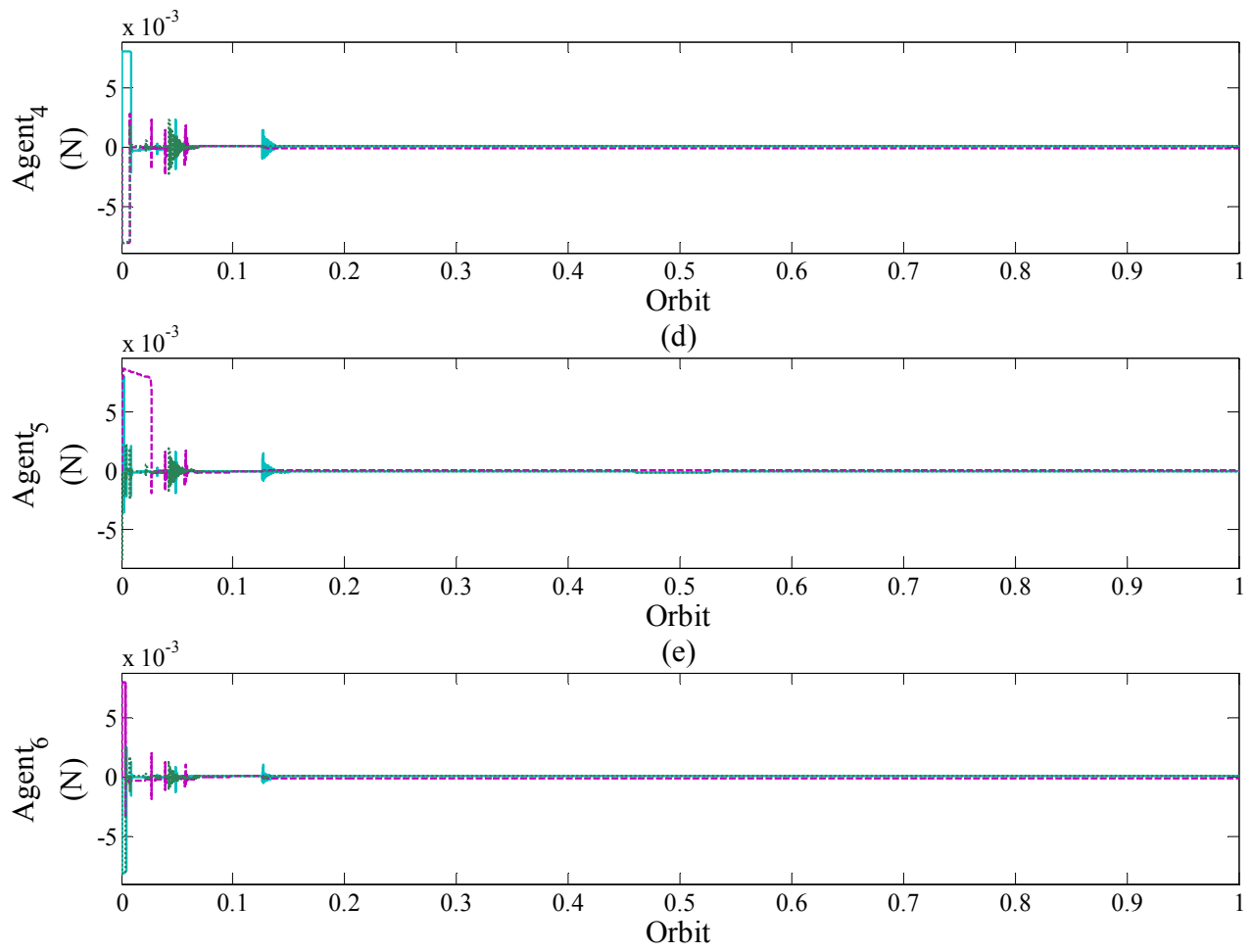

(f)

Figure 5.14: Estimated errors, decentralized 
In Figure 5.12, there is more chattering for the centralized system, however, if chattering is ignored, the value of the overall control effort is lower for centralized system as compared with decentralized formation. Figure 5.13 and Figure 5.14 are the errors between the estimated state values with the true stated values. Figure 5.13 is the estimated error for the centralized system, and Figure 5.14 is the estimated error for the decentralized system. When the formation is centralized, the state error in one agent does not affect the state value of the other agents, therefore, the learning process for the $\mathrm{CNN}$ algorithm is much faster and more stable than the decentralized system.

Throughout the study of a single spacecraft orbiting around an asteroid using the CNN adaptive and robust control, it is verified that besides the unstable estimated error due to the numerical model, the control law is indeed able to control the plant. In addition, the results from the system response and system control input are as good as to the SMC and Robust SMC control.

\subsubsection{Performance Comparison with SMC and Robust Control}

In Chapter 3 the control law developed using SMC is able to control the multi-agent system when large initial state error is applied. However, when the disturbance model is applied at the same time with the $5 \mathrm{mN}$ thruster saturation, the control system failed to force the agents to follow the desired trajectory. The results shown in Chapter 4 have proven that the controller developed in that chapter is more robust. There are improvements in settling time and system control input. In the robust control it is able to control actuator faults that were examined, however, when a lower maximum actuator force is applied, the control system fails to provide good results. Due to the imperfect response from the previous developed control law, these most difficult conditions are once again examined with this newly developed control law.

\subsubsection{Effect of Large Initial State Error with Disturbance}

The adaptive robust control law has been examined when only the large initial state error is applied, the tracking error of the system converges to zero. However, due to the system's lack of information of the system dynamic model, the state error grew faster at the beginning when the CNN adaptive estimate system is in its learning phase. Once the estimate system gathers enough information, the state error is reduced quickly and the state errors converge to a very small value that is close to zero in 1 orbit as the mission required. 
The advantage of an adaptive control, particularly with the use of the proposed neural network model, the system is not sensitive to the external disturbance. The system might take a different learning path for different scenarios.

Figure 5.15 is the tracking error where both harsh environment, thruster saturation, and disturbance model are all included in the plant. Comparing with Figure 3.15, the disturbance has not yet been applied, and the error diverged due to the thruster saturation. Figure 4.6 and Figure 4.7 are the AEM and REM for the robust SMC in Chapter 4, showing that both the tracking and formation have failed due to the lack of sufficient control force. The state error accumulated overtime is the main reason that leads to mission failure. Figure 5.16 is the control input from the new adaptive robust control law. The system has reached saturation due to the large initial state error, however, the adaptive estimate system allows the control system to adjust and adapt to the unpleasant situation. After 0.6 of an orbit, all system control input have reached the minimum control input.
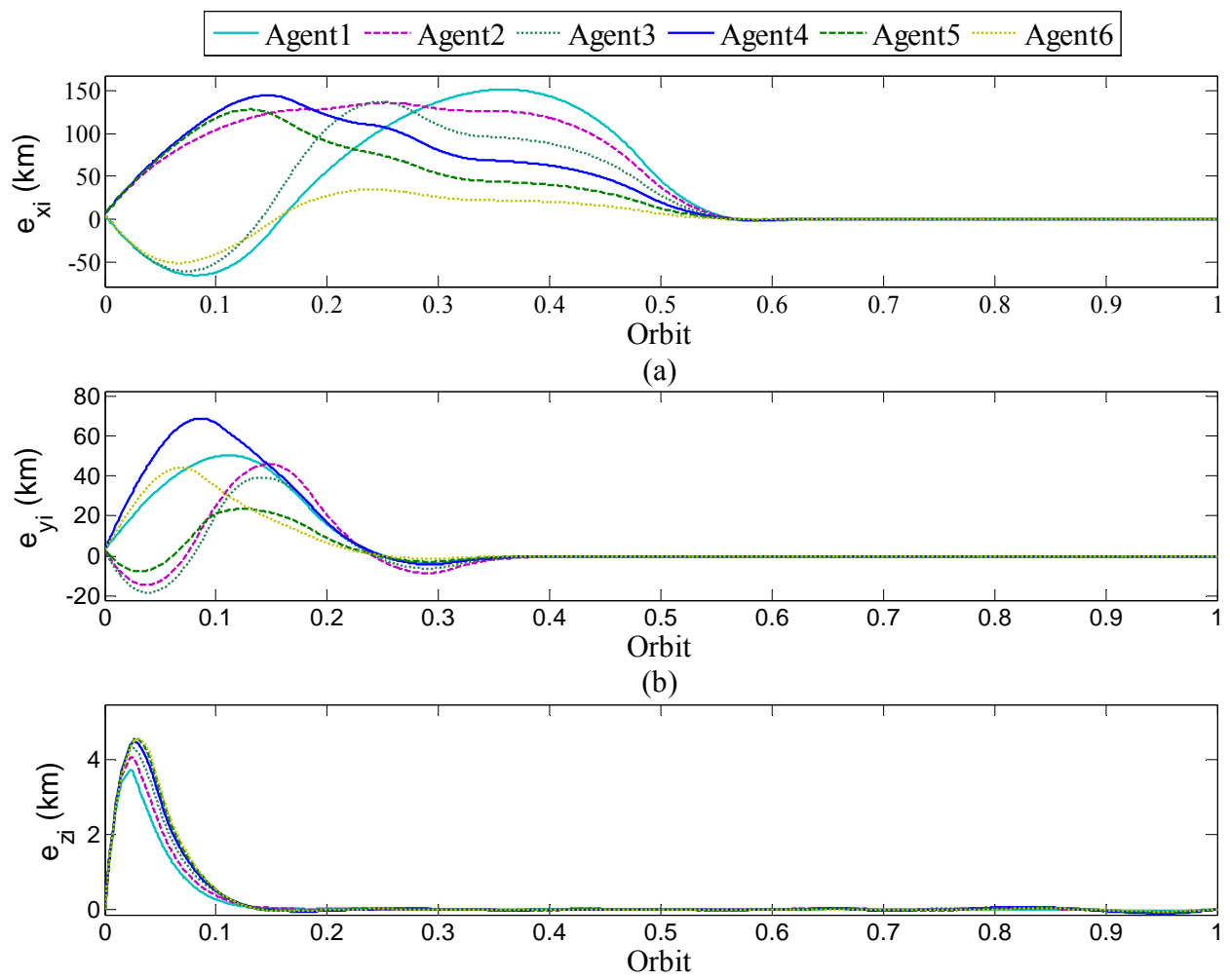

(c)

Figure 5.15: Tracking errors (with disturbance) 

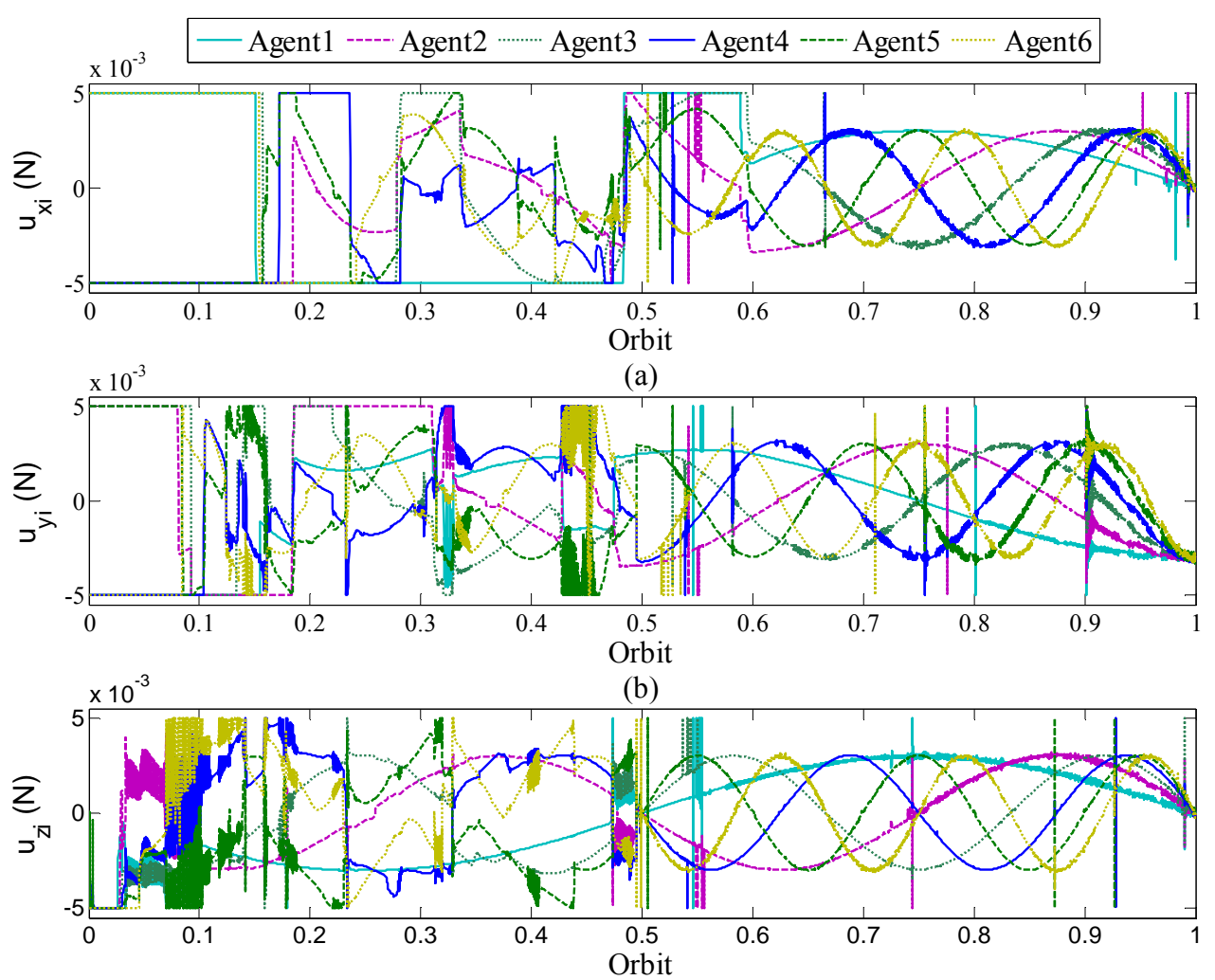

(c)

Figure 5.16: Control input (with $5 \mathrm{mN}$ thruster saturation)

Due to the consensus algorithm, the estimate error for each agent is affected by the other; Figure 5.17 is the best explanation. Before 0.2 of an orbit, agent 1 has a minimal estimate error, however, due to the directed communication link between agent 1 and agent 2, the estimate error in agent 2 has influenced agent 1 and agent 3. A similar situation applied to agent 4 and agent 5 . Agent 6 is furthest away from the sources of error, therefore, its estimate error remains lowest. The proposed control law is best fit for multi-agent decentralized systems. The estimated system constantly learns from the state of the plant, when tracking error in one agent propagates to another, the estimated system responds continuously. 


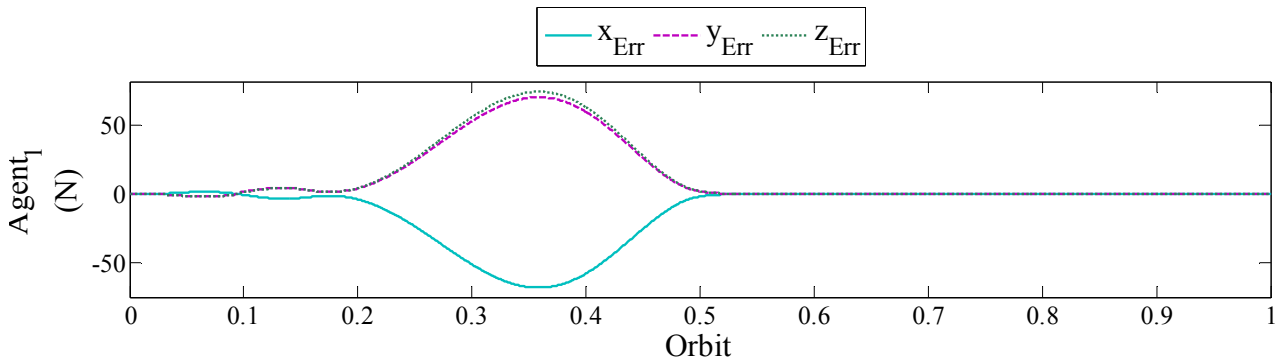

(a)

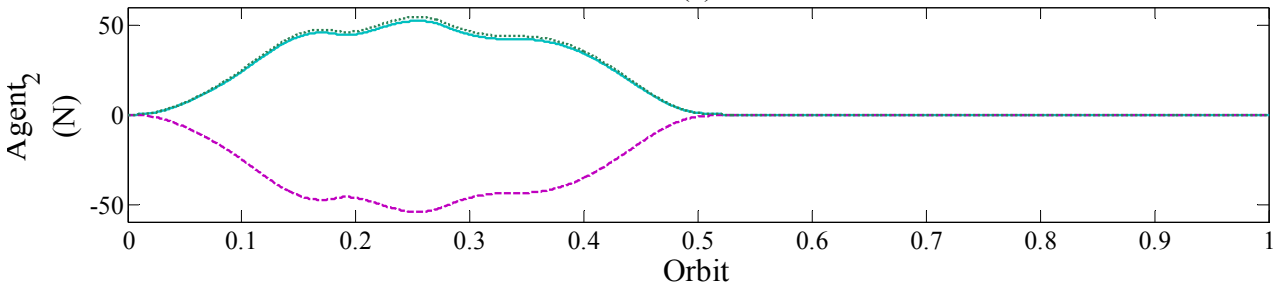

(b)

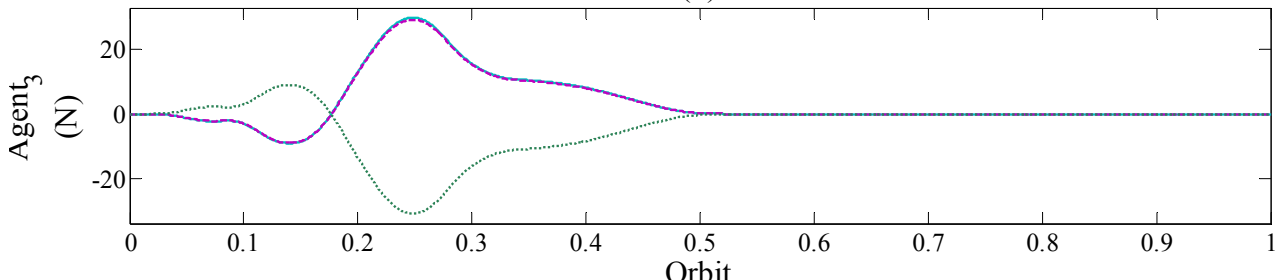

(c)

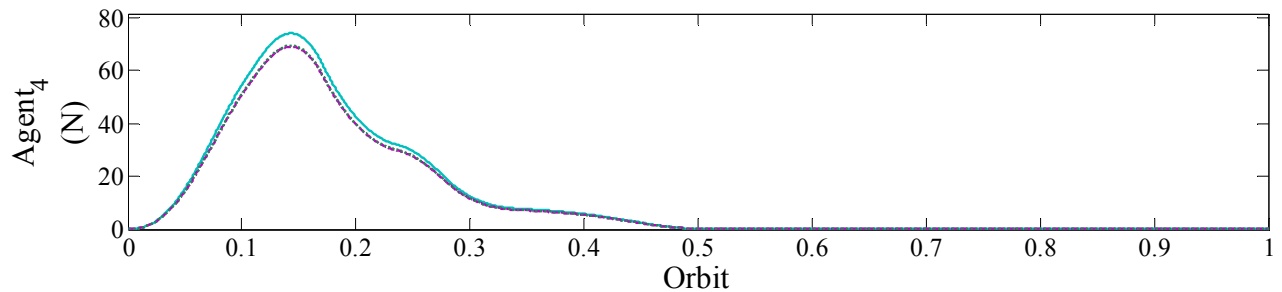

(d)

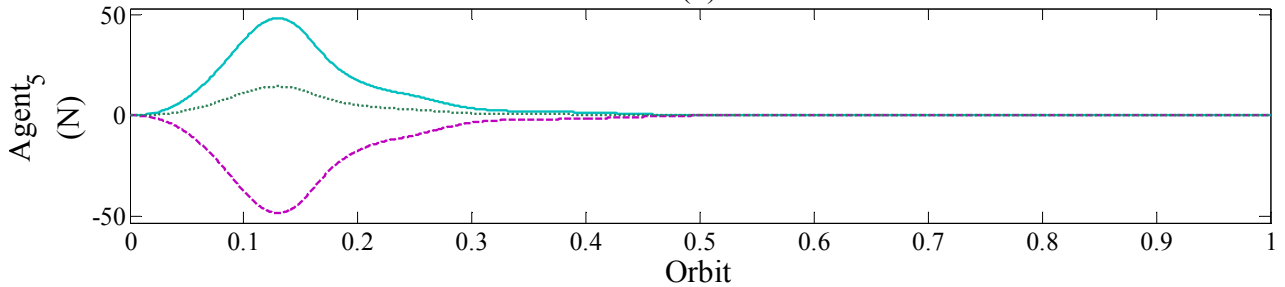

(e)

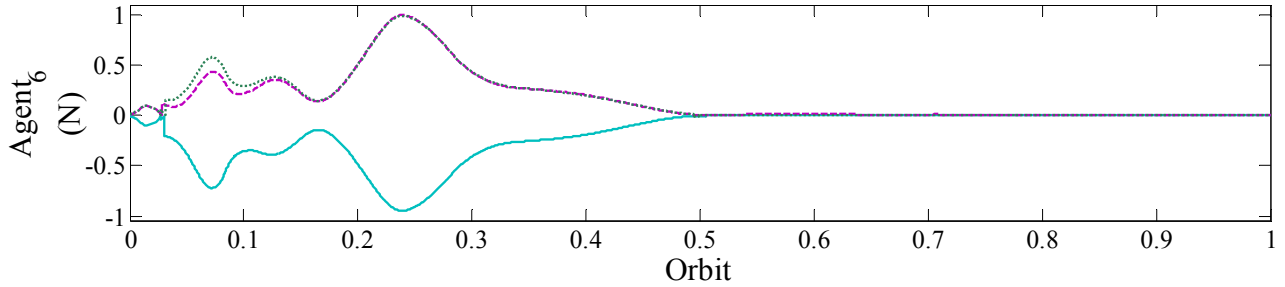

(f)

Figure 5.17: Estimated errors, large initial state error 


\subsubsection{Effect of Combining Sensor and Actuator Faults}

In Chapter 3 sensor faults are examined, in Chapter 4 actuator faults are investigated. In both chapters, the corresponding proposed control law is capable of encountering the faults from the system and fulfilled with the mission requirement. Except that the system did not converge within an orbit after the actuator faults were introduced into the system. Sensors faults and actuator faults have been examined individually for the adaptive robust control. Similar results have been obtained but better. The results shown below are of a much more challenging scenario, where both actuator and sensor faults are introduced at the same time. The system is able to be stabilized in a single orbit.

The actuator faults examined in this system are identical to the one examined in Chapter 4, the actuator forces for agents 1,5 , and 6 is set to be zero, agents 2 and 4 are set to be in reversed sign, and agent 3 has a constant $2 \mathrm{mN}$ thrust during the downtime. Alongside with the actuator faults are sensor faults. All 3 sensors on each agent outputs a reversed sign value. The duration of the downtime is 5 minutes, and it starts at 0.2184 of an orbit, and ceases at 0.3822 of an orbit.
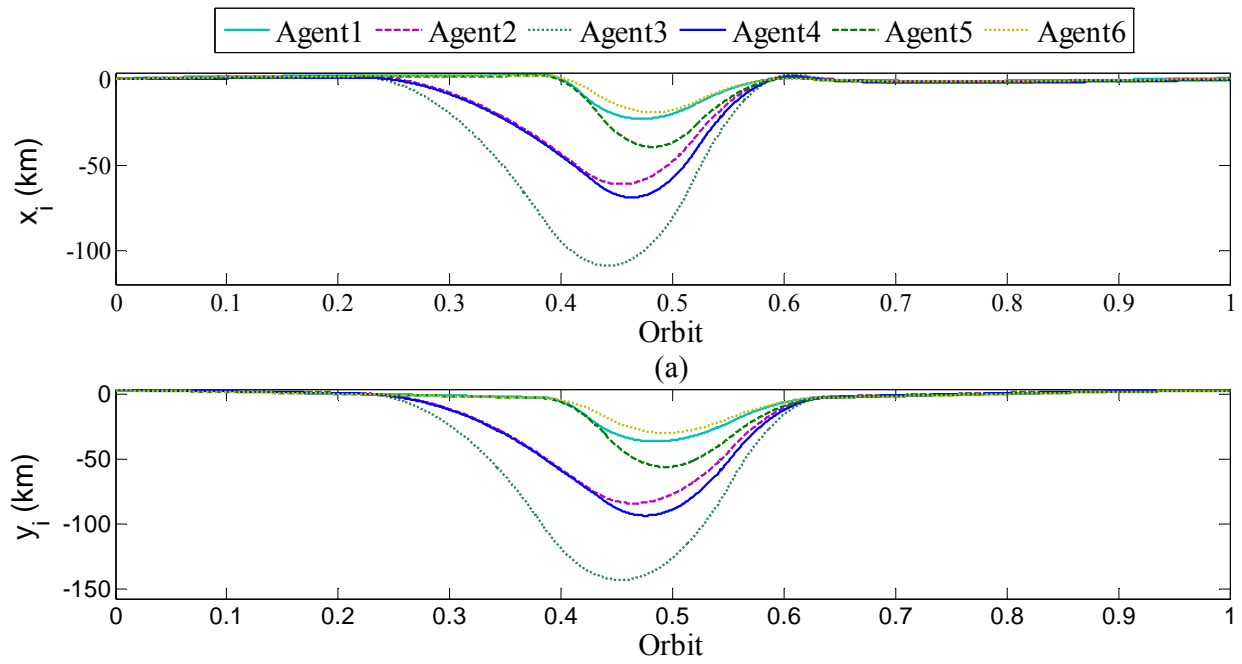

(b)

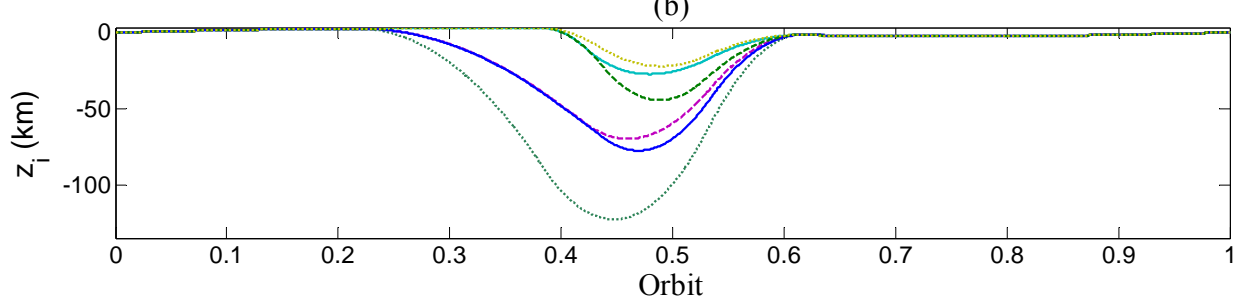

(c)

Figure 5.18: State of all agents, multiple faults (without disturbance) 
As learnt from Chapter 4, actuator faults lead to more system state error than sensor faults. Since the actuator forces are set to zero for agents 1, 5, and 6, therefore, it is reflected in Figure 5.18 that those three lines in all three directions are much less.
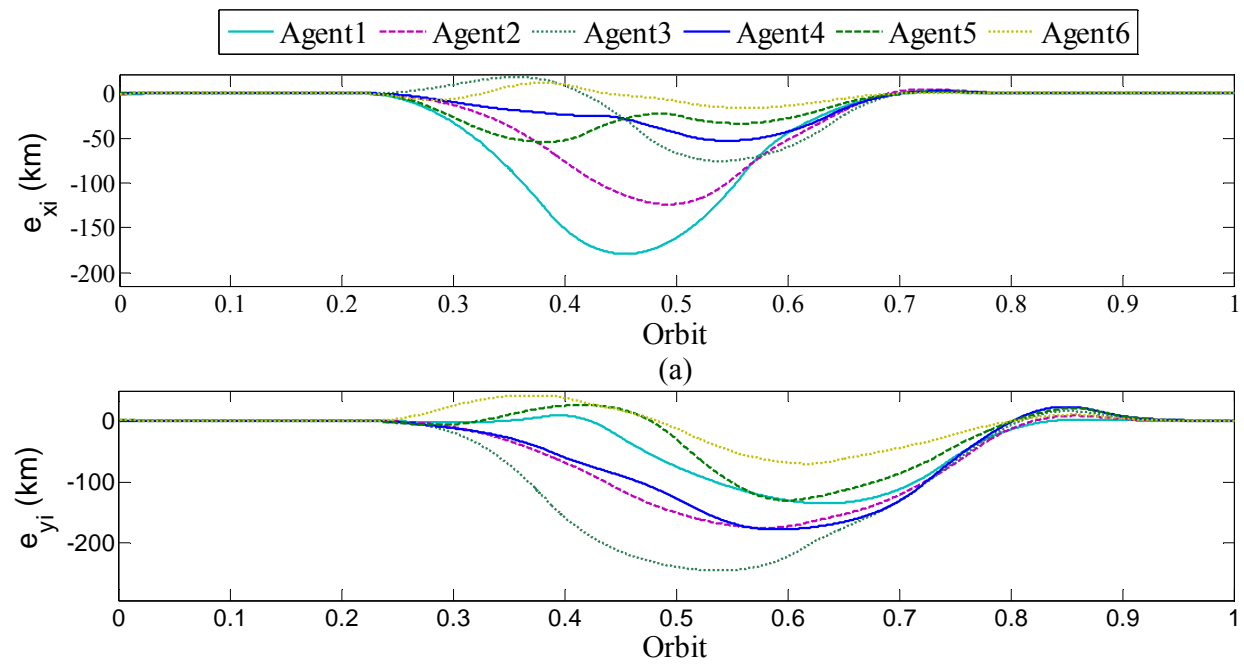

(b)

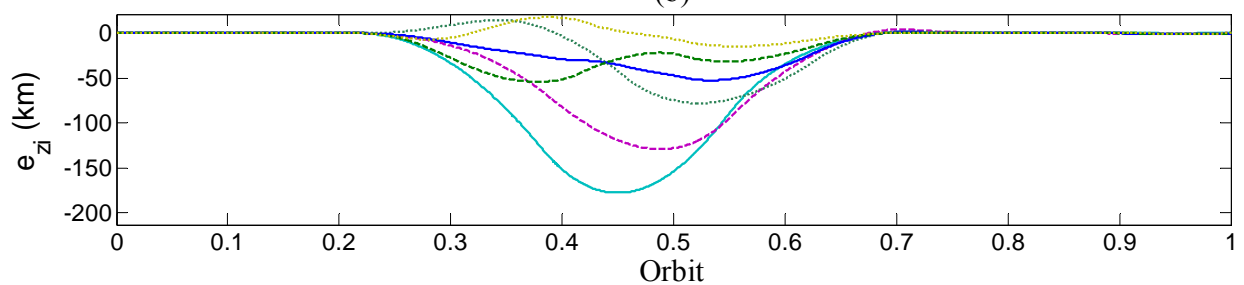

(c)

Figure 5.19: Tracking errors, multiple faults (with disturbance)

The tracking error shown in Figure 5.19 is the result where $3 \mathrm{mN}$ disturbance is applied when actuator and sensors faults are presented simultaneously. This result was not achievable for both the SMC and the SMC robust control.

The formation was affected greatly when disturbance was applied to the system, as depicted in Figure 5.20. However, for both scenarios, the formation is developed along with the convergence of the tracking error. The maximum allowable thrust is $5 \mathrm{mN}$ per agent, since there are 6 agents in the system, the maximum value for OCEM is $0.03 \mathrm{~N}$. In Figure 5.21, for both scenarios, the maximum OCEM is no more than $0.02 \mathrm{~N}$, therefore, not all agents have reached actuator saturation points even when multiple faults were applied. 


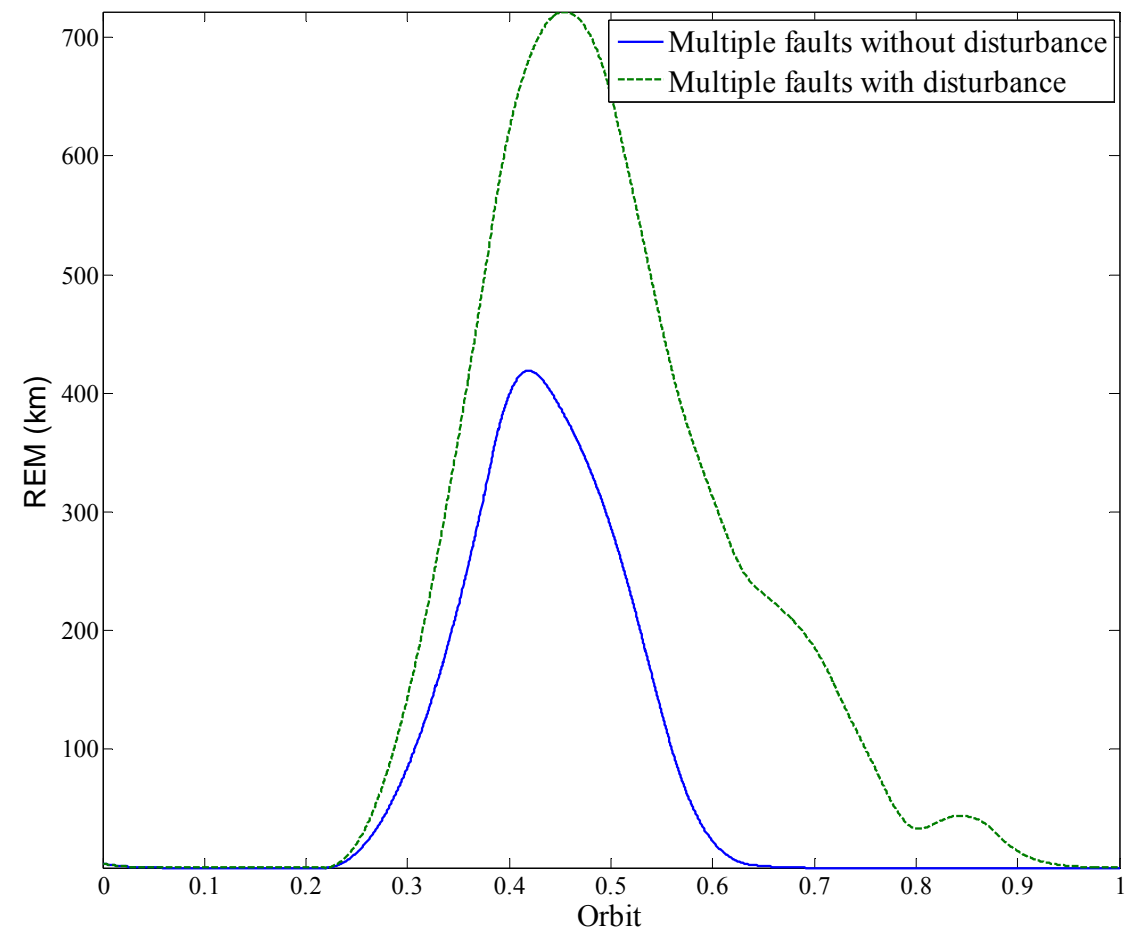

Figure 5.20: REM, multiple faults

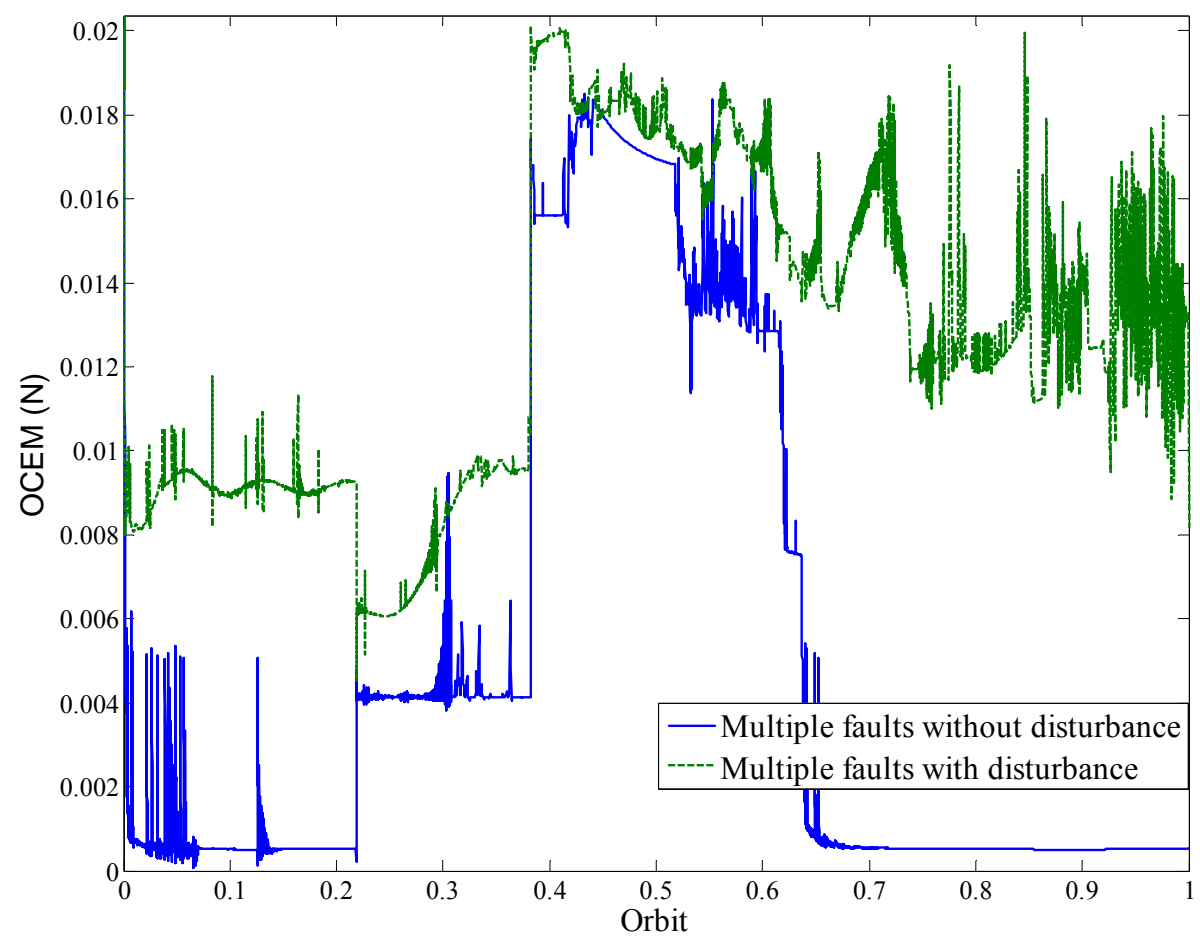

Figure 5.21: OCEM, multiple faults 

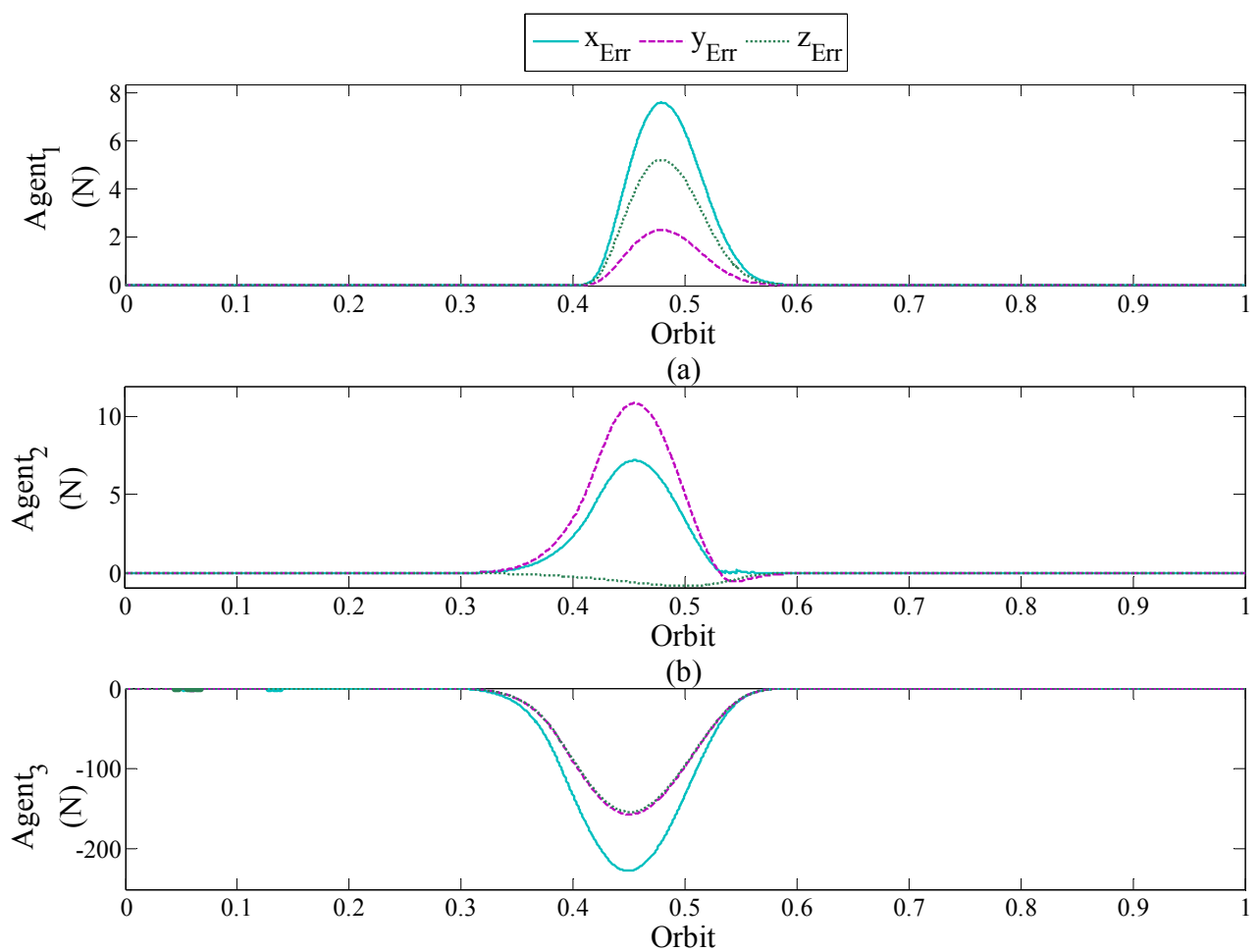

(c)

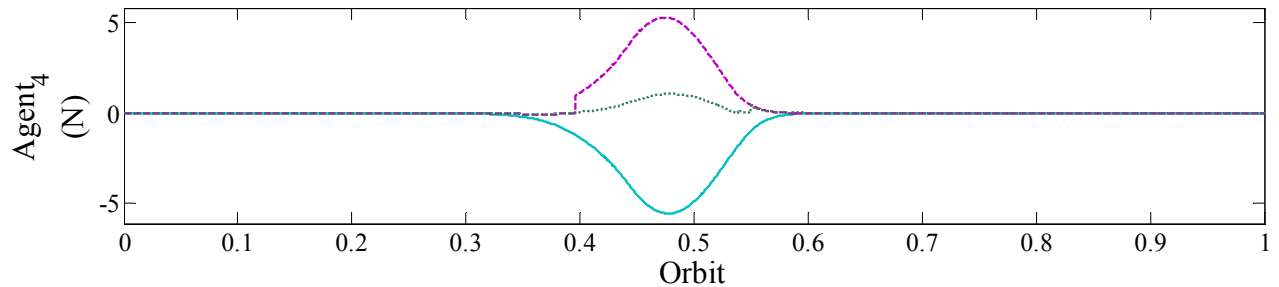

(d)

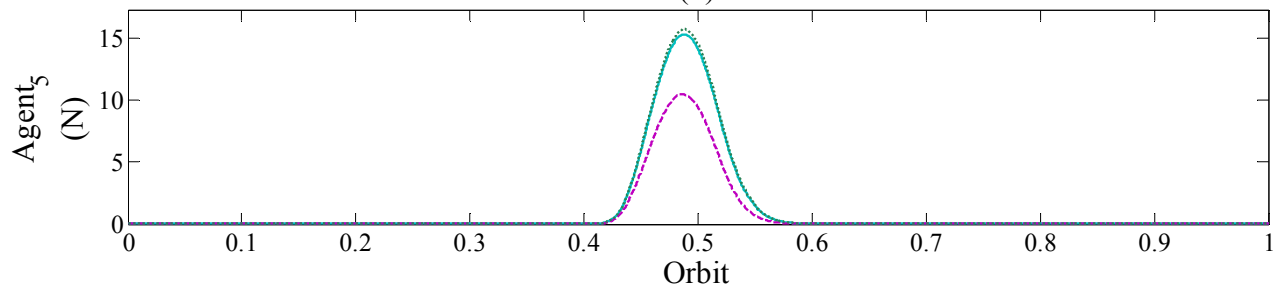

(e)

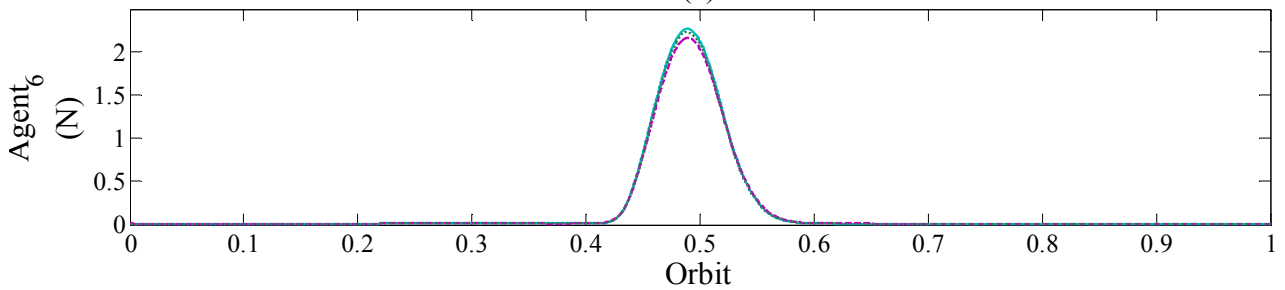

(f)

Figure 5.22: Estimated errors, multiple faults (without disturbance) 
The estimate errors vary depending on the location of the agent and it is also influenced by the state error. The state error is much larger with disturbances when multiple faults are applied, hence the estimated error will also be larger than the one without disturbance.

The adaptive robust control law can handle both actuator and sensor faults at the same time. Moreover, with the presence of the disturbance that is equivalent to $60 \%$ of the maximum allowable thrust, the state error converged in one orbit. This control law is much effective than the SMC robust control developed in Chapter 4.

\subsubsection{Effect of Spacecraft Mass Variations}

Continuing with the discussion from Chapter 3.2.5 and 4.2.5, at similar phenomenon is examined again. In space missions, the mass of the spacecraft is a very important parameter. The mass of the spacecraft changes from time to time in the design process. Moreover, the mass of the spacecraft decreases during the mission when propellant were used. Therefore, a good control law is to work independently with the mass of the spacecraft. Adaptive control allows the control law to be able to control with unknown parameters without adjusting any control gains. Previously, a 2, 5, and $10 \mathrm{~kg}$ spacecraft mass were examined in Chapter 3; the results for the $10 \mathrm{~kg}$ were not stable. A 2 and $10 \mathrm{~kg}$ spacecraft were examined in Chapter 4, it shows that with robust control, the $10 \mathrm{~kg}$ mass is controllable. Then, a scenario is examined where the mass of the spacecraft varies during the mission. The mass of the spacecraft reduces from $10 \mathrm{~kg}$ to $1 \mathrm{~kg}$ in half of a reference orbit. The mass of the spacecraft decreases in a constant speed within the specific time. It is a good model simulating that the propellant is burned from the beginning of the mission and is done within half of the reference orbit. $10 \mathrm{~kg}$ is a satisfactory mass when designing a control law for nano scale spacecraft, and in Chapter 4 the robust control has more than enough power to control this situation. However, with the new control law, mass will not be a critical parameter.

The estimated value for the CNN system does not match the previous scenario, the estimate error is shown in Figure 5.23. However, error in estimation does not mean the system is not controlled. The control system responds to the estimated value and provide sufficient control effort to bring all agents to their desired trajectory. 


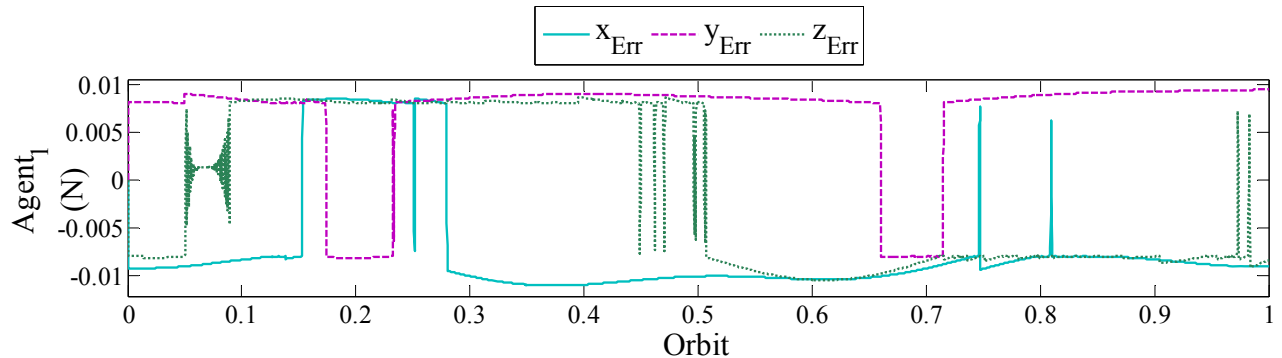

(a)

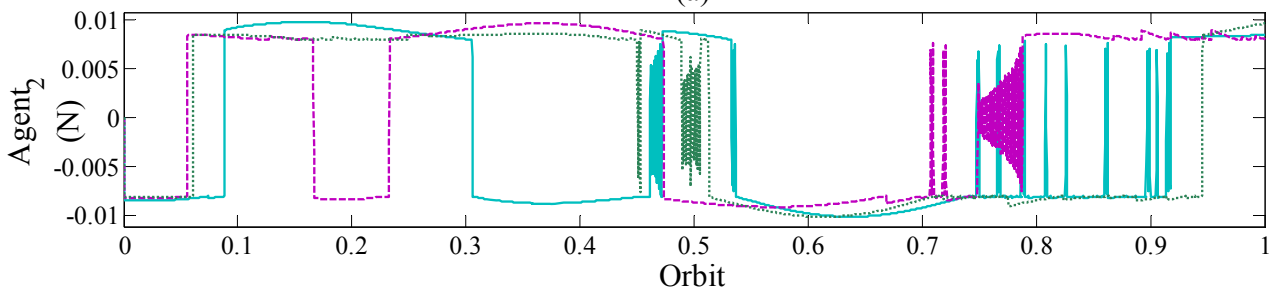

(b)

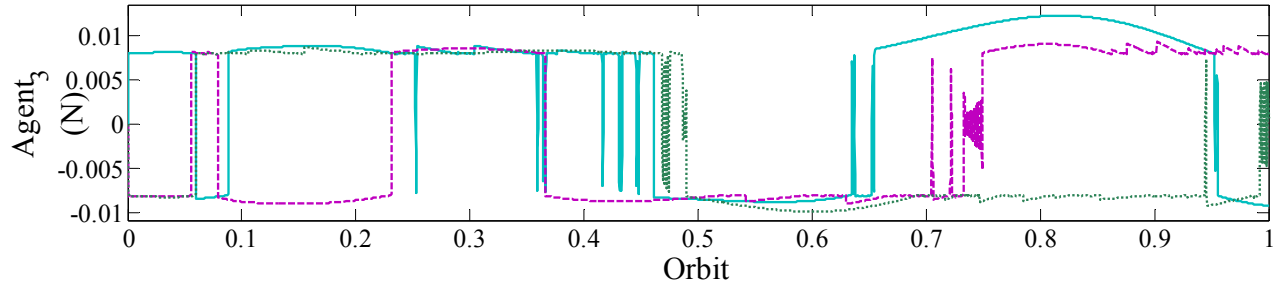

(c)

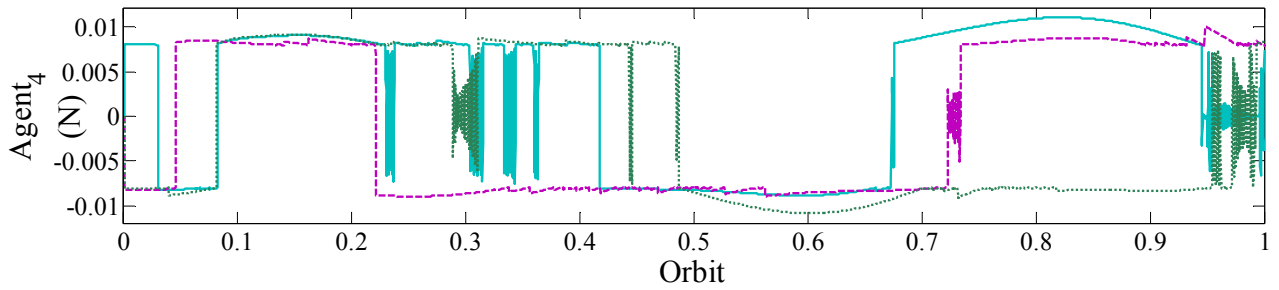

(d)

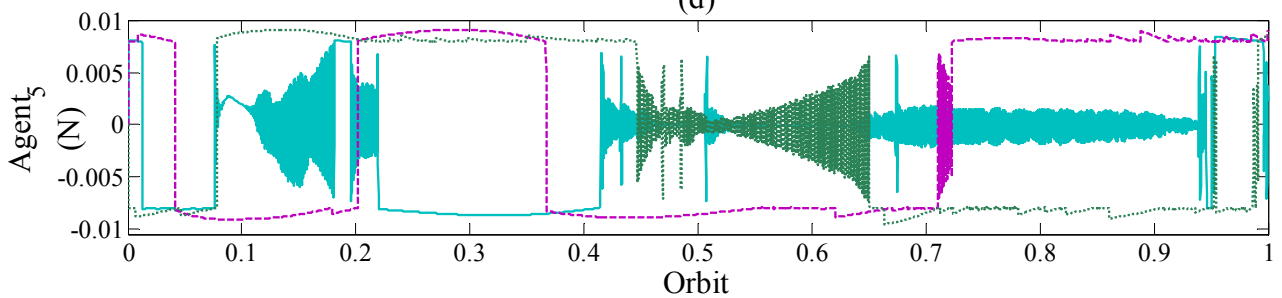

(e)

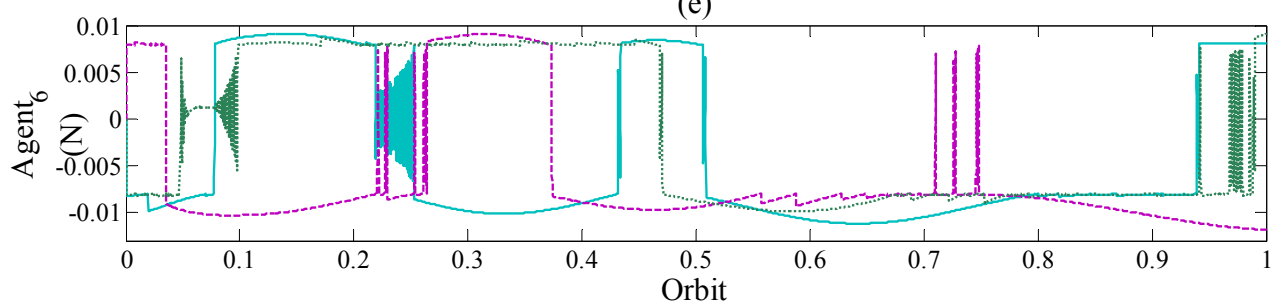

(f)

Figure 5.23: Estimated errors, spacecraft mass $=20 \mathrm{Kg}$ 
With $5 \mathrm{mN}$ of maximum actuator force available, the robust SMC maintains a steady state error that cannot be eliminated in both AEM and REM, see Figure 5.24 and Figure 5.25. In addition, the mass of the spacecraft in the simulation using the CNN adaptive control law is double that of the mass of the other.

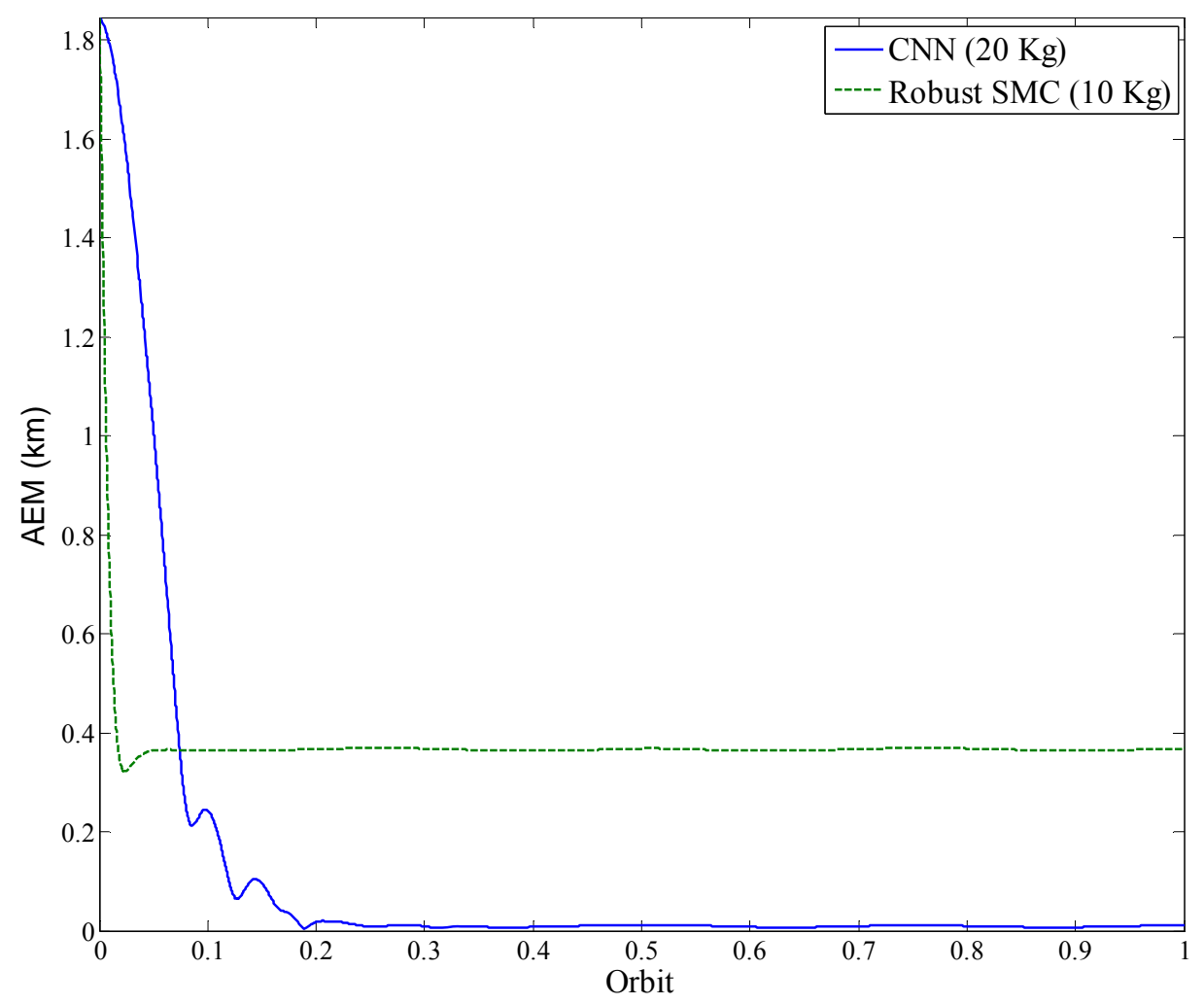

Figure 5.24: AEM, CNN vs robust SMC

A $50 \mathrm{~kg}$ scenario is examined, similar AEM and REM results are obtained. However, longer settling time is needed due to the limited actuator force. The learning process in the estimated system has reached its highest state similar to Figure 5.23. More control effort is needed to compensate for the extra mass from the system but it is controlled, see Figure 5.26. The multiagent system is able to reach tracking consensus after 0.77 of an orbit. 


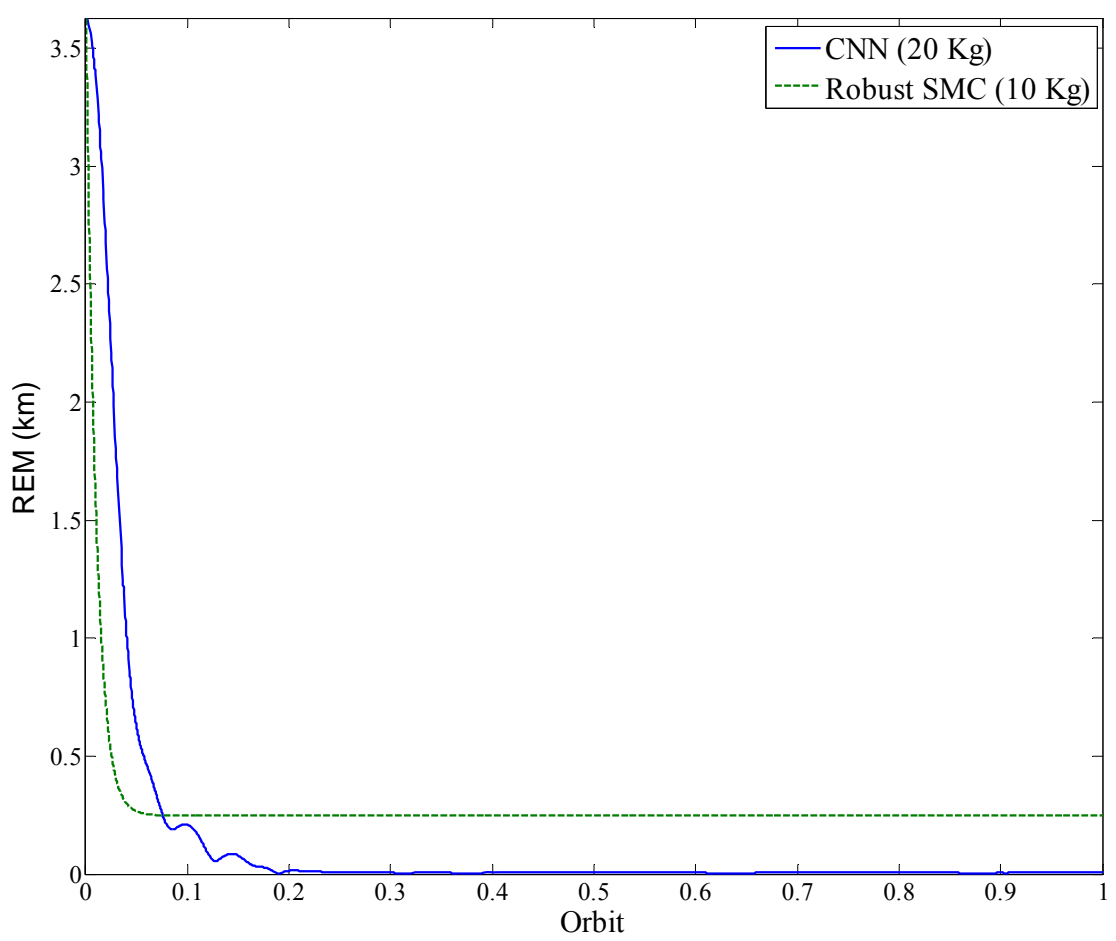

Figure 5.25: REM, CNN vs robust SMC

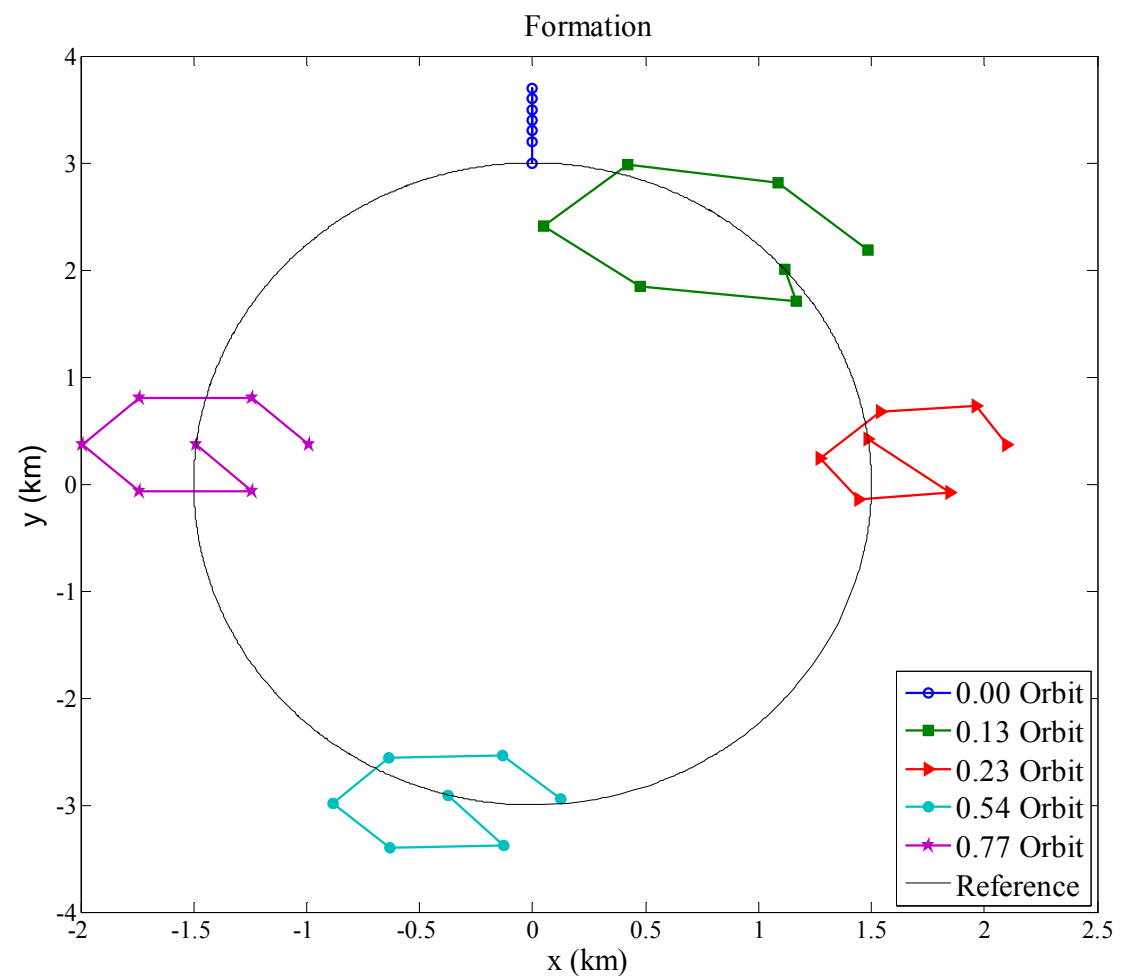

Figure 5.26: System state snap shot at different time, spacecraft mass $=50 \mathrm{~kg}$ 


\subsubsection{Effect of Formation Reconfiguration}

Orbital reconfiguration is a very practical research topic but yet much less effort has been put toward this. With the advancements in adaptive control, it is more feasible to perform this reconfiguration during a mission. Adaptive control allows the control system to respond to the dynamic change without manual adjustment in control parameters. If modelled correctly, the control system should be able to adapt to the changes and approach to the new state smoothly. The key to this change is that the new reference trajectory has to work within the maximum thrust.

When an orbit period is reduced, the required system control input increases, see Figure 5.27. The period of the reference trajectory is halved after the first orbit, and the required input force is more than double.
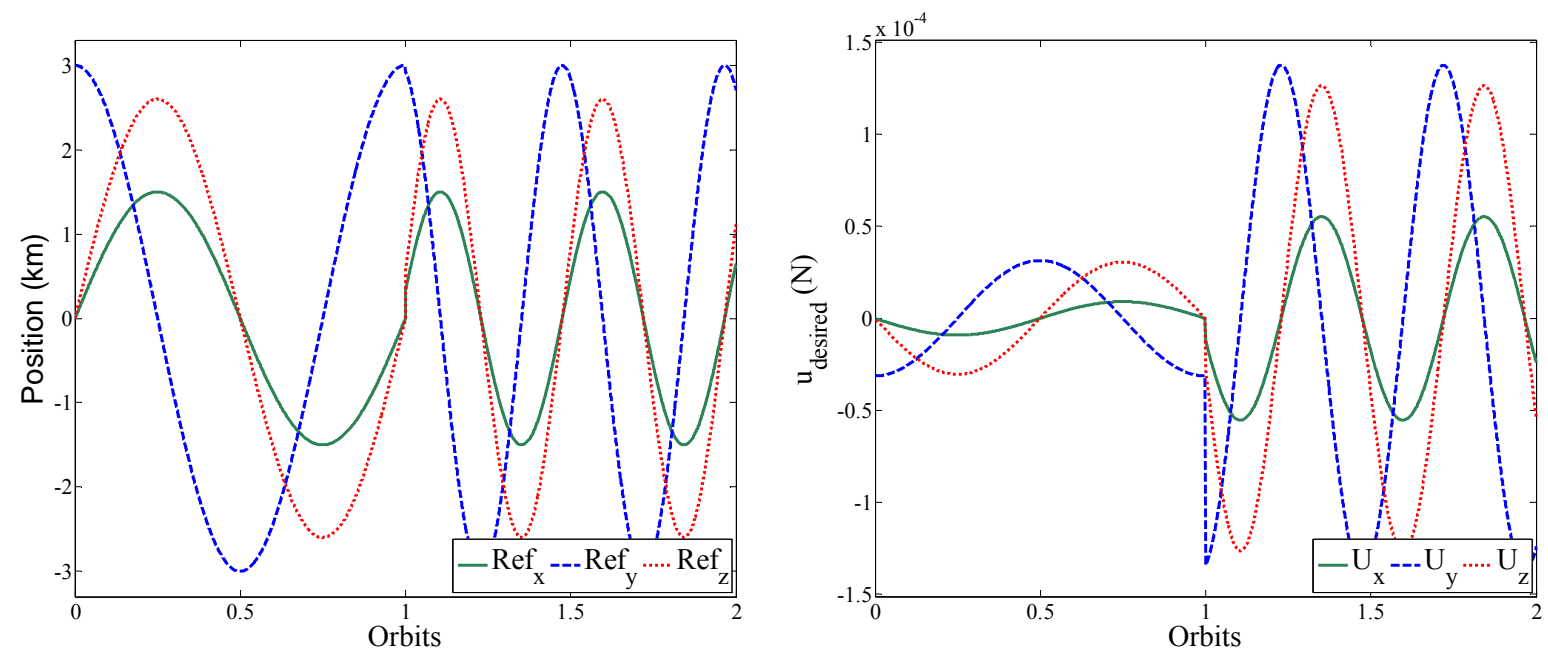

Figure 5.27 Reference trajectory and minimum control input required

With the proposed CNN adaptive robust control law, the system responds smoothly to the change and it is able to reduce the state error is a short period of time, Figure 5.28. Furthermore, the relative error between each agents is converged. The system control input saturated due to the sudden change in orbital speed, however, it was brought back to normal in less than 0.05 of an orbit after the reconfiguration. The error in estimation is very minimum, and the estimated system quickly adapts to its change. 


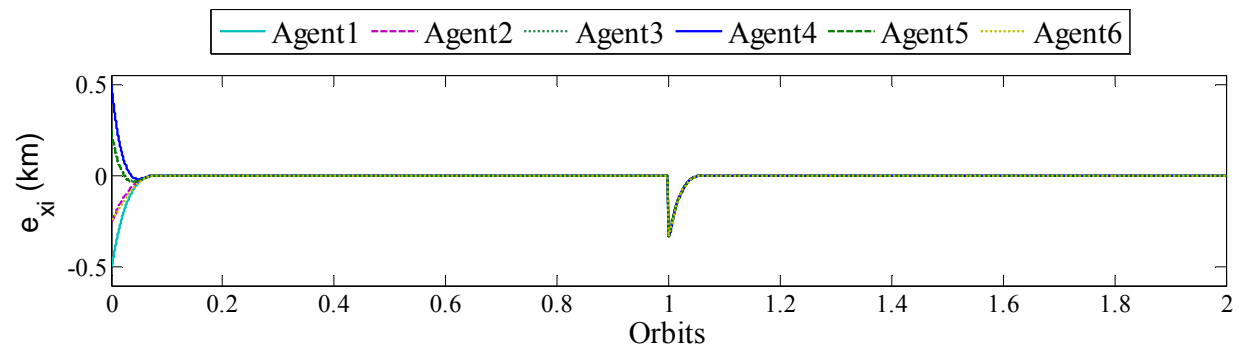

(a)

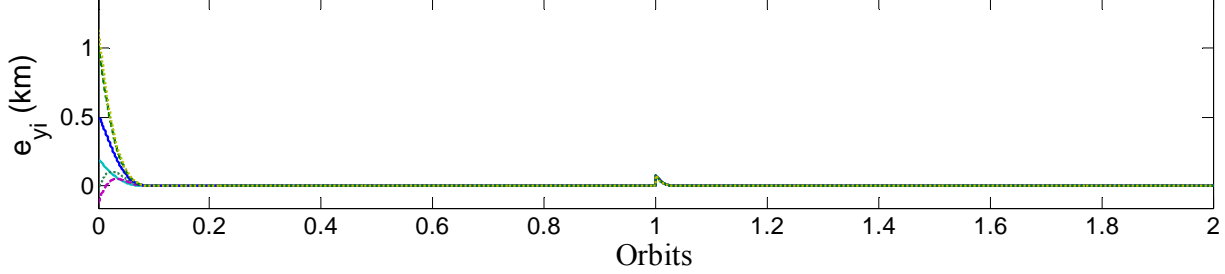

(b)

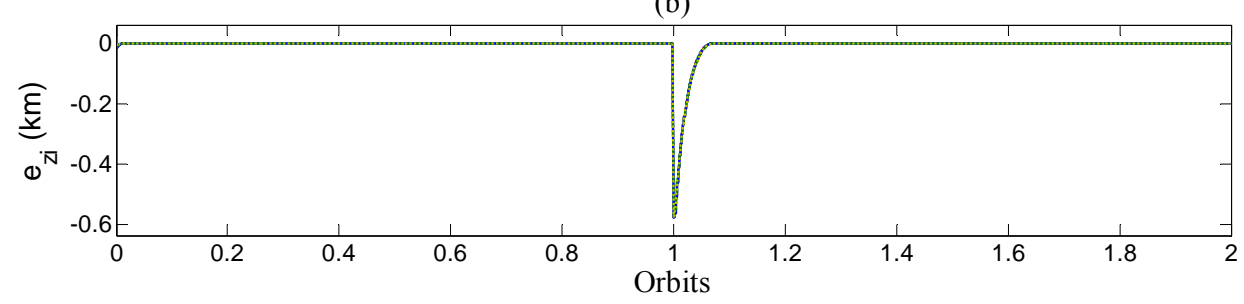

(c)

Figure 5.28: Tracking errors, halved orbit period

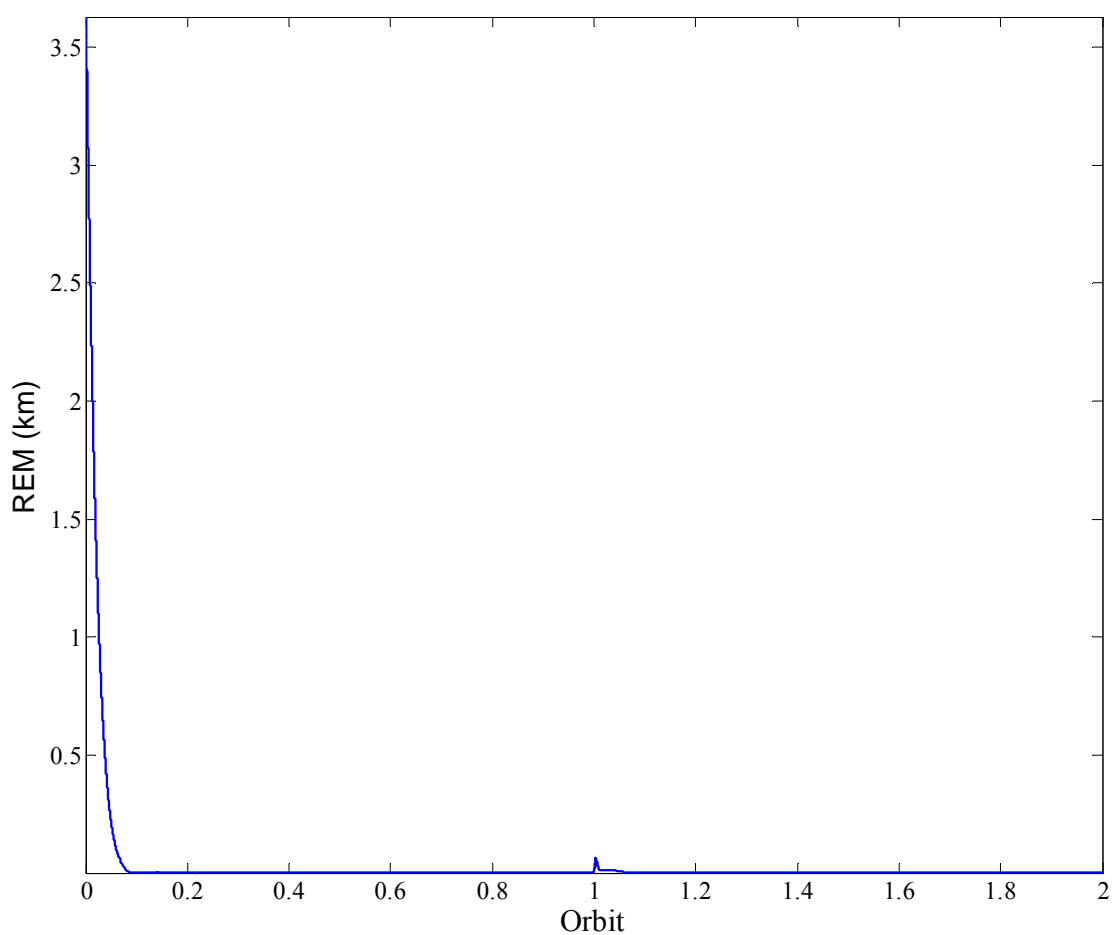

Figure 5.29: REM, halved orbit period 
The next configuration in line is changing both the orbital period and the shape of the orbit. This time the orbital period is still to be halved, and the reference trajectory changes from elliptical to a circular trajectory while keeping the radius in the $\mathrm{x}$-direction the same, see Figure 5.30 and Figure 5.31.
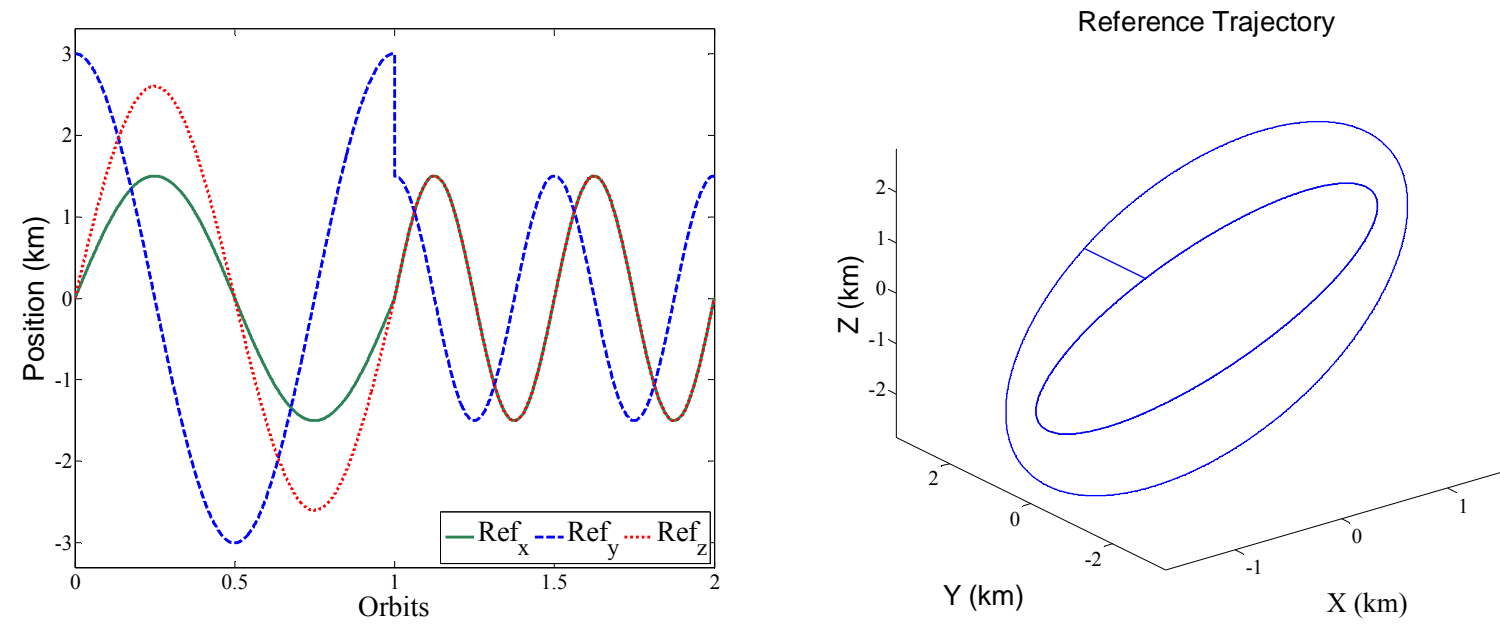

Figure 5.30 Reference trajectory, elliptical to circular orbit

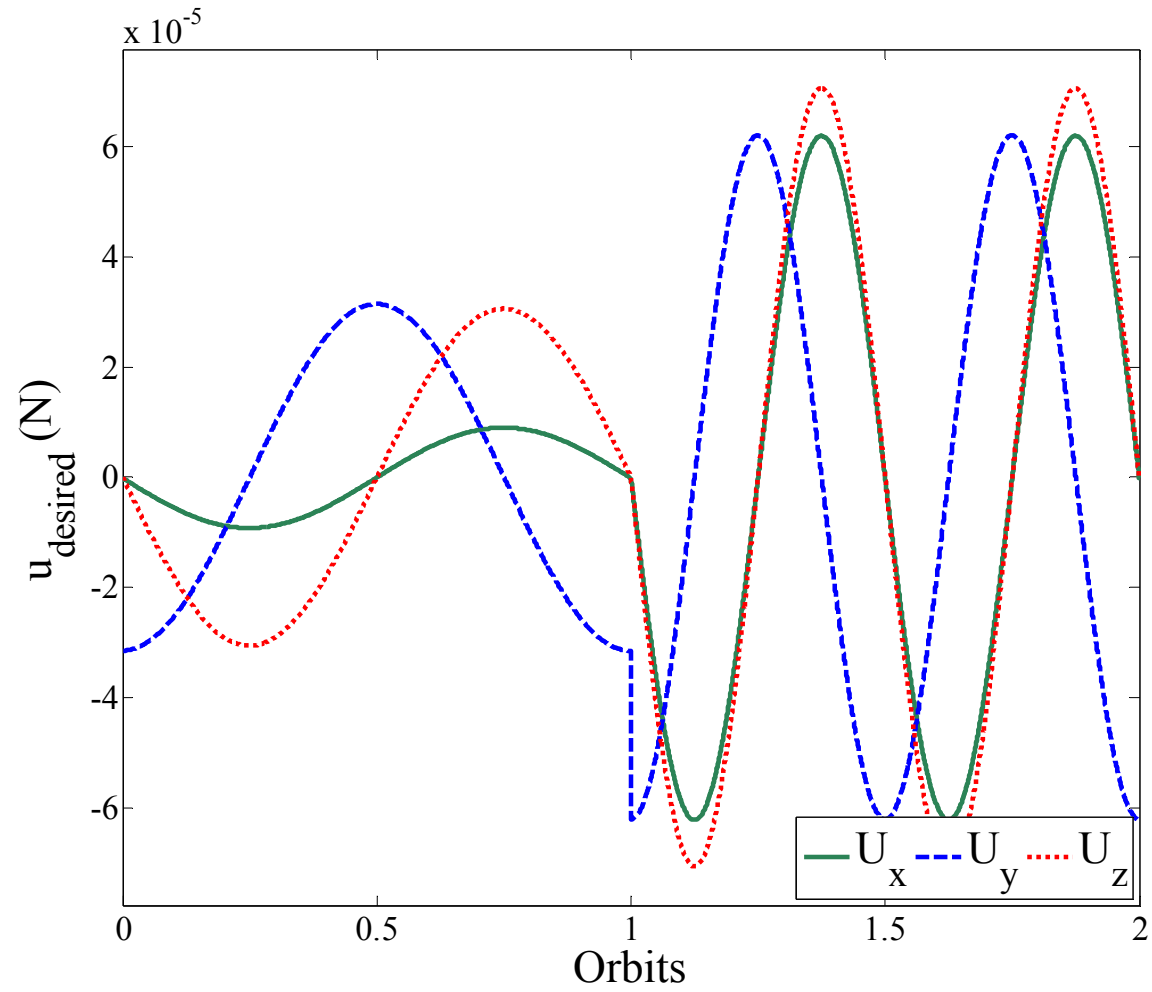

Figure 5.31 Minimum control input required, elliptical to circular orbit 


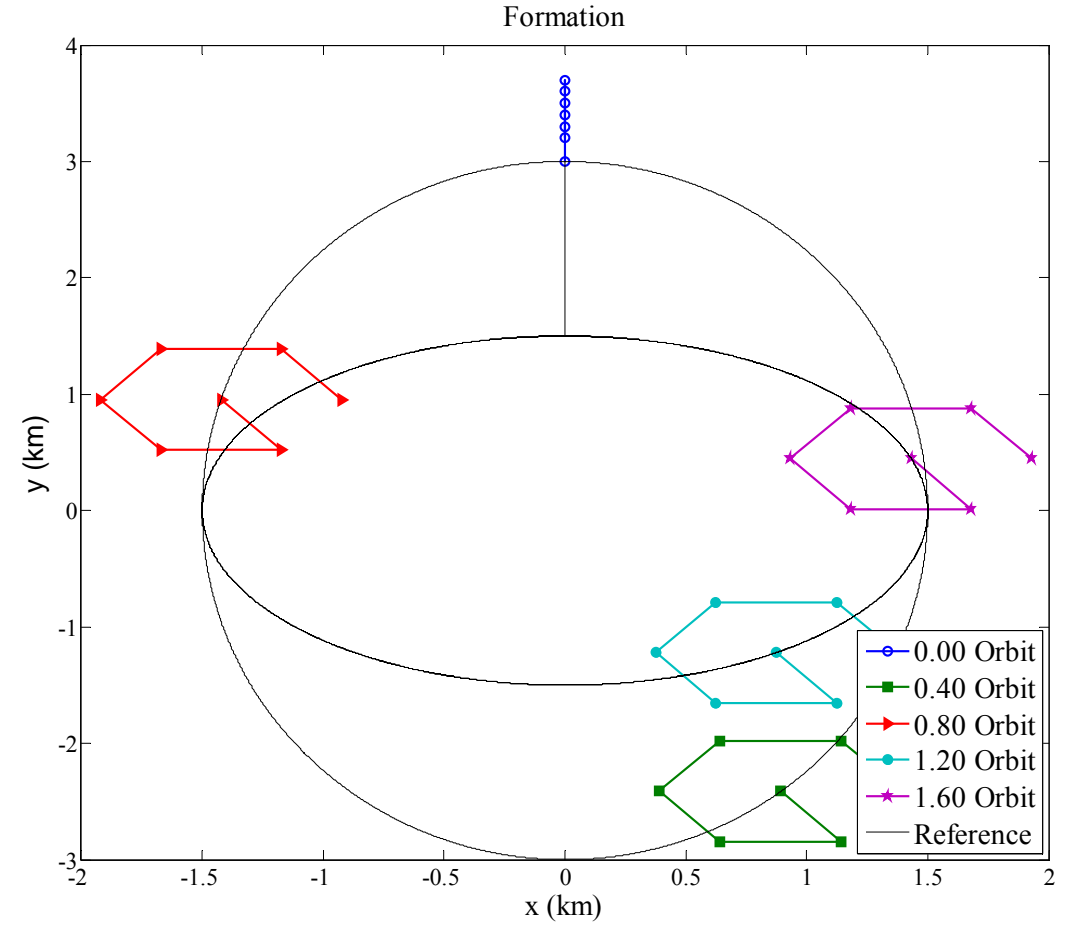

Figure 5.32: System state snap shot at different time, elliptical to circular orbit

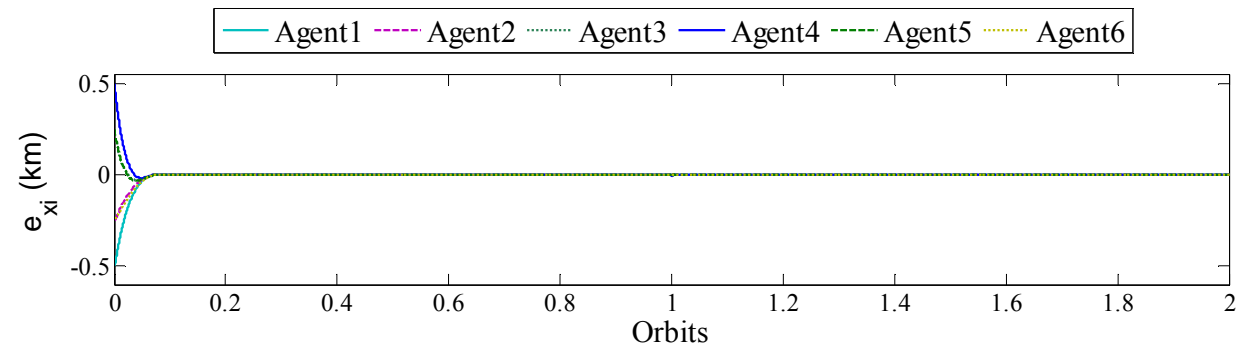

(a)
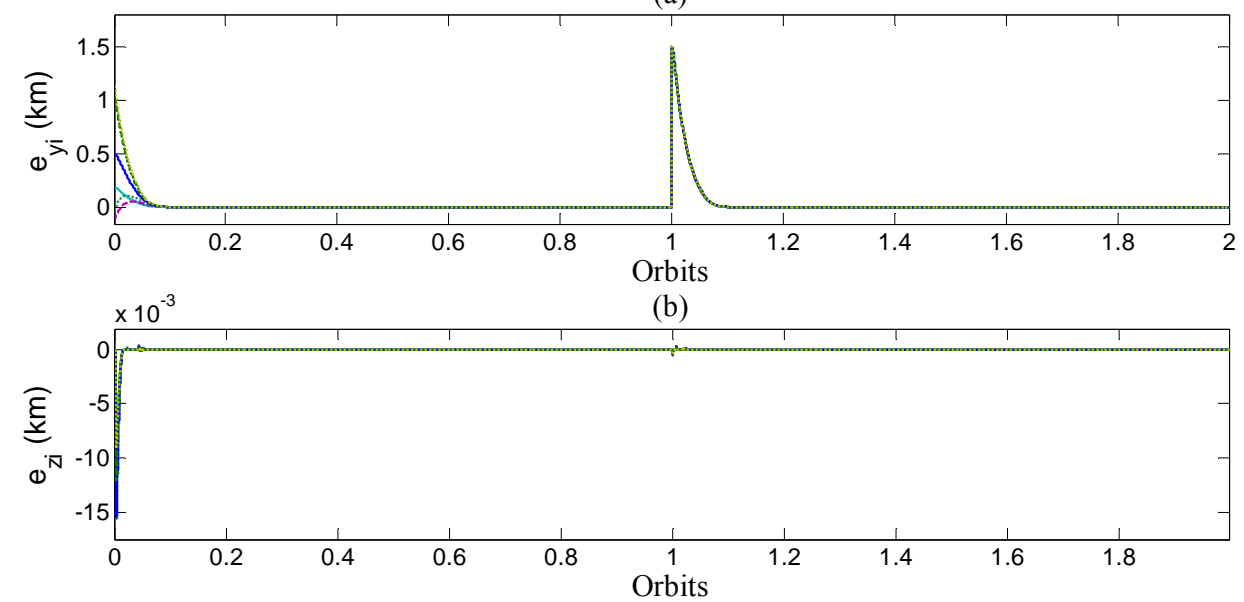

(c)

Figure 5.33: Tracking errors, elliptical to circular orbit 
The system responds very similar to the previous scenario. The REM has a similar profile as before, the formation of the agents moves toward the new trajectory together, Figure 5.32. However, the state error is slightly higher due to the greater change in reference path, Figure 5.33. The control required, Figure 5.34, for this change is very similar to the previous scenario.
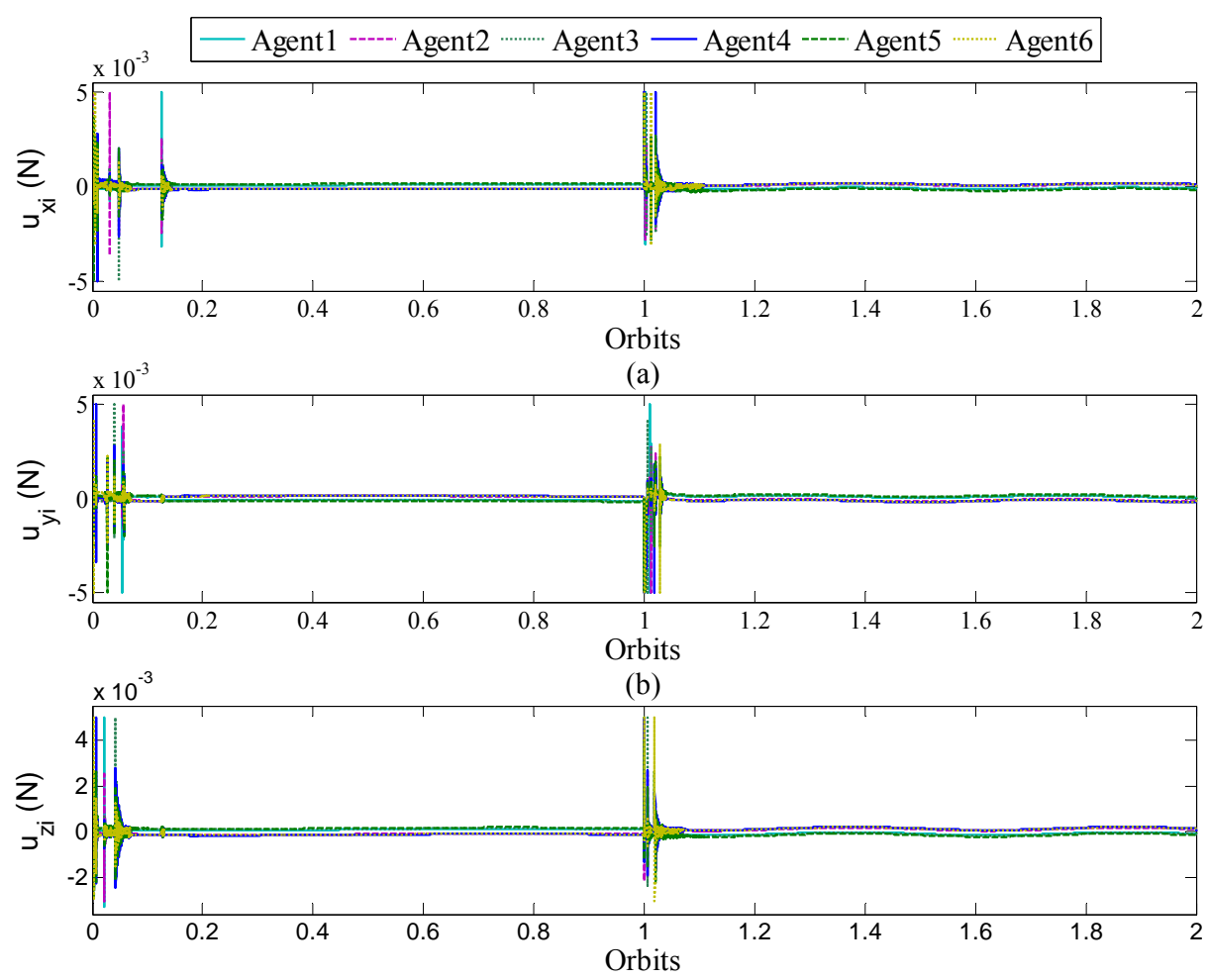

(c)

Figure 5.34: Control input, elliptical to circular orbit

The following results comprised all 3 possible orbital reconfigurations. The mission first starts with the old elliptical orbit, then the period is halved while changing to a circular orbit. Lastly, the formation is changed from a planar regular hexagon to a three-dimensional pyramid as described in Chapter 5.2.1. In Figure 5.35, at 1.60 of an orbit is where the pyramid formation is in a planar view. The system state graph, Figure 5.36, clearly shows that the shape and the period of the orbit have changed after the first orbit. At 1.5 of an orbit, it is where the formation reforms. Agent 1 to 4 share the same $\mathrm{x}$ value; agent 2,3, agent 1, 4 and agent 5, 6 has the same y value; similar trend occurred in the z-direction. The system control input are less than $5 \mathrm{mN}$, and the state error for each agent converged quickly to all changes. 


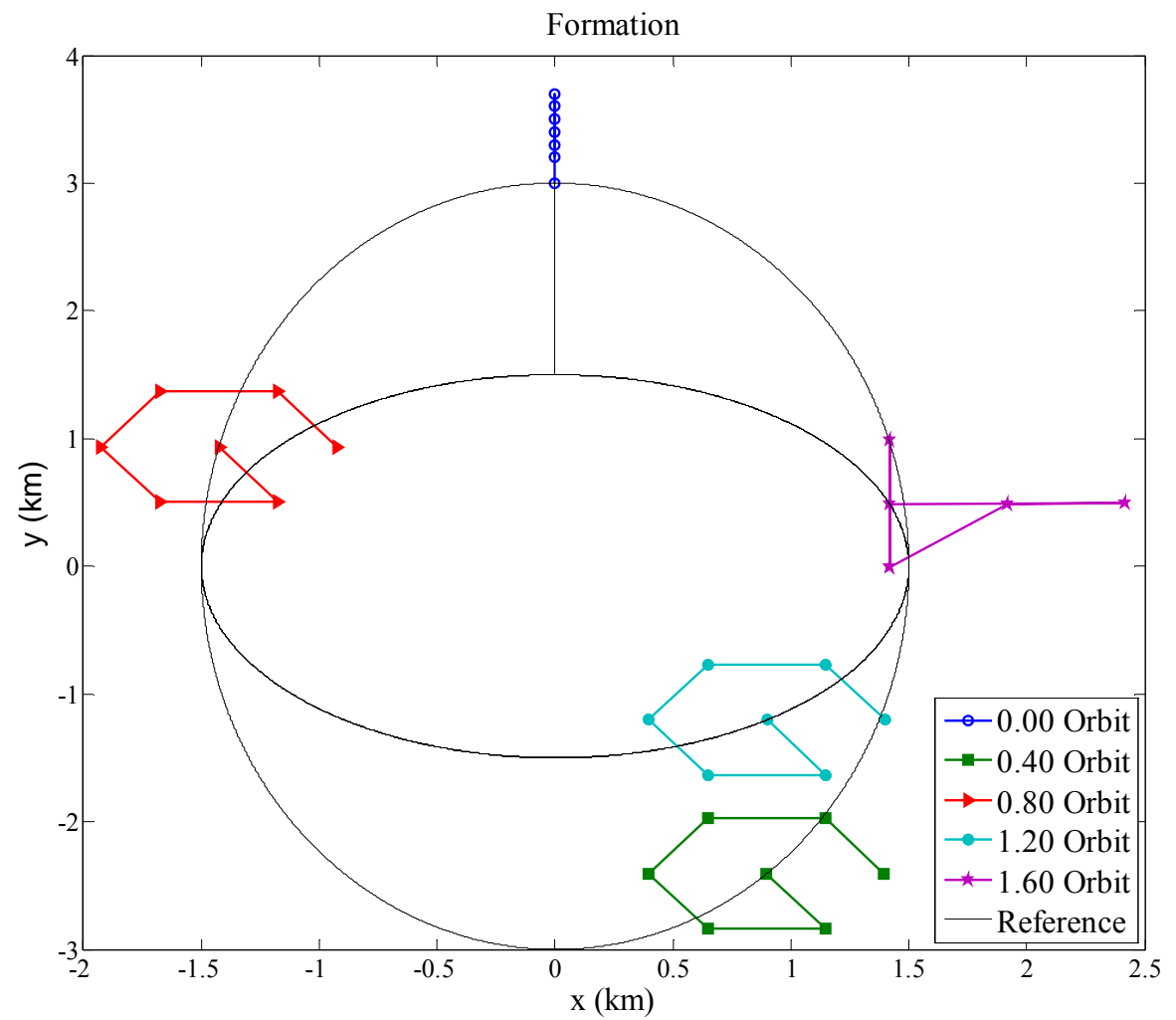

Figure 5.35: System state snap shot at different time, formation reconfiguration
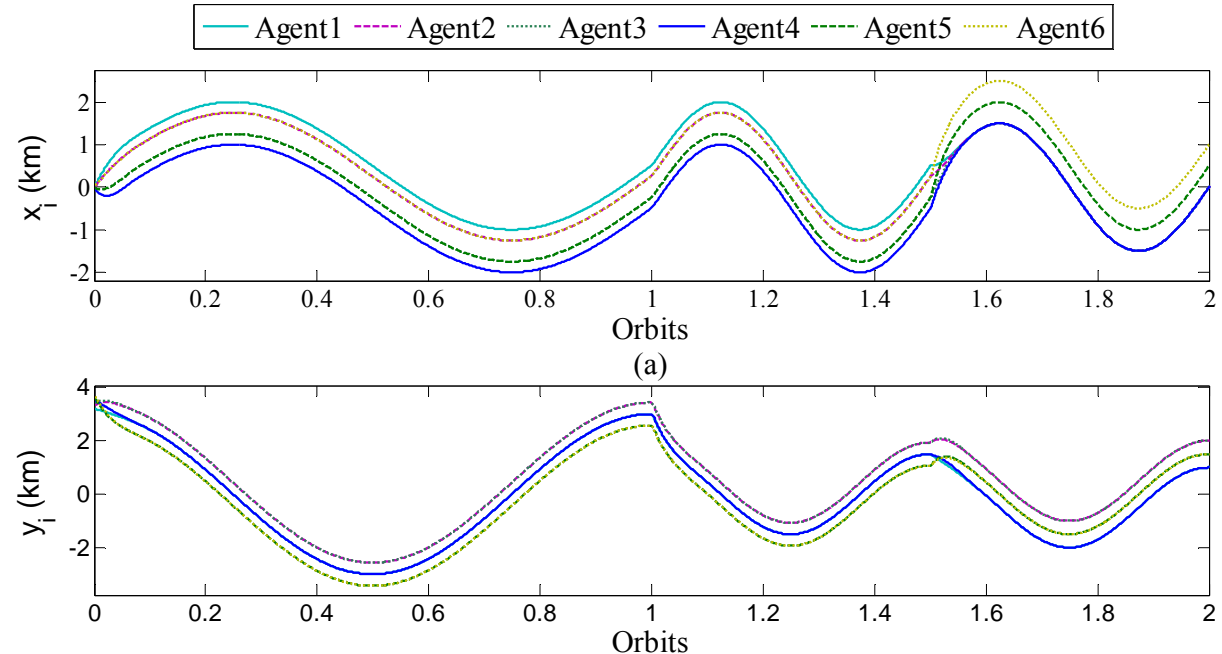

(b)

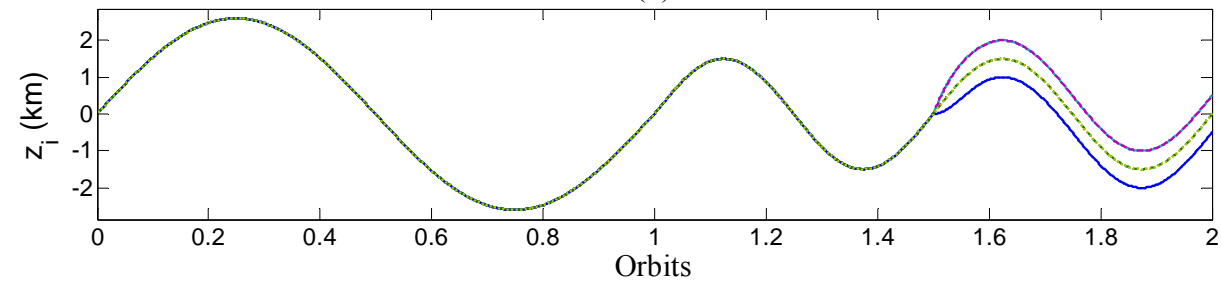

(c)

Figure 5.36: State of all agents, formation reconfiguration 

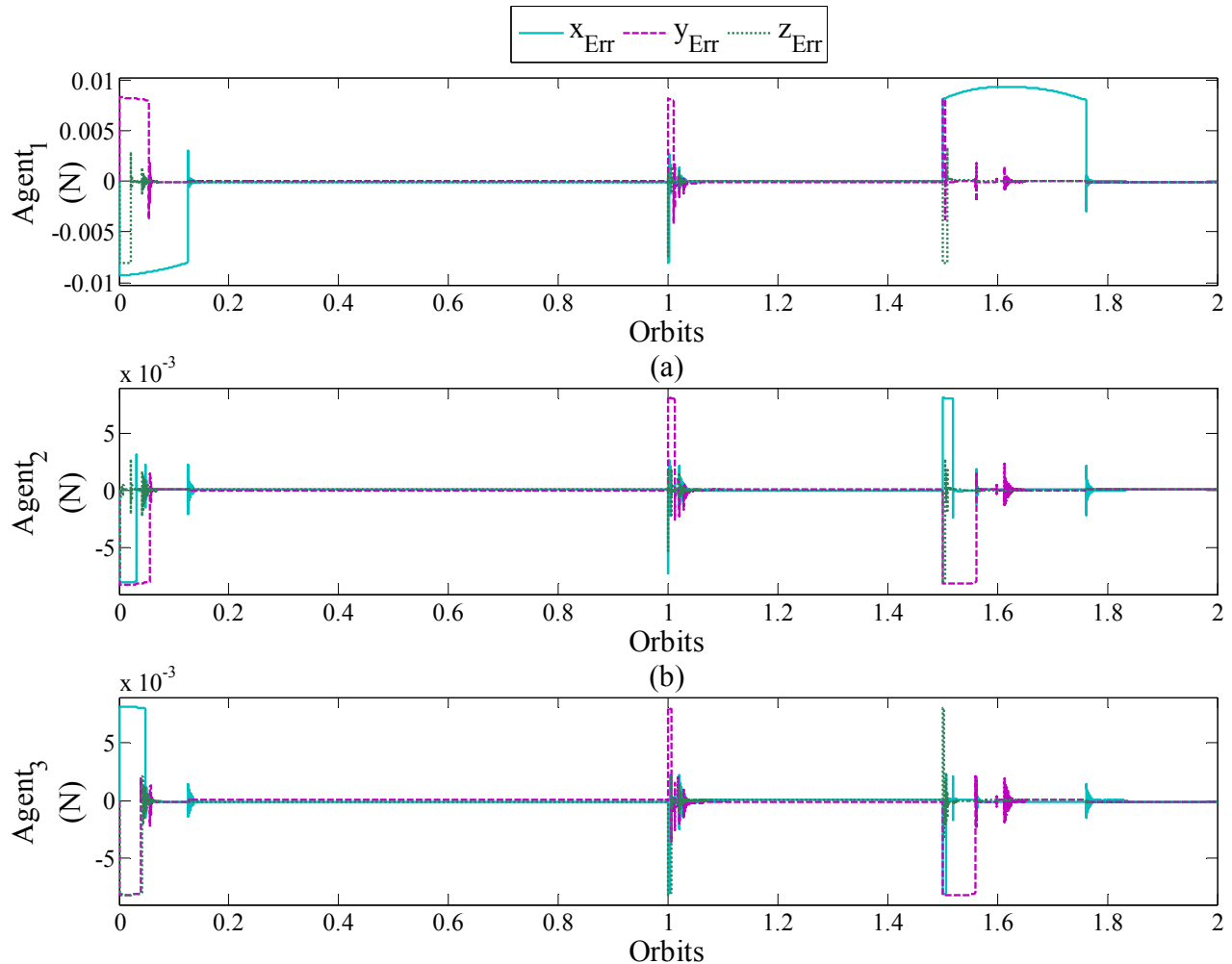

(c)
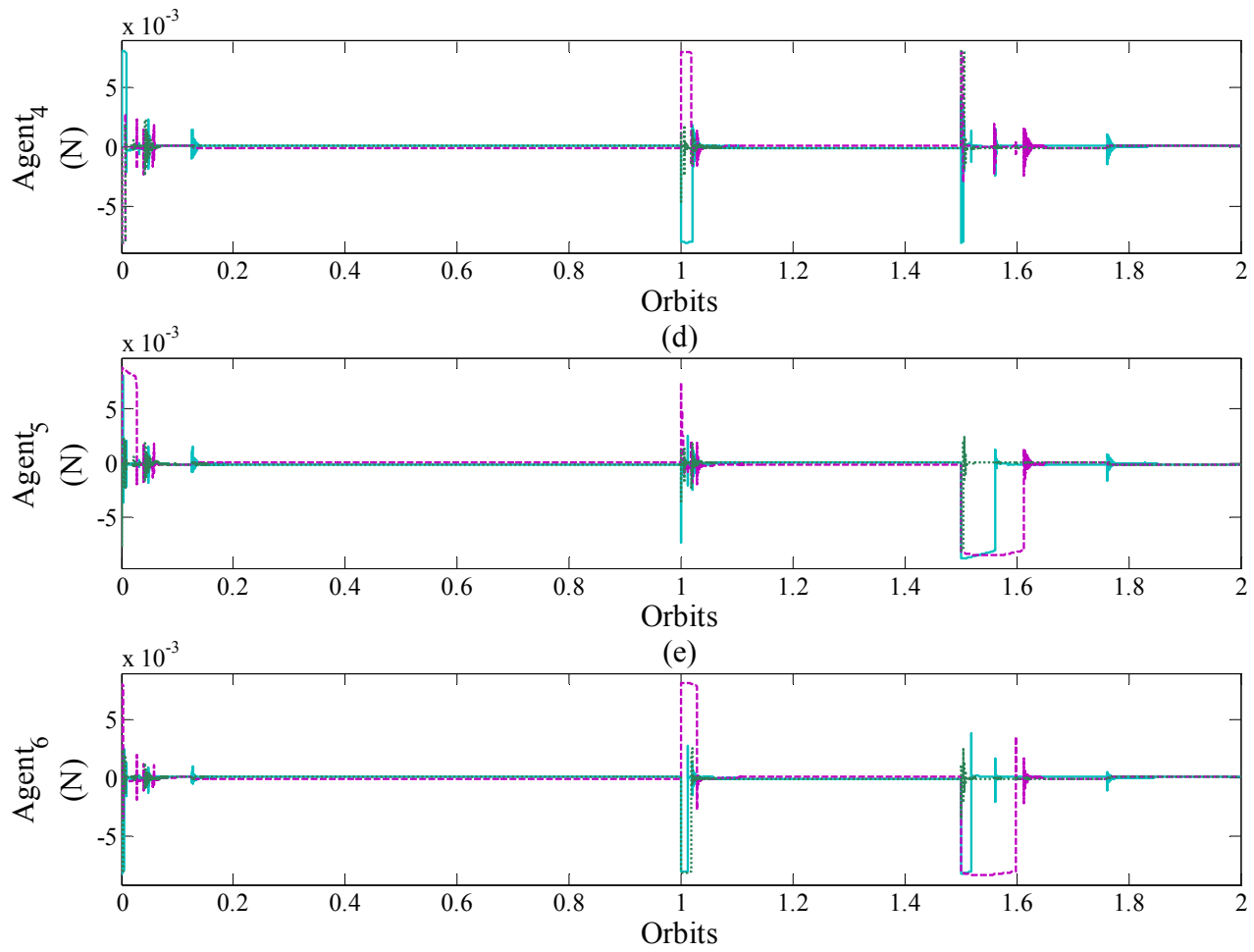

(f)

Figure 5.37: Estimated errors, formation reconfiguration 
When the formation is reconfigured, some agents need to move longer distances to reach their new positions, more error might occur, i.e. agent 1, 5, and 6, see Figure 5.37.

Lastly, all three types of reconfiguration are introduced to the system all at once after the first orbit. The results show that the CNN adaptive robust control is capable of regulating the system to follow all desired states with the presence of disturbance.

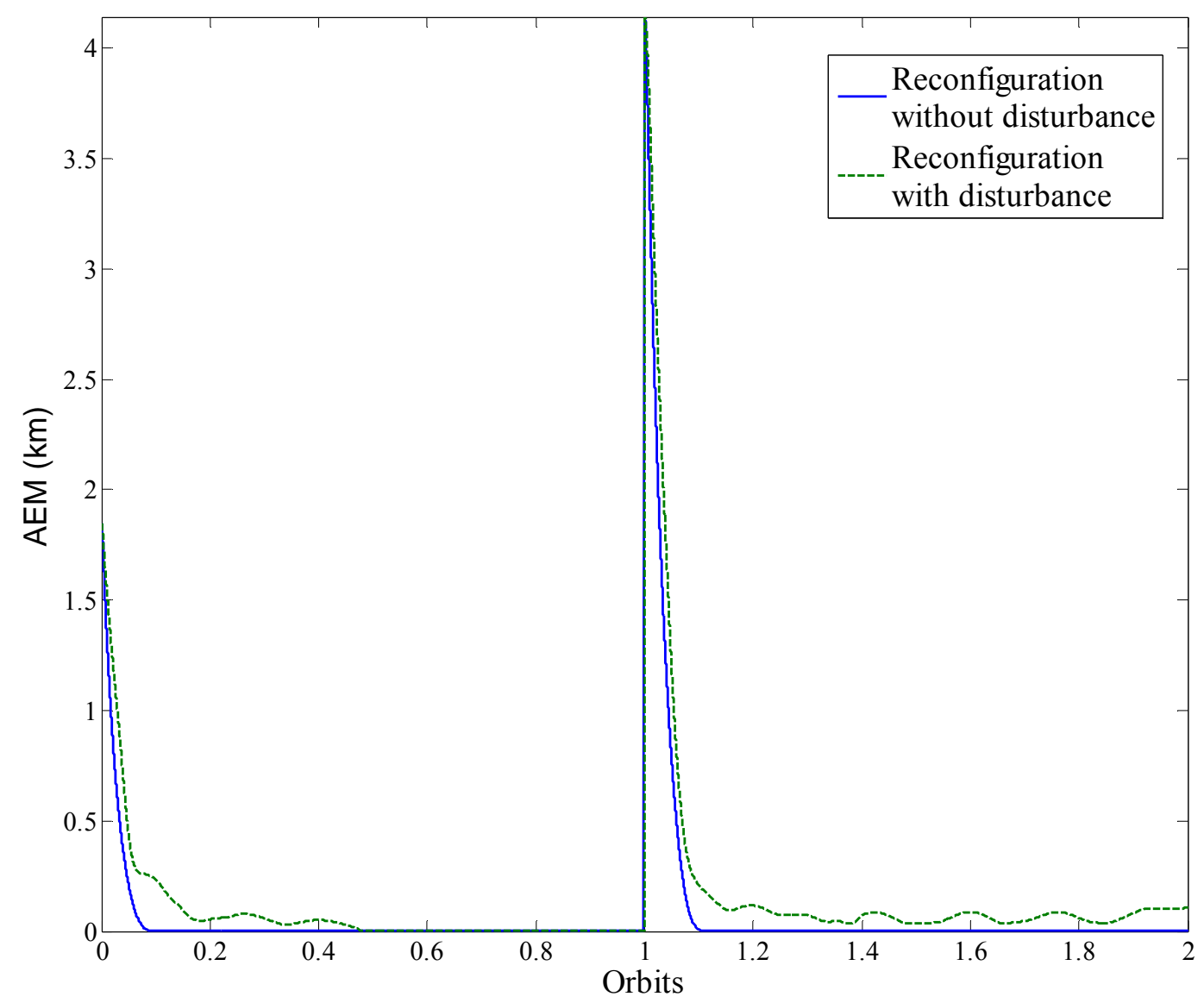

Figure 5.38: AEM, all reconfiguration

The AEM in Figure 5.38 shows that the proposed control law is not sensitive to external disturbance when Chebyshev Neural Networks is applied. It is reasonable that the system consumes more control effort when disturbance is present, Figure 5.39, and it is evident that actuator saturation was not reached. 


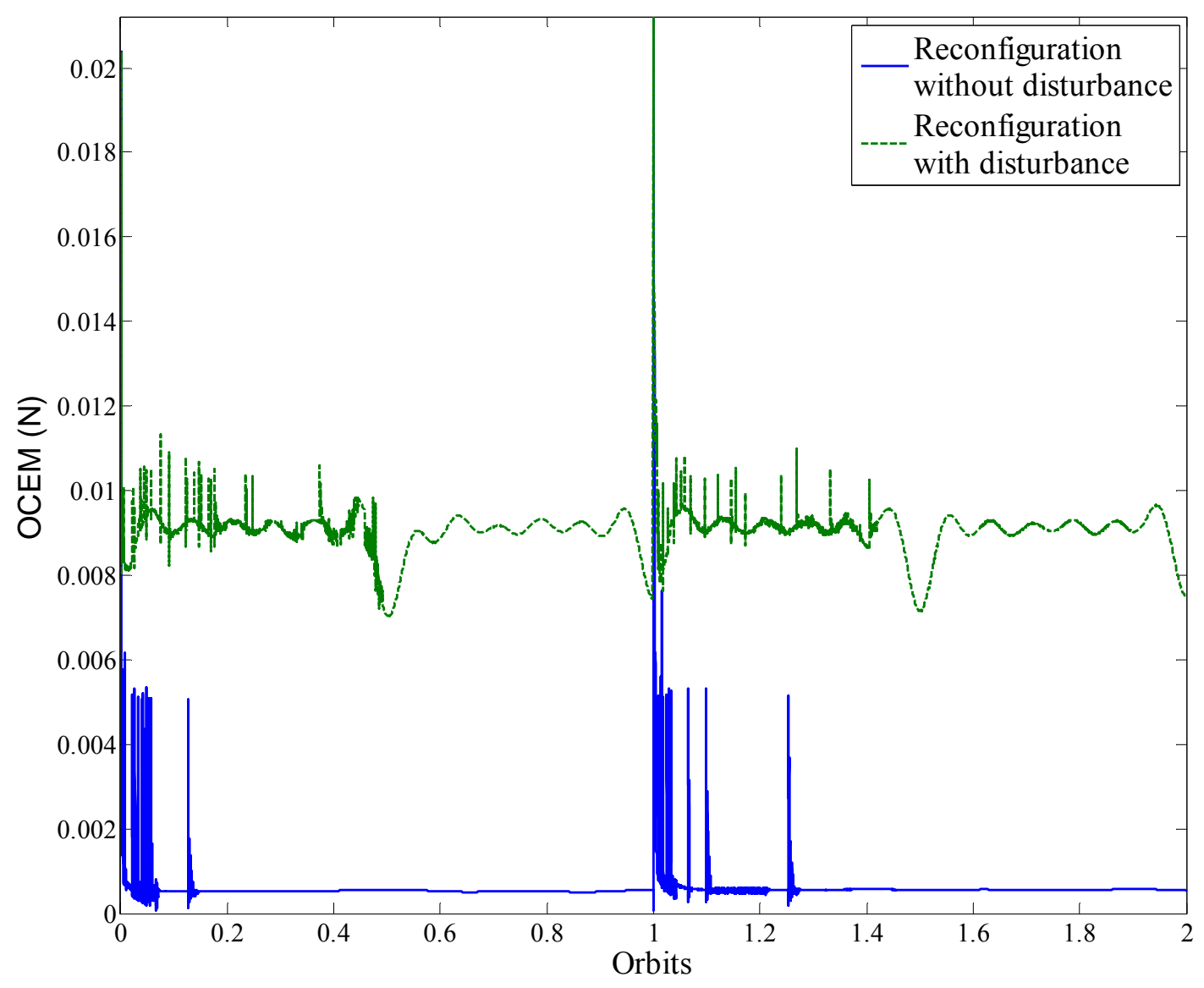

Figure 5.39: OCEM, all reconfiguration

The reconfiguration in reference trajectory or the shape of the formation did not degrade the response of the system, in fact with the use of this new adaptive control law, introducing uncertainty to the system is not an issue. Orbital reconfiguration brings practical contribution in space missions. With the use of reconfiguration, more flexible missions could be planned, and asteroids would be observed freely.

\subsection{CONCLUSIONS}

The Chebyshev polynomial functions chosen in this thesis estimate the system dynamics properly when the adaptive Chebyshev neural network is applied with the Terminal Sliding Mode Control to the multi-agent system. The position and velocity states errors are given to the Chebshev neural network, then the estimated dynamic are inputted to the control system. In order to increase the accuracy of the Chebyshev estimated value, large state errors are filtered out before feeding 
into the neural network. The results show that the proposed control law, where combining the adaptive control, robust control and neural network has given a great improvement in controlling the multi-agent system. In addition, the tracking errors converge without any adjustment in the control parameters when both actuator faults and sensor faults are simulated with the presence of external disturbances. Moreover, the adaptive ability in the proposed control allows the system to remain in homeostasis when the mass of the spacecraft increase. Hence, mass is no longer a major parameter that influences the controllability of the system. Furthermore, with the use of the neural network, the equation of motion that described the dynamic of the spacecraft is no longer needed in order to achieve precise control. More uncertainty can be handled by the control system on the assumption that it is within the capacity of the actuator system. Lastly, the ability to perform orbital reconfiguration, which included the reconfiguration in spacecraft formation and orbital trajectory, is a good example to illustrate the advancement in the proposed control law. With the use of the adaptive $\mathrm{CNN}$ and robust control, spacecraft formation and reference trajectory can be modified during flight. Keep in mind that when the reference trajectory changes, the minimum system control input required also changes. This new control law has brought a great deal of flexibility to a space mission where orbit reconfiguration in necessary. 


\section{CONCLUSIONS}

Consensus tracking states that a network of agents comes to agreement in regards to their final goal through communication among local agents or subsets of agents. To generalize the consensus tracking capabilities, consider thousands of spacecraft flying simultaneously wherein numerous subsets exist, each with their own designated tasks. By applying the proposed control law, different tasks assigned in the mission can be completed successfully while ensuring all agents work together as a team and communicate information among one another to avoid collisions and account for uncertain system dynamics. In this chapter, we review the contributions of the multiagent control techniques developed in this thesis. We breakdown the contributions based on the techniques presented in the previous chapters to outline possible future research in applying multiple spacecraft orbiting around asteroid.

\subsection{SUMMARY OF CONTRIBUTIONS}

Multiple agent consensus formation tracking demand advanced and reliable control systems to ensure fast, precise, and effective responses to different orbiting and formation reconfiguration commands. It is of great benefit to apply this research to novel asteroid exploration space missions. To facilitate proficiencies that would never be accomplished by monolithic large spacecraft, NASA and other organizations, such as the European Space Agency, have proposed several missions and mission statements to employ formation flying as a solution to reduce the costs and improve the flexibility of deep space asteroid exploration programs. Developing the technology for decentralized formation flying is remarkably complex and extraordinary. The objective of the space program initiated by the Space Systems Dynamics and Control (SSDC) laboratory at Ryerson University is to design a multi-agent consensus tracking control law that enhances the on board orbital control system on each agent of the consensus system to ensure that these challenging decentralized formation flying missions will be completed successfully. 


\subsubsection{Orbiting around Asteroid using Multiple Spacecraft}

Orbits around a small celestial body are considerably different from the Keplerian orbits in the two-body problem. Two factors that illustrate the differences between the problems are the irregular shape of an asteroid and the rotation of the asteroid. These coupling effects can lead to large energy and angular momentum changes within short time periods during the orbit of a spacecraft. It is rare that these effects happen to spacecraft motion around planetary bodies. The study of spacecraft motion about small astronomical bodies is new and challenging, especially because of these effects. When studying the orbital motion of an asteroid, the most significant features are the perturbation terms from the asteroid gravity field. The $2^{\text {nd }}$ degree and $2^{\text {nd }}$ order gravity coefficients, $C_{20}$ and $C_{22}$, respectively, were used in our investigation.

An investigation into the dynamics of specific asteroids, Gaspra, Ida, Vesta and Castalia, has been conducted wherein said asteroids are irregularly shaped and slowly rotating about their centre of mass. There is little existing literature that fully captures and simulates the effects of environmental forces on the system dynamics around asteroids. In contrast, the work presented in this thesis expands in such areas by examining environmental disturbances, namely solar radiation pressure force, solar gravitational force, and variance in the moment of inertia of the asteroid. Furthermore, the size-shape stability of the system is examined and the natural response and minimum required control input for a specific trajectory are presented and used as a baseline comparison.

\subsubsection{Decentralized Formation Flying}

Centralized relative motion control of spacecraft formations using thrusters is a well understood and an extensively studied problem. Despite recent advances, very few researchers have addressed the prospects of using decentralized formation flying to illustrate formation acquisition and reconfiguration maneuvers which is a major focus in this thesis. Insufficient effort has been made in the application of orbiting spacecraft around asteroids. The controllers available in the literature are based on linearized relative motion dynamics and only work in a small neighborhood of the origin.

To successfully exhibit coordinate cooperation, a suitable communication network and algorithm is of top priority in coordinated control. As such, three important issues must be addressed; consensus seeking, which commonly banks on the relationship between the graph 
topologies and the algebraic graph matrices, formation keeping of both centralized and decentralized approaches, and lastly, trajectory tracking. These issues have brought new interest to researchers because of their nonholonomic constraints on the dynamic systems. Consensus algorithms are developed to distribute tasks to multi-agents and only neighbour-to-neighbour communication between agents is provided; the information states of each agent are updated by the information states of their neighbour. From a control perspective, the fundamental objective of a consensus algorithm is to execute similar commands on the states of each agent in the network.

Decentralized formation flying around asteroids has not been fully examined in existing literature. This chapter addresses this notion by combining a nonlinear controller with consensus tracking. To further define the consensus as presented in this chapter, it should be mentioned that no leader is required and communication is linked only between necessary agents. This is done to reduce network complexity and the number of signal transmissions. The results obtained in this chapter verify the success of the application of multi-agents to perform missions around asteroids.

\subsubsection{Nonlinear CNN Adaptive Control}

In Chapter 3, a nonlinear control law is first developed to accomplish the consensus control orbiting around an asteroid. It is evident that the developed control law is able to follow the desired trajectory with the ability to maintain formation in the event of mission faults. In Chapter 4 , a robust control law is developed to take into account the larger magnitude of the disturbance and is subjected to a fault based mission. Lastly, in Chapter 5 a combination of the Chebyshev neural network with adaptive control is applied along with the robust control developed in Chapter 4 to tolerate the worst case fault that would be detrimental to the proposed space mission.

The major contribution of this work is the development and analysis of a distributed robust adaptive consensus formation tracking controller for second-order multi-agent systems using terminal sliding mode and CNN. In the proposed control scheme, the terminal sliding manifold is constructed based on lumped state errors that include absolute and relative state errors, and CNNs are employed to approximate unknown desired nonlinear functions in the dynamics of each agent. The dynamics of the plant are not given to the control system. Meanwhile, the smooth projection algorithm is applied to guarantee that the estimated parameters remain within some known bounded sets. Furthermore, the robust controller using the hyperbolic tangent function is employed to counteract $\mathrm{CNN}$ approximation errors and bounded external disturbances, which makes the 
proposed controller continuous, and therefore, keeps chattering to a minimum. Most importantly, the finite-time stability in both the reaching phase and the sliding phase is guaranteed by the Lyapunov theorem. Moreover, the proposed controller can force a group of agents to a desired time-varying trajectory even in the presence of unknown agents' dynamics and external disturbances as demonstrated in the simulations. Therefore, the proposed controller is robust against not only structured uncertainties, but also against those that are unstructured. Finally, comparative studies between the proposed controller and previously developed control law have shown that the performance of the new controller is superior to that of those proposed in previous chapters.

\subsubsection{Actuators/Sensors Faults and Formation Reconfiguration}

Since spacecraft formation flying has become an important field of research in the space industry due to cost benefits and mass savings that arise from this mode of operation, the development and implementation of robust and reliable control law for controlling the formation is necessary to ensure that the advantages of formation flying are effectively exploited. Autonomous coordinated control, precise formation-keeping, and reconfiguration of formation geometry in the presence of actuator faults are areas critical to the success of any proposed mission involving spacecraft flying in formation.

\subsubsection{Variations of Spacecraft Mass}

The mass of the spacecraft is changed from time to time in the design process. Moreover, the mass of the spacecraft will decrease during the mission when propellant is used. Therefore, a good control law is one which works independently of the mass of the spacecraft. Adaptive control will allow the control law to be able to control with consideration of unknown parameters without control reconfiguration.

To achieve reliable autonomous spacecraft systems, an adaptive control must be implemented. The terminal sliding manifold based on lumped state errors for the consensus tracking control of second-order multi-agent systems is proposed. Furthermore, the controller is non-singular because there exists no negative fractional powers. In comparison with the terminal sliding mode-based consensus tracking controller, control signals do not need to be exchanged between neighboring agents, which not only reduces the information flow but also avoids the algebraic loop problem existing in the controller. A Chebyshev polynomial is used to estimate the 
state dynamics and is fed into the terminal sliding mode controller. By applying the Chebyshev Neural Networks to different aspects of the model, the controller can account for more parameter uncertainties to advance the autonomous capabilities of the system.

\subsection{FUTURE WORKS}

\subsubsection{Plant Model Improvement}

The second degree and second order gravity potential constant is used in this thesis. In order to more accurately model the dynamics of an asteroid, higher order models can be used. A time varying disturbance model was used to replicate the environmental disturbance; the disturbances considered in this thesis have a larger magnitude than those found in the natural environment. Therefore, if a solar radiation pressure force, solar gravitational force, and/or variance in the moment of inertia of the asteroid is included in the plant, the results will more closely resemble those of a real mission. More control effort will be relaxed from this over projection and this effort could be relocated to other areas. Different asteroids are investigated and the proposed control law performs well with the examined situation. However, if a more suitable reference trajectory were to be examined with each asteroid, the results may have greater improvement but less state error and control effort would be required.

\subsubsection{Simulation Model Improvement}

Sensor faults and actuator faults have been examined. The CNN adaptive robust control proved that it is capable of handling both faults with the presence of disturbance. However, future research can improve the results obtained by filtering the sensor faults through the use of an observer before feeding the information into the control system. In this thesis, thrusters provide the primary actuation force for the spacecraft. In the previously existing literature, there are few asteroid exploration missions that use Solar Radiation Pressure (SRP) for actuation. Since asteroids are much smaller than celestial bodies, such as planets, a higher solar radiation pressure gradient may be available when considering a spacecraft orbiting the shadow region of a planet as there may be no solar exposure. If an SRP actuator model is applied to the system, it will add significant value to the research. When the location of the solar sail is orientated properly toward the Sun, it will provide unlimited and continuous power to propel the spacecraft. In a couple of the examined scenarios, although large numerical tracking errors exist while the system is undergoing actuator 
saturation, the proposed control law is capable of reconfiguring the agents back to their desired states. This is a direct result of the assumption made wherein there is an unlimited supply of propellant carried onboard. Therefore, if an optimized fuel consumption model can be modelled into the system, an increase in the credibility of this research for a space mission will be achieved.

\subsubsection{Attitude and Orbit Control System}

Although the proposed control law in this study is for formation tracking, it serves as a good foundation toward attitude control. A recent study has stated that there are strong coupling effects between attitude control and orbital dynamics. The control of pose, attitude, and tracking of spacecraft, simultaneously, are gaining more interest instead of the attitude alone. Future work should include coupling effects of the aforementioned as they have not been studied under an asteroid model.

Aside from studying the reference trajectory optimization and the coupling effects for space dynamic systems, an improvement in the adaptive control will be great future work. Adaptive control is one of the best contributions toward the current study, however, it involves high level of computation power, and many gains are introduced in the control law. The more modelling that is required suggests more aspects to be considered. This in turn evolves to a greater number of control and estimation parameters that need to be considered. Recently, with the increase in computational power, optimization using a genetic algorithm is regaining its popularity, which is one of the ideal ways to further refine or combine with adaptive control. Also, orbital and formation reconfiguration have been achieved in the present thesis, and it will also be of great benefit to the space research if the consensus network is reconfigured. Therefore, multi-agent and multi systems can be achieved. 


\subsection{CONCLUDING REMARKS}

Various uncertainties exist that may be detrimental to the success of the mission and cause the failure of a spacecraft orbiting around an asteroid. This is true in the case when dealing with monolithic satellites; if any part of the spacecraft fails, the entire mission may be jeopardized. To avoid this situation, introducing redundancies through decentralized multi-agents system would ensure that even in a scenario where one agent is unresponsive and/or damaged, the remaining agents can carry out their tasks and still lead to a mission's success. This notion would increase the flexibility of the system while maintaining a safety factor in case of any failures. Successful implementation of the proposed methodology will greatly enhance the reliability of the spacecraft, while allowing for potentially significant overall mission cost reduction. 


\section{BIBLIOGRAPHY}

1 Hall, L., "Welcome to the Planets". Jet Propulsion Laboratory of the California Institute of Technology, 2005.

2 Chamberlin, A. B., "Solar System Dynamics: How Many Solar System Bodies", NASA Jet Propulsion Laboratory, URL: http://ssd.jpl.nasa.gov/?body_count [cited 24 May 2013]

${ }^{3}$ Hudson, R. S., and Ostro, S. J., "Shape of Asteroid 4769 Castalia (1989 Pb) from Inversion of Radar Images”, Science, Vol. 263, No. 5149, 1994, pp. 940-943.

${ }^{4}$ Hudson, R. S., and Ostro, S. J., "Shape and Non-Principal Axis Spin State of Asteroid 4179 Toutatis", Science-New York Then Washington-, 1995, pp. 84-84.

5 Scheeres, D., Ostro, S., Hudson, R., and Werner, R., "Orbits Close to Asteroid 4769 Castalia", Icarus, Vol. 121, No. 1, 1996, pp. 67-87.

${ }^{6}$ Farinella, P., Paolicchi, P., and Zappala, V., “Analysis of the Spin Rate Distribution of Asteroids”, Astronomy and Astrophysics, Vol. 104, 1981, pp. 159-165.

$7 \mathrm{Hu}, \mathrm{W}$., "Orbital Motion in Uniformly Rotating Second Degree and Order Gravity Fields", Aerospace Engineering. Vol. Doctor of Philosophy, The University of Michigan, ProQuest Dissertations and Theses, 2002, p. 153.

${ }^{8} \mathrm{Hu}$, W., and Scheeres, D. J., "Spacecraft Motion About Slowly Rotating Asteroids", Journal of guidance, control, and dynamics, Vol. 25, No. 4, 2002, pp. 765-775.

9 Scheeres, D. J., "Dynamics About Uniformly Rotating Triaxial Ellipsoids: Applications to Asteroids", Icarus, Vol. 110, No. 2, 1994, pp. 225-238.

${ }^{10}$ Schwehm, G., "Rosetta/Cnsr, Esa's Planetary Cornerstone Mission”, Physics and Mechanics of Cometary Materials, Vol. 302, 1989, pp. 11-15.

${ }^{11}$ Yang, C., He-xi, B., and Jun-feng, L., "Trajectory Analysis and Design for a Jupiter Exploration Mission”, Chinese Astronomy and Astrophysics, Vol. 37, No. 1, 2013, pp. 77-89.

12 Kapila, V., Sparks, A. G., Buffington, J. M., and Yan, Q., "Spacecraft Formation Flying: Dynamics and Control", Journal of Guidance, Control, and Dynamics, Vol. 23, No. 3, 2000, pp. 561-564.

13 Sabol, C., Burns, R., and McLaughlin, C. A., "Satellite Formation Flying Design and Evolution”, Journal of Spacecraft and Rockets, Vol. 38, No. 2, 2001, pp. 270-278. 
14 Godard, G., and Kumar, K. D., "Fault Tolerant Reconfigurable Satellite Formations Using Adaptive Variable Structure Techniques", Journal of guidance, control, and dynamics, Vol. 33, No. 3, 2010, pp. 969-984.

15 Varma, S., and Kumar, K. D., "Multiple Satellite Formation Flying Using Differential Aerodynamic Drag”, Journal of Spacecraft and Rockets, Vol. 49, No. 2, 2012, pp. 325-336.

16 Conkey, D. E., Dell, G. T., Good, S. M., and Bristow, J., "Eo-1 Formation Flying Using Autocon”, Aerospace Conference Proceedings, 2000 IEEE, Vol. 7, 2000, pp. 55-61.

${ }^{17}$ Stephens, G. L., Vane, D. G., Tanelli, S., Im, E., Durden, S., Rokey, M., Reinke, D., Partain, P., Mace, G. G., and Austin, R., "Cloudsat Mission: Performance and Early Science after the First Year of Operation”, Journal of Geophysical Research: Atmospheres (1984-2012), Vol. 113, No. D8, 2008,

${ }^{18}$ Mace, G. G., Zhang, Q., Vaughan, M., Marchand, R., Stephens, G., Trepte, C., and Winker, D., "A Description of Hydrometeor Layer Occurrence Statistics Derived from the First Year of Merged Cloudsat and Calipso Data", Journal of Geophysical Research: Atmospheres (1984-2012), Vol. 114, No. D8, 2009,

${ }^{19}$ Giglio, L., Csiszar, I., and Justice, C. O., "Global Distribution and Seasonality of Active Fires as Observed with the Terra and Aqua Moderate Resolution Imaging Spectroradiometer (Modis) Sensors", Journal of Geophysical Research: Biogeosciences, Vol. 111, No. G2, 2006, pp. G02016 1-12.

20 Parkinson, C. L., "Aqua: An Earth-Observing Satellite Mission to Examine Water and Other Climate Variables", Geoscience and Remote Sensing, IEEE Transactions on, Vol. 41, No. 2, 2003, pp. 173-183.

${ }^{21}$ Savtchenko, A., Ouzounov, D., Ahmad, S., Acker, J., Leptoukh, G., Koziana, J., and Nickless, D., "Terra and Aqua Modis Products Available from Nasa Ges Daac", Advances in Space Research, Vol. 34, No. 4, 2004, pp. 710-714.

${ }^{22}$ Clemente, D. C., and Atkins, E. M., “Optimization of a Tetrahedral Satellite Formation”, Journal of spacecraft and rockets, Vol. 42, No. 4, 2005, pp. 699-710.

${ }^{23}$ Guzman, J., and Schiff, C., "A Preliminary Study for a Tetrahedron Formation: Quality Factors and Visualization”, AIAA Guid, Nav, \& Contr, Conf, 2002,

${ }^{24}$ Guzman, J. J., "Tetrahedron Formation Control”, Journal of the Astronautical Sciences, Vol. 51, No. 4, 2003, pp. 419-432.

${ }^{25}$ Belanger, G. M., Ananyev, S., Speyer, J. L., Chichka, D. F., and Carpenter, J. R., "Decentralized Control of Satellite Clusters under Limited Communication”, Journal of guidance, control, and dynamics, Vol. 29, No. 1, 2006, pp. 134-145. 
26 Carpenter, J. R., “A Preliminary Investigation of Decentralized Control for Satellite Formations", Aerospace Conference Proceedings, 2000 IEEE, Vol. 7, 2000, pp. 63-74 vol.7.

27 Chang, I., Park, S.-Y., and Choi, K.-H., "Decentralized Coordinated Attitude Control for Satellite Formation Flying Via the State-Dependent Riccati Equation Technique", International Journal of Non-Linear Mechanics, Vol. 44, No. 8, 2009, pp. 891-904.

${ }^{28}$ Lafferriere, G., Williams, A., Caughman, J., and Veerman, J., "Decentralized Control of Vehicle Formations", Systems \& control letters, Vol. 54, No. 9, 2005, pp. 899-910.

${ }^{29}$ Massioni, P., Keviczky, T., and Verhaegen, M., "New Approaches to Distributed Control of Satellite Formation Flying", Proc. 3rd International Symposium on Formation Flying, Missions and Technologies, 2008,

${ }^{30}$ Ren, W., and Beard, R., "Decentralized Scheme for Spacecraft Formation Flying Via the Virtual Structure Approach”, Journal of Guidance, Control, and Dynamics, Vol. 27, No. 1, 2004, pp. 73-82.

${ }^{31}$ Russell Carpenter, J., "Decentralized Control of Satellite Formations”, International Journal of Robust and Nonlinear Control, Vol. 12, No. 2 - 3, 2002, pp. 141-161.

32 VanDyke, M. C., and Hall, C. D., "Decentralized Coordinated Attitude Control within a Formation of Spacecraft", Journal of Guidance, Control, and Dynamics, Vol. 29, No. 5, 2006, pp. 1101-1109.

${ }^{33}$ Husheng, L., Lifeng, L., and Poor, H. V., "Multicast Routing for Decentralized Control of Cyber Physical Systems with an Application in Smart Grid", Selected Areas in Communications, IEEE Journal on, Vol. 30, No. 6, 2012, pp. 1097-1107.

34 Mazo, M., and Tabuada, P., "Decentralized Event-Triggered Control over Wireless Sensor/Actuator Networks", Automatic Control, IEEE Transactions on, Vol. 56, No. 10, 2011, pp. 2456-2461.

35 Shahraeini, M., Javidi, M. H., and Ghazizadeh, M. S., "Comparison between Communication Infrastructures of Centralized and Decentralized Wide Area Measurement Systems", Smart Grid, IEEE Transactions on, Vol. 2, No. 1, 2011, pp. 206-211.

36 Stanković, S. S., Stanković, M. S., and Stipanović, D. M., "Consensus Based Overlapping Decentralized Estimation with Missing Observations and Communication Faults", Automatica, Vol. 45, No. 6, 2009, pp. 1397-1406.

37 Yang, P., Freeman, R. A., Gordon, G. J., Lynch, K. M., Srinivasa, S. S., and Sukthankar, R., "Decentralized Estimation and Control of Graph Connectivity for Mobile Sensor Networks", Automatica, Vol. 46, No. 2, 2010, pp. 390-396. 
38 Joordens, M. A., and Jamshidi, M., "Consensus Control for a System of Underwater Swarm Robots”, Systems Journal, IEEE, Vol. 4, No. 1, 2010, pp. 65-73.

${ }^{39}$ Bonabeau, E., Theraulaz, G., Deneubourg, J.-L., Aron, S., and Camazine, S., "Self-Organization in Social Insects", Trends in Ecology \& Evolution, Vol. 12, No. 5, 1997, pp. 188-193.

40 Curtis, S., Rilee, M., Clark, P., and Marr, G., "Use of Swarm Intelligence in Spacecraft Constellations for the Resource Exploration of the Asteroid Belt", Proceedings of the Third International Workshop on Satellite Constellations and Formation Flying, 2003, pp. 2426.

${ }^{41}$ Truszkowski, W., Rash, J., Rouff, C., and Hinchey, M., "Asteroid Exploration with Autonomic Systems", Engineering of Computer-Based Systems, 2004. Proceedings. 11th IEEE International Conference and Workshop on the, 2004, pp. 484-489.

42 Rouff, C., Hinchey, M., Rash, J., and Truszkwoski, W., "Systems of Systems Verification", Infotech@Aerospace. American Institute of Aeronautics and Astronautics, 2005.

${ }^{43}$ Ren, W., and Beard, R. W., Distributed Consensus in Multi-Vehicle Cooperative Control: Theory and Applications: Springerverlag London Limited, 2008.

${ }^{44}$ Ren, W., Beard, R. W., and Atkins, E. M., "Information Consensus in Multivehicle Cooperative Control”, Control Systems, IEEE, Vol. 27, No. 2, 2007, pp. 71-82.

${ }^{45}$ Suiyang, K., Lihua, X., and Zhihong, M., "Robust Finite-Time Consensus Tracking Algorithm for Multirobot Systems", Mechatronics, IEEE/ASME Transactions on, Vol. 14, No. 2, 2009, pp. 219-228.

${ }^{46}$ Leshno, M., Lin, V. Y., Pinkus, A., and Schocken, S., "Multilayer Feedforward Networks with a Nonpolynomial Activation Function Can Approximate Any Function", Neural Networks, Vol. 6, No. 6, 1993, pp. 861-867.

${ }^{47}$ Sanner, R. M., and Slotine, J. J. E., "Gaussian Networks for Direct Adaptive Control”, Neural Networks, IEEE Transactions on, Vol. 3, No. 6, 1992, pp. 837-863.

48 Tsu-Tian, L., and Jin-Tsong, J., "The Chebyshev-Polynomials-Based Unified Model Neural Networks for Function Approximation", Systems, Man, and Cybernetics, Part B: Cybernetics, IEEE Transactions on, Vol. 28, No. 6, 1998, pp. 925-935.

49 Zou, A.-M., and Kumar, K. D., "Adaptive Attitude Control of Spacecraft without Velocity Measurements Using Chebyshev Neural Network", Acta Astronautica, Vol. 66, No. 5-6, 2010, pp. 769-779. 
${ }^{50}$ Lee, Y., and Zak, S. H., "Uniformly Ultimately Bounded Fuzzy Adaptive Tracking Controllers for Uncertain Systems”, Fuzzy Systems, IEEE Transactions on, Vol. 12, No. 6, 2004, pp. 797-811.

51 Cheng, L., Hou, Z.-G., Tan, M., Lin, Y., and Zhang, W., "Neural-Network-Based Adaptive Leader-Following Control for Multiagent Systems with Uncertainties", Neural Networks, IEEE Transactions on, Vol. 21, No. 8, 2010, pp. 1351-1358.

52 Zeng-Guang, H., Long, C., and Min, T., "Decentralized Robust Adaptive Control for the Multiagent System Consensus Problem Using Neural Networks", Systems, Man, and Cybernetics, Part B: Cybernetics, IEEE Transactions on, Vol. 39, No. 3, 2009, pp. 636647.

${ }^{53}$ Das, A., and Lewis, F. L., "Cooperative Adaptive Control for Synchronization of Second-Order Systems with Unknown Nonlinearities", International Journal of Robust and Nonlinear Control, Vol. 21, No. 13, 2011, pp. 1509-1524.

54 Gang, C., and Lewis, F. L., "Distributed Adaptive Tracking Control for Synchronization of Unknown Networked Lagrangian Systems", Systems, Man, and Cybernetics, Part B: Cybernetics, IEEE Transactions on, Vol. 41, No. 3, 2011, pp. 805-816.

55 Zou, A. M., Kumar, K. D., and Hou, Z. G., “Distributed Consensus Control for Multi - Agent Systems Using Terminal Sliding Mode and Chebyshev Neural Networks” , International Journal of Robust and Nonlinear Control, Vol. 23, No. 3, 2013, pp. 334-357.

56 Nhan, N., John, B., and Abraham, I., "Least-Squares Adaptive Control Using Chebyshev Orthogonal Polynomials”, Infotech@Aerospace 2011. American Institute of Aeronautics and Astronautics, 2011, pp. 1-22.

57 Wei, R., "Distributed Cooperative Attitude Synchronization and Tracking for Multiple Rigid Bodies”, Control Systems Technology, IEEE Transactions on, Vol. 18, No. 2, 2010, pp. 383392.

58 Ziyang, M., Wei, R., Cao, Y., and You, Z., "Leaderless and Leader-Following Consensus with Communication and Input Delays under a Directed Network Topology", Systems, Man, and Cybernetics, Part B: Cybernetics, IEEE Transactions on, Vol. 41, No. 1, 2011, pp. 7588.

59 Chopra, N., and Spong, M., "Output Synchronization of Nonlinear Systems with Relative Degree One", Recent Advances in Learning and Control. Vol. 371, Springer London, 2008, pp. 51-64.

${ }^{60}$ Hill, D. J., and Jun, Z., "Global Synchronization of Complex Dynamical Networks with NonIdentical Nodes", Decision and Control, 2008. CDC 2008. 47th IEEE Conference on, 2008, pp. 817-822. 
${ }^{61}$ Hongkeun, K., Hyungbo, S., and Jin Heon, S., "Output Consensus of Heterogeneous Uncertain Linear Multi-Agent Systems”, Automatic Control, IEEE Transactions on, Vol. 56, No. 1, 2011, pp. 200-206.

${ }^{62}$ Kumar, K. D., Fundamentals of Dynamics and Control of Space Systems, Toronto, Canada: Ryerson University, 2006.

${ }^{63}$ Curtis, H., "Sphere of Influence", Orbital Mechanics for Engineering Students. $6^{\text {th }}$ ed., Elsevier, Amsterdam, 2008, pp. 354-359.

64 Godard, G., "Fault Tolerant Control of Spacecraft", Aerospace Engineering. Vol. Doctor of Philosophy, Ryerson University, Ryerson University, 2010, p. 297.

65 Scheeres, D. J., Ostro, S. J., Hudson, R., and Werner, R. A., “Orbits Close to Asteroid 4769 Castalia", Icarus, Vol. 121, No. 1, 1996, pp. 67-87.

66 Scheeres, D., "Orbit Mechanics About Small Asteroids", 20th International Symposium on Space Flight Dynamics, 2007,

67 Scheeres, D., "Orbit Mechanics About Asteroids and Comets”, Journal of Guidance, Control and Dynamics, Vol. 35, No. 3, 2012, pp. 987-997.

${ }^{68}$ Ren, W., "Consensus Seeking, Formation Keeping, and Trajectory Tracking in Multiple Vechicle Cooperative Control”. Vol. Doctor of Philosophy, Brigham Young University, 2004, p. 159. 